\title{
Tuning of the Relative Phases of a Rotary Travelling Wave Oscillator
}

\author{
by \\ Raleigh Smith, B.Sc. Eng., P. Eng.

\begin{abstract}
A thesis submitted to the
Faculty of Graduate and Postdoctoral Affairs

in partial fulfillment of the requirements for the degree of
\end{abstract}

Master of Applied Science in Electrical and Computer Engineering

Ottawa-Carleton Institute for Electrical and Computer Engineering

Department of Electronics Engineering

Carleton University

Ottawa, Ontario

December, 2014

(C) Copyright

Raleigh Smith, 2014 


\section{Abstract}

The work that follows describes the implementation and use of a simple phase detector for phase measurement, and an algorithm to adjust variable Transmission Line (TL) capacitance to tune the phases of an existing RTWO.

Symmetrical XOR (SXOR) phase detectors were designed, fabricated in SiGe BiCMOS and tested to resolve $1^{\circ}$ of phase error over $45^{\circ} \pm 10^{\circ}$ and $90^{\circ} \pm 10^{\circ}$ up to $12 \mathrm{GHz}$. These phase detectors were used to measure and calibrate the phase of $45^{\circ}$ transmission line sections of an $18-\mathrm{GHz}$ RTWO fabricated in SiGe BiCMOS. This phase tuning was carried out by a simple phase alignment algorithm that can be implemented easily in verilog. This RTWO phase tuning system was shown to function correctly in simulation. Although the fabricated version did not converge correctly, the algorithm was able to adjust the phase of RTWO TL sections. The circuit problem preventing convergence is understood and can be corrected. 
To Dolce and Dessi. 


\section{Acknowledgments}

I sincerely thank my supervisor John Rogers and co-supervisor Calvin Plett for accepting me as a student, helping me choose this topic, and providing me with technical guidance and direction from the beginning to the end of this thesis. Also, I am very grateful to Kimia Taghizadeh Ansari for helping me with the NXP QUBIC kit setup, the work she did including multiple copies of my phase detector and buffer in her RTWO tape out and for giving some of these die to test. Also special thanks is owed to Dr. Patrice Gamand of NXP for providing us access their QUBIC SiGe BiCMOS process, the NXP staff for fabricating our designs and providing us with finished die, and in particular to Dr. Olivier Tesson of NXP for handling the fabrication details and answering many technical questions.

I also must thank Scott Bruce for his ongoing Linux support; Dave Kirky of Cadence Design systems for his continued support - especially in helping me to get AMS Designer working; Rob Vandusen of Carleton University and John Quan of Skyworks Solutions, Inc. for all the chip-on-board wire bonding; CMC for their tool support and lending us their high speed oscilloscope; and Blazenka Power for her persistence with a certain cable supplier.

Additionally, I am very grateful for the generous help given to me by many students in the Department of Electronics at Carleton University. Specifically, my friend 
and colleague Augusto Lima for convincing me to take this degree, for his encouragement and all the technical and non-technical discussions. Also, Zhanjun (JJ) Bai and Xing Zhou for all the tool and technical discussions, as well as many other graduate students for their help including Dan Lanfranconi, Stephen Toth, Tom Pepler, Tyler Ross, Che Knisely and Behzad Yadegari. 


\section{Table of Contents}

$\begin{array}{lll}\text { Abstract } & \text { ii }\end{array}$

Acknowledgments iv

Table of Contents vi

List of Tables $\quad x$

List of Figures $\quad$ xi

Nomenclature $\quad$ xv

1 Introduction 1

1.1 Motivation . . . . . . . . . . . . . . . . . . . 1

1.2 RTWO Phase Tuning Applications . . . . . . . . . . . . . 3

1.3 Thesis Objectives and Scope . . . . . . . . . . . . . 6

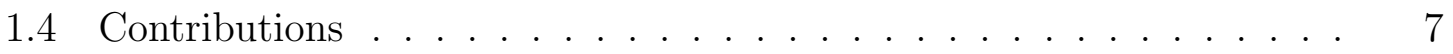

1.5 Thesis Organization $\ldots \ldots \ldots \ldots \ldots \ldots \ldots \ldots$

2 RTWO Concepts $\quad 9$

2.1 Oscillator Fundamentals $\ldots \ldots \ldots \ldots$

2.2 The Distributed Oscillator . . . . . . . . . . . . . . . . 11

2.3 RTWO Frequency Calculation . . . . . . . . . . . . . . . . 12 
2.4 Superposition and Injection Locking . . . . . . . . . . . . . . . 13

2.5 Phase Adjustment Concepts . . . . . . . . . . . . . . . . . . . . 17

2.6 Phase Tuning Limits . . . . . . . . . . . . . . . . . . . . . . . . . . . 18

2.7 Phase Change Calculation . . . . . . . . . . . . . . . . . . . . . . . . 19

3 RTWO Phase Calibration $\quad 22$

3.1 The System Concept . . . . . . . . . . . . . . . . . . . . 22

3.2 Proof of Concept - Matlab ${ }^{\circledR} /$ Simulink $^{\circledR}$. . . . . . . . . . . . . . . 23

3.2.1 RTWO Phase Measurement . . . . . . . . . . . . . 26

3.2.2 The Simulink ${ }^{\circledR}$ RTWO Block . . . . . . . . . . . . . . . . 30

3.2.3 The Phase Alignment Algorithm . . . . . . . . . . . . . . . 34

3.3 Proof of Concept - Cadence ${ }^{\circledR}$ Virtuoso ${ }^{\circledR}$ AMS Designer . . . . . . . . 37

3.3.1 RTWO Phase Measurement . . . . . . . . . . . . . . . . . 42

3.3.2 The Virtuoso ${ }^{\circledR}$ AMS Designer RTWO Block . . . . . . . . . . 45

3.3.3 The Phase Alignment Algorithm . . . . . . . . . . . . . . . . 48

4 Phase Measurement Circuit $\quad 50$

4.1 Phase Detector Selection . . . . . . . . . . . . . . . 50

4.2 CML Circuit Implementation _ . . . . . . . . . . . . . . . . . 52

4.3 Removal of Unequal Transit Delays . . . . . . . . . . . . . . . . . 54

4.4 Input Biasing and ECL Outputs . . . . . . . . . . . . . . . . . 59

4.5 SXOR Device Layout . . . . . . . . . . . . . . . . . 60

4.6 Phase Detector Rev1 versus Rev2 . . . . . . . . . . . . . . . 62

5 RTWO Phase Tuning Verification $\quad 64$

5.1 Extracted RTWO Phase Detector Verification . . . . . . . . . . 64

5.2 RTWO System Simulation Set-up . . . . . . . . . . . . . . . 72 
5.3 RTWO System Simulation - Typical Results . . . . . . . . . . 76

5.4 RTWO System Simulation - Updated from Fabrication . . . . . . 78

6 Experimental Results $\quad 85$

6.1 SXOR Phase Detector Test Set-up and Procedure . . . . . . . . 85

6.2 SXOR Phase Detector Test Set-up Calibration . . . . . . . . . . . 92

6.2.1 Direct Test Set-up Calibration . . . . . . . . . . . . . . . . 93

6.2.2 Indirect Test Set-up Calibration . . . . . . . . . . . . . . . . 94

6.3 SXOR Phase Detector Test Results . . . . . . . . . . . . . 97

6.4 Discussion of Results - SXOR Phase Detector . . . . . . . . . . . 104

6.5 RTWO Phase Alignment Test Set-up and Procedure . . . . . . . 107

6.6 RTWO Phase Alignment Test Results . . . . . . . . . . . . . . . . 113

6.7 Discussion of Results - RTWO Phase Alignment . . . . . . . . . . 117

7 Conclusions and Recommendations $\quad 120$

7.1 Summary of Work . . . . . . . . . . . . . . . 120

7.2 Contributions . . . . . . . . . . . . . . . . . . 122

7.3 Improvement and Future Work . . . . . . . . . . . . . . 122

$\begin{array}{ll}\text { List of References } & 124\end{array}$

Appendix A Additional Simulink RTWO Block Descriptions 128

A.1 Sub-Blocks of the RTWO $24 \mathrm{MHz}$ Block . . . . . . . . . 128

A.2 Gain Blocks . . . . . . . . . . . . . . . . . . . 132

Appendix B Phase Calibration Algorithm Code 134

B.1 Cadence Verilog Code . . . . . . . . . . . . . . . . . . . . . 134

B.2 FPGA Verilog Code . . . . . . . . . . . . . . . 138 
B.3 Matlab Code . . . . . . . . . . . . . . . . . . . . . . . . 151

Appendix C Test and Evaluation Printed Circuit Boards 157

C.1 Phase Detector PCB . . . . . . . . . . . . . . . . . . 157

C.2 RTWO Phase Alignment PCB . . . . . . . . . . . . . 160 


\section{List of Tables}

3.1 Transmission Line Phase Delay at Simulation Start . . . . . . . . 32

3.2 Transmission Line Phase Delay at Simulation End . . . . . . . . . . 33

3.3 Phase Alignment Simulaiton Results _. . . . . . . . . . . . 45

5.1 Monte Carlo Simulation Corner Definitions . . . . . . . . . . . 65

5.2 Summary of Monte Carlo Results for $45^{\circ} \pm 10^{\circ}$, Fast, Extracted ... 68

5.3 Summary of Monte Carlo Results for $45^{\circ} \pm 10^{\circ}$, Slow, Extracted . . 70

5.4 Summary of Monte Carlo Results for $90^{\circ} \pm 10^{\circ}$, Extracted $\ldots \ldots .72$

6.1 SXOR Test Card Voltage, Current and Power Dissipation . . . . . . . 92

6.2 Summary of Measured Results for $45^{\circ} \pm 10^{\circ}-12.0 \mathrm{GHz}$, Measured . . 102

6.3 Summary of Measured Results for $90^{\circ} \pm 10^{\circ}-12.0 \mathrm{GHz}$, Measured . . 103

6.4 RTWO Tuning Test Card Voltage, Current and Power Dissipation . . 112 


\section{List of Figures}

1.1 Idealized Illustration of a Four Section RTWO [1] . . . . . . . . . . 2

1.2 Fractional-N ADPLL with RTWO DCO [2] . . . . . . . . . . 4

1.3 RTWO DCO in a Digital Radio Polar Transmitter [3] . . . . . . . . 5

1.4 RTWO DCO in a 6-Port Network Receiver [4] . . . . . . . . . 5

2.1 Classical Linear Feedback Model . . . . . . . . . . . . . . . . . . . . 9

2.2 [5] a) TL Loop; b) Twist; and c) Differential RTWO TL Structure . 10

2.3 a) Conceptual RTWO [1]; b) Single Cross-Over; c) Three Cross-Overs 11

2.4 a) $\lambda / 4$ SWO; b) $\lambda / 2$ SWO [5]; and c) Loop SWO [5] . . . . . . . . . 14

$2.524 \mathrm{GHz}, 1.2-\mathrm{V}$ CMOS RTWO TL Signals w.r.t. Ground . . . . . . . 16

2.6 Lumped Element RTWO with Proposed Phase Calibration Method [1] 17

3.1 Simulink ${ }^{\circledR}$ System Level RTWO Phase Calibration Circuit . . . . . . 24

3.2 1.2 V Rail-to-Rail RTWO Differential Output Clock Signals (Point A) 25

3.3 Output Phases (Point F) Before Calibraton - Top; After Calibration -

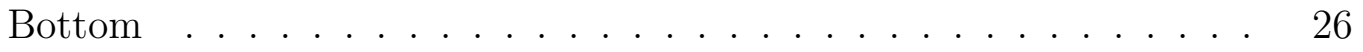

3.4 CML XOR Phase Detect - Phase in to Duty Cycle Out (Points B \& C) 27

3.5 Phase Measurement and Corresponding RTWO Delay Corrections Signals (Points D \& E) . . . . . . . . . . . . . . . . . . . . 28

3.6 Simulink ${ }^{\circledR}$ Implementation of a Nomially 24 MHz RTWO . . . . . . . 31

3.7 Simulink ${ }^{\circledR}$ FFT at RTWO Output (Point A) . . . . . . . . . . . . . . 34 
3.8 Simulink ${ }^{\circledR}$ Algorithm Blocks . . . . . . . . . . . . . . . . . . 35

3.9 Simulink ${ }^{\circledR}$ RTWO Phase Alignment Algorithm Flow Chart . . . . . . 37

3.10 Cadence ${ }^{\circledR}$ Virtuoso ${ }^{\circledR}$ AMS Designer System Level RTWO Phase Calibration Circuit . . . . . . . . . . . . . . . . . 38

3.11 RTWO Differential Output Clock Signals (Point A) . . . . . . . . 40

3.12 Output Phases (Point D) Before Calibraton - Top; After Calibration -

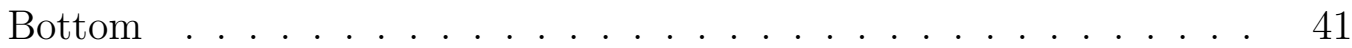

3.13 CML XOR - Phase in to Duty Cycle Out (Points B \& C) . . . . . . 42

3.14 Phase Measurement Output Voltages . . . . . . . . . . . . . . . . 44

3.15 Lumped Element RTWO based on Equations from $[6,7] \ldots \ldots$. . . 46

3.16 Digitally Controlled Ideal Capacitor Tuning Array . . . . . . . . . . 46

3.17 Cadence RTWO Phase Alignment Algorithm Flow Chart . . . . . . 49

4.1 CML XOR Function with Averaged (LPF) Outputs . . . . . . . . 51

4.2 CML XOR Transfer Function Including LPF . . . . . . . . . . . 52

4.3 Bipolar CML XOR Schematic . . . . . . . . . . . . . . 54

4.4 Bipolar CML SXOR Schematic . . . . . . . . . . . . 56

4.5 SXOR and XOR Output Duty Cycles for $90^{\circ}$ Inputs v. Frequency . . 57

4.6 SXOR and XOR Output Duty Cycles for $45^{\circ}$ Inputs v. Frequency .. 58

4.7 SXOR Input Signal Bias Network Schematic . . . . . . . . . . . . 59

4.8 SXOR ECL Output Transistor Schematic . . . . . . . . . . . . . 60

4.9 Layout of SXOR with ECL Outputs . . . . . . . . . . . . . 62

4.10 SXOR Phase Detector Version One Die $($ Phdetect_1) . . . . . . 63

4.11 SXOR Phase Detector Version Two Die (Phdetect_2) . . . . . . . 63

5.1 Simulation Test Bench for Phase Detector . . . . . . . . . . . . 64

5.2 Monte Carlo Results for $45^{\circ} \pm 10^{\circ}$, Fast, Extracted . . . . . . . 66

5.3 Monte Carlo Results for $45^{\circ} \pm 10^{\circ}$, Slow, Extracted . . . . . . . 69 
5.4 Monte Carlo Results for $90^{\circ} \pm 10^{\circ}$, Fast, Extracted . . . . . . . . . 70

5.5 Monte Carlo Results for $90^{\circ} \pm 10^{\circ}$, Slow, Extracted . . . . . . . . 71

5.6 AMSD System Model of Correctly Working RTWO Phase Calibration Circuit . . . . . . . . . . . . . . . . . . 74

5.7 Lumped Element RTWO LT [6,7] with Kit Varactors [8] . . . . . . . 75

5.8 Differential C, Q and R for VcapDnMOSNW Kit Varactor . . . . . . 75

5.9 Simulated Left, Bottom, Right and Top Signals of Figure 5.6 . . . . . 77

5.10 Simulated Varactor Control Voltages of Figure $5.6 \ldots$. . . . . . 78

5.11 AMSD System Model of Implmented RTWO Phase Calibration Circuit 80

5.12 Simulated Left, Bottom, Right and Top Signals of Figure 5.11 . . . . 81

5.13 Simulated Varactor Control Voltages of Figure 5.11 . . . . . . . . . . 82

5.14 Simulated Left, Bottom, Right and Top Signals of Modified Figure 5.6 83

5.15 Simulated Varactor Control Voltages of Modified Figure 5.6 . . . . . 84

6.1 Single-Ended Input to Differential Output Buffer . . . . . . . . . . . 86

6.2 Low Pass Filter $(\mathrm{LPF}) \ldots \ldots$. . . . . . . . . . . 86

6.3 Differential Phase Detector (Phdetect_2) Test Device Layout . . . . . 88

6.4 Differential Phase Detector Lab Test Set-up . . . . . . . . . . . . . . 90

6.5 Test Cable Delay De-Embedding Set-up . . . . . . . . . . . . . 95

6.6 Probe Delay De-Embedding Set-up . . . . . . . . . . . . . . . . 96

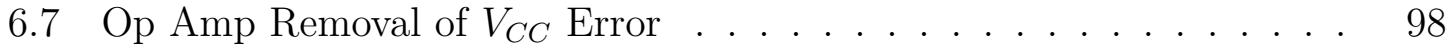

6.8 Phase Detector Transfer Function - 12.0 GHz, Measured . . . . . . . 99

6.9 Phase Detector Transfer $45^{\circ} \pm 10^{\circ}$ and $90^{\circ} \pm 10^{\circ}-12.0 \mathrm{GHz}$, Measured 100

6.10 Tuneable RTWO Lab Test Set-up . . . . . . . . . . . . . . . . . . . . 110

6.11 RTWO Phase Alignment Test Die (DUT Figure 6.10) . . . . . . . . . 111

6.12 Test Board 1 Phase Detector Output Signals . . . . . . . . . . . . . 113

6.13 Test Board 1 Phase Detector Output Signals . . . . . . . . . . . . . . 114 
6.14 Test Board 2 Phase Detector Output Signals . . . . . . . . . . . . . . 115

6.15 Test Board 2 Phase Detector Output Signals . . . . . . . . . . . . . 116

6.16 Test Board 2 Phase Detector Output Signals . . . . . . . . . . . . . . 117

A.1 RTWO Back-to-Back Gain Block . . . . . . . . . . . . . . . . 129

A.2 RTWO Variable Integer Delay Block . . . . . . . . . . . . . . 131

A.3 RTWO 45 Degree Section . . . . . . . . . . . . . . . . 131

A.4 CML Buffer for RTWO Phase Taps . . . . . . . . . . . . . . . . . . . 132

A.5 Differential In to Single-Ended Out Buffer . . . . . . . . . . . . 132

A.6 Single-Ended Buffer . . . . . . . . . . . . . . . . . . . . . . . 133

C.1 Phase Detector Test Card Schematic . . . . . . . . . . . . . . . . . . 158

C.2 Phase Detector Test Card BOM . . . . . . . . . . . . . . 159

C.3 Phase Detector Test Card Layout - Primary Side . . . . . . . . . . . 159

C.4 Phase Detector Test Card Layout - Secondary Side . . . . . . . . . . 160

C.5 RTWO Tuning Test Card Schematic - Page $1 \ldots \ldots 161$

C.6 RTWO Tuning Test Card Schematic - Page 2 . . . . . . . . . . . . . 162

C.7 RTWO Tuning Test Card BOM . . . . . . . . . . . . . . . . . 163

C.8 RTWO Tuning Test Card Layout - Primary Side . . . . . . . . . . . . 164

C.9 RTWO Tuning Test Card Layout - Secondary Side . . . . . . . . . . 165 


\section{Nomenclature}

\begin{tabular}{|c|c|}
\hline 16PSK & 16 Phase Shift Keying \\
\hline $32 \mathrm{PSK}$ & 32 Phase Shift Keying \\
\hline 8PSK & 8 Phase Shift Keying \\
\hline $\mathrm{ADC}$ & Analogue to Digital Converter \\
\hline ADPLL & All Digital Phase Locked Loop \\
\hline AMS & Analog Mixed Signal \\
\hline AMSD & Analog Mixed Signal Designer \\
\hline BBPD & Bang Bang Phase Detector \\
\hline BiCMOS & Bipolar/Complementary Metal-Oxide Semiconductor Technology \\
\hline BPSK & Binary Phase Shift Keying \\
\hline $\mathrm{C}$ & Capacitance \\
\hline $\mathrm{C}_{\mu}$ & Base to collector capacitance of a bipolar transistor \\
\hline $\mathrm{C}_{\mathrm{CB}}$ & Collector to Bulk Capacitance of a bipolar transistor \\
\hline CDR & Clock and Data Recovery \\
\hline CMOS & Complementary Metal-Oxide Semiconductor \\
\hline $\mathrm{CW}$ & Continuous Wave \\
\hline $\mathrm{C}_{\pi}$ & Base to emitter capacitance of a bipolar transistor \\
\hline $\mathrm{DAC}$ & Digital to Analogue Converter \\
\hline $\mathrm{DCO}$ & Digitally Controlled Oscillator \\
\hline DTI & Deep Trench Isolation \\
\hline DUT & Device Under Test \\
\hline DVM & Digital Volt Meter \\
\hline ECL & Emitter Coupled Logic \\
\hline FCW & Frequency Control Word \\
\hline FD & Fractional Divider \\
\hline
\end{tabular}




$\begin{array}{ll}\text { FPGA } & \text { Field-Programmable Gate Array } \\ f_{\mathrm{T}} & \text { The frequency at which the current gain of a transistor is zero } \\ \text { FUS } & \text { Frequency Up-Sampling } \\ \text { GSG } & \text { Ground-Signal-Ground semiconductor probe } \\ \text { GSSG } & \text { Ground-Signal-Signal-Ground semiconductor probe } \\ I_{C} & \text { Collector Current of a bipolar transistor } \\ I_{E} & \text { Emitter Current of a bipolar transistor } \\ \text { IL } & \text { Insertion Loss } \\ K_{p h a s e} & \text { Phase detector gain or transfer function }[9] \\ \text { L } & \text { Inductance } \\ \text { LDO } & \text { Low Drop-Out voltage regulator } \\ \text { LPF } & \text { Low Pass Filter } \\ \text { MD } & \text { Multimodulus Divider } \\ \text { mimCap } & \text { Metal-Insulator-Metal Capacitor } \\ \text { NPORT } & \text { Cadence }{ }^{\circledR} \text { N port s-parameter block } \\ \text { NXOR } & \text { Exclusive NOR logical function } \\ \text { P1dB } & \text { The input power level of an amplifier at which its output power level, } \\ & \text { due to compression, is } 1.0 \text { dB below its expected small signal level. } \\ \text { PSK } & \text { Phase Shift Keying - digital modulation of carrier phase } \\ \text { PVT } & \text { Process, Voltage and Temperature variation } \\ \text { QAM } & \text { Quadrature Amplitude Modulation } \\ \text { QPSK } & \text { Quadrature Phase Shift Keying } \\ \text { R } & \text { Resistance } \\ \text { RF } & \text { Radio Frequency } \\ \text { RTWO } & \text { Rotary Travelling Wave Oscillator } \\ \text { SD } & \text { Standard Deviation } \\ \text { SPST } & \text { Single-Pole Single-Throw switch function } \\ \text { SWO } & \text { Standing Wave Oscillator } \\ \text { SXOR } & \text { Symmetrical Exclusive OR gate implementation }[10] \\ \text { TDC } & \text { Time to Digital Converter } \\ \text { TL } & \text { Transmission Line } \\ \text { TS } & \text { Time Sample } \\ \text { Vc } & \text { Control Voltage } \\ \text { VNA } & \text { Vector Network Analyser }\end{array}$


$V_{T}$

$\mathrm{XOR}$

$\alpha$

$\tau_{D}$

$\tau_{F}$

Semiconductor thermal voltage $\approx 26 \mathrm{mV}$ at room temperature Exclusive OR function

Alpha - $\alpha=I_{C} / I_{E}$ for a bipolar transistor

Propagation delay of a logic circuit [11]

Transistor forward transit time [11] 


\section{Chapter 1}

\section{Introduction}

\subsection{Motivation}

There is a growing demand for common use wireless digital communications systems to operate at high RF and now microwave frequencies. This trend has pushed oscillator and synthesizer design to where the Rotary Travelling Wave Oscillator (RTWO) is an appropriate implementation choice. One of the advantages an RTWO has over LC-oscillators is that it produces multiple phases of the operating frequency period. The availability of these phases allows system architects and designers to re-think conventional circuit implementations, some of which are discussed in Section 1.2.

The Rotary Travelling Wave Oscillator (RTWO) illustrated in Figure 1.1 consists of a continuous transmission line loop folded over on itself to form a crossover and a number of back-to-back inverter or gain elements. During steady-state oscillation a wave front travels around the loop in either a clockwise or anticlockwise direction. The instantaneous phases marked on Figure 1.1 (i.e., $0^{\circ}, 45^{\circ}, 90^{\circ} \ldots$ ) show a possible path of a single wave front revolution around the loop. The frequency of oscillation is established by this electrical length, which includes the guided wavelength of the transmission lines and the gain element reactive parasitics. 


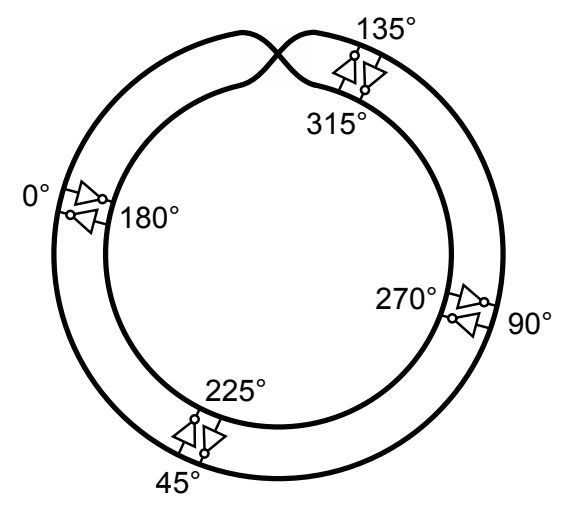

Figure 1.1: Idealized Illustration of a Four Section RTWO [1]

As the wave travels around the closed loop the transmission lines store energy and then pass it to the the gain elements. The gain elements replenish energy lost in the transmission lines and device resistances, and impose odd-mode or differential operation. The low loss and loaded-Q of the transmission lines allow the RTWO to have both relatively low power dissipation and low phase noise at microwave frequencies of oscillation.

Figure 1.1 illustrates that any phase relationship, from $0^{\circ}$ to $360^{\circ}$, can be extracted from the RTWO by tapping the travelling wave at relative points on the Transmission Line (TL) structure. This is a significant advantage over other types of oscillators. However, the accuracy of the phase relationship required for various applications is very difficult to achieve due to the inherent asymmetry of the RTWO TL structure (i.e., length of inner versus outer loop, and cross-over discontinuities) and device mismatch. That is, for a phase error of less than $5^{\circ}$ in a production environment with a satisfactory yield, lengthy design iterations and costly re-spins may be required [5], but in the end may not be enough.

The phase noise performance and power consumption of an RTWO is superior to that of a conventional LC-Oscillator as the frequency of operation increases past a 
few $\mathrm{GHz}$ (i.e., microwave frequencies) [5,12]. Additionally, as the frequency of operation increases the size of the RTWO decreases, which reduces the cost of fabrication. These as well as the phase tapping advantage mentioned earlier are significant motivation to justify research on finding ways to make RTWOs realizable in a production environment.

\section{$1.2 \quad$ RTWO Phase Tuning Applications}

The following paragraphs discuss some of the applications that can be improved by using multiple calibrated phases of an RTWO as a clock source. The two main sources of quantization error (non-linearity) in an All Digital Phase Locked Loop (ADPLL) are the Time to Digital Converter (TDC) and Digitally Controlled Oscillator (DCO). In Figure 1.2 the multiple phases of an RTWO DCO are used to reduce the quantization error in the feedback loop for a fractional divide ratio. A Bang-bang Phase Detector (BBPD) and Adaptive Loop Gain Controller replace the complexity and non-linearity of the TDC. Normally, fractional division ratios are created by alternating the ratio of the Multimodulus Divider (MD) between two consecutive integers, which can result in a large phase discontinuity in the divided clock. Here an RTWO DCO creates $m$ phases that are used by a fractional divider to reduce the large phase error that results from the changes in divide ratios at the MD. Within the fractional divider there is an integer divide ratio of $\mathrm{N}$. F phases are mulitplexed on $\mathrm{F}$ consecutive clock cycles of $f_{0}$. This reduces or increases the divider clock $\left(f_{\mathrm{CLK}}\right)$ time by $(F / N)\left(1 / f_{0}\right)$. Increasing $\mathrm{f}_{\mathrm{CLK}}$ cycle time will cause glitches that must be suppressed in the output clock. The Frequency Control Word (FCW) selects the $f_{0}$. The $\Sigma \Delta / \mathrm{SS}$ $(\Sigma \Delta /$ Spread Spectrum) block uses the FCW (integer + fraction) to modulate the divide ratios of the MD and Fractional Divider (FD) blocks to reduce and shape the 
quantization error (nonlinearity) in the fractional divide ratio $[2,13]$.

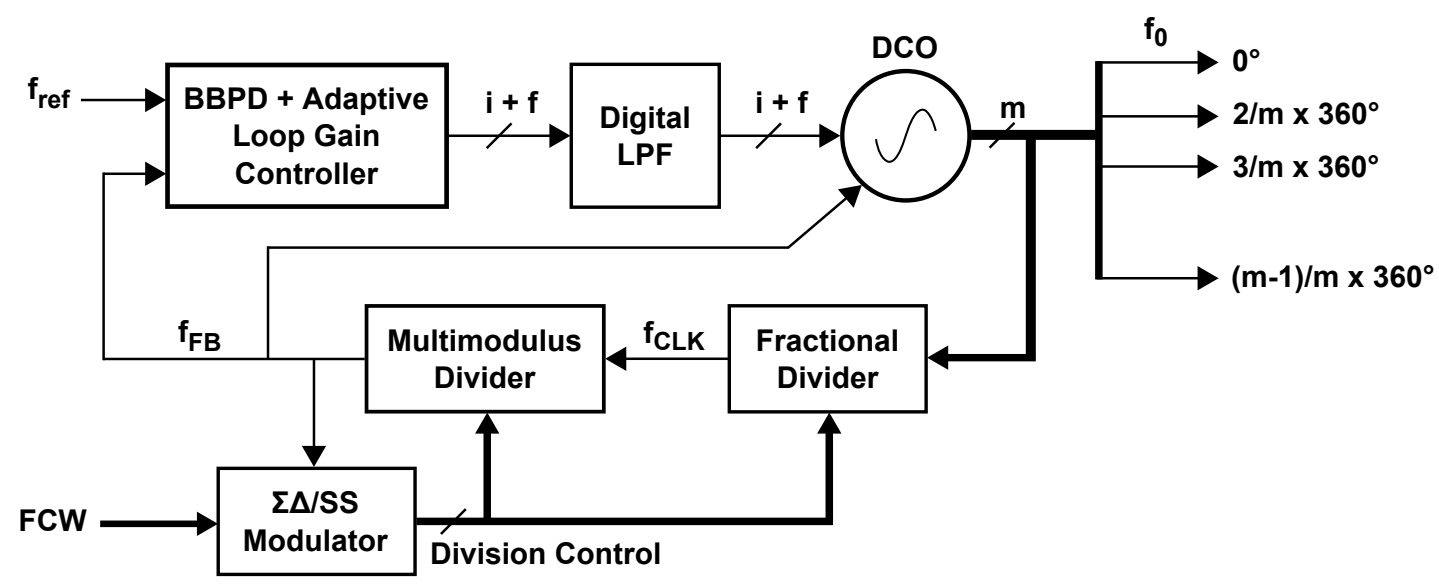

Figure 1.2: Fractional-N ADPLL with RTWO DCO [2]

Figure 1.3 shows a Digital Radio Polar Transmitter with an RTWO DCO [3] and Outphasing Modulator for a Software-Defined Transmitter [14]. The DCO generates the carrier frequency $f_{\mathrm{RF}}$ and the FCW (Frequency Control Word) selects RF channel. QAM-16 signals are generated using polar variables (amplitude - $\rho$ and phase - $\phi$ ). Phase modulation signals (i.e., BPSK, QPSK, 8PSK, 16APSK, 32APSK) can be generated modulating $\phi$ only, while amplitude modulation (QAM) and power setting require amplitude $(\rho)$ control. Grey Encoding can be used to reduce the bandwidth requirements for QAM signals. QAM-16 can be created using 12 phases and four amplitudes, while QAM-64 has 52 phases and 16 amplitudes. 


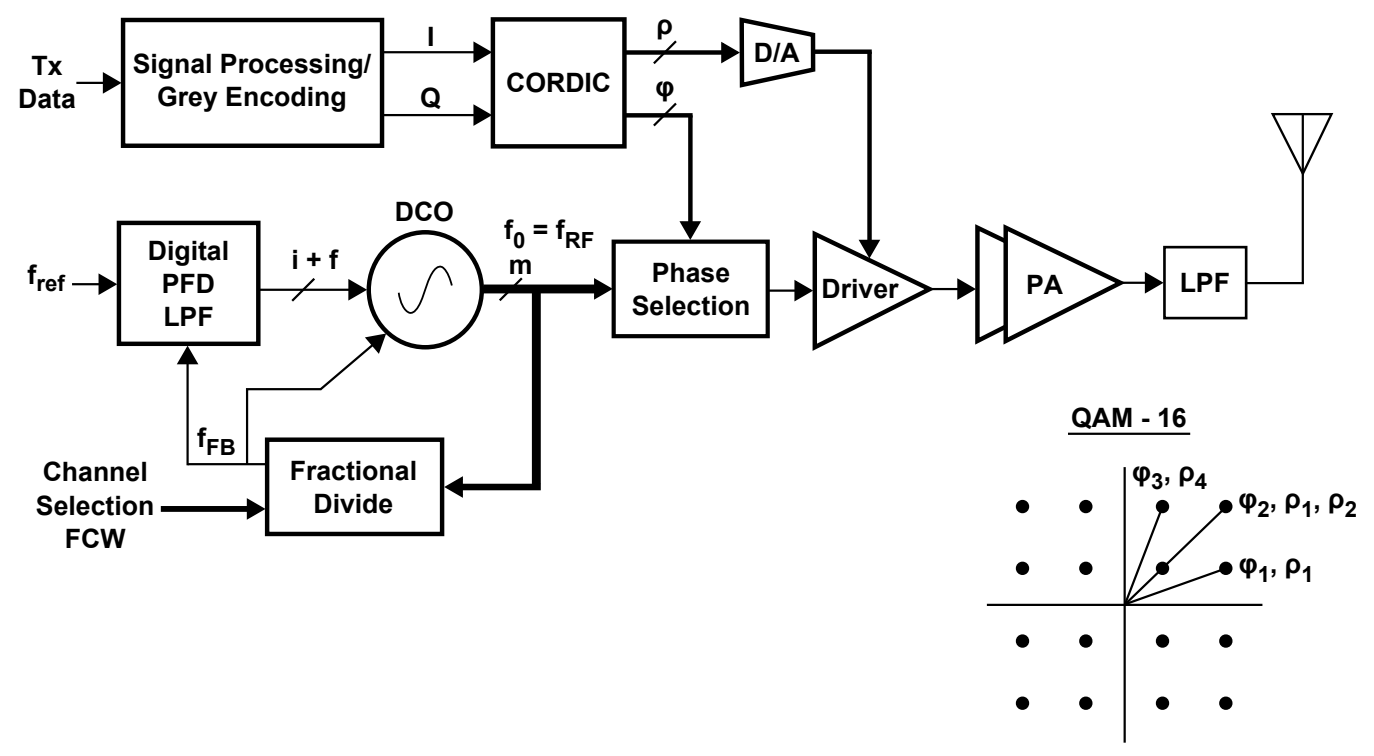

Figure 1.3: RTWO DCO in a Digital Radio Polar Transmitter [3]

Figure 1.4 illustrates a 6-Port Network Receiver that requires four phases of a DCO [4]. Here a DCO similar to that used in Figure 1.3 can be used to generate these signals.

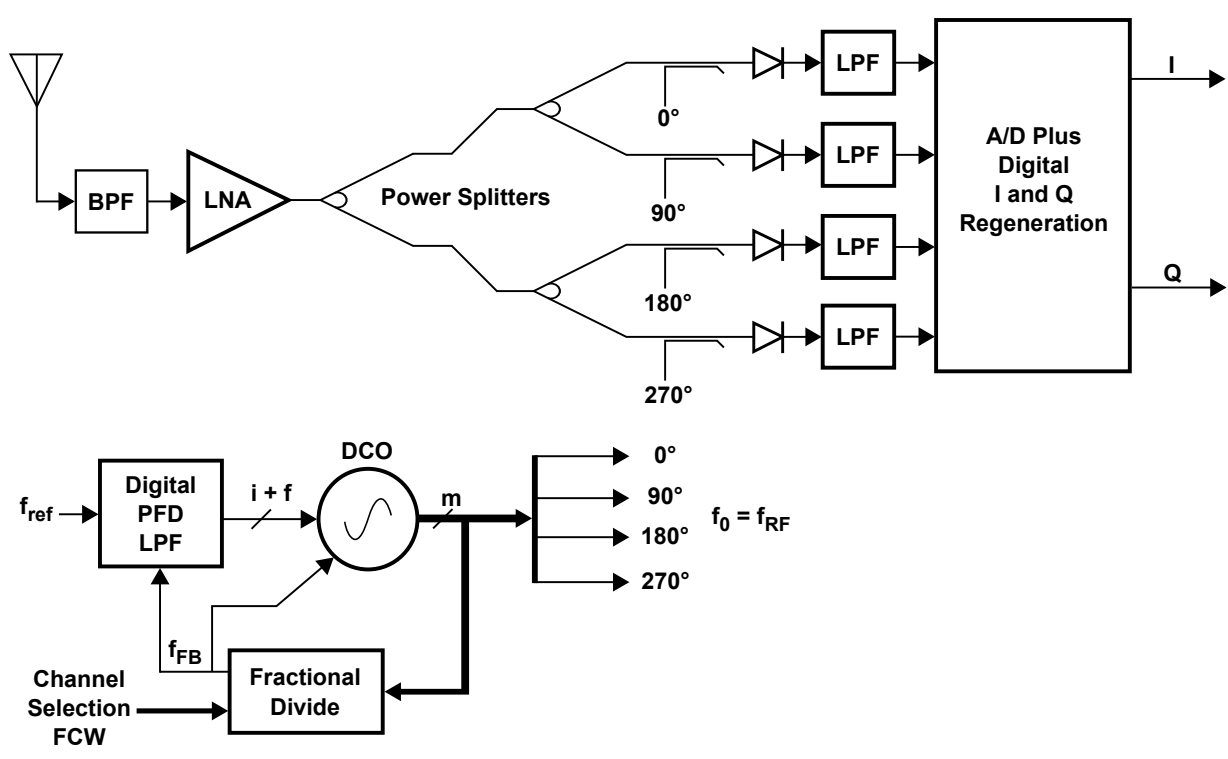

Figure 1.4: RTWO DCO in a 6-Port Network Receiver [4] 
Other applications whose performance at high frequencies could be improved by phase tuning of RTWO clock signals are Clock Distribution Networks [15], Phaseto-Digital Converter for an ADPLL [16] and oversampling circuits or phase tracking loops used in clock and Data Recovery (CDR) circuits for data alignment [17].

\subsection{Thesis Objectives and Scope}

The primary objective of this thesis is to demonstrate that the relative phases of oscillating signals tapped from an RTWO can be tuned to have an accuracy of better than $1^{\circ}$. This accuracy was selected as a good standard for image rejection in a digital radio. That is, for an image rejection of $40 d B$ the phase error between the I and Q oscillator signal needs to be less than $1.2^{\circ}$ and the amplitude error needs to be less than or equal to $2 \%[18]$.

It should be noted that this work does not include the development of an RTWO. The RTWO and single varactor elements for each transmission line section used in this thesis were developed by Kimia Ansari [6] and supplied as black-box component. The nominal operating frequency of this RTWO is $\approx 18 \mathrm{GHz}$.

This work also discusses some limitations of CMOS and Bipolar technologies for the implementation of high speed phase detectors used to phase tune an RTWO. That is, an RTWO is able to oscillate at very high frequencies (i.e., $18 \mathrm{GHz}$ and $36 \mathrm{GHz}$ [19]) relative to the current switching performance of transistors implemented in that same technology. This makes phase measurement on a cycle-to-cycle basis very challenging. 


\subsection{Contributions}

The main contribution of this thesis is to demonstrate that fine tuning of relative phases of a RTWO operating at microwave frequencies can be carried out using simple phase detectors, voltage controlled varactors and a straight forward iterative calibration algorithm. In order to limit power dissipation, this calibration can be carried out during initial system power-on and thereafter periodically or after frequency changes.

Also contributed here are implementation details of RTWO phase calibration i.e., phase detection, technology selection, calibration of phase calibration circuits, impact of rotation direction on the calibration algorithm.

\subsection{Thesis Organization}

Chapter 1 (Introduction) discusses the reasons for carrying out this research, as well as research objectives, scope and some possible circuit applications. Contributions to industry are also briefly presented. Chapter 2 (RTWO Concepts) provides some technical background of the RTWO that is useful going forward and the basic RTWO phase tuning concept. Chapter 3 (RTWO Phase Calibration) presents system level proof of concept circuits and simulations of RTWO phase tuning, first using Matlab/Simulink and second using Cadence AMS Designer. Chapter 4 (Phase Measurement Circuit) describes the phase detector architecture selection and implementation, as well as the removal of unequal differential transit delays. Chapter 5 (RTWO Phase Tuning Verification) details the extracted simulation of both the SXOR phase detector and the phase tuning of an RTWO. Chapter 6 (Experimental Results) discusses the lab test set-ups, calibration procedures and test procedures used to gather measurement results from the SXOR phase detector test cards and the RTWO phase alignment test cards. A list of conclusions, contribution and recommendation is documented 
in Chapter 7 (Conclusions and Recommendations). Appendix A describes the subblocks used to implement the Simulink RTWO 24 MHz block; Appendix B contains three versions of phase alignment algorithm verilog code (i.e., Cadence, FPGA and Matlab); and Appendix C displays the schematics, layout and BOMs for the two test cards used in this work. 


\section{Chapter 2}

\section{RTWO Concepts}

\section{$2.1 \quad$ Oscillator Fundamentals}

All systems designed to oscillate must, at minimum, satisfy the two requirements of the Barkhausen criteria. First, the circuit must include a feedback path that adds a delayed version of the forward signal, the feedback signal, to the forward path. The feedback signal must be added constructively to the forward path. This is illustrated in Figure 2.1.

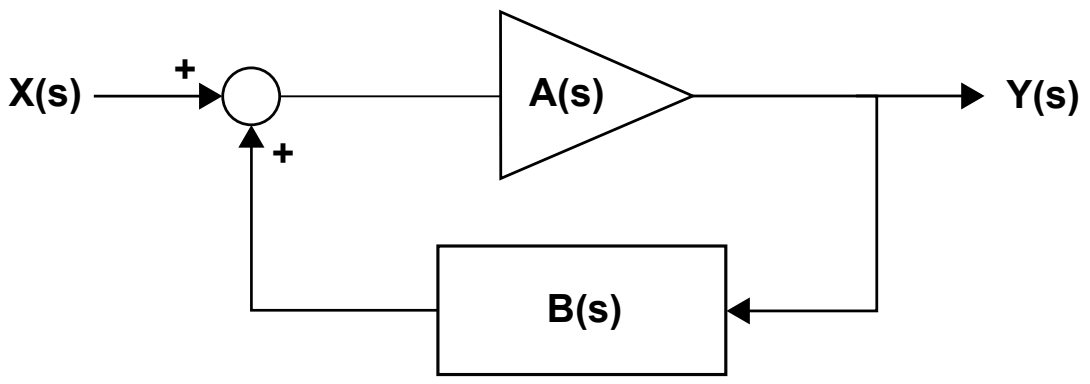

Figure 2.1: Classical Linear Feedback Model

Theoretically, a loop of wire or Transmission Line (TL) meets this criterion exactly, at every point along the loop, for a signal that oscillates with an integer number of guided wavelengths equal to the wire loop length. A Rotary Travelling Wave 
Oscillator (RTWO) is made by creating a twist in the TL loop (i.e., a figure eight) and folding one of the resulting loops inside the other, see Figure 2.2. This creates a differential structure that is one half the length of the differential guided wave path.

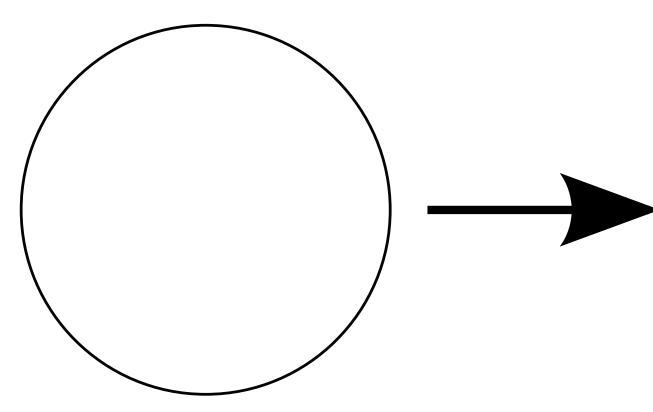

a)

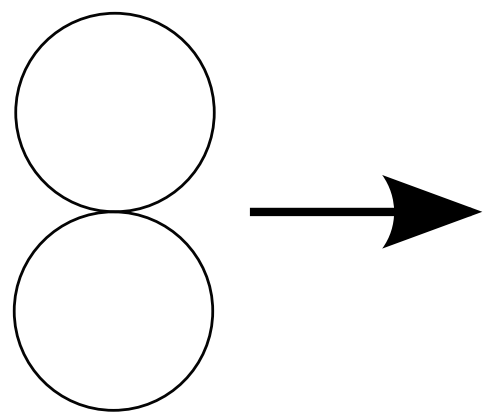

b)

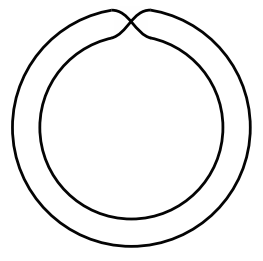

c)

Figure 2.2: [5] a) TL Loop; b) Twist; and c) Differential RTWO TL Structure

Secondly, after a circuit has reached steady state oscillation, the closed loop gain must be equal to 1.0. It should be noted that to ensure oscillation is initiated at startup, grows to steady-state and is sustained, the open loop gain must be greater than 1.0. This allows a very small signal perturbation (i.e., circuit noise) to be amplified to overcome circuit losses and build into an oscillating signal. Once the closed loop system reaches steady state, the circuit operates in a non-linear fashion to suppress any gain in the circuit in excess of 1.0. Mathematically, this can be shown using (2.1) (i.e., the transfer function for the classical linear feedback model of Figure 2.1):

$$
H(s)=\frac{Y(s)}{X(s)}=\frac{A(s)}{1-B(s) A(s)}
$$

Here, for the gain $B(s) A(s)=1.0, H(s)=\infty$; therefore, the signal will be compressed into non-linearity.

Gain in the feedback path of an RTWO is achieved by inserting a number of back-to-back -Gm elements between differential points on the RTWO structure, as 
illustrated in Figure 2.3. This structure of TL sections and gain elements (e.g., four -Gm sections, $\mathrm{N}=4$ ) also forces the system to operate differentially (odd mode). The RTWO rendering in Figure 2.3a) is somewhat theoretical since loops of finite TL width and equal circumference cannot fit inside one another. Figure 2.3b) is an RTWO layout with one cross-over, but a large difference between the inside loop and outside loop signal path lengths, and Figure 2.3c) is an RTWO layout with three cross-overs and a smaller loop signal path length difference then b). In a real implementation the placement of the gain stages in these layouts needs to be offset from what is shown below to maintain nominal $45^{\circ} \mathrm{TL}$ sections.

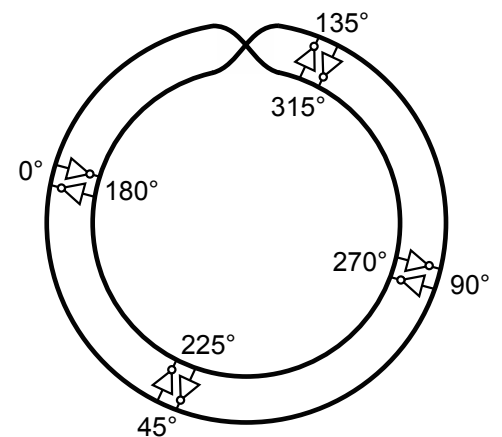

a)

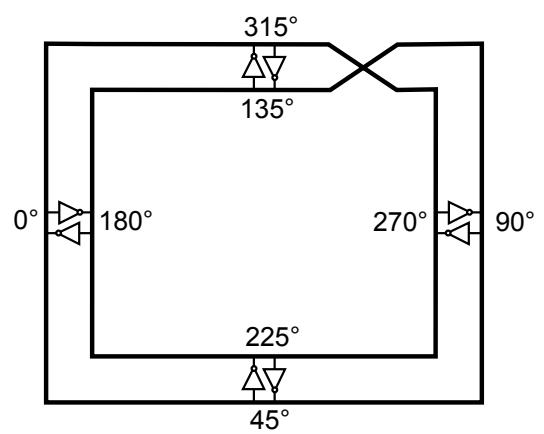

b)

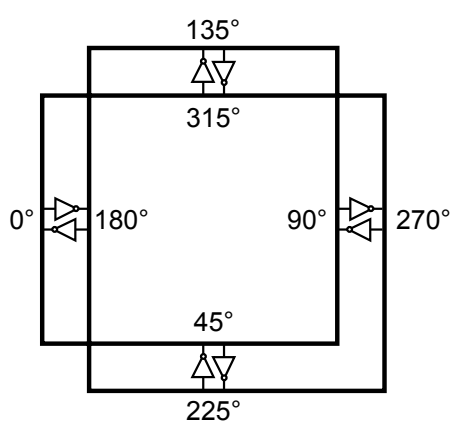

c)

Figure 2.3: a) Conceptual RTWO [1]; b) Single Cross-Over; c) Three Cross-Overs

\subsection{The Distributed Oscillator}

An RTWO can be considered a distributed amplifier special case (i.e., output fed back to input with a $180^{\circ}$ twist in the loop and satisfy Barkhausen's criteria) where the drain and gate parasitic capacitances of the differential gain stages are absorbed into the RTWO transmission lines $[5,20,21]$. This significantly raises the gain-bandwidth product of the gain stages, which allows the RTWO to have gain and oscillate at higher frequencies than would be expected from a conventional LC-oscillator of the same process. Theoretically, the operating frequency is limited by only the $f_{\mathrm{T}}$ of the 
amplifier circuit technology [15]. This is straight forward to simulate and has been realized in $130 \mathrm{~nm}$ CMOS at $18 \mathrm{GHz}$ and $36 \mathrm{GHz}$ in [19].

\subsection{RTWO Frequency Calculation}

The discussion that follows is a summary of concepts found in $[1,5]$, as well as other sources, and is included here to provide background understanding. After RTWO start up the steady-state wave front will propagate in the direction (i.e., either clockwise or anticlockwise) offering the lowest loss $[7,15]$. The phase velocity of the wave is $V_{p}=1 / \sqrt{L_{0} C_{0}}$, where $L_{0}$ and $C_{0}$ are the differential inductance and capacitance of the RTWO per unit length. In this thesis the RTWO is considered to be a differential structure and the signals that are extracted or tapped from it to be differential signals. If we select a differential position on the RTWO (e.g., differential tapping points $0^{\circ} / 180^{\circ}$ ), then the range of the full physical circumference of the RTWO is $180^{\circ}$ of phase relative to this physical starting point. The remaining $180^{\circ}$ of phase is realized by reversing the tapping points of the same physical starting position (i.e., $180^{\circ} / 0^{\circ}$ ) and travelling around the RTWO physical circumference again. Therefore, two laps of the RTWO are required to complete one cycle or wave length, $\lambda$, and for differential length $l$ we have a boundary condition $2 l=\lambda$ n, where $n=1,3,5 \ldots$. The fundamental frequency tends to be dominant because it receives the largest loop gain and losses are much higher for the harmonics. Thus, the RTWO frequency $f_{0}$ is given by [5]: 


$$
\begin{aligned}
f_{0} & =\frac{V_{p}}{2 l} \\
& =\frac{1}{2 l \sqrt{L_{o} C_{o}}} \\
& =\frac{1}{2 \sqrt{L C}} \\
& =\frac{1}{2 N \sqrt{L_{\text {diff }} C_{\text {diff }}}}
\end{aligned}
$$

where, $V_{p}$ is the phase velocity of the travelling wave, $L$ and $C$ are the total inductance and capacitance of the RTWO structure, $L_{\text {diff }}$ and $C_{\text {diff }}$ are the total differential inductance and capacitance of a TL section and $\mathrm{N}$ is the total number of sections so that [5]:

$$
\begin{gathered}
L=l L_{0}=N L_{\text {diff }} \\
C=l C_{0}=N C_{d i f f}
\end{gathered}
$$

These are the classical equations used to predict the fundamental oscillating frequency, $f_{0}$, of an RTWO.

\subsection{Superposition and Injection Locking}

The startup and steady state oscillation of an RTWO can be described as the superposition of a series of standing wave oscillators (SWO) [22] that will injection-lock [23] with each other to create a stable travelling wave oscillator $[1,5,24,25]$. Figure 2.4a) shows a $\lambda / 4$ SWO. This SWO is realized by a $\lambda / 4$ TL from the -Gm gain elements to the short circuit, the short produces a $-180^{\circ}$ reflection of the incident wave, and the $\lambda / 4$ TL from the short circuit to the -Gm gain elements produces the in-phase positive feedback required by Barkhausen. The phase is fixed with position, but 
the amplitude varies with position to produce a standing wave. Figure $2.4 \mathrm{~b}$ ) can be thought of as two $\lambda / 4$ SWOs connected at the $-\mathrm{Gm}$ elements to produce a $\lambda / 2 \mathrm{SWO}$ of the same frequency as Figure 2.4a). Following this train of thought, Figure 2.4c) is also a $\lambda / 2 \mathrm{SWO}$ formed by only considering a single set of -Gm elements of an RTWO with shorts at the Nulls. This TL loop is formed by unfolding and untwisting the RTWO TLs (i.e., the reverse of Figure 2.2).

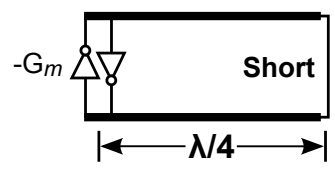

a)

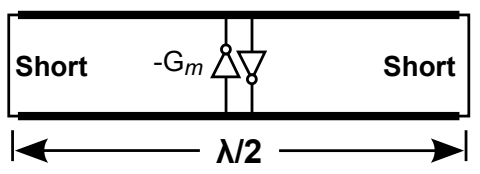

b)

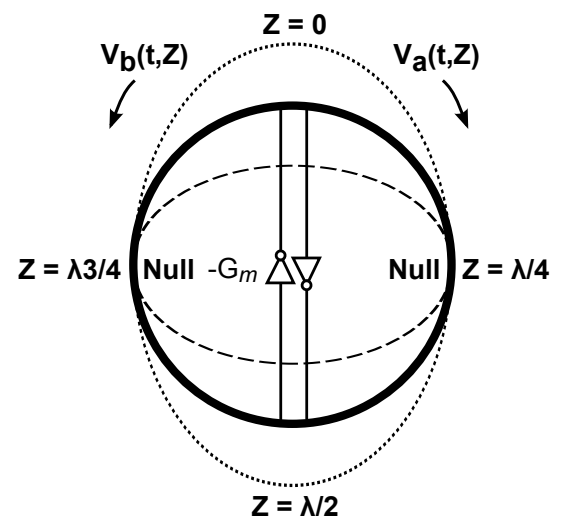

c)

Figure 2.4: a) $\lambda / 4$ SWO; b) $\lambda / 2$ SWO [5]; and c) Loop SWO [5]

In Figure 2.4c) the -Gm elements inject current into the TLs to produce a clockwise wave $\operatorname{Va}(t, Z)$ and a anticlockwise wave $V b(t, Z)$ as follows [5]:

$$
\begin{aligned}
& V_{a 0}(t, Z)=A_{0} \cos \left(\omega_{0} t-\beta z\right) \\
& V_{b 0}(t, Z)=B_{0} \cos \left(\omega_{o} t+\beta z\right)
\end{aligned}
$$

where $A_{0}$ and $B_{0}$ are peak voltage amplitudes, $\beta=2 \pi \lambda$ is the propagation constant and $\mathrm{z}$ is the physical position around the ring. The distribution of voltage along the ring is derived in [5] and shows that:

$$
V_{a 0}(t, Z)+V_{b 0}(t, Z)=2 B_{0} \cos \left(\omega_{o} t\right) \cos (\beta z)+\left(A_{0}-B_{0}\right) \cos \left(\omega_{o} t-\beta z\right)
$$


where the first term describes the behaviour of a standing wave and the second term describes the behaviour of a travelling wave. When the circuit is perfectly symmetrical $A_{0}=B_{0}$ only the standing wave will be present. The dashed lines in $2.4 \mathrm{c}$ ) illustrate the motion of the standing wave oscillation.

Now we consider that the N TL sections of a RTWO have a temporal offset of $T_{0} /(2 N)$, where $T_{0}=1 / f_{0}$, and a positional offset of $\lambda /(2 N)$. Using equation(2.10), the superposition of $\mathrm{N}$ standing waves (assuming perfect symmetry) gives the following equation $[5]$ :

$$
V_{0}(t, z)=\sum_{n=0}^{N-1}\left[2 B_{0} \cos \left\{\omega_{o}\left(t-\frac{n T_{o}}{2 N}\right)\right\} \cos \left\{\beta\left(z-\frac{n \lambda}{2 N}\right)\right\}\right]
$$

A more complete derivation of the following traveling wave equations is provided in [5]. The positional offset is $2 \lambda / N$ for anticlockwise equation:

$$
\begin{gathered}
V_{0}(t, z)=\sum_{n=0}^{N-1} B_{0} \cos \left(\omega_{o} t-\beta z\right) \quad \text { clockwise rotation } \\
V_{0}(t, z)=\sum_{n=0}^{N-1} B_{0} \cos \left(\omega_{o} t+\beta z\right) \quad \text { anticlockwise rotation }
\end{gathered}
$$

Equations (2.12) and (2.13) both describe travelling waves. The current injected by one set of -Gm elements into the TLs and next set of -Gm elements injection locks the SWOs and produces a travelling wave.

The simulated signals of Figure 2.5 are from the TL sections of an $\mathrm{N}=4$ RTWO (similar to Figure 2.3) implemented in a $130 \mathrm{~nm}$ CMOS process using CMOS buffers as -Gm elements and coupled TL models. The coupled TL sections have a length = $55 \mu \mathrm{m}$, width $=8 \mu \mathrm{m}$, separation $=16 \mu \mathrm{m}$, and the RTWO $f_{0}=24 \mathrm{GHz}$. Here start-up was initiated by a $1.0 \mathrm{~V}$ initial condition applied to the $0^{\circ} \mathrm{TL}$ section, so 
the $0^{\circ}$ and $180^{\circ}$ points begin to oscillate first as standing waves with the diagonally opposite points (i.e., $90^{\circ}$ and $270^{\circ}$ ) as nulls. Just prior to $4 \mathrm{~ns}$ this superposition of SWO behaviour injection locks into the travelling wave behaviour of an RTWO. With oscillators that do not require this initial condition at start-up this incipient standing wave mode is very short and the RTWO will achieve its travelling wave mode almost immediately. It should be noted that equation (2.10) predicts the peak amplitude of the standing wave mode oscillations should be twice that of the travelling wave mode oscillations. In this design the -Gm CMOS buffers will limit this amplitude to $1.2 \mathrm{~V}$.

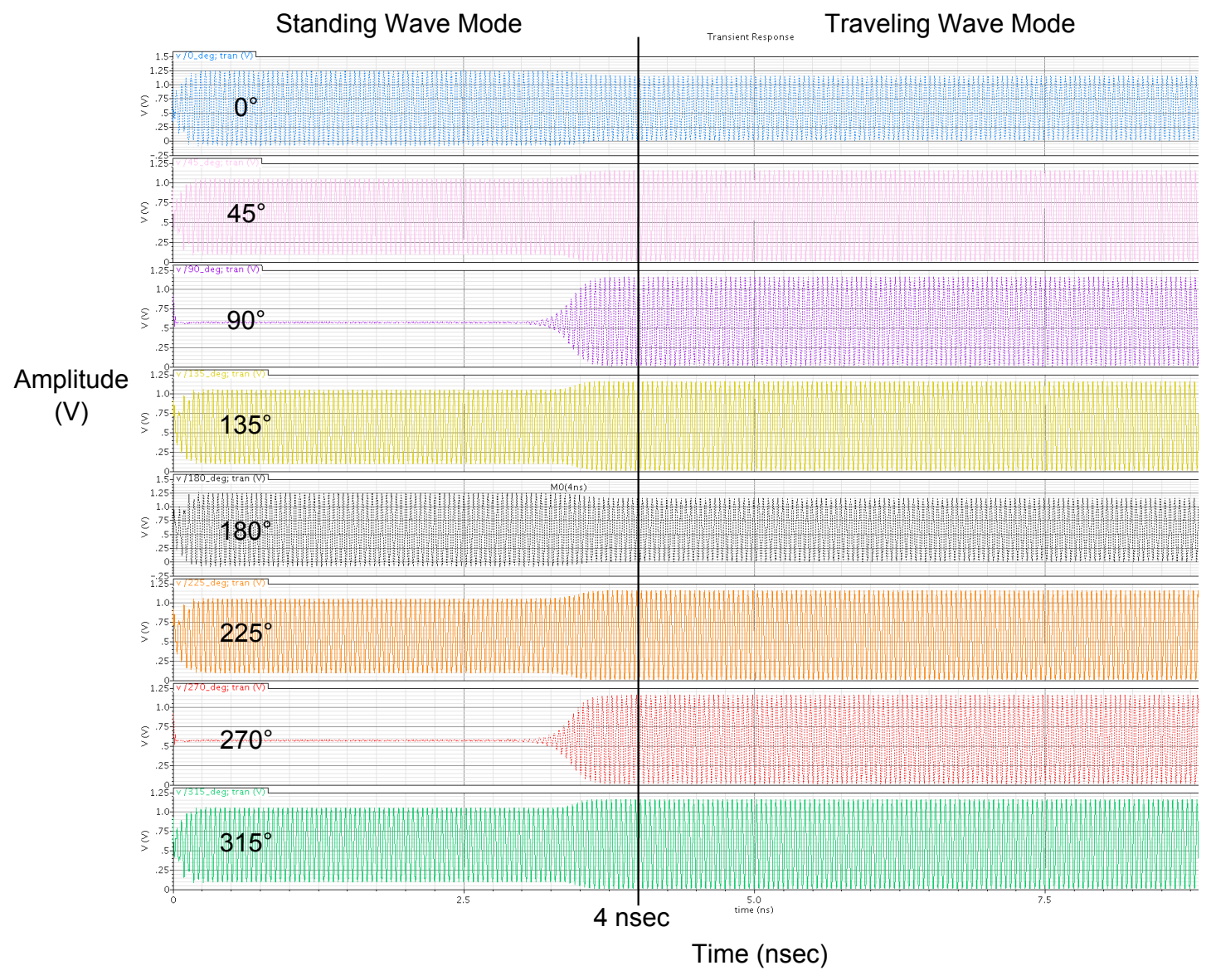

Figure 2.5: $24 \mathrm{GHz}, 1.2-\mathrm{V}$ CMOS RTWO TL Signals w.r.t. Ground 


\subsection{Phase Adjustment Concepts}

Phase adjustment of the RTWO is achieved by tuning the variable capacitance of individual line sections, which is realized with varactor banks connected across the TLs within each line section. This concept was identified in [1] and is summarized below with the aid of Figure 2.6.

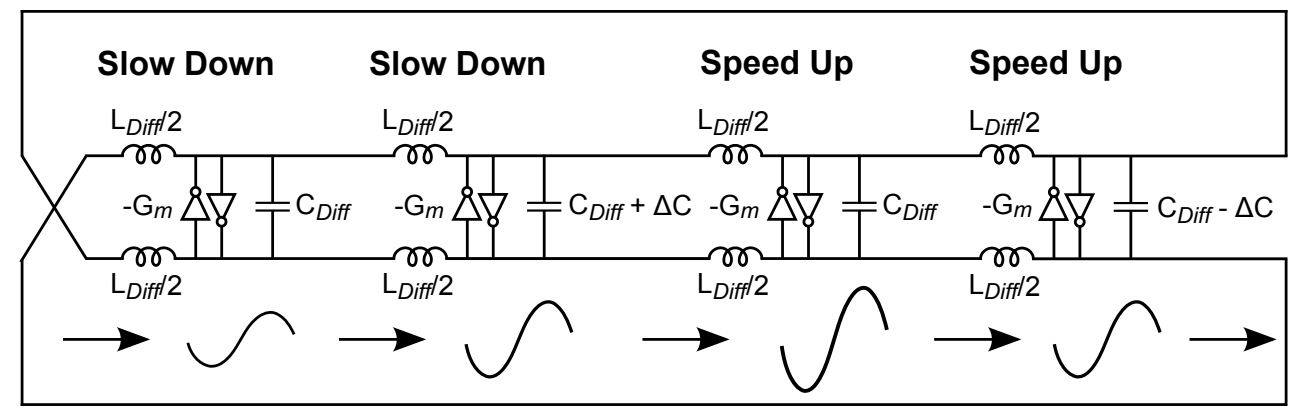

Figure 2.6: Lumped Element RTWO with Proposed Phase Calibration Method [1]

In Figure 2.6 the four TL sections of an RTWO have been modelled as lossless simplified lumped LC elements. $L_{D i f f}$ is the total differential inductance of each TL section. Each capacitive element represents the sum of the mutual capacitance of the differential TL section, the total associated driver NPN transistors capacitances (i.e., base to collector $C \mu$, base to emitter $C \pi$ and collector to bulk $C_{c B}[6]$ ), and the nominal variable capacitance of varactor banks installed in that TL section. The varactor capacitance of an individual TL section can be controlled independently of other TL sections to locally adjust the phase velocity of the travelling wave front. The example of Figure 2.6 shows that the travelling wave phase velocity is either slowing down or speeding up relative to the previous TL section for a wave front travelling from left to right. Here equal amounts of varactor capacitance $(\Delta \mathrm{C})$ are added to where the phase is leading and subtracted from where the phase is lagging so that phase is corrected, but the overall frequency of the RTWO remains constant. 
It should be noted that in this example both phase measurement and adjustment are independent of the direction of wave front rotation. A side effect of this method of phase tuning is that the amplitude of the travelling wave will vary around the RTWO as the impedance of individual TL sections is de-tuned.

If differential phase detectors are installed to measure the relative phase across each TL section, then an algorithm can be created to iteratively adjust the phases of TL sections as required and wait for the RTWO to settle. These iterations would continue until the phase across all TL sections was within a predefined tolerance, then the algorithm would stop. It is assumed that the purpose of the phase alignment algorithm is to tune all TL sections of an RTWO to have equal phase measurements. Any single phase detector measurement is relative to the other phase detector measurements carried out on a particular RTWO device. There is no requirement for any phase measurement to be absolutely accurate to an external standard.

\subsection{Phase Tuning Limits}

Reference [1] demonstrates mathematically that the clockwise or anticlockwise travelling wave modes of oscillation are the only stable states of the RTWO and it will not revert back to the standing wave mode, which is an unstable mode. However, this assumes that each SWO is injection locked at a common frequency. That is, as with any injection locked system there is both a minimum injection current requirement and injection frequency bandwidth beyond which locking will not occur. Adjusting the $\Delta C$ of individual TL sections changes the $V_{p}$ and frequency of those TL sections. As the frequency of adjacent TL sections approaches the injection locking limit, the phase noise will degrade and spurs will appear.

Reference [1] also documents a simulated injection lock range of $\Delta C= \pm 6 \%$ 
on the phase tuning of individual TL sections. Additionally, smaller changes of the $V_{p}(\Delta C)$ of individual TL sections produces signals of slightly different frequencies on the RTWO, which increases the phase noise of the system. Their fabricated prototype demonstrated $4 d B$ of phase noise degradation for a tuning range of more than $10^{\circ}$ (i.e., $\Delta C \approx \pm 2.5 \%$ ). The study of these limitations is beyond the scope of this thesis.

\subsection{Phase Change Calculation}

The lumped inductance $L_{\text {Diff }}$ and lumped capacitance $C_{D i f f}$ of Figure 2.6 form a Low Pass Filter (LPF) model of an RTWO TL section and are used as a starting point for deriving an equation that relates $\Delta C$ to phase. An ABCD parameter description of this LPF is as follows:

$$
\left|\begin{array}{ll}
A & B \\
C & D
\end{array}\right|=\left|\begin{array}{cc}
1 & Z_{L} \\
0 & 1
\end{array}\right|\left|\begin{array}{cc}
1 & 0 \\
Y_{C} & 1
\end{array}\right|=\left|\begin{array}{cc}
1+Z_{L} Y_{C} & Z_{L} \\
Y_{C} & 1
\end{array}\right|
$$

where $Y_{C}=j \omega C_{D i f f}$ and $Z_{C}=j \omega L_{D i f f}$. Using the conversion between two port network parameters (i.e., ABCD to s-parameter) found in [26], $s_{21}$ can be found from equation (2.14) to give:

$$
\begin{aligned}
s_{21} & =\frac{2}{1+Z_{L} Y_{C}+Z_{L} / Z_{o}+Y_{C} Z_{o}+1} \\
& =\frac{2}{2-\omega^{2} L C+j \omega L / Z_{o}+j \omega C Z_{o}}
\end{aligned}
$$


substituting $L=Z_{o}^{2} C$ into equation (2.15) we get:

$$
\begin{aligned}
s_{21} & =\frac{2}{2-\omega^{2} Z_{o}^{2} C^{2}+j 2 \omega Z_{o} C} \\
& =\frac{2\left(2-\omega^{2} Z_{o}^{2} C^{2}-j 2 \omega Z_{o} C\right)}{\left(2-\omega^{2} Z_{o}^{2} C^{2}\right)^{2}+\left(2 \omega Z_{o} C\right)^{2}}
\end{aligned}
$$

Solving $s_{21}$ for phase we get:

$$
\theta=-\arctan \left(\frac{2 \omega Z_{o} C}{2-\omega^{2} Z_{o}^{2} C^{2}}\right)
$$

Therefore, the change in phase for a change in variable capacitance in an RTWO TL section can be determined from:

$$
\Delta \theta=\arctan \left(\frac{2 \omega Z_{o}(C+\Delta C)}{2-\omega^{2} Z_{o}^{2}(C+\Delta C)^{2}}\right)-\arctan \left(\frac{2 \omega Z_{o} C}{2-\omega^{2} Z_{o}^{2} C^{2}}\right)
$$

Equation (2.18) is useful for estimating the change in phase of an RTWO TL section for a change in varactor capacitance in that RTWO TL section. A change in varactor capacitance is due to a change in varactor control voltage. Armed with these two relationships an estimate of the required correction voltage step size can be made, see Section 5.2. However, it should be noted that this equation gives, at best, a first order approximation. This is because an RTWO is a dynamic system where a single parameter change creates a new steady state. This equation only considers 
the starting point of a single TL section; not the resulting new steady state of the whole RTWO. That is, assuming TL sections are not isolated from one another, a capacitance change made in one TL section will change the electrical phase length in every TL section of a particular RTWO. An example of this behaviour is shown in Figure 3.14, where for any single phase adjustment the varactors of only two TL sections are adjusted. Yet the phase of all four TL sections changes to give the appearance that all four TL sections are being adjusted.

Additionally, when a TL section capacitance is changed its characteristic impedance also changes, which changes signal amplitude and the impedance transition between TL sections. The parameter levels under which injection locking to produce a travelling wave system (injection current and bandwidth) have also changed. Taken to an extreme, TL capacitance adjustment can cause frequency domain spurs to appear and degeneration to SWO operation (i.e., failure to injection lock) as discussed in Section 2.6. 


\section{Chapter 3}

\section{RTWO Phase Calibration}

\subsection{The System Concept}

Top-Down development has proven to be the design methodology for systems and circuits that results in predictable performance in the shortest development cycles. This begins with system implementation and simulation at the highest level of abstraction. System architects and designers will define the system as a group of functional blocks and interconnecting signals so they may write comprehensive specifications for these blocks and signals. Each block at a particular level of abstraction is made up of blocks and signals from the next lower level of abstraction. This approach promotes logical partitioning, as well as a complete and effective understanding of the system at any level of abstraction while design details at other levels are hidden until required. It also provides a starting point for bottom-up iterations that will help to refine the system specification to a practical and cost-effective realizable point.

This methodology may also be used to demonstrate a proof of concept. That is, to find the answers to questions like will the system work logically and mathematically, and does a particular implementation achieve the original goals. This chapter first

discusses the Matlab ${ }^{\circledR} /$ Simulink $^{\circledR}$ circuits and then the Cadence ${ }^{\circledR}$ Virtuoso ${ }^{\circledR}$ AMS 
Designer circuits that were created and simulated to demonstrate the concept of RTWO phase tuning.

\subsection{Proof of Concept - Matlab ${ }^{\circledR} /$ Simulink $^{\circledR}$}

Figure 3.1 is a functional block diagram of the system used to demonstrate the concept of RTWO phase tuning. The block RTWO $24 \mathrm{MHz}$ contains the RTWO divided into eight $45^{\circ}$ TL sections tapped to provide differential outputs $\phi 1 / \phi 2, \phi 3 / \phi 4, \phi 5 / \phi 6$ and $\phi 7 / \phi 8$. The inputs DlyCtl1 to DlyCtl4 are the variable delay control signals associated with each tapped differential TL section. The simulation frequency was reduced to $24 \mathrm{MHz}$ to shorten the real time duration of individual simulation runs. Parasitic components are not implemented in this model so this change in operating frequency does not detract from the demonstration. 


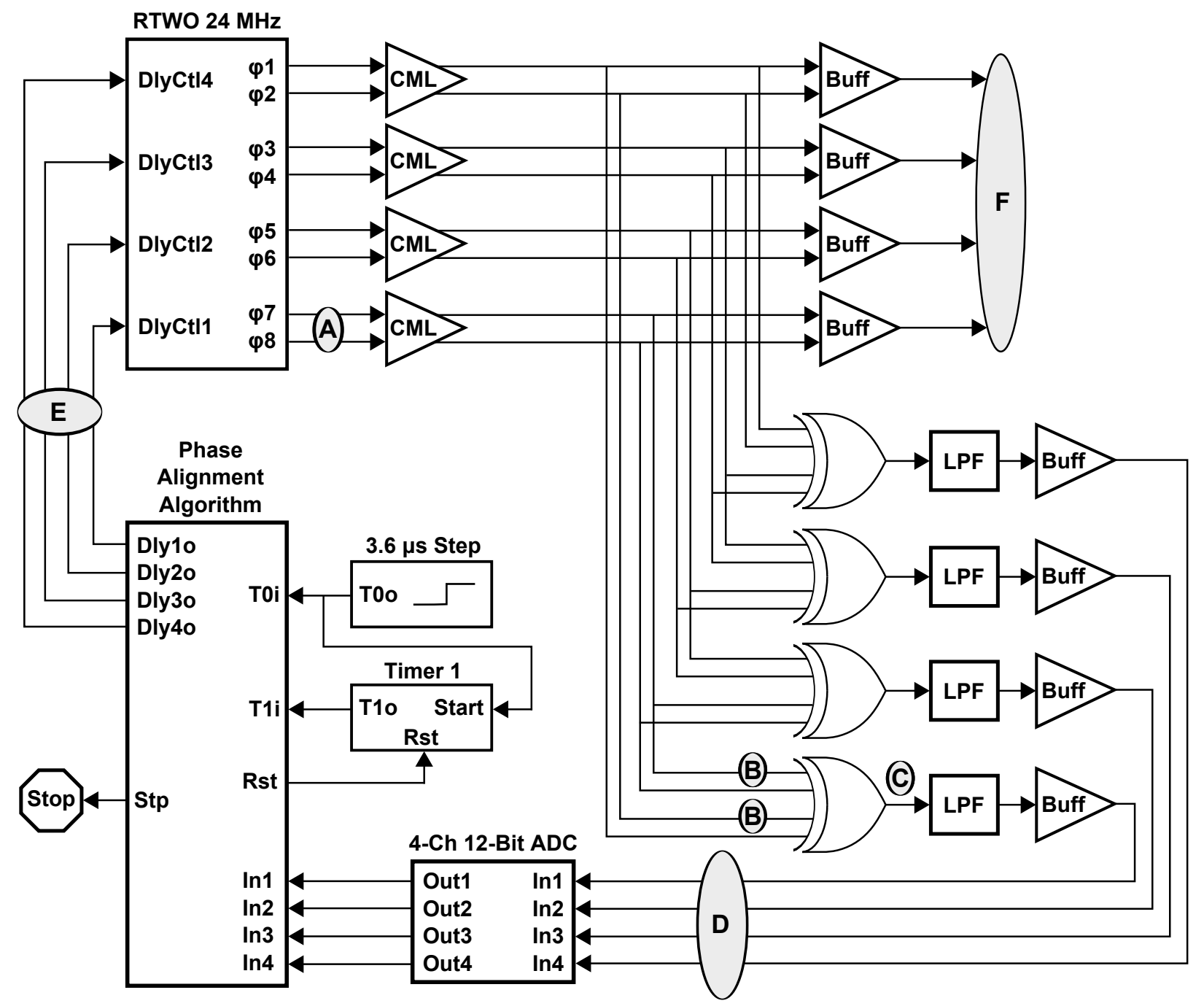

Figure 3.1: Simulink ${ }^{\circledR}$ System Level RTWO Phase Calibration Circuit

Point A of Figure 3.1 is a sample of the rail-to-rail differential output clock signals ( $\phi 7$ and $\phi 8)$ of the RTWO after it has reached steady-state oscillation, see Figure 3.2. 


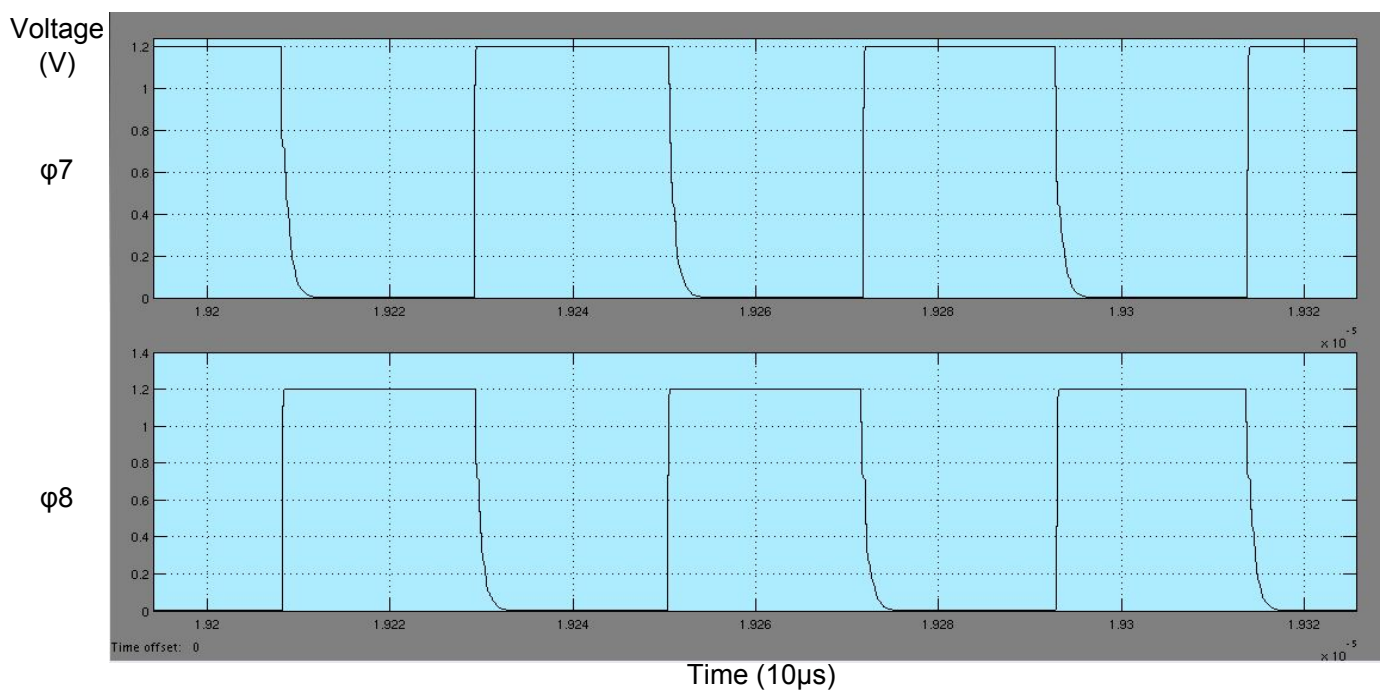

Figure 3.2: 1.2 V Rail-to-Rail RTWO Differential Output Clock Signals (Point A)

These 1.2 V rail-to-rail (i.e., CMOS voltage levels) clock signals are then converted to differential Current Mode Logic (CML) voltage levels (i.e., $0.85 \mathrm{~V} \pm 0.350 \mathrm{mV}$ ) using CML buffers. This conversion to CML was necessary because in a realizable circuit CMOS gates will not support signals operating at a frequencies in the order of $18 \mathrm{GHz}$. The CML clock signals are fed to differential to single ended buffers (Buff) and to XOR gates. The Buff block outputs, point F, show the clock phases with phase skew intentionally programmed in the RTWO as a starting point for the phase alignment simulation, labelled Before Calibration in Figure 3.3. Specifically, the phases marked $\Delta 1=59.6^{\circ}$, between $\phi 1 / \phi 2$ and $\phi 3 / \phi 4, \Delta 2=43.6^{\circ}$, between $\phi 3 / \phi 4$ and $\phi 5 / \phi 6, \Delta 3=47.2^{\circ}$, between $\phi 5 / \phi 6$ and $\phi 7 / \phi 8$, and $\Delta 4=29.6^{\circ}$, between $\phi 7 / \phi 8$ and $\phi 2 / \phi 1$. It should be noted that these four phase deltas will always sum to $180^{\circ}$ in an RTWO that has reached steady-state travelling wave oscillation. The signals of Figure 3.3, labelled After Calibration, show all these phase differences equal to $45^{\circ} \pm$ Limit, where Limit is a predefined resolution, see RTWO Phase Alignment Flow Chart Figure 3.9. Here the calibration algorithm has iteratively adjusted the phase deltas, determined that the phase error between all the measured signals is 
within a predefined resolution and has stopped the algorithm as no further adjustment was required.

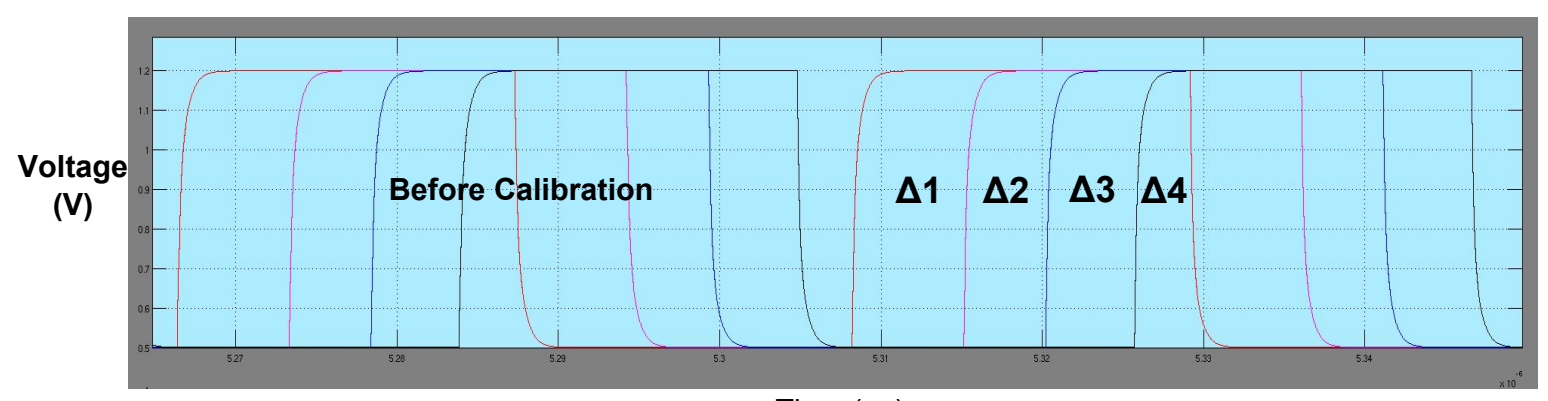

Time $(\mu s)$

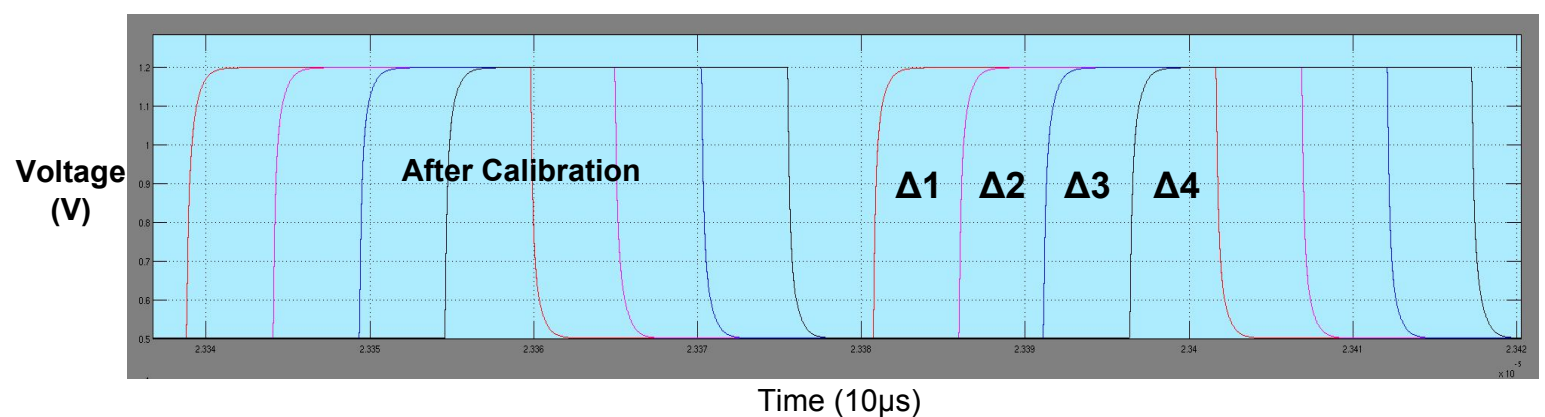

Figure 3.3: Output Phases (Point F) Before Calibraton - Top; After Calibration Bottom

\subsubsection{RTWO Phase Measurement}

In Figure 3.1 the differential clock signals representing the relative phases of the four differential TL sections of the RTWO are applied consecutively to the inputs of four XOR phase detectors. The XOR gates produce a duty cycle signal that is proportional to the phase taken up by each differential TL section. In Figure 3.4 we can see that the phase difference between signals $\operatorname{In} 1+$ and In $2+$ is represented by the duty cycle of the Out signal. 


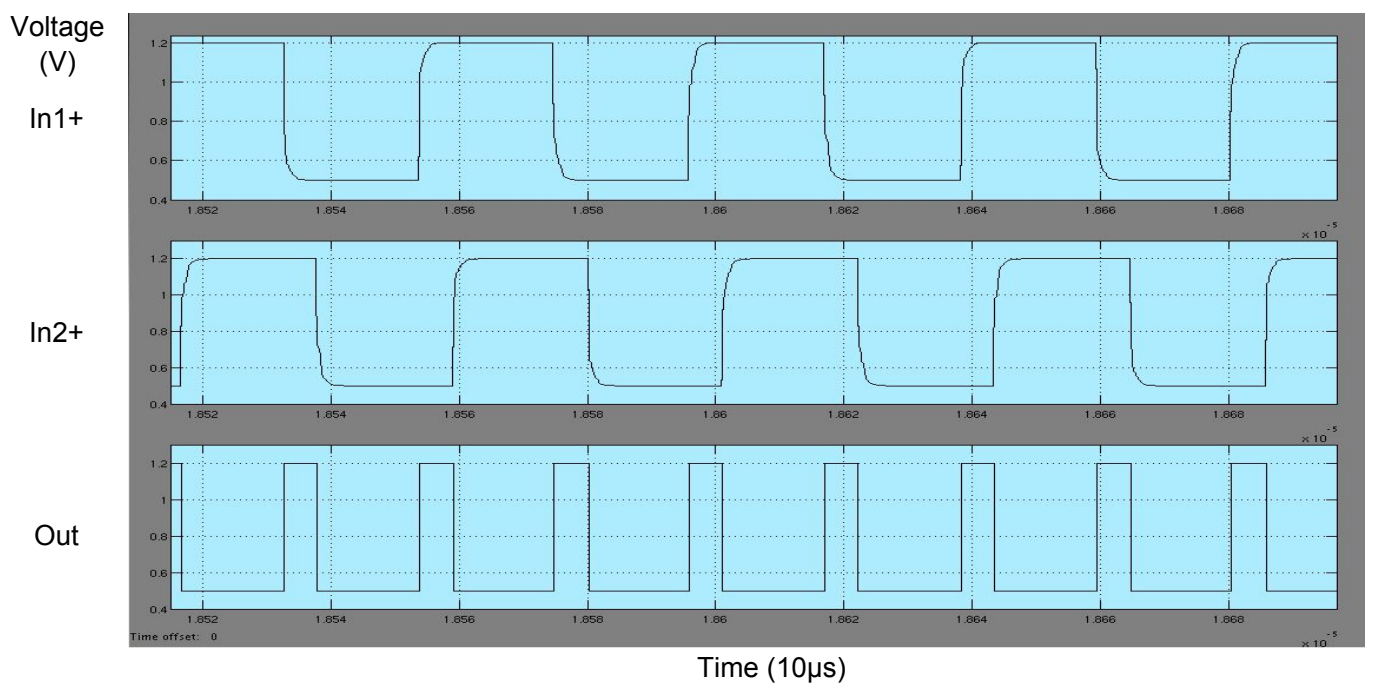

Figure 3.4: CML XOR Phase Detect - Phase in to Duty Cycle Out (Points B \& C)

It should be noted that the phase of the differential inputs feeding the bottom XOR from the top CML gate (i.e., $\phi 1, \phi 2)$ is reversed so the measurement will be over the single remaining TL section rather than the three TL sections already measured. In fact, this fourth XOR phase detector is redundant as this phase difference at steady-state can be determined by subtracting the sum of the other three measured phases from $180^{\circ}$ as follows:

$$
\Delta N=180^{\circ}-\sum_{n=1}^{N-1} \Delta n
$$

where for this RTWO $N=4$.

The outputs of these XOR phase detectors are single-ended CML (i.e., 0.85V \pm $0.350 \mathrm{mV}$ ) clock signals of twice the frequency of the input signals with output duty cycles proportional to the input phase differences. These duty cycles are averaged through a Low Pass Filters block (LPF), which consists of two cascaded single order LPFs with cut-off frequencies of $500 \mathrm{kHz}$ followed by $2.0 \mathrm{MHz}$. Next the Buff blocks applies a dc offset of $-0.6 V$ and a gain of five to produce the signals at point $\mathrm{D}$, 
i.e., Out1/4 of Figure 3.5. The amplitudes of these signals are proportional to the measured phases at each iteration of the phase alignment algorithm.

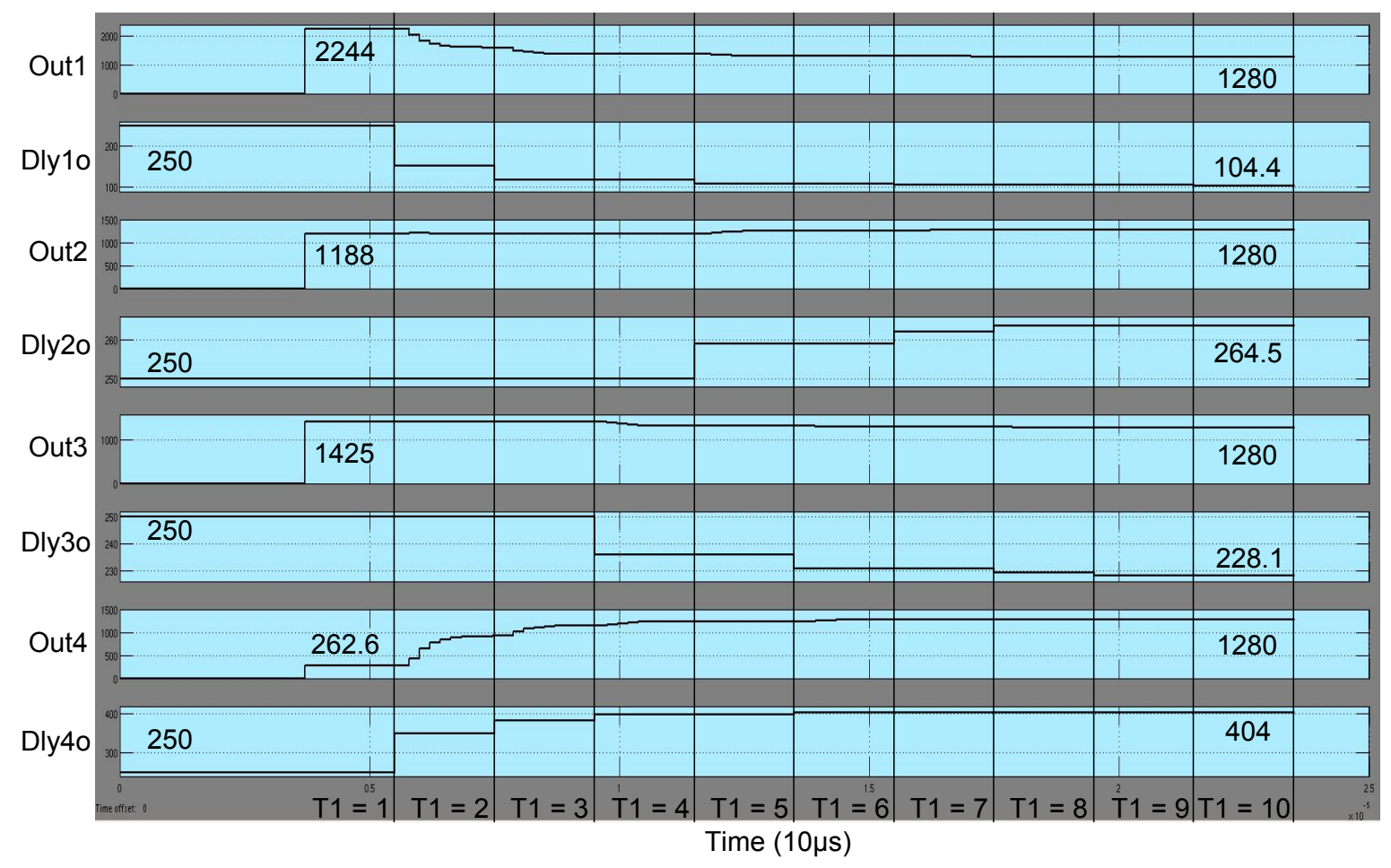

Figure 3.5: Phase Measurement and Corresponding RTWO Delay Corrections Signals (Points D \& E)

Next these signals are converted to binary numbers with a 4-Channel 12-Bit Analogue-to-Digital Converter (ADC). These numbers are passed to the Phase Alignment Algorithm block, which finds the TL section that has the largest and the TL section has the smallest phase measurement. It then uses this information to adjust variable delay signals Dly1/4o, which control the phase delay of each differential TL section of the RTWO. These functions are triggered every $20 \mu s$ by timer T1. This cycle time is set to be longer than the settling time of the RTWO (i.e., $<13 \mu s$ ) after a change in phase delay. Figure 3.5 illustrates these signals at point E for each cycle of T1 (i.e., iteration of the Phase Alignment Algorithm). In Figure 3.1 the 3.6 $\mathrm{s}$ Step block holds off Phase Alignment Algorithm at simulation start until after the 
RTWO $24 \mathrm{MHz}$ block has been initialized i.e., the initial TL section delays have been programmed, see Table 3.1.

The Out1/4 signals of Figure 3.5 show numbers that represent the measured phase at the beginning through to the end of the simulation. After the algorithm makes the first phase measurement, i.e., Out $1=2244$, Out $2=1188$, Out $3=1425$ and Out4 $=262.6$, it will adjust the delay of the largest and smallest phase delays using signals Dly1/4o. The algorithm (and simulation) will stop when all these numbers are within seven of each other, which is illustrated by the measurement results Out1 = Out $2=$ Out $3=$ Out $4=1280$.

Each channel of the 4-Ch 12-Bit ADC block of Figure 3.1 has a full range of $1.2 \mathrm{~V}$. The 12-Bit ADC plus the coding gain of 2.322 bits (i.e., a gain of 5 or $14 d B$ from the previous analogue blocks) gives an effective 14.322-Bit ADC, which results in the following measurement resolution:

$$
\begin{gathered}
1.2 /\left(2^{14.322}-1\right)=58.6 \mu V \\
180 /\left(2^{14.322}-1\right)=8.79 \mathrm{~m}^{\circ} \\
58.6 \mu V / 8.79 m^{\circ}=6.67 m V /^{\circ}
\end{gathered}
$$

This result can be stated more simply from the full-scale voltage and full-scale phase as follows:

$$
1.2 \mathrm{~V} / 180^{\circ}=6.67 \mathrm{mV} /^{\circ}
$$

The algorithm stop resolution of seven mentioned earlier is equivalent to $7 \times$ $8.79 \mathrm{~m}^{\circ}=61.53 \mathrm{~m}^{\circ}$. At the same time this coding gain reduces the maximum measurement range to $180^{\circ} / 5=36^{\circ}$. 


\subsubsection{The Simulink ${ }^{\circledR}$ RTWO Block}

Figure 3.6 is the RTWO $24 \mathrm{MHz}$ block at the next lower level of abstraction. Here we see four distinct TL sections made up of Gain, Delay and $45^{\circ}$ Section blocks each delineated by differential clock signal tapping points. Also visible are the TL section delay control inputs (i.e., DlyCt11/4), which determine the phase delay of blocks Delay 1 to Delay 4. Gain 1 to 4 provide the back-to-back or differential gain that supports oscillation in the RTWO, see Figure 2.3. $45^{\circ}$ Section 1 to 4 insert nominal loss, nominal $45^{\circ}$ phase delay and a LPF characteristic into the TL sections. The $0.2 \mathrm{~V}, 0.2 \mathrm{~ns}$ Step block provides a signal perturbation to disrupt the steady-state condition of the RTWO and initiate the build up of an oscillating signal in the loop at simulation start. 


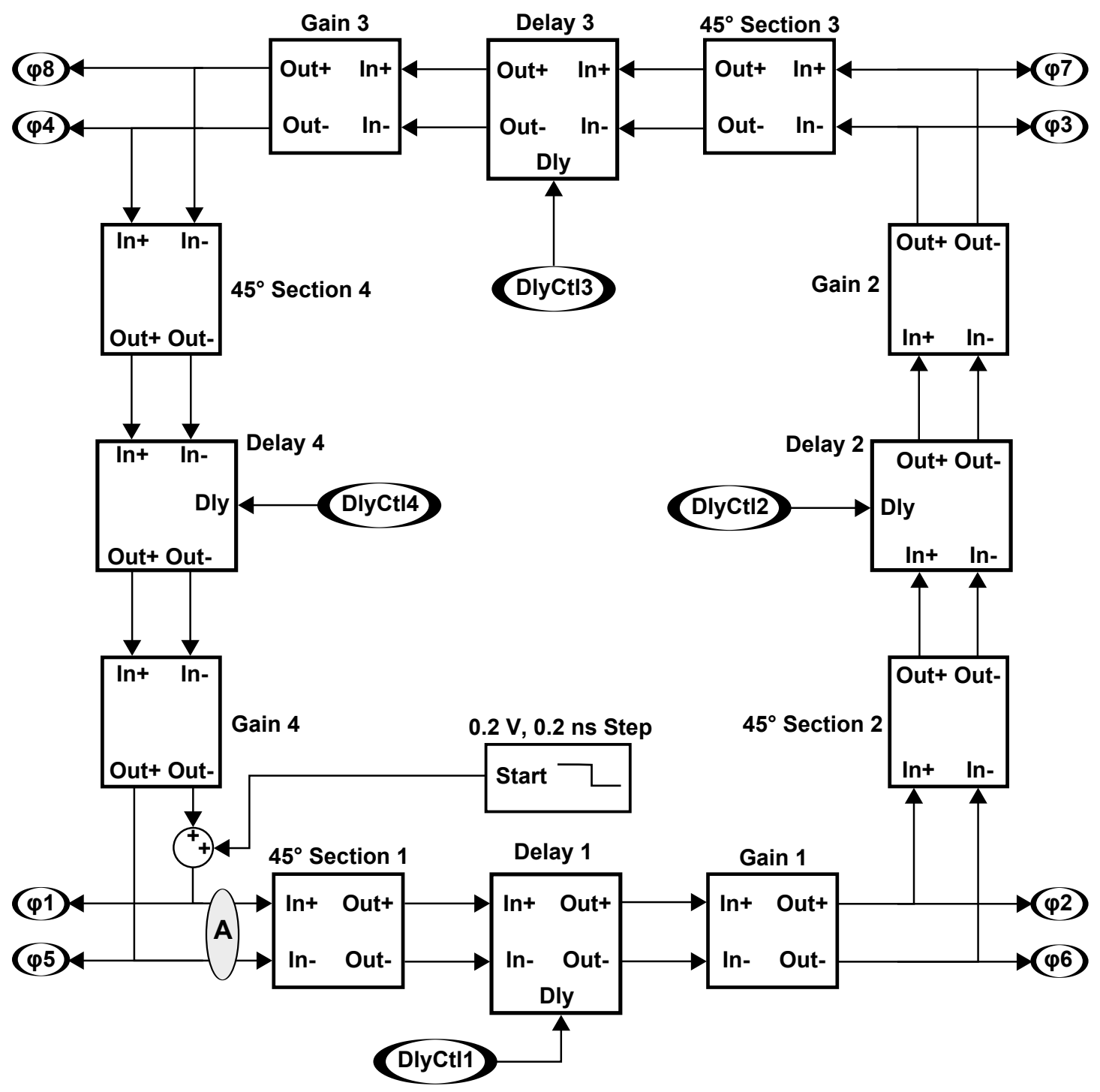

Figure 3.6: Simulink ${ }^{\circledR}$ Implementation of a Nomially $24 \mathrm{MHz}$ RTWO

Prior to running the Simulink ${ }^{\circledR}$ model a Matlab ${ }^{\circledR}$ file, setup.m, must be run to initialize the simulation Time Step (TS) and other variables used in the model (i.e., FUS, Freq, Loss, alpha, slew and offset_n). The 0.2 V, 0.2 ns Step block in the Simulink ${ }^{\circledR}$ model, Figure 3.6, is where this TS is established and other blocks in the system inherit this parameter form this block. The TS is calculated follows:

$$
T S=1 /(\text { FUS } \times \text { Freq })
$$


where, $F U S=3600$ and Freq $=24 \times 10^{6}$.

Variable Frequency Up Sampling (FUS) establishes that there will be 3600 TSs per cycle at the nominal operating frequency of $24 \mathrm{MHz}$. It should be noted that the frequency of the RTWO is not determined by these variables. The frequency of a particular RTWO simulation is controlled by the delay elements in its TL paths.

The delay of the four differential TL sections is initialized to produce the phase error or skew that will be corrected by the phase alignment algorithm during simulation. The initial phase skew is set up in the $45^{\circ}$ Section blocks of Figure 3.6 using setup.m, i.e., $Z^{-(F U S / 8)+o f f s e t \_n}$ in Tables 3.1 and 3.2. In Figure 3.5 we see that after the calibration algorithm begins it initializes the variable Delay blocks to 250 TSs so both increases and decreases in the delay tuning can be made to this value. The rows across Table 3.1 show the total delay and resulting skewed phases for each TL section at initialization, i.e., Variable Delay Block + FUS $/ 8+O f f s e t \_n=$ Total $(T S)$ for a TL section.

Table 3.1: Transmission Line Phase Delay at Simulation Start

\begin{tabular}{|c|c|c|c|c|c|}
\hline \multirow{2}{*}{ TL Section } & \multicolumn{4}{|c|}{ Initial 45 ${ }^{\circ}$ TL Section (TS) } & \multirow{2}{*}{ Phase $\left(^{\circ}\right)$} \\
\cline { 2 - 5 } & Variable Delay Block & FUS/8 & Offset_n & Total & \\
\hline \hline 1 & 250 & 450 & -104 & 596 & 59.6 \\
\hline 2 & 250 & 450 & -264 & 436 & 43.6 \\
\hline 3 & 250 & 450 & -228 & 472 & 47.2 \\
\hline 4 & 250 & 450 & -404 & 296 & 29.6 \\
\hline \hline \multicolumn{4}{|c|}{ Total Delay/Phase for One Revolution } & 1800 & 180.0 \\
\hline
\end{tabular}

The frequency of oscillation, $f_{0}$, can be calculated from the total delay of one 
revolution, Table 3.1, of the RTWO as follows:

$$
f_{0}=1 /(2 \times 1800 \times T S)=24.0 \mathrm{MHz}
$$

With this starting point the simulation ran for $23.5 \mu \mathrm{s}$, which included a $3.5 \mu \mathrm{s}$ initialization period and 10 correction iterations of $2.0 \mu \mathrm{s}$ each. The total delay for each TL section after the simulation was completed is shown in Table 3.2. We see that the variable delay block delays were adjusted to make the totals approximately equal, see right hand side of Figure 3.5 signals Dly1o to Dly4o. Therefore, the phases have been adjusted to be equal.

Table 3.2: Transmission Line Phase Delay at Simulation End

\begin{tabular}{|c|c|c|c|c|c|}
\hline \multirow{2}{*}{ TL Section } & \multicolumn{4}{|c|}{ Final 45 ${ }^{\circ}$ TL Section (TS) } & \multirow{2}{*}{$\Delta$ Phase ( ${ }^{\circ}$ ) } \\
\cline { 2 - 5 } & Variable Delay Block & FUS/8 & Offset_n & Total & \\
\hline \hline 1 & 104.4 & 450 & -104 & 450.4 & 45.015 \\
\hline 2 & 264.5 & 450 & -264 & 450.5 & 45.025 \\
\hline 3 & 228.1 & 450 & -228 & 450.1 & 44.985 \\
\hline 4 & 404 & 450 & -404 & 450.0 & 44.975 \\
\hline \hline \multicolumn{4}{|c|}{ Total Delay/Phase for One Revolution } & 1801 & 180.00 \\
\hline
\end{tabular}

The total delay for one revolution is approximately the same as it was at initialization; therefore, using equation (3.7) $f_{0}=23.987 \mathrm{MHz}$ at simulation end and has been constant throughout phase calibration. This is because the phase adjustment algorithm adjusts the largest and smallest phase delays errors by the same amount during each of its iterations. Figure 3.7 shows the FFT spectrum of the rail-to-rail signal, $\phi 8$ of Figure 3.2, to be similar to this calculated value, approximately $23.90 \mathrm{MHz}$. This simulated FFT frequency value did not change throughout the simulation. 


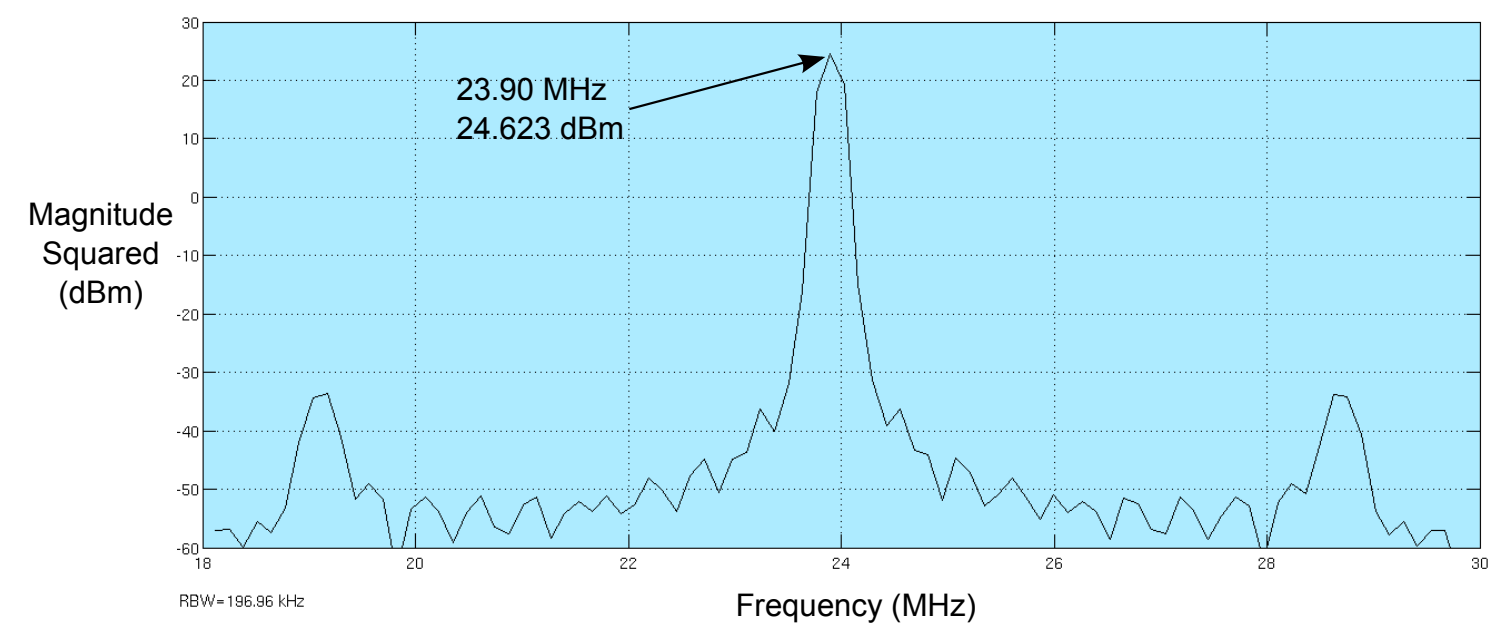

Figure 3.7: Simulink ${ }^{\circledR}$ FFT at RTWO Output (Point A)

Additional sub-blocks of the RTWO $24 \mathrm{MHz}$ Block are described in Appendix A.

\subsubsection{The Phase Alignment Algorithm}

The Phase Alignment Algorithm and its associated blocks are illustrated by Figure

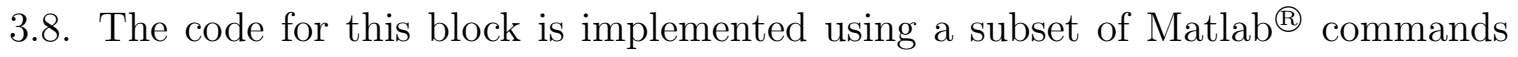
and has been included in Appendix B.3. The two timer blocks have been explained previously. The Stop output indicates when the algorithm has adjusted the RTWO phases to be within $45^{\circ} \pm$ Limit and has halted. The In1 to In4 signals of the Phase Alignment Algorithm block are the digitized phase measurements and the Dly1/4o outputs are the resulting RTWO delay adjustments (i.e., Point E of Figure 3.1 corresponds to Point E of Figure 3.8). The remaining input/output signals connect to a series of single TS delays contained in the Integer Delay Store block. This implements storage so the algorithm can base its current state on data from its previous state. The Sampling Rate Conversion block contains four Rate Transition blocks to convert the continuous signals from the algorithm block to discrete signals required by the Variable Integer Delay blocks shown in Figure A.2. 


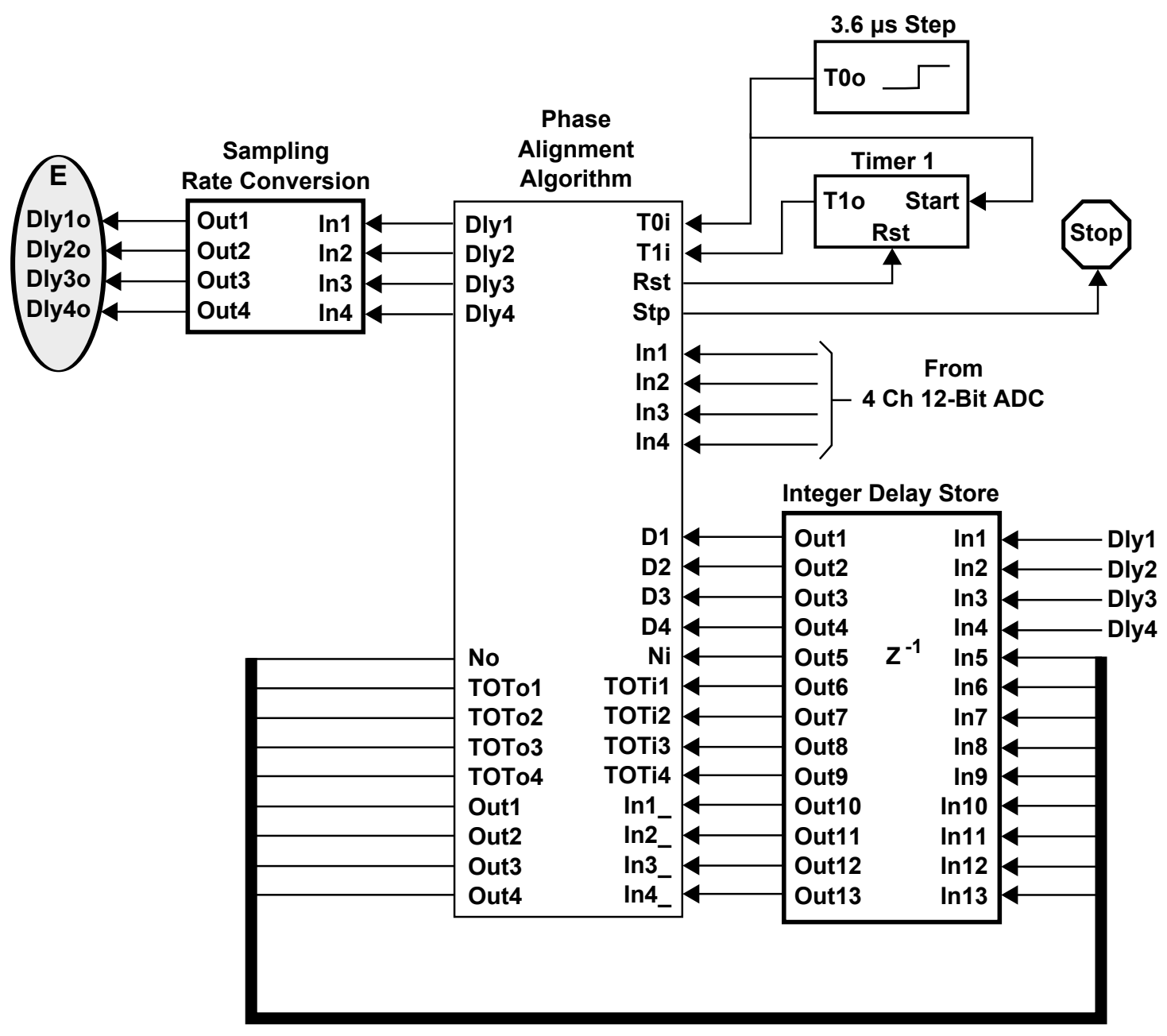

Figure 3.8: Simulink $^{\circledR}$ Algorithm Blocks

Figure 3.9 is a flow chart representation of the Matlab ${ }^{\circledR} /$ Simulink $^{\circledR}$ Phase Alignment Algorithm. During time T0 (i.e., initial $3.6 \mu s$ of simulation) the RTWO variables are initialized, the RTWO is started and allowed to reach steady-state. After T0 expires, T1 generates a pulse every $2.0 \mu s$ until the simulation stops. On each pulse of $\mathrm{T} 1$ the ADC phase measurements are read for all four differential TL sections, and the minimum and maximum phase delays are found. If the maximum phase delay minus the minimum phase delay is greater than a predefined limit (i.e., seven has been arbitrarily selected, other values will also work), then the TL delay skew is too 
large and must be corrected. Otherwise, stop.

Next, the delay adjustment step size is determined (i.e., Step $=$ Limit $\times 0.05$ ), which will be added to the minimum delay TL section and subtracted from the maximum delay TL section. The size of this step must be small enough so limit cycles will not occur that will prevent the algorithm stop condition from being met. The condition of multiple maximum and minimum TL sections is handled by dividing the step size by the number of equal length maximum and minimum TL sections to give Max_step and Min_step. Finally, the new variable delay values for the TL sections requiring adjustment are determined by adding Max_step and Min_step as required. After these new delays are programmed into the RTWO, the RTWO reaches a new steady-state after a settling time of $<13 \mu s$. This procedure is repeated on the next rising edge of timer $\mathrm{T} 1$. 


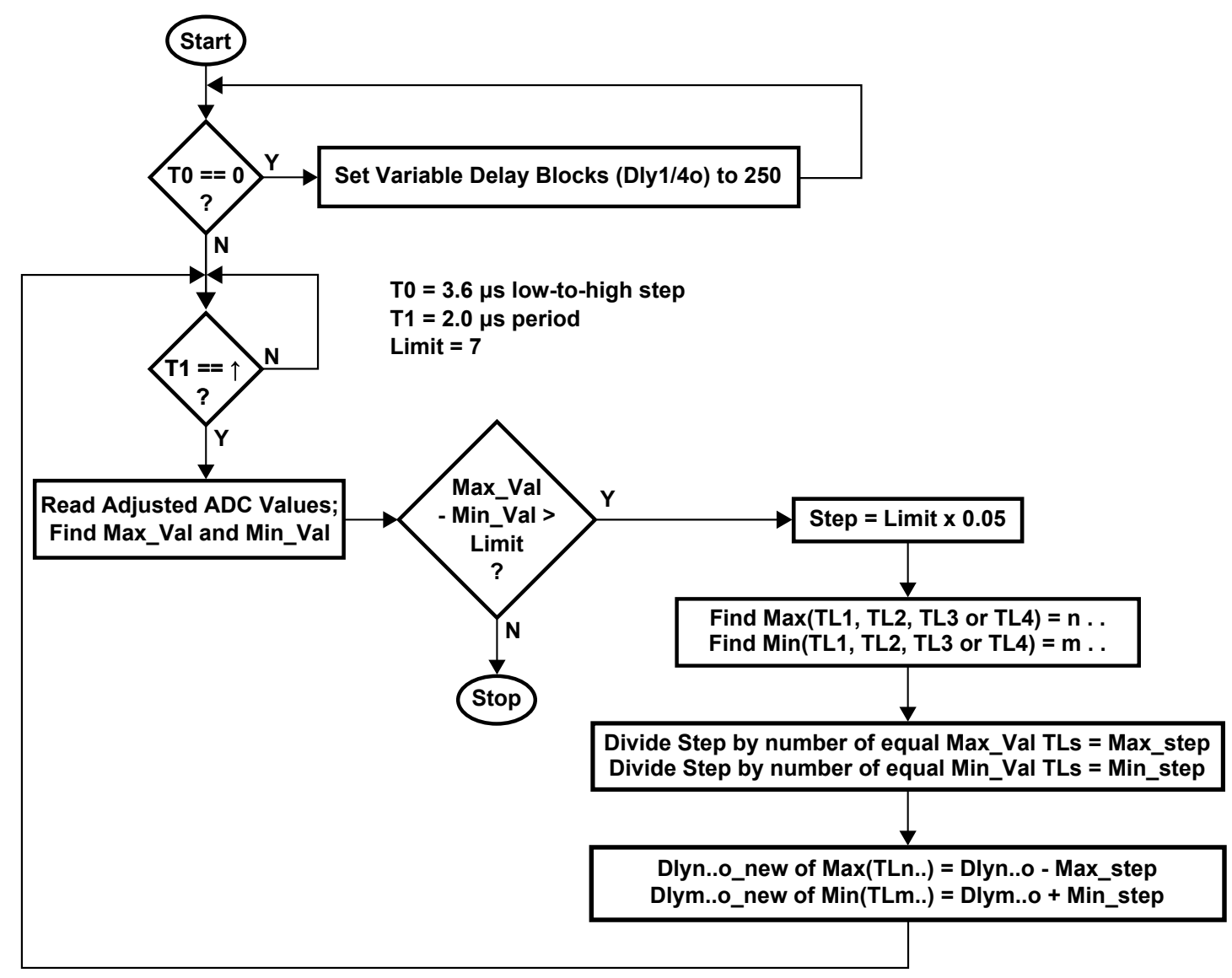

Figure 3.9: Simulink ${ }^{\circledR}$ RTWO Phase Alignment Algorithm Flow Chart

\subsection{Proof of Concept - Cadence ${ }^{\circledR}$ Virtuoso ${ }^{\circledR}$ AMS}

\section{Designer}

Figure 3.10 is a functional block diagram of the system used to demonstrate the concept of RTWO phase tuning in the Cadence ${ }^{\circledR}$ Virtuoso ${ }^{\circledR}$ AMS Designer mixed signal circuit simulator environment. 


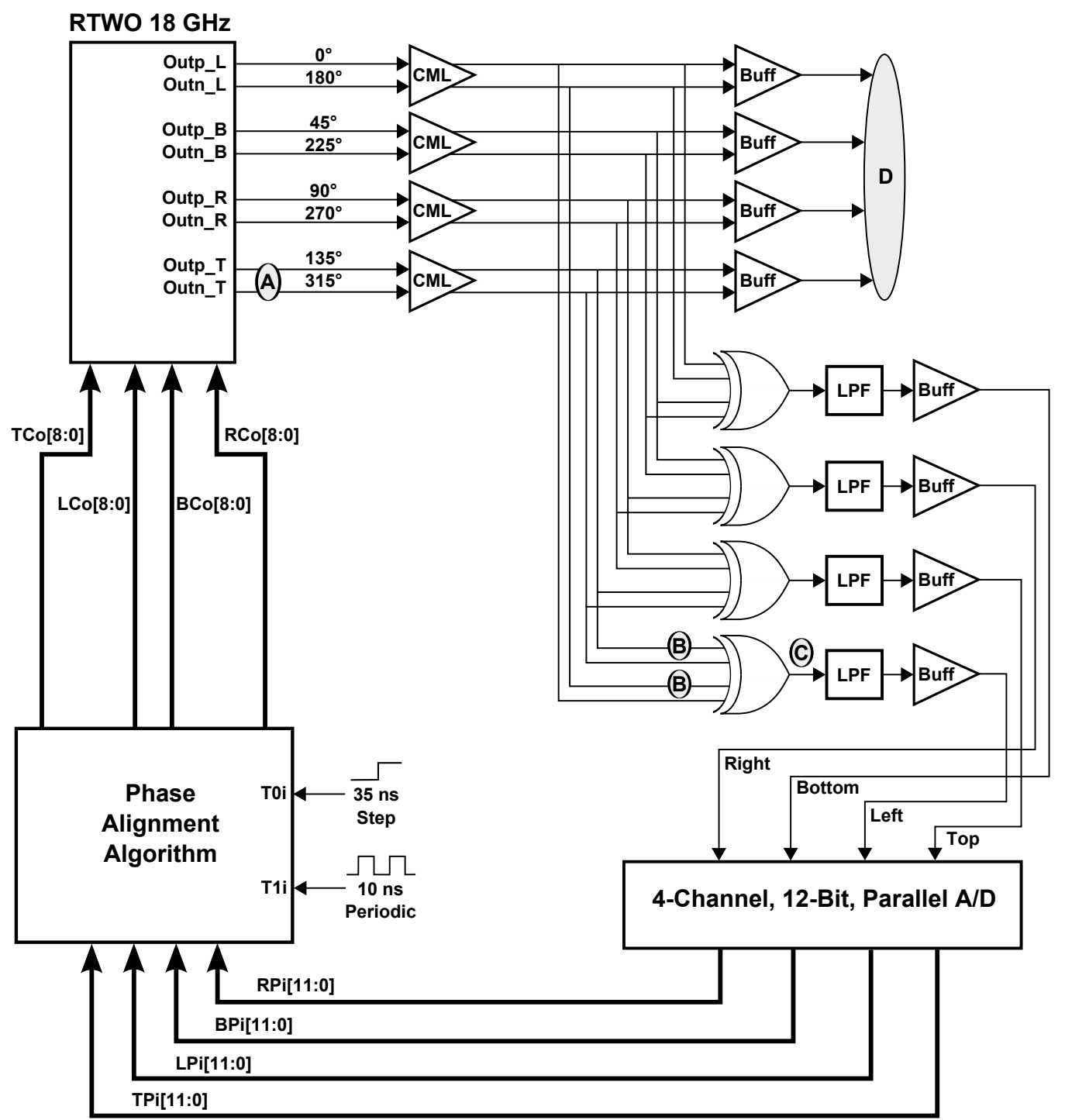

Figure 3.10: Cadence ${ }^{\circledR}$ Virtuoso ${ }^{\circledR}$ AMS Designer System Level RTWO Phase Calibration Circuit

The RTWO $18 \mathrm{GHz}$ block can be described as an implementation of Figure 1.1, where nodes $0^{\circ} / 180^{\circ}$ are in the bottom left corner; nodes $45^{\circ} / 225^{\circ}$ are in the bottom right corner; nodes $90^{\circ} / 270^{\circ}$ are in the top left corner and nodes $135^{\circ} / 315^{\circ}$ are in the top left corner of the structure. Similarly, for an anticlockwise travelling wave Outp/n_L is the end node of the left side; Outp/n_B is the end node of the bottom; Outp/n_R is the end node of the right side and Outp/n_t is the end node of the top 
of the structure.

The TLs of the RTWO $18 \mathrm{GHz}$ block have been implemented in several ways: initially, using a $130 \mathrm{~nm}$ CMOS kit microstrip TL models; then as 2.5D s-parameter data, generated from a TL layout using ADS-Momentum, in an NPORT block; and finally using ideal lumped R, L and C elements derived from ADS-Momentum simulation as described in [6]. The $130 \mathrm{~nm}$ CMOS kit TL models worked well; however, these models were not available in the NXP BiCOMS kit. Using the NXP BiCMOS kit, the NPORT s-parameter block using spline interpolation gave mixed results in a transient simulation. That is, after minor circuit changes the transient simulation would often function for a short period and then become unstable. The ideal lumped RLC RTWO implementation was more stable and ran more quickly, so it was used in many of circuit simulations that follow.

The Phase Alignment Algorithm block of Figure 3.10 is implemented in Verilog and is described in detail in section 3.3.3. The timer input T0i holds off the Phase Alignment Algorithm function for the first 35.0 ns of simulation while the initial RTWO phase error is set, the RTWO starts and reaches stead-state oscillation. After T0i is high, each rising edge of the $10 \mathrm{~ns}$ periodic clock signal at T1i initiates an iteration of the Phase Alignment Algorithm. Both these clock signals oscillate between 0 to $1.2 \mathrm{~V}$ and are generated from independent sources available in the simulator analogue library.

Figure 3.11 shows a sample of the differential output clock signals (Outp_T and Outn_T) at Point A, Figure 3.10, of the RTWO after it has reached steady-state oscillation. 


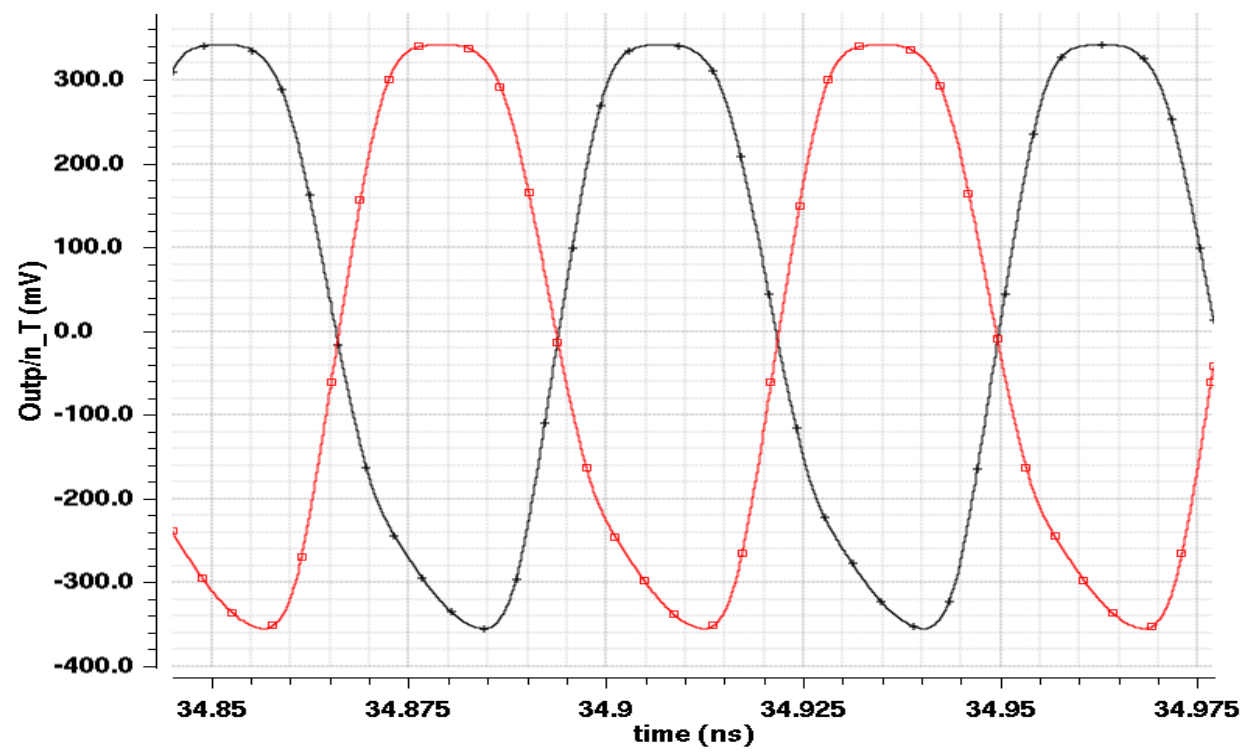

Figure 3.11: RTWO Differential Output Clock Signals (Point A)

These $\pm 300 m V$ differential clock signals are then converted to differential CML voltage levels (i.e., $0.85 V \pm 0.350 \mathrm{mV}$ ) using CML buffers implemented in Verilog-A. This conversion to CML was chosen to support signals operating at a frequencies in the order of $18 \mathrm{GHz}$. The CML clock signals are fed to differential to single ended buffers (Buff) and to CML XOR gates. The Buff block outputs, Point D, show the clock phases with phase skew intentionally programmed in the RTWO as a starting point for the phase alignment simulation, labelled Before Calibration in Figure 3.12. Specifically, the phases marked $\Delta 1=48.08^{\circ}$ (Top), between Outp_T/Outn_T and Outp_R/Outn_R, $\Delta 2=39.19^{\circ}$ (Right), between Outp_R/Outn_R and Outp_B/Outn_B, $\Delta 3=42.11^{\circ}$ (Bottom), between Outp_B/Outn_B and Outp_L/Outn_L, and $\Delta 4=50.61^{\circ}$ (Left), between Outp_L/Outn_L and Outn_T/Outp_T. It should be noted that these four phase deltas will always sum to $180^{\circ}$ in an RTWO that has reached steady-state travelling wave oscillation. The signals of Figure 3.12, labelled After Calibration, show all these phase differences equal to $45^{\circ} \pm$ Limit, where Limit is a predefined resolution, see 
RTWO Phase Alignment Flow Chart Figure 3.17. Here the calibration algorithm has iteratively adjusted the phase deltas, determined that the phase error between all the measured signals is within a predefined resolution and has stopped the algorithm as no further adjustment was required.
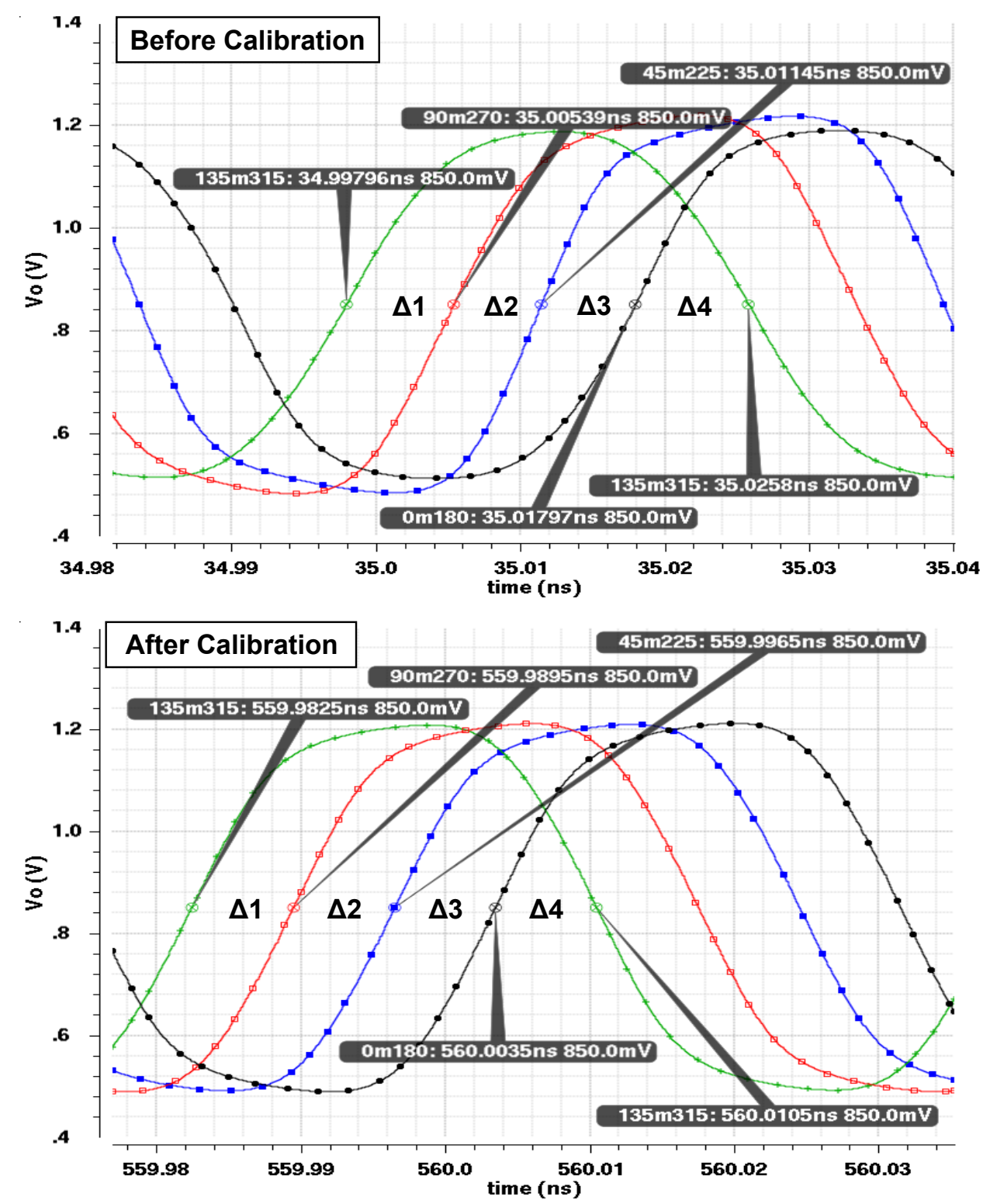

Figure 3.12: Output Phases (Point D) Before Calibraton - Top; After Calibration - Bottom

The labelled signals of Figure 3.12 show that the RTWO travelling wave front 
rotation is clockwise, i.e., $135 \mathrm{~m} 315$ precedes $90 \mathrm{~m} 270$, which precedes $45 \mathrm{~m} 225$, which precedes $0 \mathrm{~m} 180$. The steady-state frequency of oscillation is approximately $17.95 \mathrm{GHz}$ before calibration and approximately $17.86 \mathrm{GHz}$ after calibration.

\subsubsection{RTWO Phase Measurement}

In Figure 3.10 the differential clock signals representing the relative phases of the four differential TL sections of the RTWO are applied consecutively to the inputs of four XOR phase detectors implemented in Verilog-A. The XOR gates produce a duty cycle signal that is proportional to the phase taken up by each differential TL section. In Figure 3.13 we can see that the phase difference between input signals is represented by the duty cycle of the output signal.

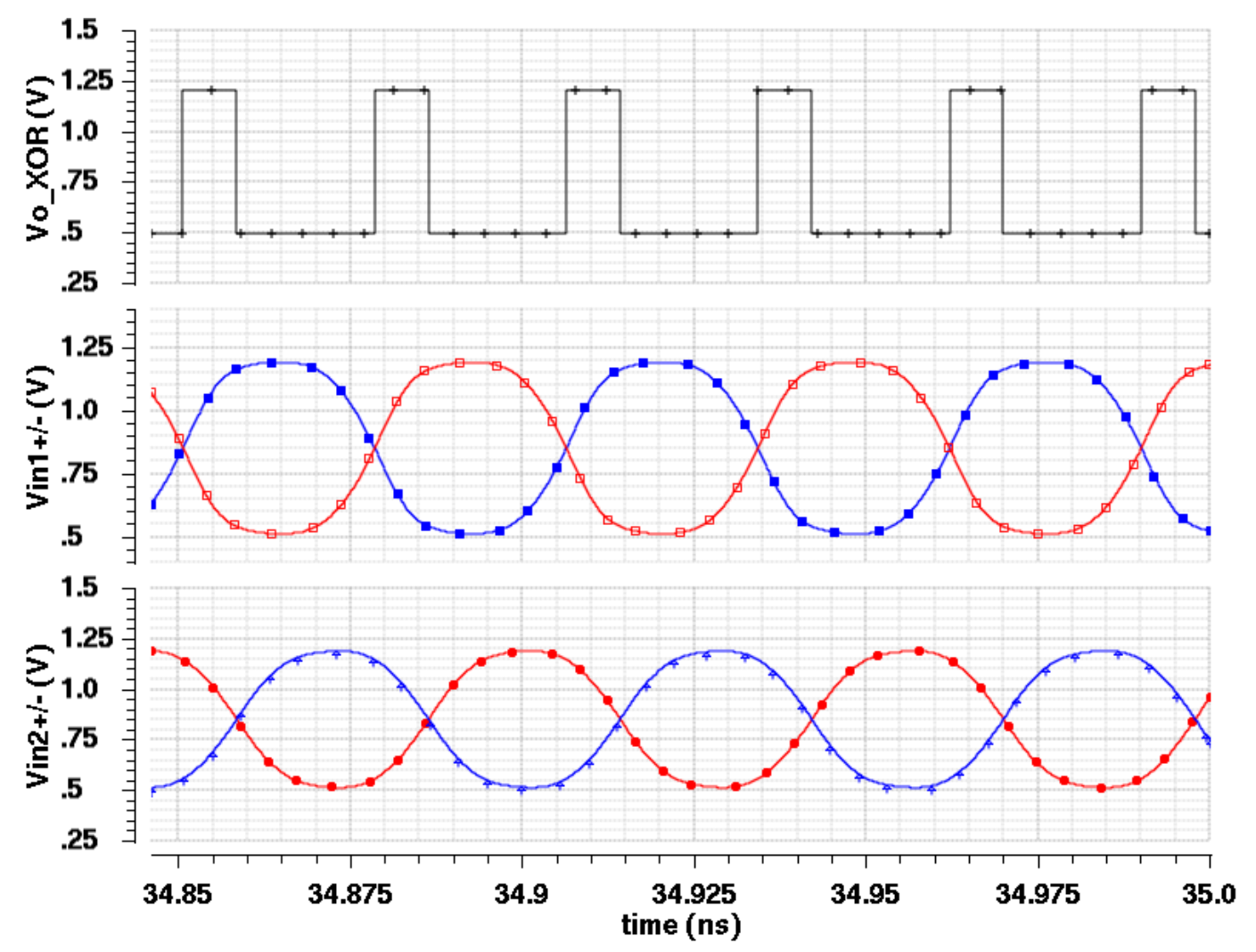

Figure 3.13: CML XOR - Phase in to Duty Cycle Out (Points B \& C) 
It should be noted that the phase of the differential inputs feeding the bottom XOR from the top CML gate (i.e., Outp_L and Outn_L) is reversed so the measurement will be over the single remaining TL section rather than the three TL sections already measured. In fact, this fourth XOR phase detector is redundant as this phase difference at steady-state can be determined by subtracting the sum of the other three measured phases from $180^{\circ}$, see equation (3.1).

The outputs of these XOR phase detectors are single-ended CML (i.e., $0.85 \mathrm{~V} \pm$ $0.350 \mathrm{mV}$ ) clock signals of twice the frequency of the input signals with output duty cycles proportional to the input phase differences. These duty cycles are averaged through LPF blocks, which consists of two cascaded single order LPFs with cut-off frequencies of $79.6 \mathrm{MHz}$ followed by $318.3 \mathrm{MHz}$. Next the single-ended input to single-ended output Buff block, implemented in Verilog-A, is used to provide gain and bias control. Here the gain of this block is set to one with a bias of $-0.5 \mathrm{~V}$ to remove the $0.5 \mathrm{~V}$ offset shown in the top plot of Figure 3.13, which passes directly through the LPF. The amplitudes of these signals (i.e., Right, Bottom, Left and top on Figure 3.10) are proportional to the measured phases at each iteration of the phase alignment algorithm.

Next these signals are converted to binary numbers using an ideal 4-Channel 12Bit Analogue-to-Digital Converter (ADC) implemented in Verilog-A. These numbers are passed over 12-bit buses $\mathrm{RPi}[11: 0], \operatorname{RPi}[11: 0], \operatorname{RPi}[11: 0]$ and $\mathrm{RPi}[11: 0]$ to the Phase Alignment Algorithm block, which finds the TL section that has the largest and the TL section has the smallest phase measurement. It then uses this information to adjust variable capacitance shown in Figure 3.16, through 9-bit buses TCo[8:0], $\mathrm{LCo}[8: 0], \mathrm{BCo}[8: 0]$ and $\mathrm{RCo}[8: 0]$, which control the phase of each differential TL section of the RTWO. These functions are triggered every 10 ns by the periodic clock signal at T1i. This cycle time is set to be the same as the settling time of the 
RTWO after a change in phase delay. Figure 3.14 illustrates these signals at Right, Bottom, Left and Top of Figure 3.10 for each cycle of T1i (i.e., iteration of the Phase Alignment Algorithm). In this figure $175 \mathrm{mV}$ represents $45^{\circ}$. At time $=35 \mathrm{~ns}$ the initial phase error, programmed into the RTWO by the Phase Alignment Algorithm (see Appendix B.1) is marked; at time $=560$ ns the final phase error is marked. After time $=560$ ns the Phase Alignment Algorithm ceases to make further phase corrections as the Limit requirement has been met.

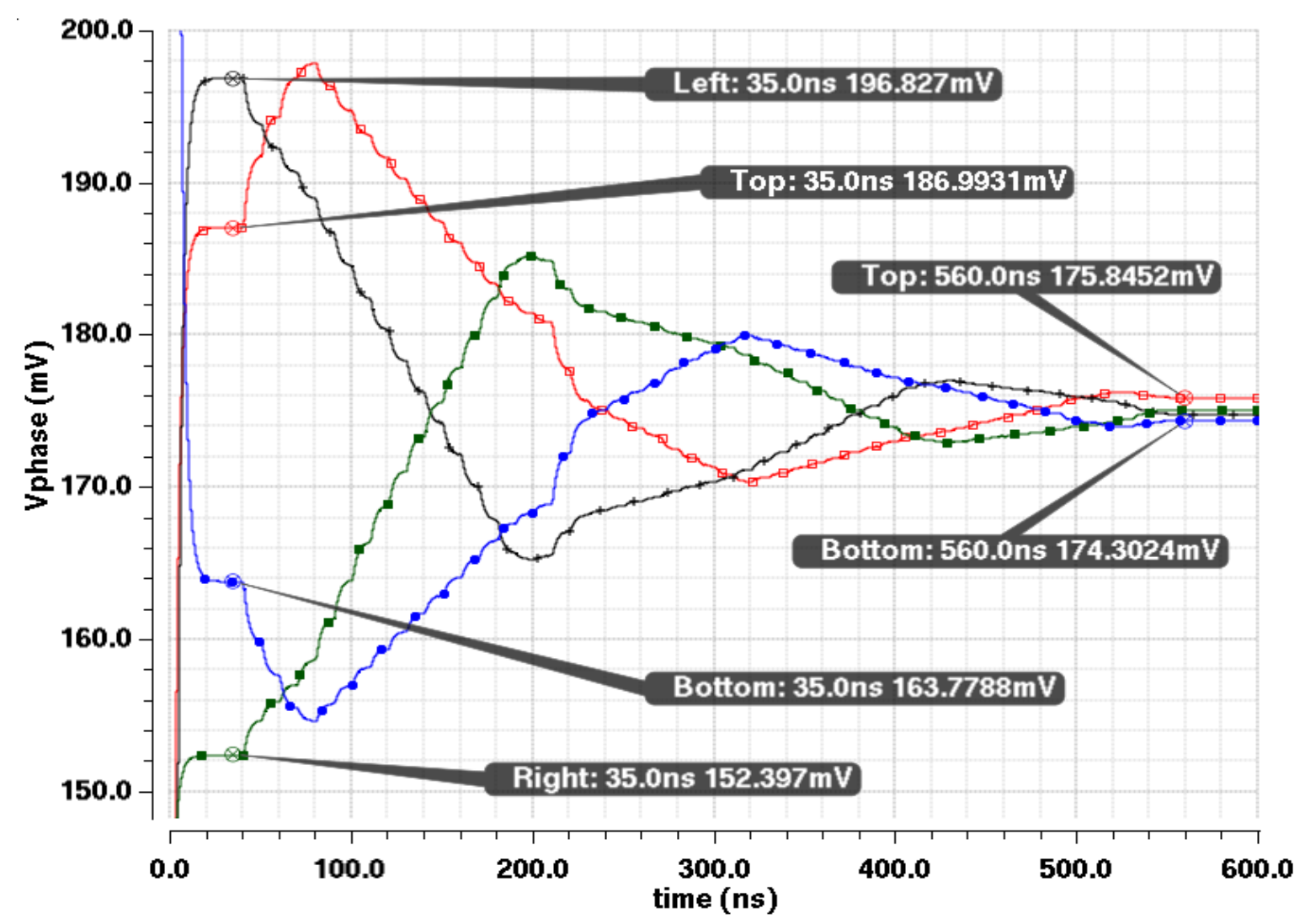

Figure 3.14: Phase Measurement Output Voltages

The Buff blocks of Figure 3.10 have a maximum output signal of $0.7 \mathrm{~V}$ and the ADC reference voltage is $1.2 \mathrm{~V}$, which results in the following measurement resolution:

$$
(0.7 / 1.2)\left(2^{12}-1\right)=2388.75 \text { bits }
$$




$$
\begin{gathered}
0.7 \mathrm{~V} / 2388.75 \text { bits }=293.0 \mu \mathrm{V} / \mathrm{bit} \\
180^{\circ} / 2388.75 \text { bits }=75.35 \mathrm{~m}^{\circ} / \mathrm{bit} \\
293.0 \mu \mathrm{V} / \mathrm{bit} / 75.35 \mathrm{~m}^{\circ} / \mathrm{bit}=3 . \overline{8} \mathrm{mV}{ }^{\circ}
\end{gathered}
$$

This result can be stated more simply from the full-scale voltage and full-scale phase as follows:

$$
0.7 \mathrm{~V} / 180^{\circ}=3 . \overline{8} \mathrm{mV} /{ }^{\circ}
$$

The algorithm stop resolution or Limit was set to six for the above simulation to give $6 \times 75.35 \mathrm{~m}^{\circ} / \mathrm{bit}=0.452^{\circ}$ i.e., Limit $=0.452^{\circ}$. Table 3.3 shows the marked points of Figure 3.14 in degrees, i.e., by dividing the marker voltage by the result of equation (3.12).

Table 3.3: Phase Alignment Simulaiton Results

\begin{tabular}{|c|c|c|}
\hline TL Section & Before Calibration (35.0 ns) & After Calibration (560.0 ns) \\
\hline \hline Left & $50.61^{\circ}$ & $\geq 44.826^{\circ} / \leq 45.217^{\circ}$ \\
\hline top & $48.08^{\circ}$ & $45.217^{\circ}$ \\
\hline Bottom & $42.11^{\circ}$ & $44.826^{\circ}$ \\
\hline Right & $39.19^{\circ}$ & $\geq 44.826^{\circ} / \leq 45.217^{\circ}$ \\
\hline
\end{tabular}

Here we can subtract Bottom from Top to get the final phase error, i.e., $45.217^{\circ}-$ $44.826^{\circ}=0.391^{\circ}$, which is less than the Limit $=0.452^{\circ}$.

\subsubsection{The Virtuoso ${ }^{\circledR}$ AMS Designer RTWO Block}

The block RTWO $18 \mathrm{GHz}$ of Figure 3.10 contains an RTWO implemented using the ideal lumped RLC elements of Figure 3.15, which are divided into eight $45^{\circ}$ TL sections tapped to provide differential outputs at ports P1/P2, P3/P4, P5/P6 
and P7/P8. The back-to-back inverters, marked -Gm, provide oscillator gain, and the output buffers provide isolation between the oscillator and load of any external circuitry. These gain elements were implemented using the NXP BiCMOS kit by K.T. Ansari of [6] and have been use here without modification or analysis.
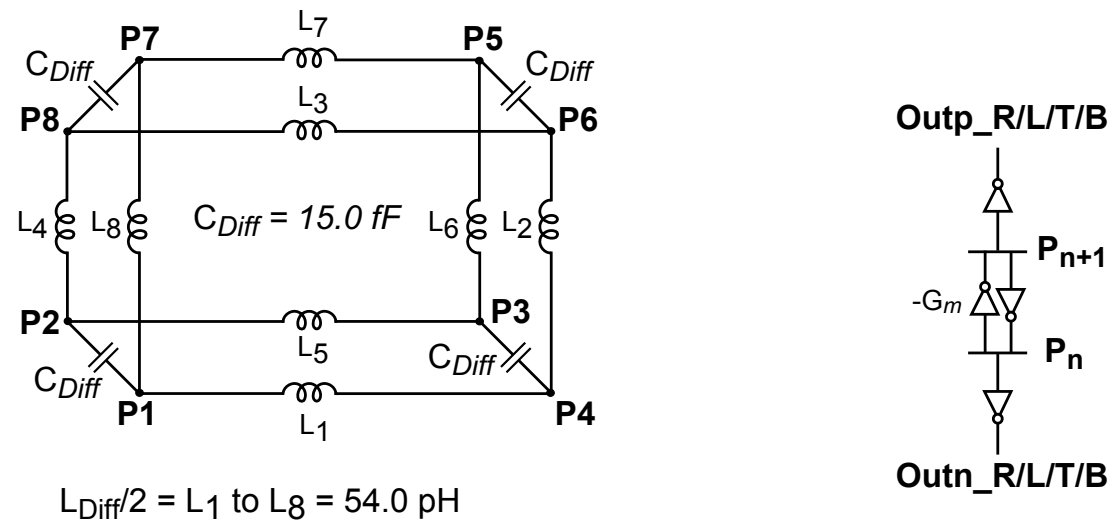

Series $\mathrm{R}=50 \mathrm{mOhm}$ included in every Inductor $\mathrm{L}$.

Figure 3.15: Lumped Element RTWO based on Equations from $[6,7]$

The digital control outputs $\mathrm{TCo}[8: 0], \mathrm{LCo}[8: 0], \mathrm{BCo}[8: 0]$ and $\mathrm{RCo}[8: 0]$ from the Phase Alignment Algorithm tune the variable capacitance of RTWO TL sections Top, Left, Bottom and Right, see Figure 3.10. Each 9-bit bus is connected to the [C256:C1] control signals shown in Figure 3.16 (i.e., C256 to bus signal 8 .. C1 to bus signal $0)$. Here $C=500 a F$ was determined through simulation.

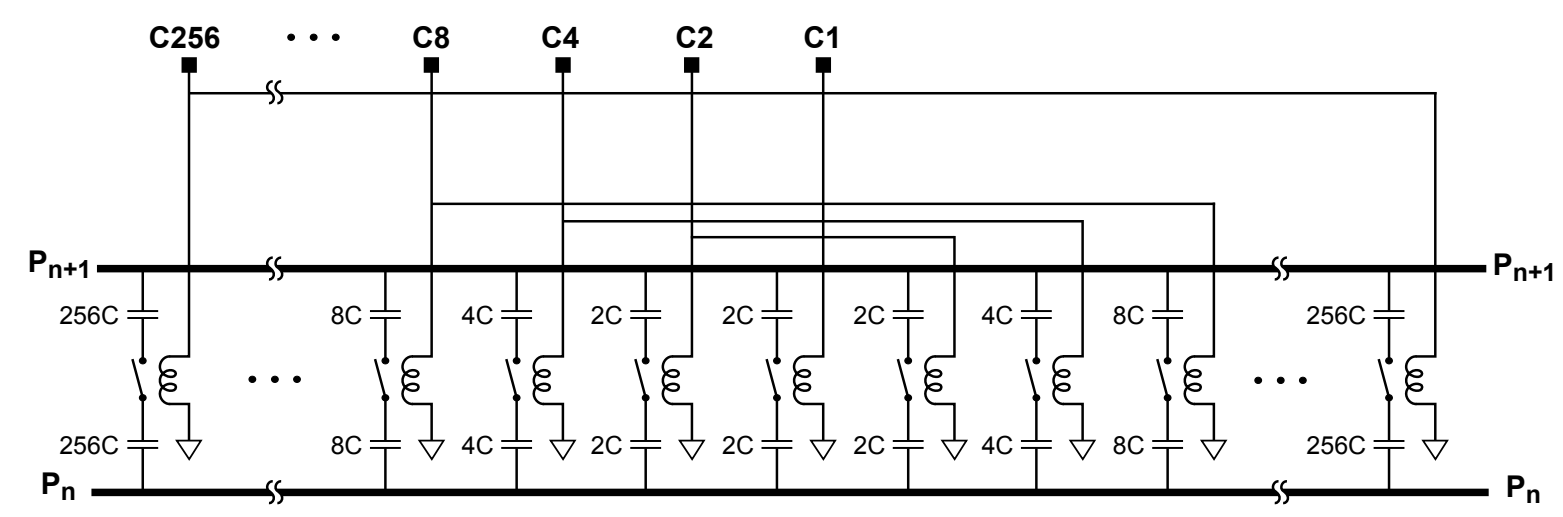

Figure 3.16: Digitally Controlled Ideal Capacitor Tuning Array 
An updated version of [6] provides the RLC values that were used as a starting point for the RTWO in this simulation. These values were determined by 2.5D ADSMomentum simulations performed on the RTWO TLs. In this paper equation (3.13), a refinement of equation (2.2), was used to determine the operating frequency $\left(f_{o}\right)$ of an RTWO with four TL sections, i.e., $n=4$.

$$
f_{0}=\frac{1}{2 l_{\text {total }} \sqrt{L_{\text {perlen }}\left(C_{o}+\frac{C_{\text {seg }}}{l s e g}\right)}}
$$

where, $l_{\text {total }}=1.488 \mathrm{~mm}$ is the total physical length of the loop, $L_{\text {perlen }}=$ $423 \mathrm{nH} / \mathrm{m}$ is the per unit length differential inductance of the loop, $C_{o} / n=$ $101 \mathrm{pF} / \mathrm{m} / 4=25.25 \mathrm{pF} / \mathrm{m}$ is the per unit length differential capacitance of the interconnect, $C_{\text {seg }}=254.55 \mathrm{fF}$ is the total differential capacitance of each TL segment and $l_{\text {seg }}=l_{\text {total }} / n=1.488 \mathrm{~mm} / 4=372 \mu \mathrm{m}$ is the length of each TL segment. This gives:

$$
\begin{aligned}
f_{0} & =\frac{1}{2\left(1.488 \times 10^{-3}\right) \sqrt{423 \times 10^{-9}\left(25.25 \times 10^{-12}+\frac{254.55 \times 10^{-15}}{372 \times 10^{-6}}\right)}} \\
& =19.40 \mathrm{GHz}
\end{aligned}
$$

which is consistent with [6]. This frequency was reduced to approximately $18 \mathrm{GHz}$ after additional parasitics were included in the implemented design.

$C_{\text {seg }}$ of equation (3.13) can be described as follows:

$$
C_{\text {seg }}=C_{V a r}+C_{-G m}+C_{b u f f}
$$

where, for each TL section $C_{V a r}$ is the variable tuning capacitance; $C_{-G m}$ is the 
total capacitance associated with the back-to-back gain elements; and $C_{b u f f}$ is the capacitance of the signal tapping buffers. In the system simulation of Figure 3.10 $C_{V a r}$ was set to a nominal value and the additional capacitor tuning array of Figure 3.16 was used for phase tuning. The starting capacitance of this array was $256 \times C=$ $256 \times 500 a F=128 \mathrm{fF}$. The nominal value of $C_{V a r}=46 \mathrm{fF}$; therefore, the RLC components were adjusted through simulation to compensate for this change. The simulated values are: $R=50 m \Omega$ (starting value $1977 \Omega / m \times l_{\text {total }} / 2 n=367.7 m \Omega$ ); $C_{\text {Diff }}=15.0 \mathrm{fF}$ (starting value $\left.C_{o} \times l_{\text {total }} / 2 n=18.786 \mathrm{fF}\right)$ and $L_{D i f f / 2}=54.0 \mathrm{pH}$ (starting value Lperlen $\times l_{\text {total }} / 2 n=78.678 \mathrm{pH}$ ) to produce an operating frequency of approximately $17.9 \mathrm{GHz}$. It should be noted that values of $R \geq 80 \mathrm{~m} \Omega$ caused the AMSD mixed signal simulation to become unstable.

\subsubsection{The Phase Alignment Algorithm}

Figure 3.17 is a flow chart representation of the Cadence ${ }^{\circledR}$ Virtusos ${ }^{\circledR}$ AMS Designer Phase Alignment Algorithm. When $T 0 i=0 \mathrm{~V}$ (i.e., initial 35 ns of simulation) the RTWO variables are initialized, the RTWO is started and allowed to reach steadystate. After $T 0 i=1.2 \mathrm{~V}$ the $\mathrm{T} 1 \mathrm{i}$ receives a rising edge every $10 \mathrm{~ns}$ until the simulation stops. On each rising edge of T1i the ADC phase measurements are read for all four differential TL sections, and the minimum and maximum phase delays are found. The maximum phase delay minus the minimum phase delay is compared to three limits to determine the size of the correction step that will be made to the tuning varactors voltages. If the $\max / \min$ phase delay difference is smaller than the smallest limit, then the algorithm will stop.

The size of the varactor tuning step must be small enough so limit cycles will not occur that will prevent the algorithm stop condition from being met. The TL sections with maximum and minimum phase delay are then determined and the tuning step is 
subtracted from the minimum (varactor voltage decreased) and added to maximum (varactor voltage increased) phase TL sections. After these new varactor settings are programmed into the RTWO, the RTWO reaches a new steady-state after a settling time of $<10$ ns. This procedure is repeated on the next rising edge of timer T1i.

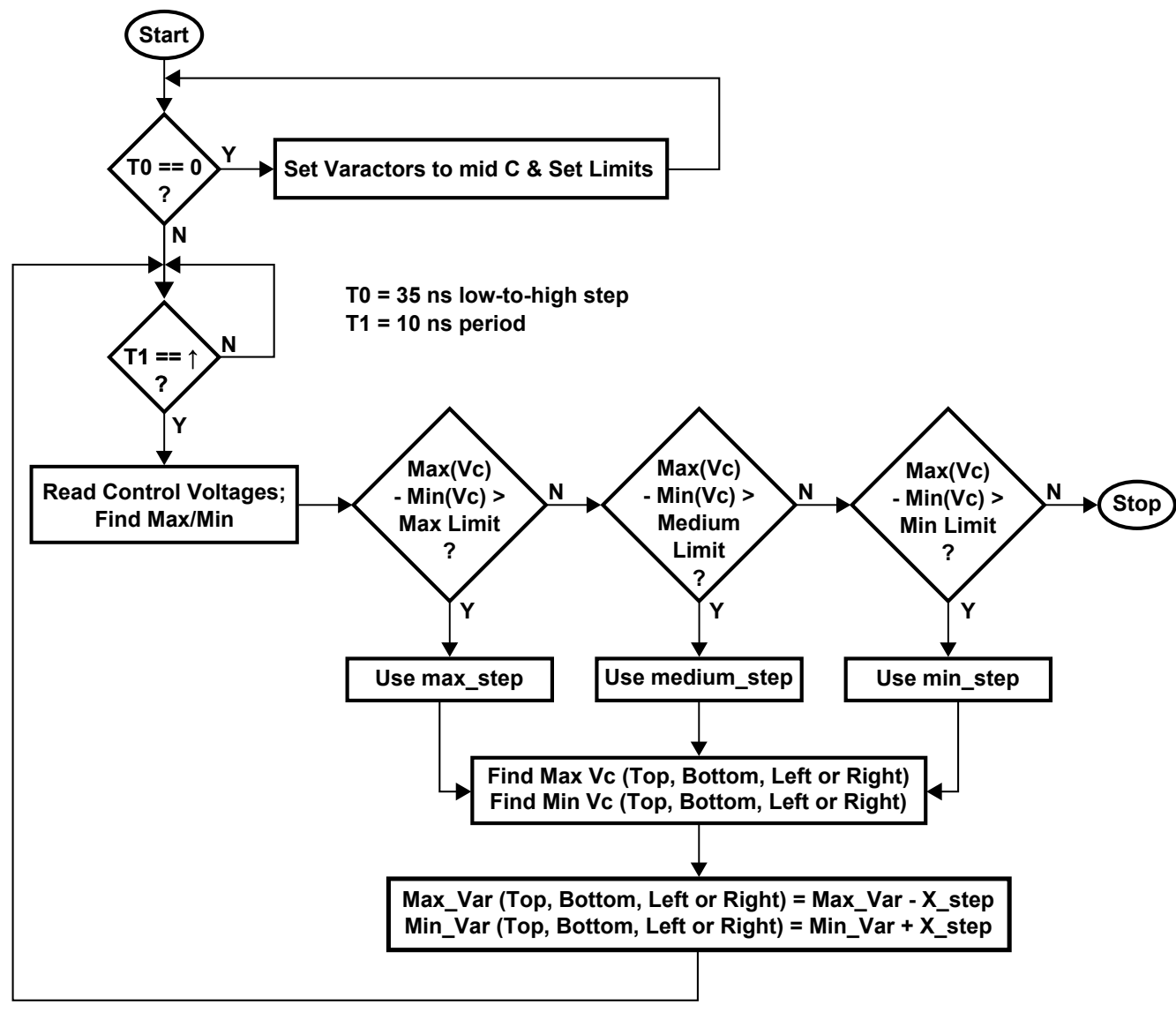

Figure 3.17: Cadence RTWO Phase Alignment Algorithm Flow Chart

The Verilog implementation of the flow chart of Figure 3.17 has been included in Appendix B.1. Using the result of equation (3.11) the Max Limit is $63 \times 75.35 \mathrm{~m}^{\circ} /$ bit $=2.7126^{\circ}$ and the Medium Limit is $15 \times 75.35 \mathrm{~m}^{\circ} /$ bit $=1.13025^{\circ}$, the Min Limit was previously calculated to be Limit $=0.452^{\circ}$. The capacitance array tuning steps are max_step $=11 \times 500 a F=5.5 \mathrm{fF}$, medium_step $=3 \times 500 a F=$ $1.5 \mathrm{fF}$, and min_step $=1 \times 500 a F=500 a F$. 


\section{Chapter 4}

\section{Phase Measurement Circuit}

\subsection{Phase Detector Selection}

An RTWO can be considered a distributed amplifier special case (i.e., output signals flipped $180^{\circ}$ and fed back to the input) where the drain and gate parasitic capacitances of the differential gain stages are absorbed into the RTWO transmission lines. This significantly raises the gain-bandwidth product of the gain stages, which allows the RTWO to have gain and oscillate at higher frequencies than would be expected from a conventional LC-oscillator of the same process. This is straight forward to simulate and has been realized in $130 \mathrm{~nm}$ CMOS at $18 \mathrm{GHz}$ and $36 \mathrm{GHz}$ in [19].

A Current Mode Logic (CML) XOR gate was selected as the best phase detector candidate based on its high speed operation [27], differential architecture and simplicity. That is, it has only two transistors in its switching (charging path) path; therefore, it will have fewer parasitic circuit elements and a greater potential for high speed operation when compared to more complicated implementations (i.e., sample and hold, flip-flop ...).

An additional advantage of an RTWO is that its structure is intrinsically differential. This advantage can be maintained in oscillator signal paths outside the 
RTWO by tapping its relative phases differentially. With this approach the maximum relative phase difference between any two differentially tapped signals along the RTWO is $180^{\circ}$. Therefore, a simple phase detector like an XOR (XNOR) with a gain of $K_{\text {phase }}= \pm V_{\text {range }} / \pi(\mathrm{V} / \mathrm{rad})[9]$ has an adequate measurement range for this application.

Figure 4.1 shows a differential XOR phase detector realized using CML. Tapped points along the RTWO are connected to the differential inputs $\mathrm{A}+/-$ and $\mathrm{B}+/-$ to produce outputs Out+/-, which are averaged through Low Pass Filters (LPF) to produce voltage outputs proportional to an input phase difference.

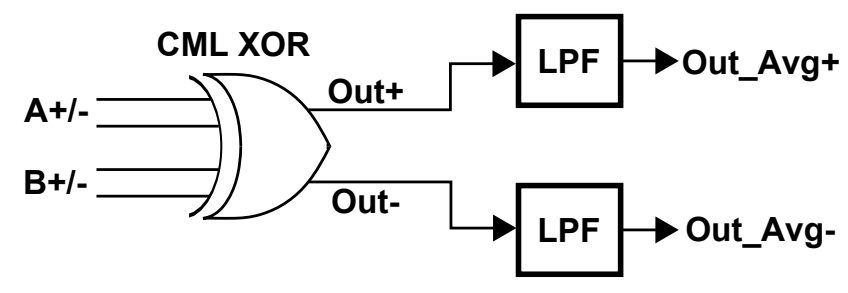

Figure 4.1: CML XOR Function with Averaged (LPF) Outputs

The XOR phase detector produces an output signal that is twice the input signal frequency with a duty cycle that is linearly proportional to and has a monotonic relationship with the phase difference between the two input signals through $180^{\circ}$.

Figure 4.2 illustrates the XOR phase detector transfer function after the dutycycle of the output signal is averaged through a LPF to produce a constant level signal between $V_{C C}$ and $V_{C C}-\Delta V$. Both outputs of Figure 4.2 are shown and the equations for average output voltages are as follows: 


$$
\begin{aligned}
V_{\text {Out_Avg }+} & =\left(V_{C C}-\Delta V\right)+K_{\text {phase }}(\phi A-\phi B) \\
& =\left(V_{C C}-\Delta V\right)+(\Delta V / \pi)(\phi A-\phi B) \\
V_{\text {Out_Avg- }} & =\left(V_{C C}-\Delta V\right)+(\Delta V / \pi)(\phi B-\phi A)
\end{aligned}
$$
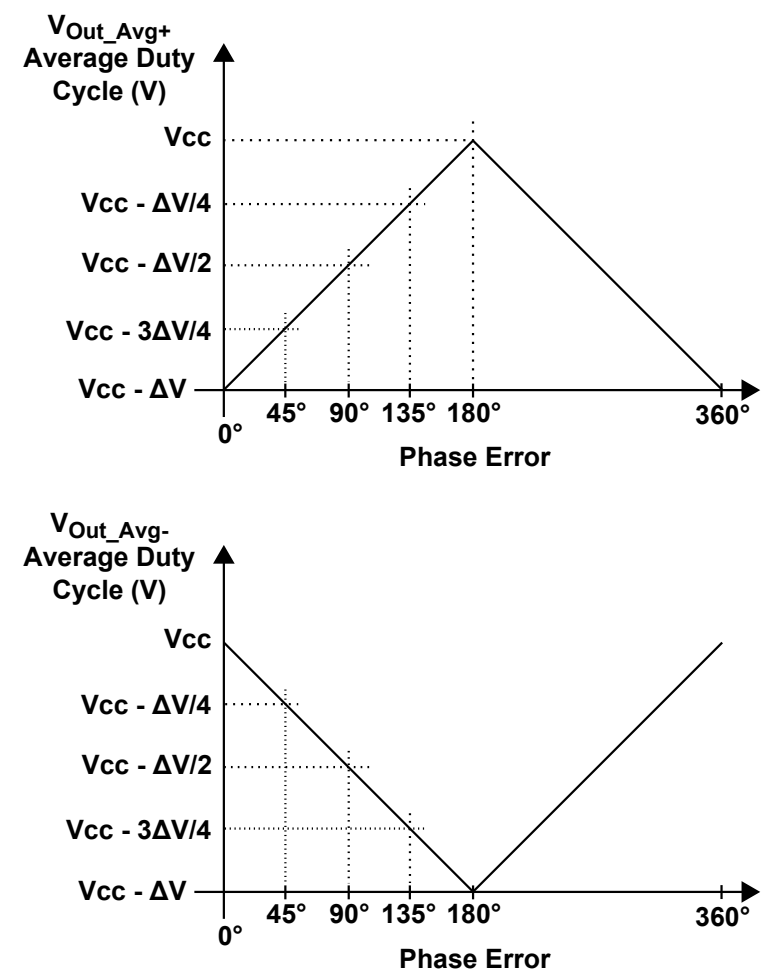

Figure 4.2: CML XOR Transfer Function Including LPF

\subsection{Circuit Implementation}

CML was chosen as the XOR structure for this application because of the differential signal and speed requirements. Initial simulations were done using a $130 \mathrm{~nm}$ NMOS 
differential transistor pair, but these transistors did not switch current completely on or completely off at microwave frequencies. The result was a non-linear transfer function that changed with frequency and would require a complex calibration scheme to linearize. This problem was mitigated by moving to a SiGe BiCMOS process. Fortunately, an $18 \mathrm{GHz}$ RTWO had been implemented previously in this process, so the objective was changed to phase tune this RTWO.

The following steps were used to design the XOR circuit:

1. Base Resistance $(\mathrm{R})$ and Capacitance $(\mathrm{C})$ have been identified as the major parasitic components limiting the switching speed of SiGe HBT device $[28,29]$. Next, in descending order of importance, is load RC, forward transit delay $\left(\tau_{F}\right)$, collector $\mathrm{RC}$ and finally emitter $\mathrm{RC}$. In order to mitigate these parasitics minimum size, high speed, high current transistors were selected for this circuit (i.e., width/length/emitters $=0.4 \mu \mathrm{m} / 1.3 \mu \mathrm{m} / 4$ ) [30].

2. Next a minimum size differential pair, implemented with these bipolar transistors, with resistive loads was verified to switch current on and off completely at more than $20 \mathrm{GHz}$.

3. Collector current $\left(I_{C}\right)$ was selected for high speed switching. This was done by selecting an $I_{C}$ that approaches maximum $f_{\mathrm{T}}$ (minimum $\tau_{F}$ from equation (4.7)) operation $[29,31,32]$. At maximum $f_{\mathrm{T}} I_{C}$ is approximately $18.0 \mathrm{~mA}$ and since $I_{E}=I_{C} / \alpha$ this current was selected as the bias or tail current for the differential transistor pairs.

4. The output voltage swing $(\Delta V)$ was selected next. $\Delta V_{\min }$ needs to be large enough to switch the differential pair on or off completely i.e., $>5 V_{T}=130 \mathrm{mV}$ at room temperature $[11,33] . \Delta V_{\max }$ should be low enough to not impact circuit 
speed (i.e., $\tau_{D} \propto R_{L} C_{E F F} \approx \Delta V / I_{C}$, so total gate delay is proportional to the voltage swing) and to allow easy biasing of stacked transistors between $V_{C C}$ and ground. $\Delta V_{\max }$ should be high enough so that the stacked differential transistor pairs of successive circuits will continue to switch current correctly over PVT. The range of $400 m V \leq V \max \leq 500 \mathrm{mV}$ was chosen to meet these requirements [32].

5. The CML load resistors were chosen to be $24 \Omega$. Q2 of Figure 4.3 will drive 18.0 $\mathrm{mA}$ through to the load resistors in the XOR topology, which gives $\Delta V=$ $18 m A \times 24 \Omega=0.432 \mathrm{~V}$ to meet the requirements of item 4 in this list.

\subsection{Removal of Unequal Transit Delays}

Consider two differential signals, tapped from an RTWO, that have a phase difference of $90^{\circ}$. One differential signal is applied to the $\mathrm{A}+/$ - inputs of the XOR gate of Figure 4.3 and the other differential signal is applied to the $\mathrm{B}+/$ - inputs.

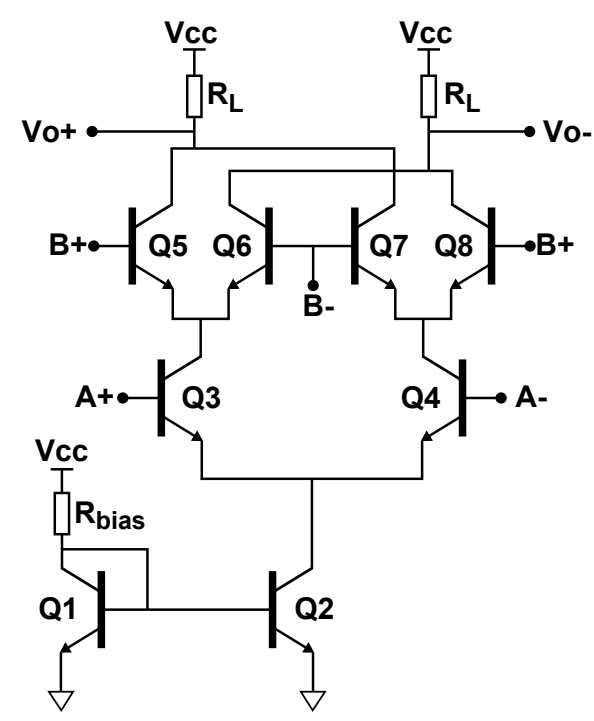

Figure 4.3: Bipolar CML XOR Schematic 
If we assume that these signals are biased correctly, the transistors are all the same size, and if we ignore any impedance difference between the A and B inputs, and ignore delay paths that are common, the A inputs to outputs $\mathrm{V} 0+/$ - path length $\left(\right.$ delay $\left.=\tau_{A}\right)$ is different from the B inputs to outputs V0+/- path length $\left(\right.$ delay $\left.=\tau_{B}\right)$ as follows:

$$
\begin{gathered}
\tau_{\Delta}=\tau_{A}-\tau_{B} \\
\tau_{A}=\tau_{B C}+\tau_{\text {Trace }}+\tau_{F} \\
\tau_{B}=\tau_{B C} \\
\tau_{\Delta}=\tau_{B C}+\tau_{\text {Trace }}+\tau_{F}-\tau_{B C} \\
=\tau_{\text {Trace }}+\tau_{F}
\end{gathered}
$$

where: $\tau_{\Delta}$ is the time difference between the two paths; $\tau_{B C}$ is the transit delay from transistor base to collector; $\tau_{\text {Trace }}$ is the path delay through the traces from the collectors of transistors Q3 and Q4 to the emitters of transistors Q5 to Q8; and $\tau_{F}$ is the forward transit delay from transistor emitter to collector.

From equation (4.6) we can see that the XOR gate will have an output duty cycle error of $\tau_{\Delta}$, which will result in an output offset error in a phase detector application. This error can be minimized by keeping $\tau_{\text {Trace }}$ very short and operating the transistors at a current density for maximum $f_{\mathrm{T}}[11]$, where:

$$
f_{\text {TMAX }} \approx 1 / 2 \pi \tau_{F}
$$

A more direct solution is to never introduce the problem in the first place by 
constructing an XOR gate that is absolutely symmetrical. The Symmetrical XOR (SXOR) gate [10] is illustrated in Figure 4.4.

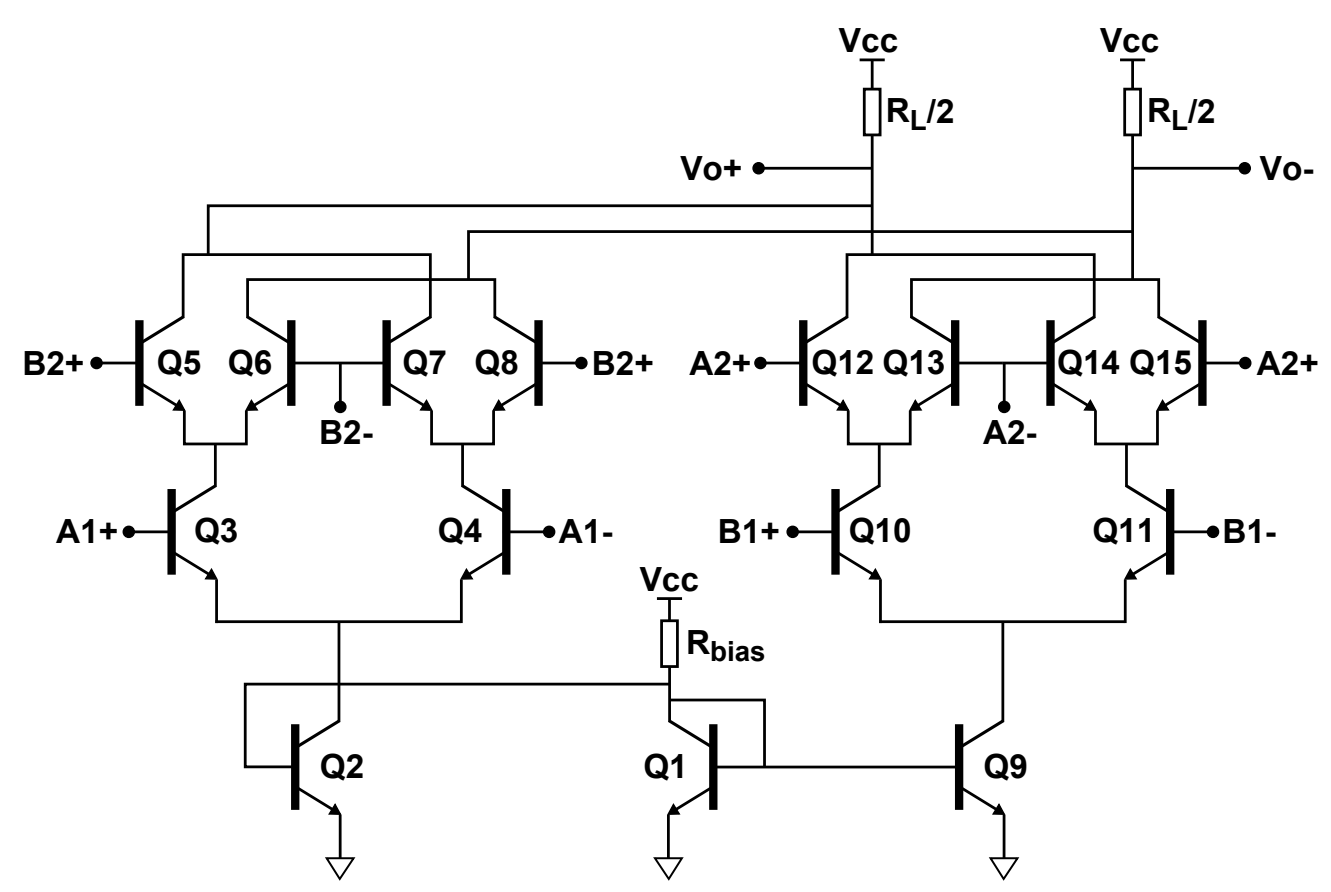

Figure 4.4: Bipolar CML SXOR Schematic

Here a replica of the basic XOR is added to the original XOR circuit to allow the A and B inputs to be applied to both the first and second levels of transistors so that the aggregate delays, $\tau_{A}^{\prime}$ and $\tau_{B}^{\prime}$, are now equal. That is, $\tau_{A}^{\prime}$ and $\tau_{B}^{\prime}$ are a result of both the first and second level to output delays. For example, when inputs $\mathrm{A}+$ and B+ are high, then in Figure 4.4 transistors Q3, Q5, Q10 and Q12 are on to pull Vo+ low. If the same bias current is used with the SXOR circuit as was used in the XOR circuit, then the load resistors must be halved to produce the same output $\Delta V$ as the current passing through them is doubled.

Q1, Q2 and Q9 form a basic current mirror circuit to bias the switching transistors of the SXOR circuit. The emitter length of these transistors was increased to $10.3 \mu \mathrm{m}$ (i.e., width/length/emitters $=0.4 \mu \mathrm{m} / 10.3 \mu \mathrm{m} / 4$ ). This extended the constant 
current operation of the mirror transistors Q2 and Q9 from a $V c e=0.5 \mathrm{~V}$ down to $V c e=0.2 \mathrm{~V}$.

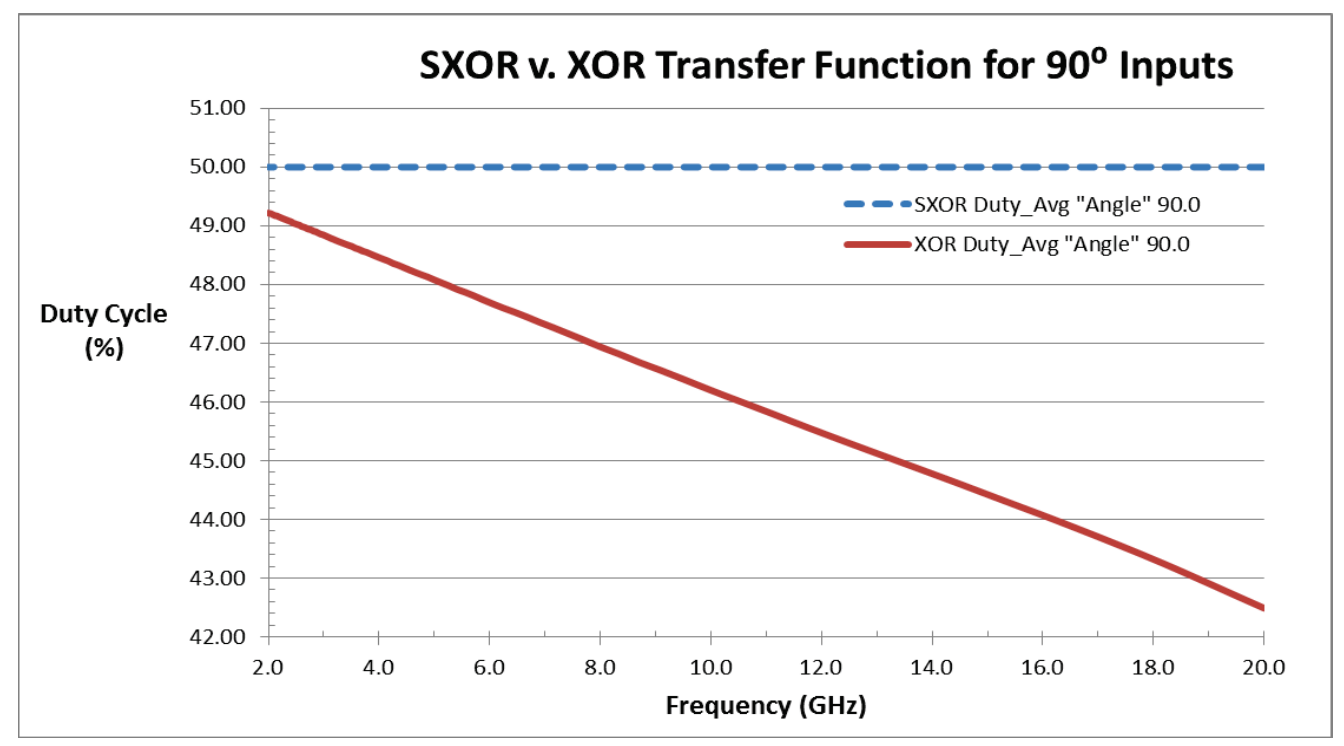

Figure 4.5: SXOR and XOR Output Duty Cycles for $90^{\circ}$ Inputs v. Frequency

Figure 4.5 shows the simulated output duty cycles of the XOR of Figure 4.3 and the SXOR of Figure 4.4 for an input phase difference of $90^{\circ}$ over a frequency range of $2.0 \mathrm{GHz}$ to $20.0 \mathrm{GHz}$. All transistors are minimum size high speed, high current bipolar devices described earlier. The input clock signals are square waves with an amplitude of $0.5 \mathrm{~V}$ and rise/fall times of $1 \mathrm{ps}$. The transient simulation output signals are averaged over the period of $4 n s$ to $6 n s$ for each point plotted. The SXOR plot of Figure 4.5 illustrates a significant improvement in accuracy over the XOR plot. The $0.78 \%$ duty cycle error at $2.0 \mathrm{GHz}$ corresponds to $1.4^{\circ}$ of phase error and the $7.51 \%$ duty cycle error at $20.0 \mathrm{GHz}$ corresponds to $13.6^{\circ}$ of phase error. This increase in error with frequency is nearly linear, which indicates that this error is almost completely due an increase in transit delay $\left(\tau_{F}\right)$ with frequency. The interconnect trace delay (i.e., $\tau_{\text {Trace }}$ ) of equation (4.6) is dependent on microstrip TL geometry and the thickness and relative permittivity of the TL dielectric, and is independent 
of frequency [34]. Therefore, in a realization of the XOR circuit this delay would increase the error of Figure 4.5 by a fixed amount. The SXOR topology will also compensate for this fixed error.

Figure 4.6 is similar to Figure 4.5 except that the input phase difference is $45^{\circ}$, which should result in a duty cycle of $25 \%$. Here the error at $2.0 \mathrm{GHz}$ is $0.78 \%$ as it was for the $90^{\circ}$ case of Figure 4.5. However, as the frequency increases past 12.0 GHz both SXOR and XOR duty cycle outputs deviate from their expected linear paths. As stated previously, if the frequency of the input clock signals is $f$, then the SXOR/XOR transistors must switch at $2 f$. As the phase relationship between the input clocks approaches $45^{\circ}$ and output duty cycle approaches $25 \%$, the switching of the SXOR/XOR transistors must occur in half the time it did for the $90^{\circ}$ input phase relationship. This effectively doubles the switching frequency again to $4 f$. Unfortunately, at an effective frequency of $48.0 \mathrm{GHz}$ the transistors do not completely switch. This results in the non-linear deviation in the curves of Figure 4.6 .

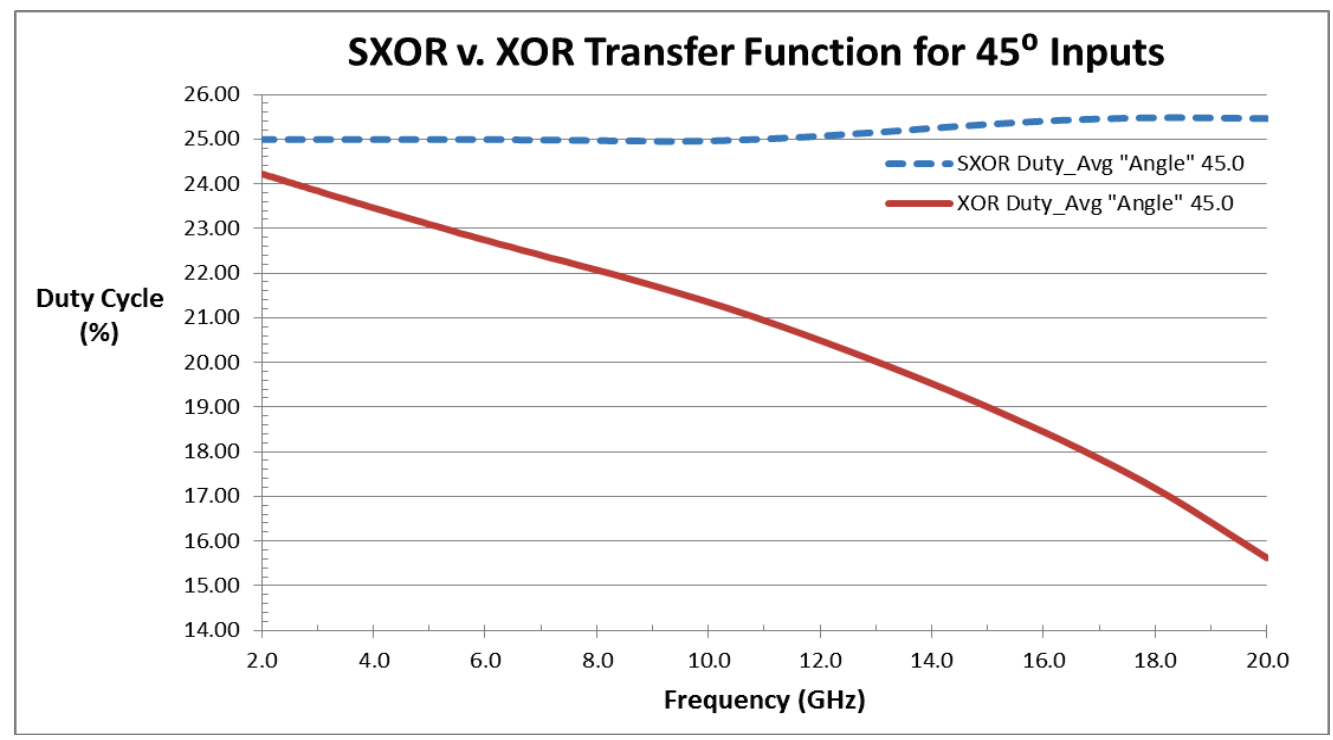

Figure 4.6: SXOR and XOR Output Duty Cycles for $45^{\circ}$ Inputs v. Frequency 


\subsection{Input Biasing and ECL Outputs}

Figure 4.7 shows the SXOR biasing or input level shifting circuit for inputs A and B (Figure 4.4) from the RTWO. The base signals A2+/- and B2+/- are connected to the bases of Q12 to Q15 and Q5 to Q8 of Figure 4.4. Q16 to Q19 provide emitter follower outputs to drive the bases of transistor Q3, Q4, Q10 and Q11 of Figure 4.4. The emitter area of these transistors was increased to width/length/emitters $=0.4 \mu \mathrm{m} / 10.3 \mu \mathrm{m} / 12$ in order to provide enough current to maintain adequate voltage swing at the bases of Q3, Q4, Q10 and Q11. The emitter-follower loads are diode connected minimum size high speed, high current bipolar transistors (i.e., width/length/emitters $=0.4 \mu \mathrm{m} / 1.3 \mu \mathrm{m} / 4$ ) Q20 to Q23 connected to resistors $R b n=10.2 \mathrm{k} \Omega$.

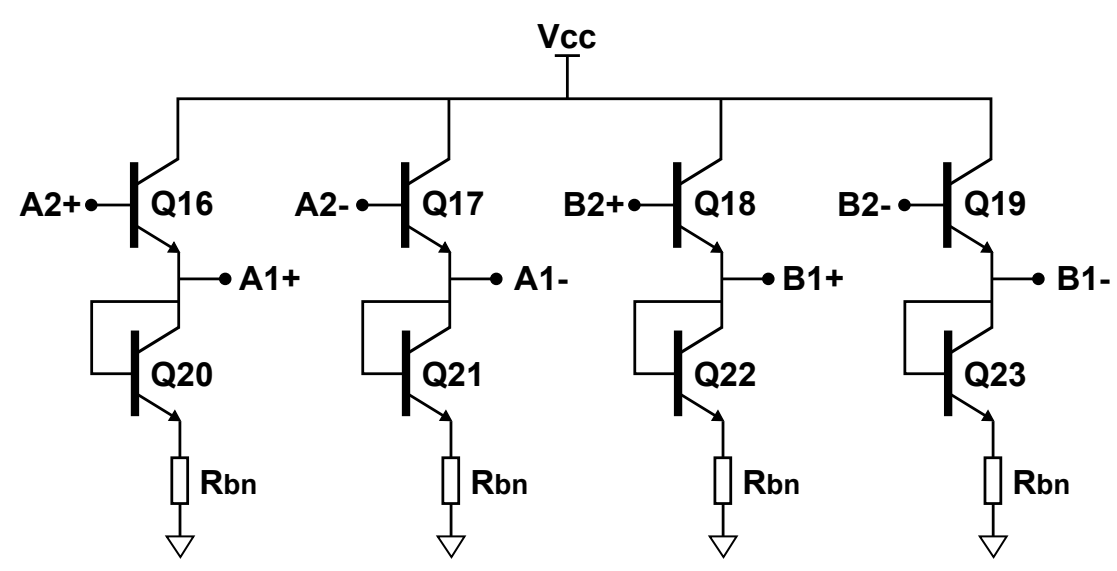

Figure 4.7: SXOR Input Signal Bias Network Schematic

Q24 and Q25 of Figure 4.8 are emitter-follower transistors that isolate the CML outputs of the SXOR from the load. This is important so the switching characteristics of the SXOR are maintained over a range of loads while current is independently provided to any circuit that follows. Additionally, Q24 and Q25 reduce capacitive loading on the CML output node to maintain the speed of the circuit [35]. These transistors change the SXOR from CML at outputs Vo+/- to Emitter-Coupled Logic 
(ECL) at outputs Voe+/-.

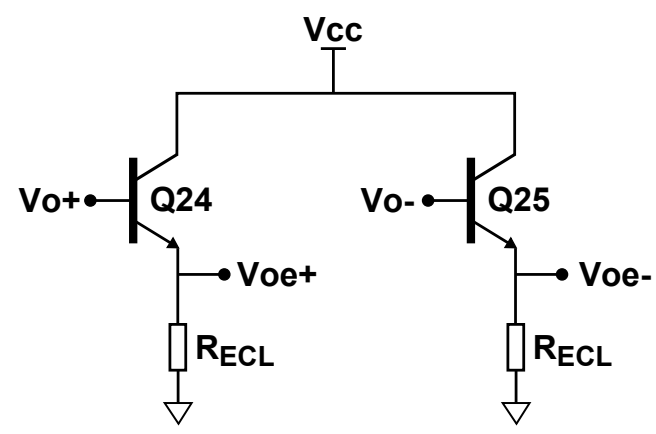

Figure 4.8: SXOR ECL Output Transistor Schematic

\subsection{SXOR Device Layout}

Figure 4.9 illustrates the layout of the SXOR phase detector described in the previous section. This version 2 of the SXOR layout is $162 \mu m \times 130 \mu m$ at its largest points, which is less than half the size of the version 1 device. The CML load resistors, $R_{L} / 2$ of Figure 4.4, are NXP kit type resPD poly $W / L / M=10 \mu m \times 7 \mu m \times 4$ to make $12 \Omega$. The current limit for unsilicided poly is $1 \mu \mathrm{m} / \mathrm{mA}$ for 10 year reliability [36,37]. Therefore, the total resistor width $=4 \times W=4 \times 10 \mu m=40 \mu \mathrm{m}$ gives a $40 \mathrm{~mA}$ limit, which is more than adequate for the $36 \mathrm{~mA}$ flowing through these devices. The common centroid layout (provides some immunity to process mismatch) of these resistor elements is labelled ABBA/BAAB in Figure 4.9 [38]. Additionally, there are four dummy resistors labelled $R_{\text {Dum }}$ across the top of the layout that help with fabrication consistency of these and the emitter-follower output load resistors $R_{E C L}$. As a precaution, all $R_{\text {Dum }}$ resistors are connected to ground on both sides. Resistors $R_{E C L}$ are NXP kit type resPB [36] of size $W / L / M=5 \mu m \times 9.4 \mu m \times 2$ to make $300 \Omega$. The total resistor width $=2 \times 5 \mu m=10 \mu m$ gives a $10 ~ m A$ reliability limit, which is more than adequate for the $6.3 \mathrm{~mA}$ of rms current flowing through these 
devices.

While the SXOR topology equalizes the signal paths running vertically across the device, it does not address differences in the horizontal signal path lengths. This is achieved by making the signal paths lengths from input A2+ to Q12/Q15 = input A2- to $\mathrm{Q} 13 / \mathrm{Q} 14=$ input B2- to $\mathrm{Q} 6 / \mathrm{Q} 7=$ input B2+ $=\mathrm{Q} 5 / \mathrm{Q} 8$; and input $\mathrm{A} 2+$ to $\mathrm{Q} 3=$ input A2- to Q4 = input B2- to Q11 = input B2+ to Q10. Some examples are illustrated in Figure 4.9. Care was taken to include via height when calculating the total length of these paths.

Dummy Resistors were not considered necessary for the input biasing network resistors $R_{b n}$ as their specific values are not critical. They were realized using NXP kit type resPZ [36] of size $W / L / M=1 \mu m \times 5.1 \mu m \times 1$ to make $10.2 k \Omega$. The total resistor width of $1.0 \mu \mathrm{m}$ gives a $1.0 \mathrm{~mA}$ reliability limit, which is more than adequate for the $100 \mu A$ of rms current flowing through these devices.

Most signal paths within the SXOR layout were implemented using metal layers M1, M2 or M3 (nominally $737 \mathrm{~nm}$ thick) as they connect more directly to the transistors than layers M5 (nominally $2055 \mathrm{~nm}$ thick) and M6 (nominally $3055 \mathrm{~nm}$ thick). These traces were made wider than minimum to improve their $I^{2} R$ losses. The trace capacitance to substrate ground was not considered an issue because significant charging currents were being switched. All traces cross each other at $90^{\circ}$ to minimize inductive coupling. M5 was used to create a ground ring, which is tied down to substrate ground around the SXOR device. Ibias and Vcc, as well as Voe+/- were implemented using M6 [37]. 


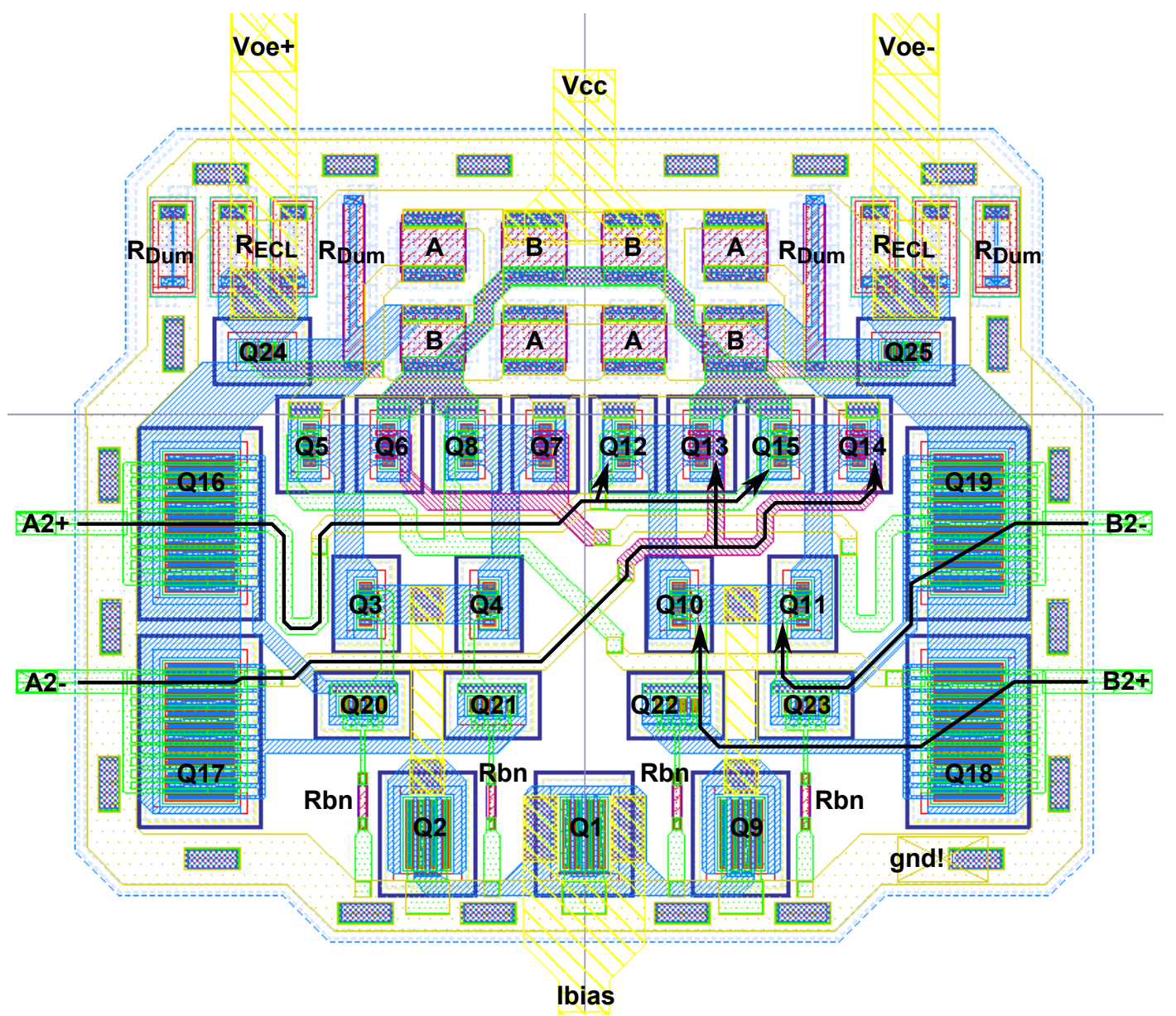

Figure 4.9: Layout of SXOR with ECL Outputs

\subsection{Phase Detector Rev1 versus Rev2}

Two revisions of the differential phase detector test device, Figure 6.3, were fabricated.

Both these die measure $1.0 \mathrm{~mm} \times 1.0 \mathrm{~mm}$. The inset box shown in Figure 4.10 (revision one, Phdetect_1) marks the boundary of the phase detector portion of the circuit layout, which is $296.4 \mu m \times 154.075 \mu m=45,667.8 \mu m^{2}$. The inset box shown in Figure 4.11 (revision two, Phdetect_2) marks the boundary of the phase detector portion of the circuit layout, which is $161.175 \mu m \times 128.675 \mu m=20,739.2 \mu m^{2}$. 


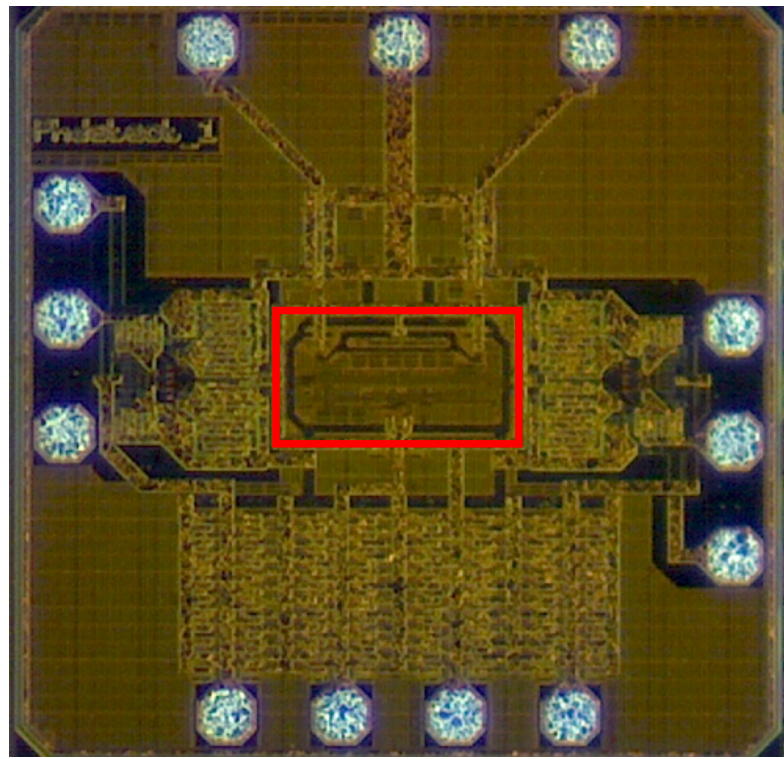

Figure 4.10: SXOR Phase Detector Version One Die (Phdetect_1)

This is a layout area reduction of $56 \%$, which was achieved by repositioning transistors to allow shorter and more symmetrical trace routing.

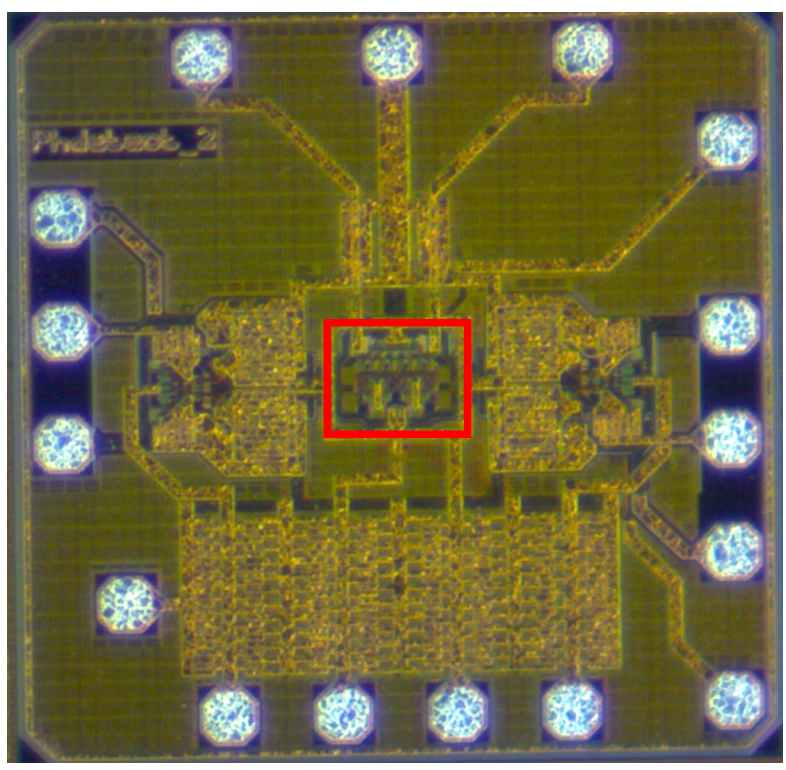

Figure 4.11: SXOR Phase Detector Version Two Die (Phdetect_2) 


\section{Chapter 5}

\section{RTWO Phase Tuning Verification}

\subsection{Extracted RTWO Phase Detector Verification}

Figure 5.1 is a functional block diagram of the simulation test bench used to verify the performance of the extracted ECL SXOR phase detector. This verification has two objectives: first, to verify the monotonicity of the output voltage $V \phi$ for an Input Phase $\Delta$ ramp; and second, to determine the phase detector resolution.

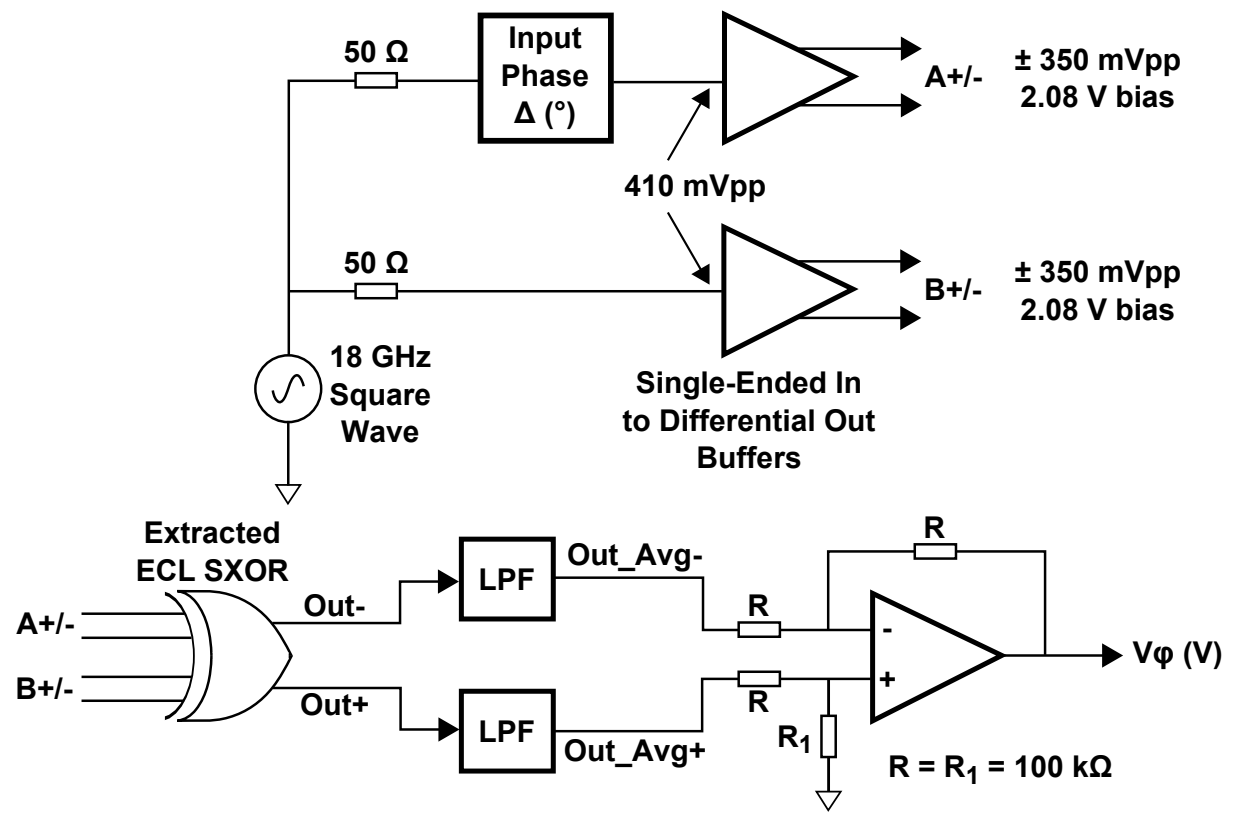

Figure 5.1: Simulation Test Bench for Phase Detector 
The inputs of the Single-Ended In to Differential Out Buffers of Figure 5.1 come from an $18 \mathrm{GHz}$ square wave source that produces $410 m V_{p p}$ at the buffer inputs through $50 \Omega$ source resistances. One input is delayed by the variable Input Phase $\Delta$. The Single-Ended In to Differential Out Buffer circuit implementation is illustrated by Figure 6.1; the layout of the ECL SXOR circuit is illustrated by Figure 4.9; and the LPF circuit implementation is illustrated by Figure 6.2. These three circuit elements are the main components of the device shown in Figure 6.3. The output Op Amp is shown here to represent the equation Out_Avg $+-O u t_{-} A v g-=V \phi$. The Monte Carlo simulations that follow were carried out on extracted versions of these circuits at the corners defined in Table 5.1 over process and mismatch.

Table 5.1: Monte Carlo Simulation Corner Definitions

\begin{tabular}{|c|c|c|}
\hline Corner & Temperature & Voltage (Nominal 2.5 V) \\
\hline \hline Fast & $0^{\circ}$ & $+5 \%(2.625 \mathrm{~V})$ \\
\hline Slow & $100^{\circ}$ & $-5 \%(2.375 \mathrm{~V})$ \\
\hline
\end{tabular}

The plots of Figure 5.2 were generated in the following manner. 200 nominal Monte Carlo simulations were run, over process and mismatch, with Input Phase $\Delta=$ $45^{\circ}$, Temperature $=0^{\circ}$ and $V_{C C}=2.625 V$ (i.e., $+5 \%$ ), then runs were selected that include $98.5 \%$ (excluding outliers) of the results between them i.e., Run 10 and Run 136. Parametric sweeps were then carried out using Runs 10 and 136 form $35^{\circ}$ to $55^{\circ}$ in $1.0^{\circ}$ steps. The results are illustrated by Figure 5.2. 


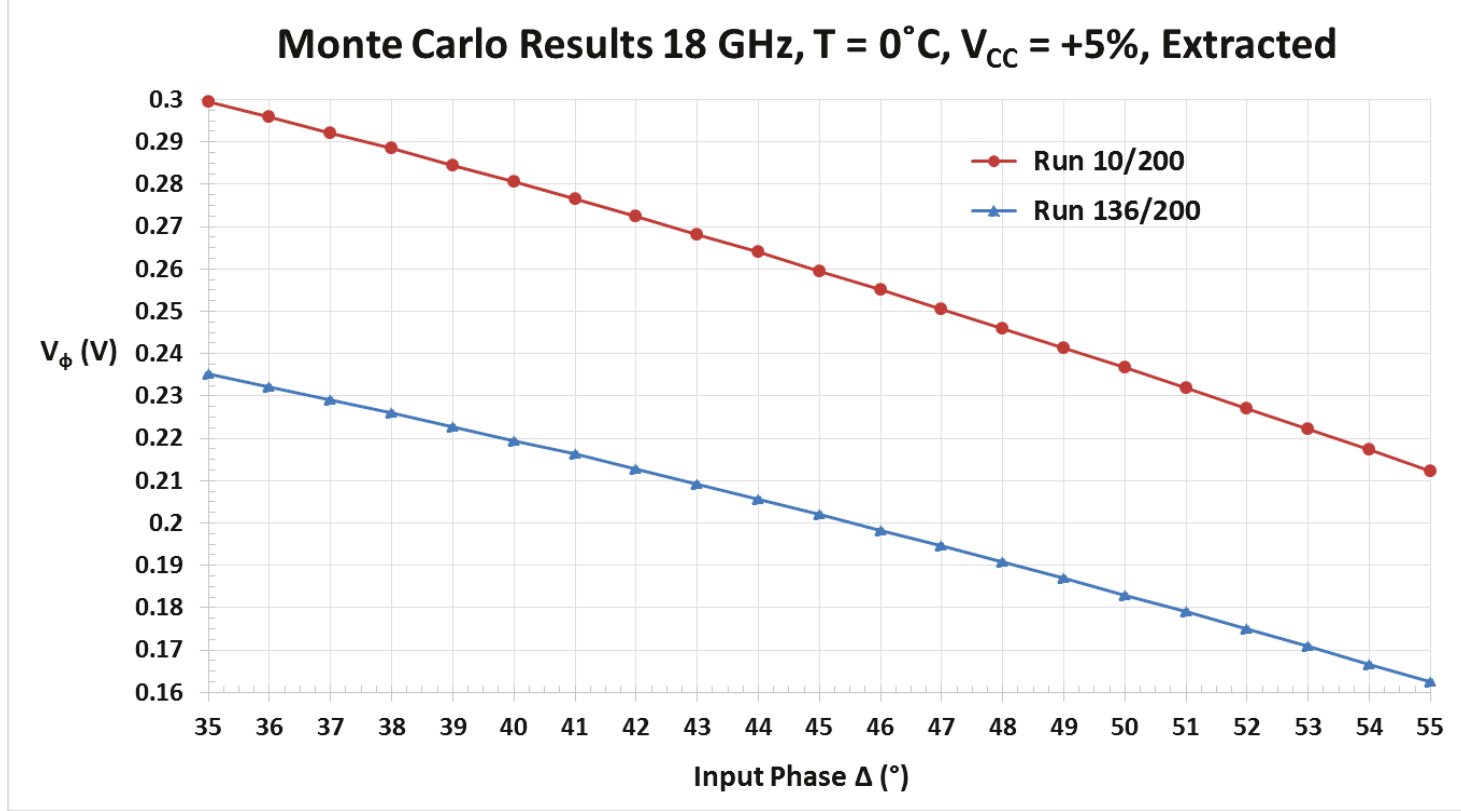

Figure 5.2: Monte Carlo Results for $45^{\circ} \pm 10^{\circ}$, Fast, Extracted

The plots of Figure 5.2 show that the the Output Voltages are clearly monotonic for input increments of $1.0^{\circ}$. They also exhibit a gentle curve or non-linearity caused by incomplete transistor switching around Input Phase $\Delta=45^{\circ} \pm 10^{\circ}$. This was discussed in Section 4.3.

Table 5.2 shows the data that was plotted in Figure 5.2, as well as $\Delta V \phi$, which is the difference between consecutive $V \phi$ voltages over a single run or the $1.0^{\circ}$ output voltage step size. The Range shows how much the output voltage step size varies, due to the non-linearity discussed earlier, over the Input Phase $\Delta$ range. The Average step size and associated Standard Deviation (SD) are also calculated for each run. Here the SD values indicate that the averages are a good representation of the data. The + and - Tolerances show how much the Average output voltage step sized varies over the range of $35^{\circ}$ to $55^{\circ}$. That is, for Run 136 the average step size is $3.6 \mathrm{mV}+15.70 \% /-20.11 \%$ and for Run 10 the average step size is $4.4 m V+14.81 \% /-19.63 \%$. 
These results show that using these SXOR phase detectors to make absolute phase measurements (i.e., measurements to an absolute standard) is not possible without a calibration scheme. For example, Figure 5.2 shows that the $V \phi$ voltage for an InputPhase $\Delta$ of $35^{\circ}$ with Run 136 is approximately the same as the $V \phi$ voltage for an InputPhase $\Delta$ of $50^{\circ}$ with Run 10 . However, if one makes the assumption that the temperature, voltage, process and mismatch variation of a single Monte Carlo Run will be consistent over the physical area of an RTWO on a single die, it is possible to make accurate relative phase measurements using these SXOR phase detectors. That is, a number of SXOR phase detectors can be used to measure the phases difference across TL sections, that should have nominally equal electrical length, around an RTWO. The tolerances of the two extreme runs shown in Table 5.2 are very similar and are less than $\pm 50 \%$; therefore, relative phase tuning to within $1.0^{\circ}$ should be possible with this implementation. 
Table 5.2: Summary of Monte Carlo Results for $45^{\circ} \pm 10^{\circ}$, Fast, Extracted

\begin{tabular}{|c|c|c|c|c|}
\hline $\begin{array}{c}\text { Input } \\
\text { Phase } \Delta\left(^{\circ}\right)\end{array}$ & $\begin{array}{c}\mathrm{V} \phi(\mathrm{V}) \\
\text { Run } 136\end{array}$ & $\Delta \mathbf{V} \phi(\mathbf{V})$ & $\begin{array}{l}\mathrm{V} \phi(\mathrm{V}) \\
\text { Run } 10\end{array}$ & $\Delta \mathbf{V} \phi(\mathbf{V})$ \\
\hline 35 & 0.2351 & - & 0.2994 & - \\
\hline 36 & 0.2322 & 0.0029 & 0.2959 & 0.0035 \\
\hline 37 & 0.2291 & 0.0031 & 0.2922 & 0.0037 \\
\hline 38 & 0.2260 & 0.0031 & 0.2884 & 0.0038 \\
\hline 39 & 0.2228 & 0.0032 & 0.2845 & 0.0039 \\
\hline 40 & 0.2195 & 0.0033 & 0.2806 & 0.0039 \\
\hline 41 & 0.2162 & 0.0033 & 0.2765 & 0.0041 \\
\hline 42 & 0.2127 & 0.0035 & 0.2724 & 0.0041 \\
\hline 43 & 0.2092 & 0.0035 & 0.2682 & 0.0042 \\
\hline 44 & 0.2057 & 0.0035 & 0.2639 & 0.0043 \\
\hline 45 & 0.2020 & 0.0037 & 0.2595 & 0.0044 \\
\hline 46 & 0.1983 & 0.0037 & 0.2550 & 0.0045 \\
\hline 47 & 0.1946 & 0.0037 & 0.2505 & 0.0045 \\
\hline 48 & 0.1908 & 0.0038 & 0.2460 & 0.0045 \\
\hline 49 & 0.1869 & 0.0039 & 0.2413 & 0.0047 \\
\hline 50 & 0.1830 & 0.0039 & 0.2367 & 0.0046 \\
\hline 51 & 0.1790 & 0.0040 & 0.2319 & 0.0048 \\
\hline 52 & 0.1749 & 0.0041 & 0.2271 & 0.0048 \\
\hline 53 & 0.1709 & 0.0040 & 0.2222 & 0.0049 \\
\hline 54 & 0.1667 & 0.0042 & 0.2173 & 0.0049 \\
\hline 55 & 0.1625 & 0.0042 & 0.2123 & 0.0050 \\
\hline \multicolumn{2}{|c|}{ Range (V) } & 0.0013 & - & 0.0015 \\
\hline \multicolumn{2}{|c|}{ Average (V) } & 0.0036 & - & 0.0044 \\
\hline \multicolumn{2}{|c|}{$\mathrm{SD}(\mathrm{V})$} & $380 \times 10^{-6}$ & - & $430 \times 10^{-6}$ \\
\hline \multicolumn{2}{|c|}{+ Tolerance $(\%)$} & 15.70 & - & 14.81 \\
\hline \multicolumn{2}{|c|}{ - Tolerance (\%) } & 20.11 & - & 19.63 \\
\hline
\end{tabular}


The plots shown in Figure 5.3 were generated with the same process used to generate the results of Figure 5.2 except Temperature $=100^{\circ}$ and $V_{C C}=2.375 \mathrm{~V}$ (i.e., $-5 \%$ ). Runs were selected that include $97.5 \%$ (excluding outliers) of the results between them i.e., Run 52 and Run 136.

The plots of Figure 5.3 are monotonic for input increments of $1.0^{\circ}$ and show less curve or better linearity than the plots of Figure 5.2. This is also indicated by the smaller Range values of Table 5.3. The maximum run, Run 52, of Figure 5.3 is similar to the minimum run, Run 136, of Figure 5.2, which further indicates the difficulty in using this SXOR phase detector for absolute measurements without a calibration scheme.

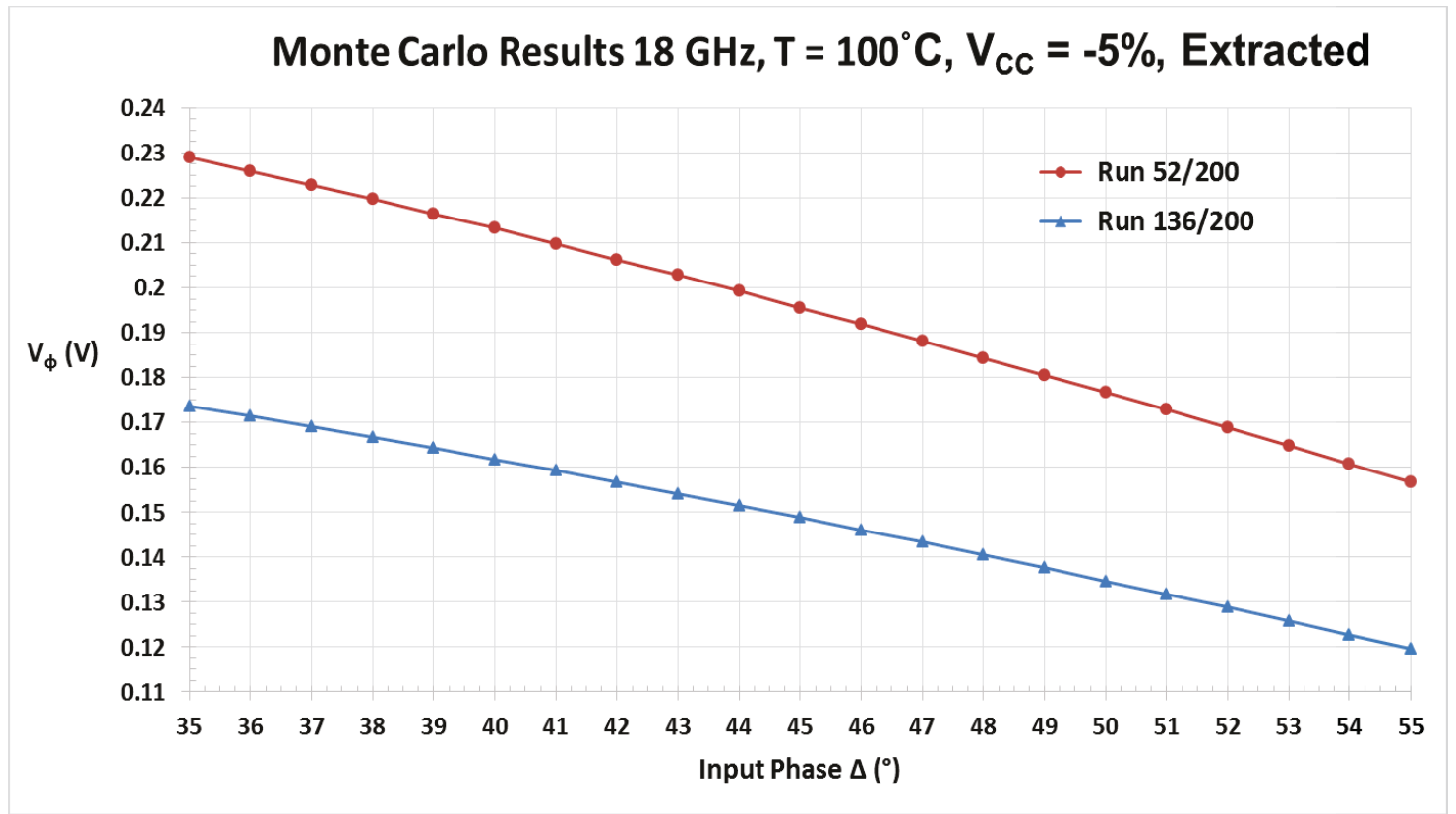

Figure 5.3: Monte Carlo Results for $45^{\circ} \pm 10^{\circ}$, Slow, Extracted

The SD values of Table 5.3 indicate that the averages are a good representation of the data. The average step size for Run 136 is $2.7 \mathrm{mV}+15.03 \% /-18.37 \%$ and for Run 10 the average step size is $3.6 m V+13.26 \% /-14.36 \%$. As was stated previously, the tolerances of the two extreme runs shown in Table 5.3 are very similar and are 
less than $\pm 50 \%$; therefore, relative phase tuning to within $1.0^{\circ}$ should be possible with this implementation.

Table 5.3: Summary of Monte Carlo Results for $45^{\circ} \pm 10^{\circ}$, Slow, Extracted

\begin{tabular}{|r|c|c|}
\hline \multicolumn{1}{|c|}{ Parameter } & Run 136 $\Delta \mathbf{V} \phi(\mathbf{V})$ & Run 10 $\Delta \mathbf{V} \phi(\mathbf{V})$ \\
\hline \hline Range (V) & 0.0009 & 0.0010 \\
\hline Average (V) & 0.0027 & 0.0036 \\
\hline SD (V) & $256 \times 10^{-6}$ & $317 \times 10^{-6}$ \\
\hline + Tolerance (\%) & 15.03 & 13.26 \\
\hline - Tolerance (\%) & 18.37 & 14.36 \\
\hline
\end{tabular}

The procedure for generating Figures 5.4 and 5.5 and Table 5.4 is the same as the procedure discussed earlier in this section except that the Input Phase $\Delta=90^{\circ} \pm 10^{\circ}$.

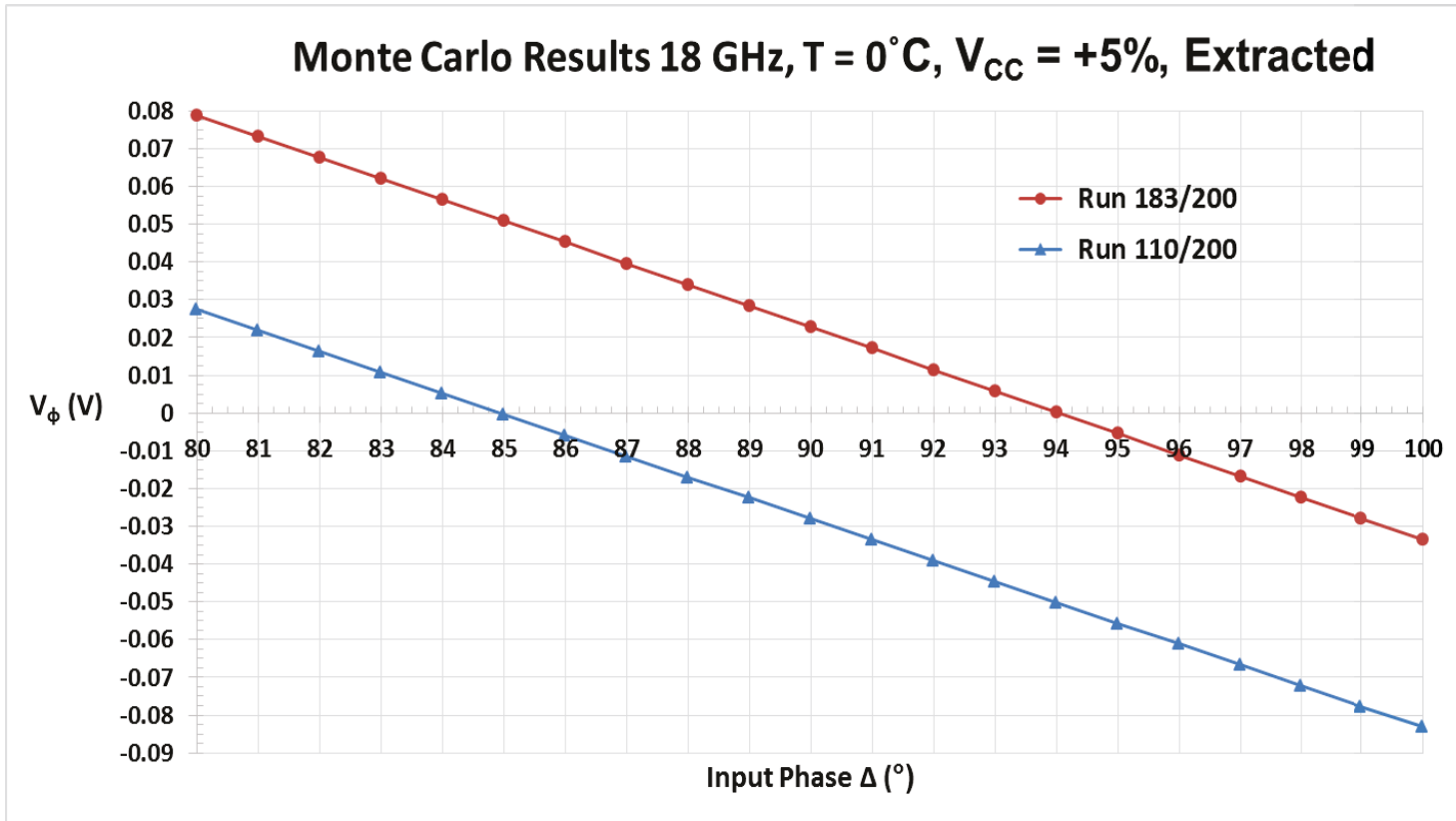

Figure 5.4: Monte Carlo Results for $90^{\circ} \pm 10^{\circ}$, Fast, Extracted

Runs 183 and 110 of Figure 5.4, and runs 186 and 173 of Figure 5.5 encompass $100 \%$ of the Monte Carlo results, i.e., no outliers. There is very little discernible curve 
in these plots, which indicates that at a nominal $90^{\circ}$ phase difference the transistors are switching completely on and completely off at $18 \mathrm{GHz}$. This improvement in linearity over the $45^{\circ}$ cases is quantified by the relatively small Range of $0.0002 \mathrm{~V}$ listed in Table 5.4.

Fast Run 183 crosses $V \phi=0 V$ at $94^{\circ}$ and Fast Run 110 crosses $V \phi=0 V$ at $85^{\circ}$, while Slow Run 186 crosses $V \phi=0 V$ at $95^{\circ}$ and Fast Run 110 crosses $V \phi=0 V$ at $84^{\circ}$. This indicates that there is significant overlap in the Fast and Slow Monte Carlo results when compared to the $45^{\circ}$ simulations.

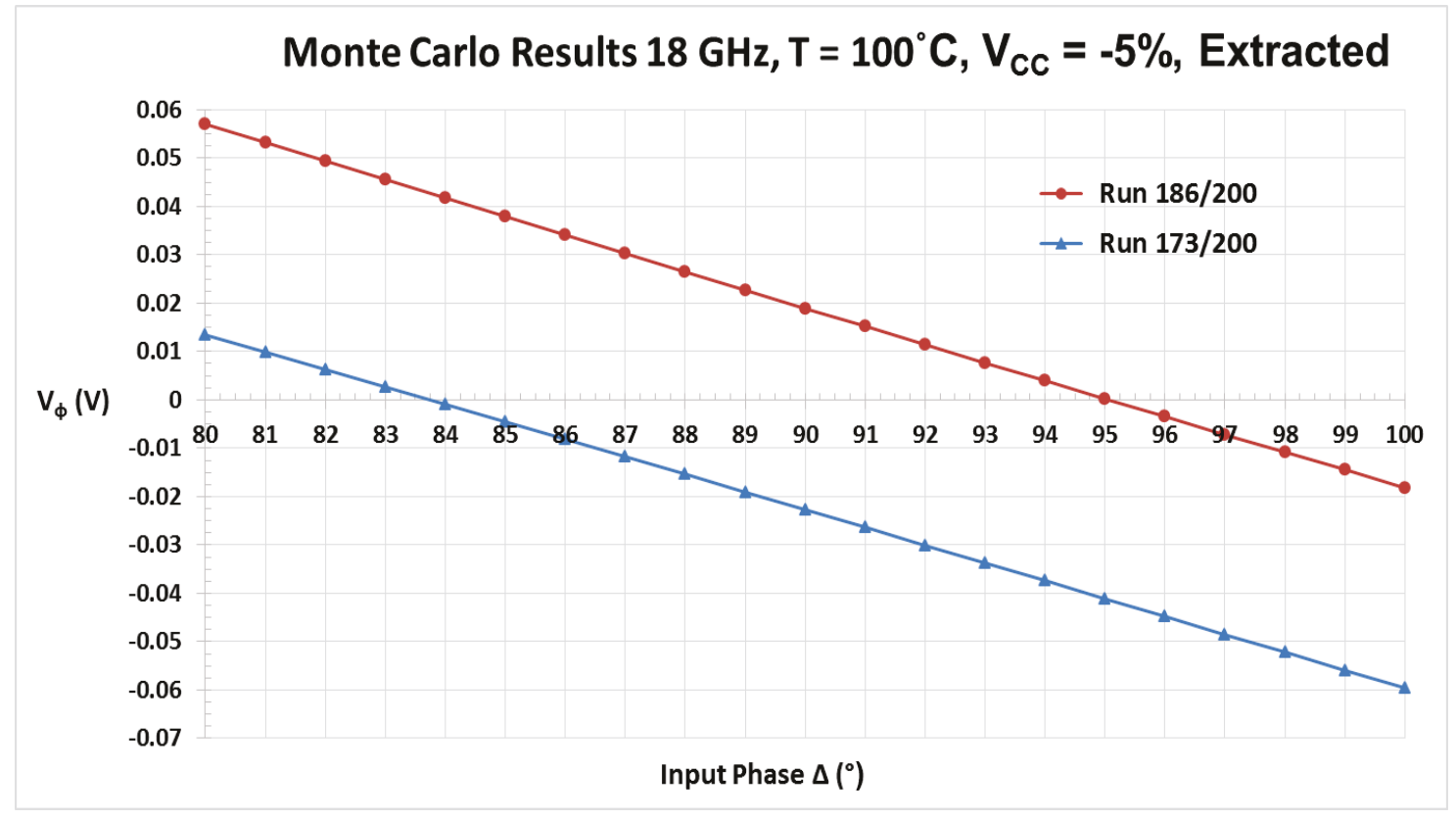

Figure 5.5: Monte Carlo Results for $90^{\circ} \pm 10^{\circ}$, Slow, Extracted

The SD values of Table 5.4 indicate that the averages are a good representation of the data. The + and - Tolerances show how much the Average output voltage step sized varies over the range of $80^{\circ}$ to $100^{\circ}$. These values show a significant improvement in accuracy over the $45^{\circ}$ simulations. Unfortunately, there is significant variation in the average output voltage step size between the fast and slow corners. Therefore, as with the $45^{\circ}$ simulations, absolute phase measurement requires a calibration scheme. 
Table 5.4: Summary of Monte Carlo Results for $90^{\circ} \pm 10^{\circ}$, Extracted

\begin{tabular}{|r|c|c|c|c|}
\hline \multirow{2}{*}{ Parameter } & \multicolumn{2}{|c|}{ Fast V $\phi($ V) (Fig. 5.4) } & \multicolumn{2}{c|}{ Slow V $\phi($ V) (Fig. 5.5) } \\
\cline { 2 - 5 } & Run 183 & Run 110 & Run 186 & Run 173 \\
\hline \hline Range (V) & 0.0002 & 0.0002 & 0.0002 & 0.0002 \\
\hline Average (V) & 0.0055 & 0.0056 & 0.0037 & 0.0038 \\
\hline SD (V) & $42 \times 10^{-6}$ & $45 \times 10^{-6}$ & $50 \times 10^{-6}$ & $64 \times 10^{-6}$ \\
\hline + Tolerance (\%) & 1.08 & 0.77 & 2.68 & 2.11 \\
\hline - Tolerance (\%) & 2.00 & 2.43 & 2.66 & 3.47 \\
\hline
\end{tabular}

Additional nominal Monte Carlo simulations show that the extracted phase detectors performed well in the ranges $45^{\circ} \pm 10^{\circ}$ and $90^{\circ} \pm 10^{\circ}$ up to $20 \mathrm{GHz}$. While the performance of these phase detectors with $90^{\circ} \pm 10^{\circ}$ input phase differences is superior to the performance with $45^{\circ} \pm 10^{\circ}$ input phase differences, this improvement was not considered to be significant enough to rule out using these phase detectors to measure $45^{\circ} \pm 10^{\circ}$ phase differences. Also, additional circuit components (i.e., $\lambda / 8$ transmission lines and transistor switches) would be needed to implemented, characterized and calibrated to use $90^{\circ} \pm 10^{\circ}$ input phase differences in this application.

\subsection{RTWO System Simulation Set-up}

Figure 5.6 is a functional block diagram of the test bench used to simulate the implementation of the RTWO phase alignment circuit that was fabricated. The $250 \mathrm{fs}$, $500 f s$ and $750 \mathrm{fs}$ Delay blocks were used to create a phase error for the system to correct. This was necessary as the RTWO $18 \mathrm{GHz}$ block sections needed to be identical for the simulation to be stable. The four SXOR blocks include two extracted differential input buffers and an extracted SXOR circuit. In order to reduce the number of die pads only one of the filtered signals was used as an output. The 
complementary unused outputs were terminated in $50 \Omega$ resistors. Extracted versions of the LPF blocks were used in this simulation.

The 4-Channel, 12-Bit, Parallel A/D block and Phase Alignment Algorithm block are reused from Figure 3.10. Here the A/D reference voltage is $2.5 \mathrm{~V}$ and the resolution is given by equation (5.1). A $1^{\circ}$ single-ended transfer function step is $\approx 2 \mathrm{mV}$; therefore, the calculated resolution is adequate for this simulation.

$$
2.5 /\left(2^{12}-1\right)=610.5 \mu V
$$

The 4-Channel, 9-Bit, Parallel D/A block has been added to allow the digital control outputs of the phase alignment algorithm to adjust the analogue control inputs of the four varactors (i.e., Vtune_Top/Vtune_Left/Vtune_Bottom/Vtune_Right). The $\mathrm{D} / \mathrm{A}$ reference voltage is $2.5 \mathrm{~V}$ and its resolution is given by equation (5.2).

$$
2.5 /\left(2^{9}-1\right)=4.892 m V
$$

Using equation (2.18), as well as the circuit parameter values of Figure 5.7 and [6], it was determined that an $8 f F$ change in varactor capacitance results in $\approx 0.5^{\circ}$ of change in the electrical phase of a single TL section. The varactor differential capacitance versus control voltage plot of Figure 5.8 shows that, to a first order approximation, the voltage resolution calculated in equation (5.2) will never result in a phase step of more than $0.5^{\circ}$.

The four $5 k \Omega$ resistors connected to the Vtune signals allow these signals to be controlled individually for phase tuning or as a single frequency tuning control signal.

The SXOR phase detector inputs at point A of Figure 5.6 are the same polarity as the other phase detector connections and are reversed from Figure 3.10. If we use the Out- signal of this SXOR, as has been done with the other three SXOR phase 
detectors, the phase measurement would be the sum of the measurements made by those phase detectors. This error has been avoided by using Out+ from this SXOR phase detector.

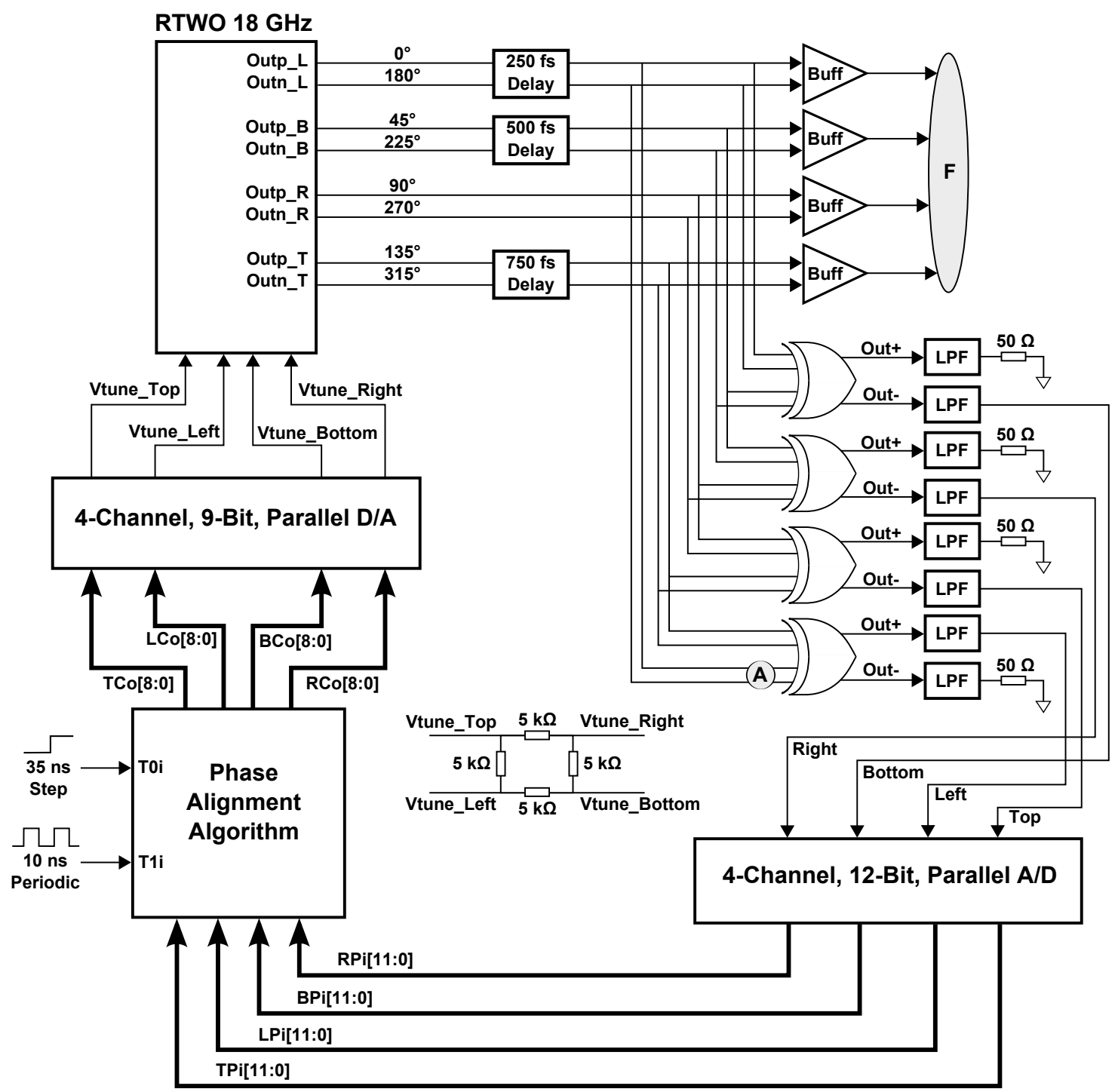

Figure 5.6: AMSD System Model of Correctly Working RTWO Phase Calibration Circuit

The RTWO $18 \mathrm{GHz}$ block transmission lines are realized with lumped element components as shown in Figure 5.7. The component values were determined from $[6,7]$. The oscillator gain and buffer elements, as well as varactors were implemented 
using kit components. These component designs were used in the fabricated RTWO circuit.

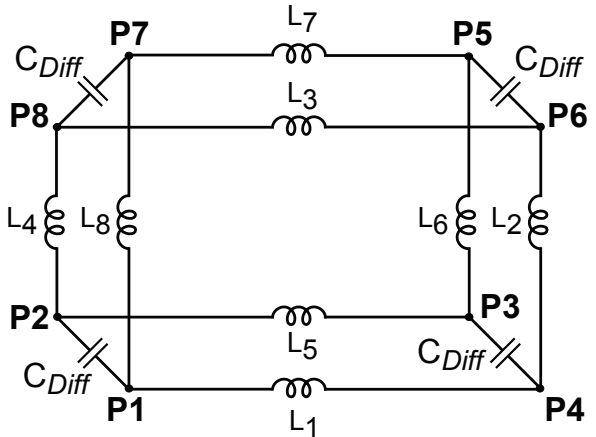

$\mathrm{C}_{\text {Diff }}=18.786 \mathrm{fF}$

$\mathrm{L}_{\text {Diff }} / 2=\mathrm{L}_{\mathrm{n}}=74.678 \mathrm{pH}$

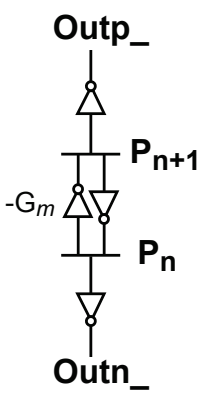

_ indicates:_Right; _Left; _Top; or_Bottom

Series $\mathrm{R}=450 \mathrm{mOhm}$ included in every Inductor $\mathrm{L}$.

Figure 5.7: Lumped Element RTWO LT $[6,7]$ with Kit Varactors [8]

The VcapDNMOSNW component of Figure 5.7 is a differential varactor from the NXP BiCMOS kit [8] with the characteristics illustrated in Figure 5.8. This plot was generated using differential s-parameters.

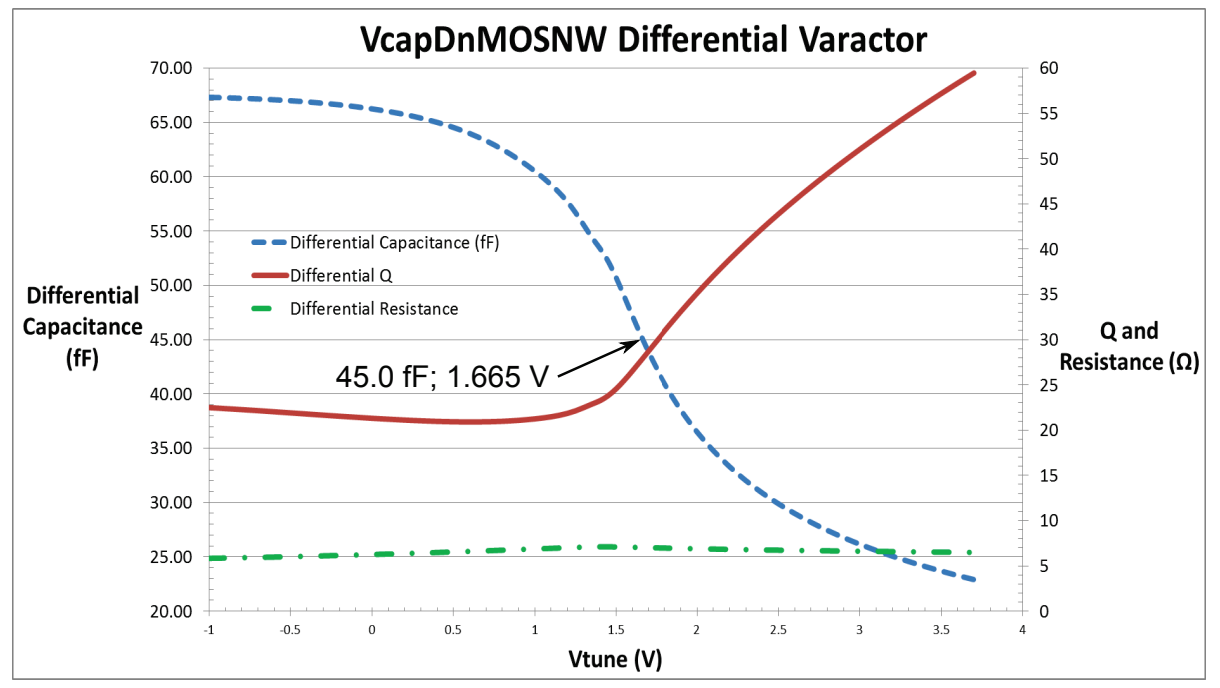

Figure 5.8: Differential C, Q and R for VcapDnMOSNW Kit Varactor

This VcapDnMOSNW component was selected for this application because of its 
large capacitance range and the slope of the capacitance versus control voltage curve is better suited to analogue voltage control (i.e., smaller change in $\mathrm{C}$ for a change in V) than others available in the kit. Also, its symmetry about the half capacitance point marked on the figure will give consistent tuning over its range. The size of this varactor (i.e., $W / L=5 \mu m \times 0.5 \mu m$ with eight stripes) was selected to optimize $\mathrm{Q}$ and capacitance for this application.

\subsection{RTWO System Simulation - Typical Results}

The filtered phase detector results of the extracted AMS Designer system model of Figure 5.6 are illustrated in Figure 5.9. Delay elements of $250 \mathrm{fs}, 500 \mathrm{fs}, 0$ fs and 750 fs were used to create a phase error for the phase alignment algorithm to correct. The system functioned correctly and converged up to the point where the varactor control voltage range was exceeded (i.e., $490 \mathrm{~ns}$ ). 


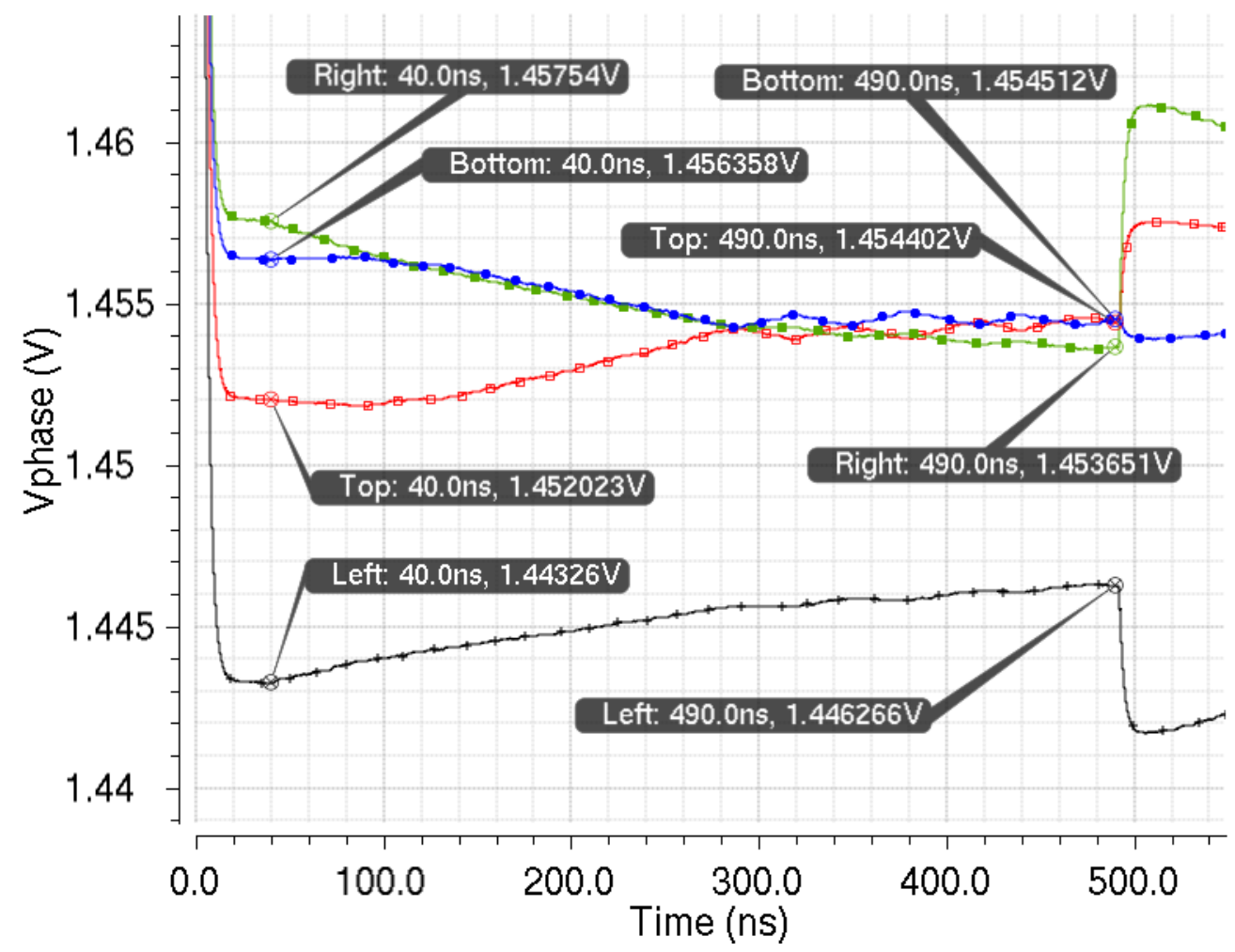

Figure 5.9: Simulated Left, Bottom, Right and Top Signals of Figure 5.6

Figure 5.10 shows the nominal varactor control voltages at each iteration of the phase alignment algorithm. At $490 \mathrm{~ns}$ the algorithm tries to reduce the Left varactor Control signal voltage (Vtune_Left) below zero, which is outside its range of $0 \mathrm{~V}$ to $2.5 \mathrm{~V}$. The digital word that determines the Vtune_Left voltage then flips over to a near maximum voltage. This problem can be resolved by adding additional differential varactor circuits to each TL section to increase the tuning range and by adjusting the RTWO TL lengths to compensate for the resulting operating frequency reduction due to the increase in minimum total varactor capacitance. 


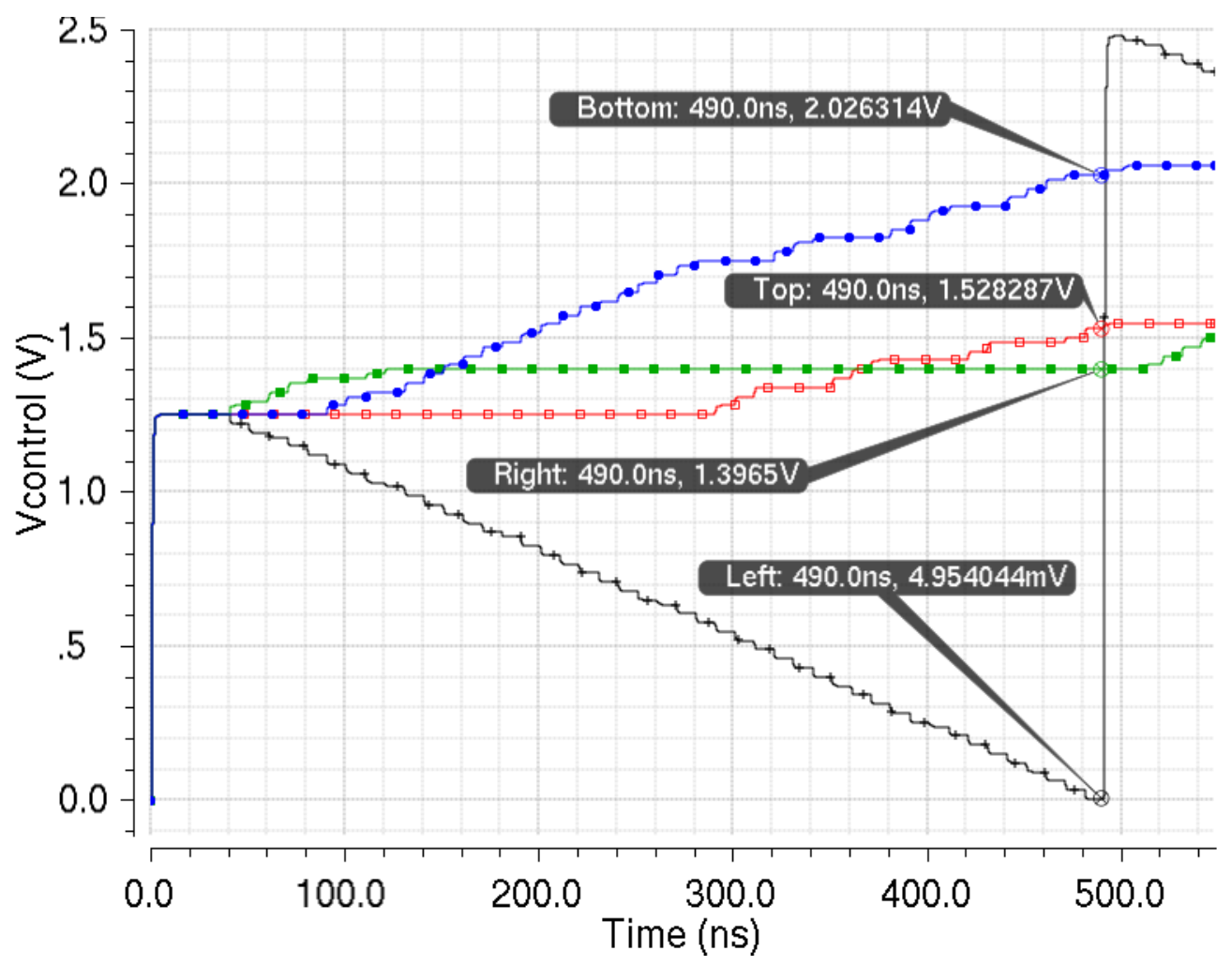

Figure 5.10: Simulated Varactor Control Voltages of Figure 5.6

\subsection{RTWO System Simulation - Updated from Fabrication}

Figure 5.11 is an AMS Designer model of the circuit that was fabricated to produce the die of Figure 6.11. This circuit differs from Figure 5.6 as follows:

1. The system of Figure 5.6 uses a lumped element TL RTWO implementation, Figure 5.7. Here, Figure 5.11, the transmission lines of the TL sections have been implemented in an eight port s-parameter module (i.e., N-Port Network). This module points to the Touchstone ${ }^{\circledR}$ file RTWK_ideal_mlin.s8p, which was generated using ADS Momentum ${ }^{\circledR}$ and the kit substrate to simulate the layout of the RTWO transmission lines. This eight port s-parameter 2.5D simulation 
was run from $0 \mathrm{~Hz}$ to $140 \mathrm{GHz}$ to include at approximately eight harmonics. The s-parameter was used because it improved the stability of this simulation.

2. The portion of the AMS Designer model in the dashed box shown in Figure 5.11, labelled Fabricated Die and excluding the N-Port Network, is a single extracted block. In the model of Figure 5.6 each illustrated circuit block was extracted separately. It should be noted that previous attempts to simulate using this single extracted block failed. This was because the layer one ground plan under the RTWO and oscillator signal distribution transmission lines failed the layout design rules when it was continuous. A significant number of small holes or cut-outs were inserted in the ground plane to satisfy these design rules. Unfortunately, these holes made the ground plane equivalent circuit extraction very complicated, which in turn prevented practical simulation. A second extracted circuit was created with a continuous ground plan and has been used here.

3. The final difference is in the filtered phase detector output signals at points A, $\mathrm{B}$ and $\mathrm{C}$ of Figure 5.11. It was explained earlier that for single-ended operation if the negative output signals were used from the first three differential SXOR circuits, the positive output would have to be used from the fourth SXOR to ensure that all four phases are measured. The filtered Out+ signal of the first SXOR and filtered Out- of the fourth SXOR were what was actually fabricated. 


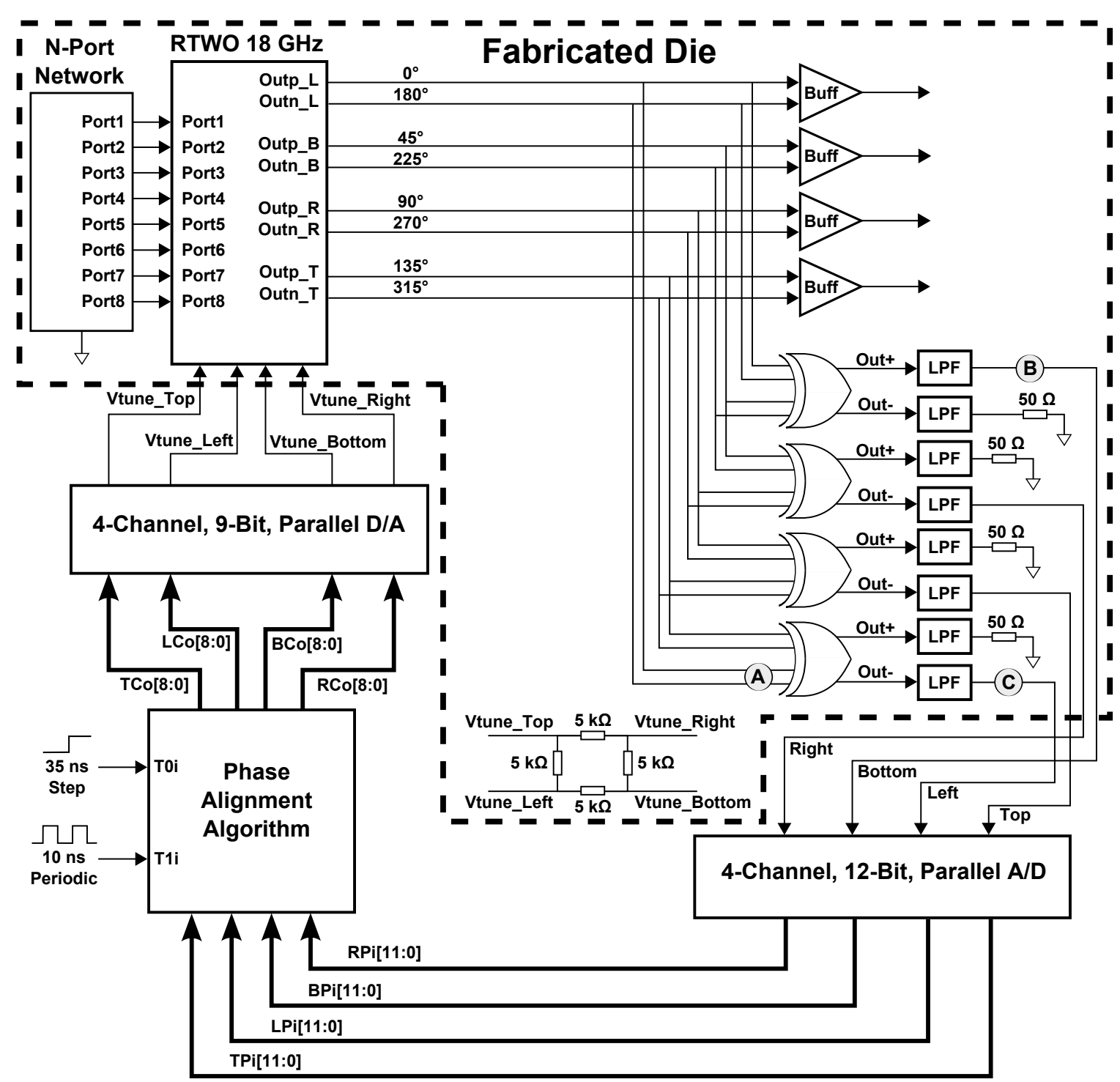

Figure 5.11: AMSD System Model of Implmented RTWO Phase Calibration Circuit

The simulated filtered phase detector results of Figure 5.11 are illustrated in Figure 5.12. Here no artificial delay elements were needed as the circuit layout variation provided finite phase errors. We can see that the system did not converge as it has in previous examples. This is because the phase detector output, point B of Figure 5.11 , is measuring a nominal $135^{\circ}$ instead of $45^{\circ}$, refer to the XOR transfer function of Figure 4.2. Additionally, the corresponding varactor control will need to correct in 
the opposite direction from the other varactor controls.

All four phase detector outputs show various amounts of RF feed-through - the most significant on the signal marked Bottom. This feed-through will be reduced by using the phase detector outputs differentially and could be mitigated further by improvements in the layout to reduce RF coupling.

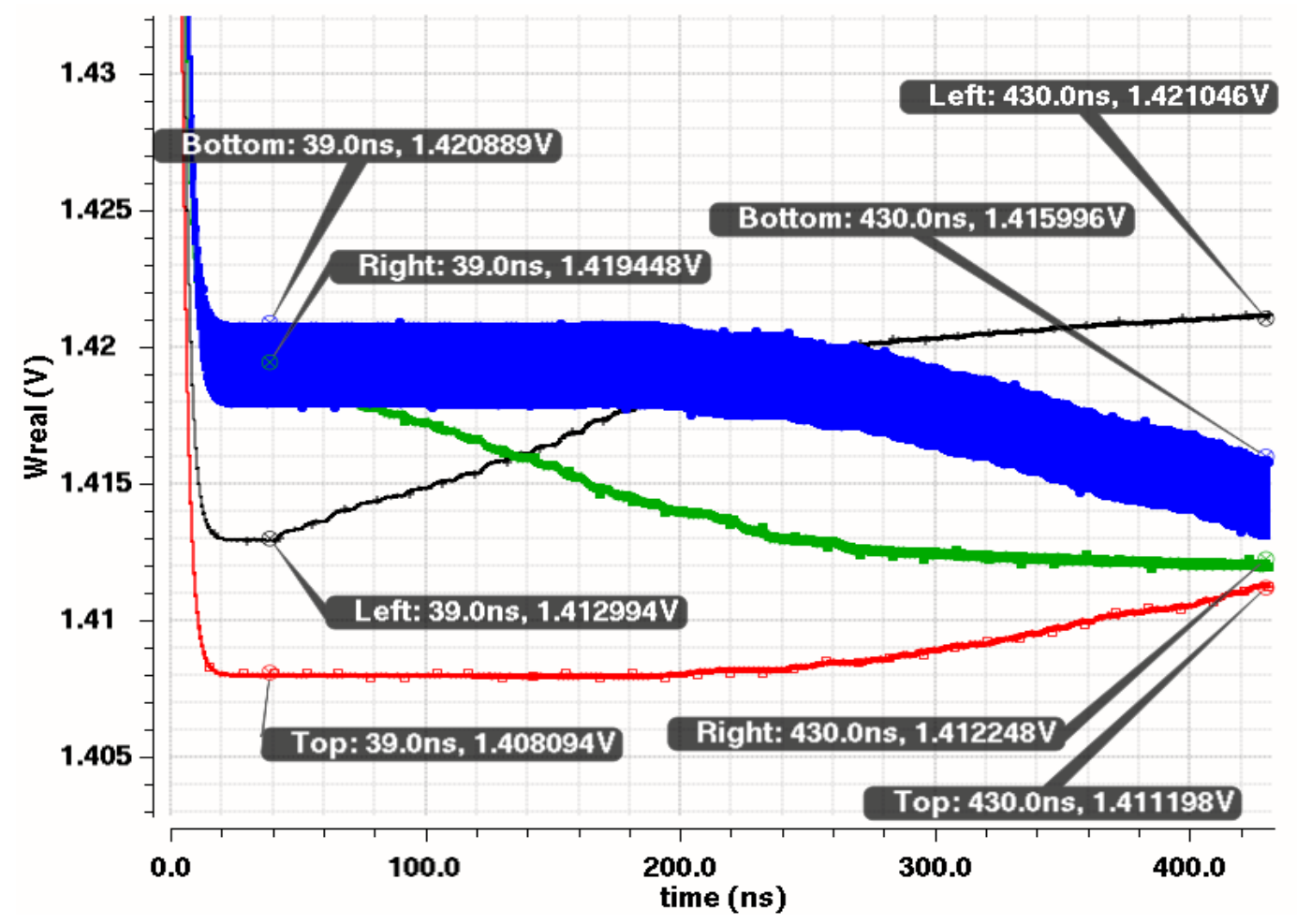

Figure 5.12: Simulated Left, Bottom, Right and Top Signals of Figure 5.11

The varactor control voltage (Vtune_Top, Vtune_Left, Vtune_Bottom and Vtune_Right) simulation results corresponding to Figure 5.12 are shown in Figure 5.13. Here Vtune_Left needs to correct in the opposite direction. 


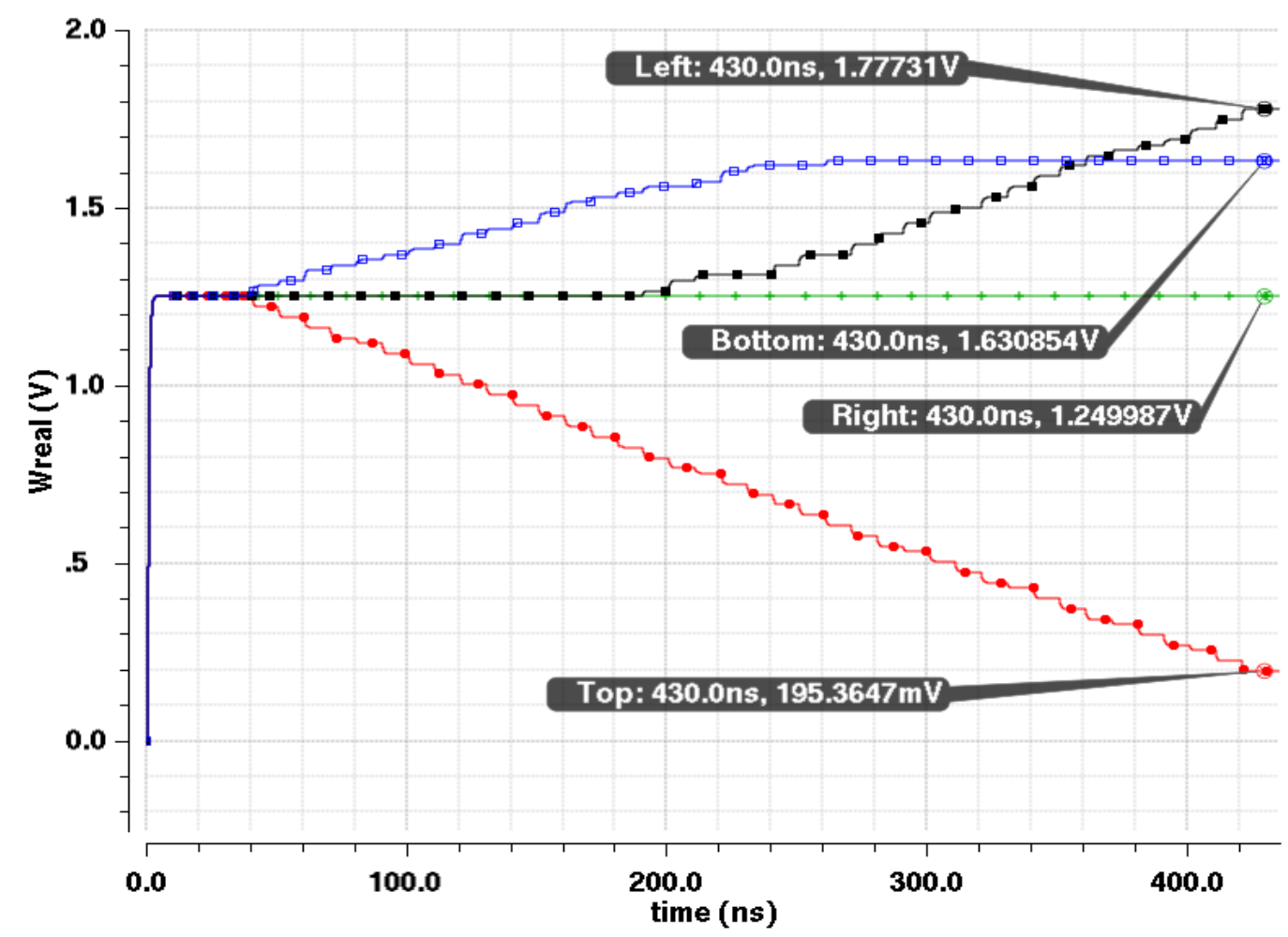

Figure 5.13: Simulated Varactor Control Voltages of Figure 5.11

The simulation results of Figure 5.14 are the phase detector output voltages using the AMS Designer model of Figure 5.6 with the phase detector output signals configured as in Figure 5.11. The objective here was to see if similar results could be obtained using a circuit that had previously given good results. Here we can see that the Left phase detector voltage is diverging as in the previous simulation. In fact, the markers show that all these signal are increasing in voltage with time. These signals are spread further apart as a result of the artificial delay elements. 


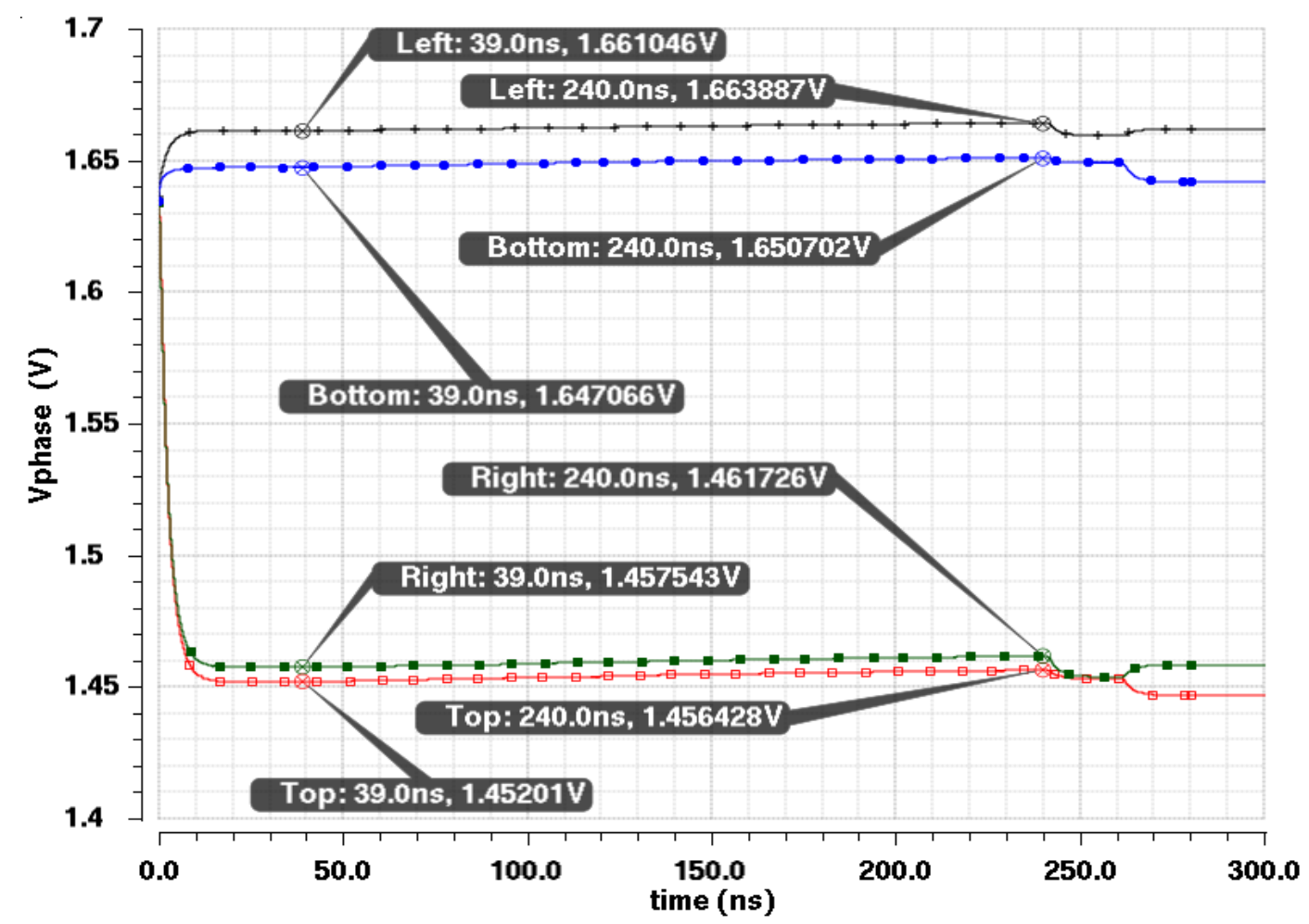

Figure 5.14: Simulated Left, Bottom, Right and Top Signals of Modified Figure 5.6

The varactor control voltage (Vtune_Top, Vtune_Left, Vtune_Bottom and Vtune_Right) simulation results corresponding to Figure 5.14 are shown in Figure 5.15. Here Vtune_Left needs to correct in the opposite direction. Also, we see the result of running out of varactor capacitance at around 250 ns. In a practical application multiple circuit elements would be required to extend the tuning range. 


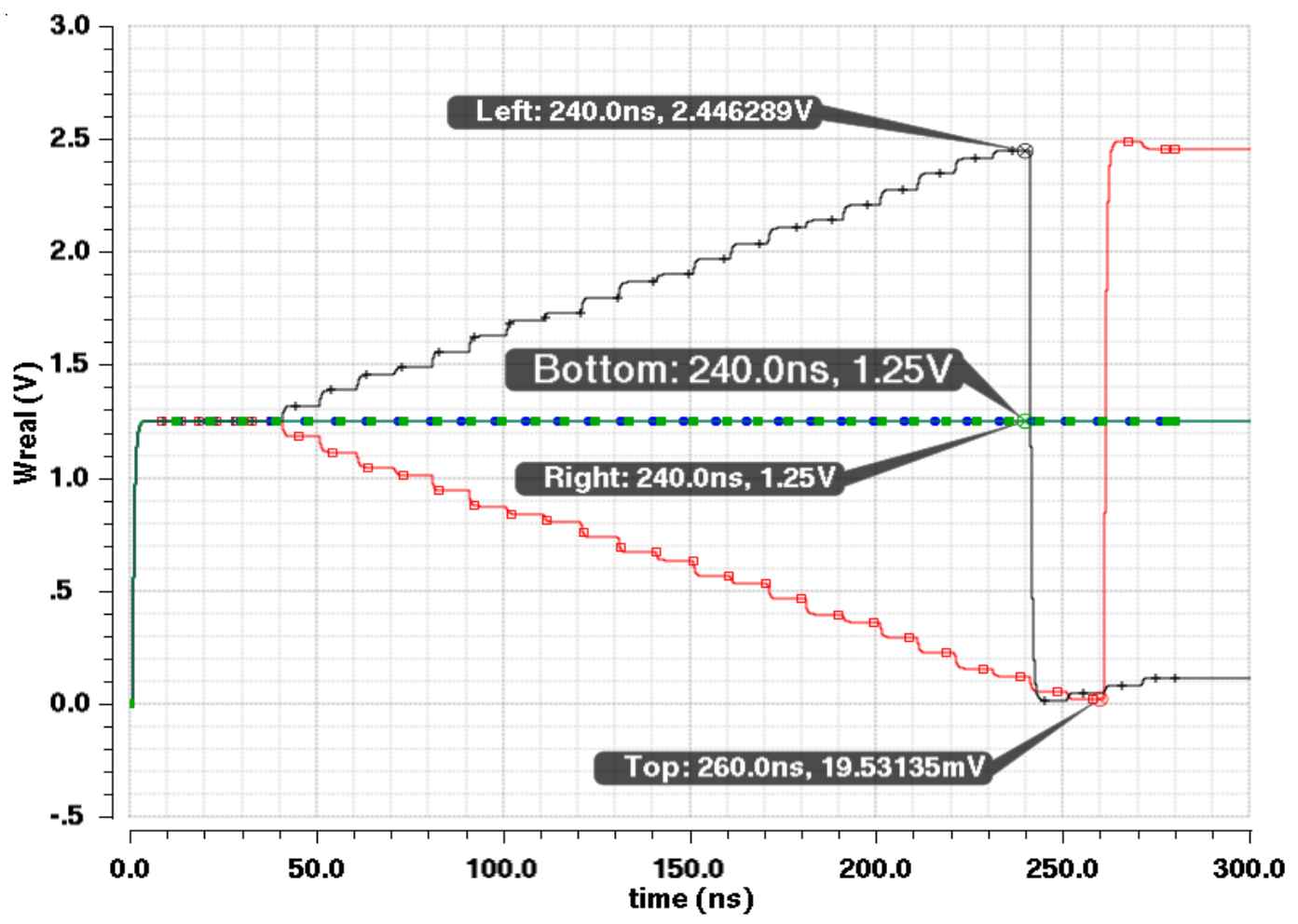

Figure 5.15: Simulated Varactor Control Voltages of Modified Figure 5.6 


\section{Chapter 6}

\section{Experimental Results}

\subsection{SXOR Phase Detector Test Set-up and Proce- dure}

Characterization of the SXOR phase detector is required to determine its accuracy and thus, its suitability for measuring the phase error of an RTWO. In order to provide differential stimulus signals to the SXOR from a single-ended lab signal generator, single-ended to differential buffers were implemented. This buffer circuit is shown in Figure 6.1. It consists of a minimum size transistor differential pair with emitterfollower outputs. The single-ended input to Q3 is dc blocked by a $3 p F$ mimCap so the input can be biased optimally and independently of the external signal generator (i.e., no external bias tee requirement). This configuration is duplicated for Q4 to balance the conversion from single-ended input to differential output. The emitter-follower outputs are also capacitor isolated to allow these differential signals to be biased optimally for the phase detector inputs. The output mimCaps are $10 \mathrm{pF}$ to support wideband testing (i.e., 2.0 GHz to $20.0 \mathrm{GHz}$ ) of the phase detector. Transistors Q1 and Q2 are similar to the current mirror transistors of Figure 4.4. 


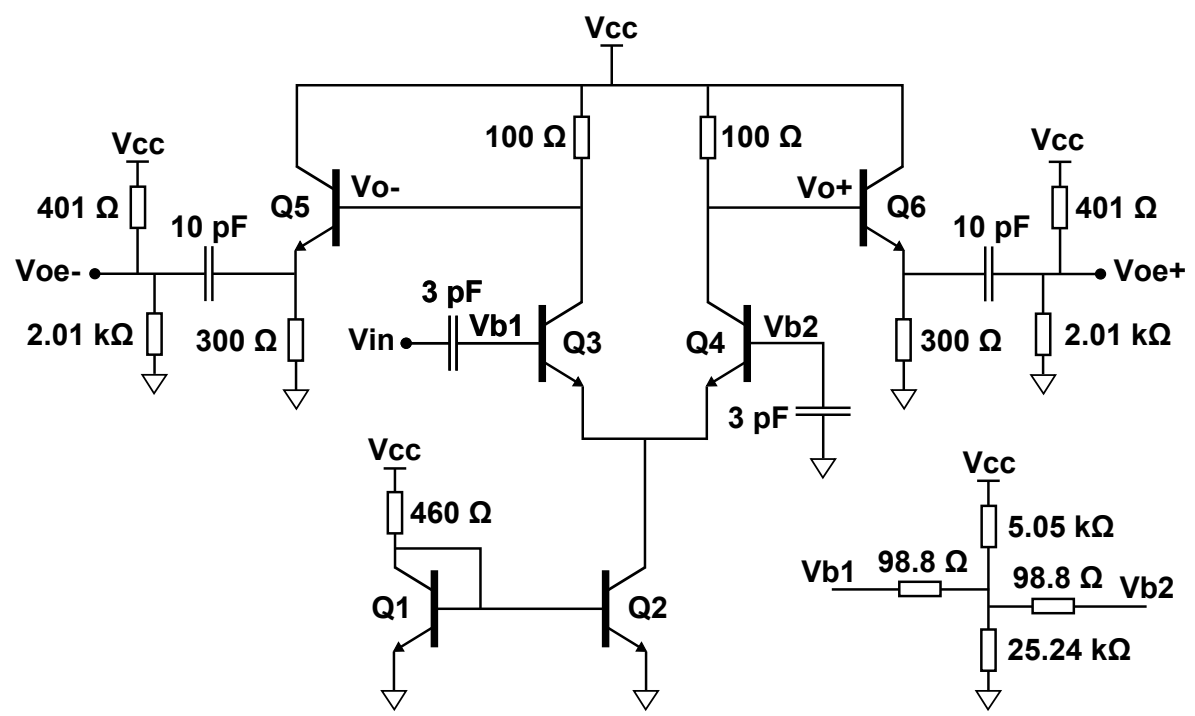

Figure 6.1: Single-Ended Input to Differential Output Buffer

Figure 6.2 is the two-stage LPF used to average the output duty cycle signals of the phase detector. A phase detector output signal will charge the $2 p F$ capacitor through the $1.1 \mathrm{k} \Omega$ resistor to produce an average dc level proportional to the duty cycle. The second $1.1 \mathrm{k} \Omega$ resistor and $200 \mathrm{fF}$ capacitor filter the feed-through signal that is present at twice the input operating frequency. This feed-through is caused by the frequency doubling effect of the XOR phase detector function.

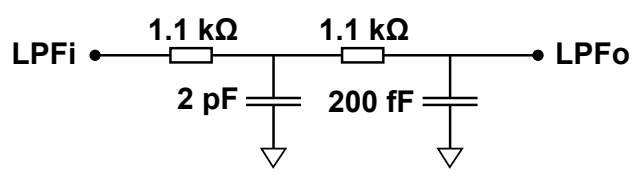

Figure 6.2: Low Pass Filter (LPF)

The circuits of Figures 4.9, 6.1 and 6.2 were combined to produce the test chip illustrated in Figure 6.3. The SXOR phase detector is located in the centre of the Phdetect_2 test device layout. The outputs of the SXOR feed two LPF devices, which in turn feed the pads $V \phi \mathrm{O}+/-$. Around the SXOR phase detector is a $V_{C C}$ ring that supplies the phase detector and two input buffers. This ring is connected to the 
$V_{C C}$ pad and is decoupled by a bank of mimCaps totalling $22 p F$ [39]. Three other $22 p F$ mimCaps are used to decouple Ibias0 for the phase detector, as well as Ibias1 and Ibias2, which are associated with the input single ended to differential output buffers. The high frequency inputs of these buffers are connected to the gnd/sig/gnd (GSG) pads for probing. The Sense_ $V_{C C}$ and Sense_Ibias0 pads are connected to $V_{C C}$ and Ibias0 so the voltage at these points can be more directly measured with a high impedance volt meter. This allows the adjustment of these supplies to compensate for $I^{2} R$ losses in the electrical supply path. The buffers and phase detector are enclosed within a Deep Trench Isolation (DTI) rectangle to isolate them from the supply decoupling [40]. 


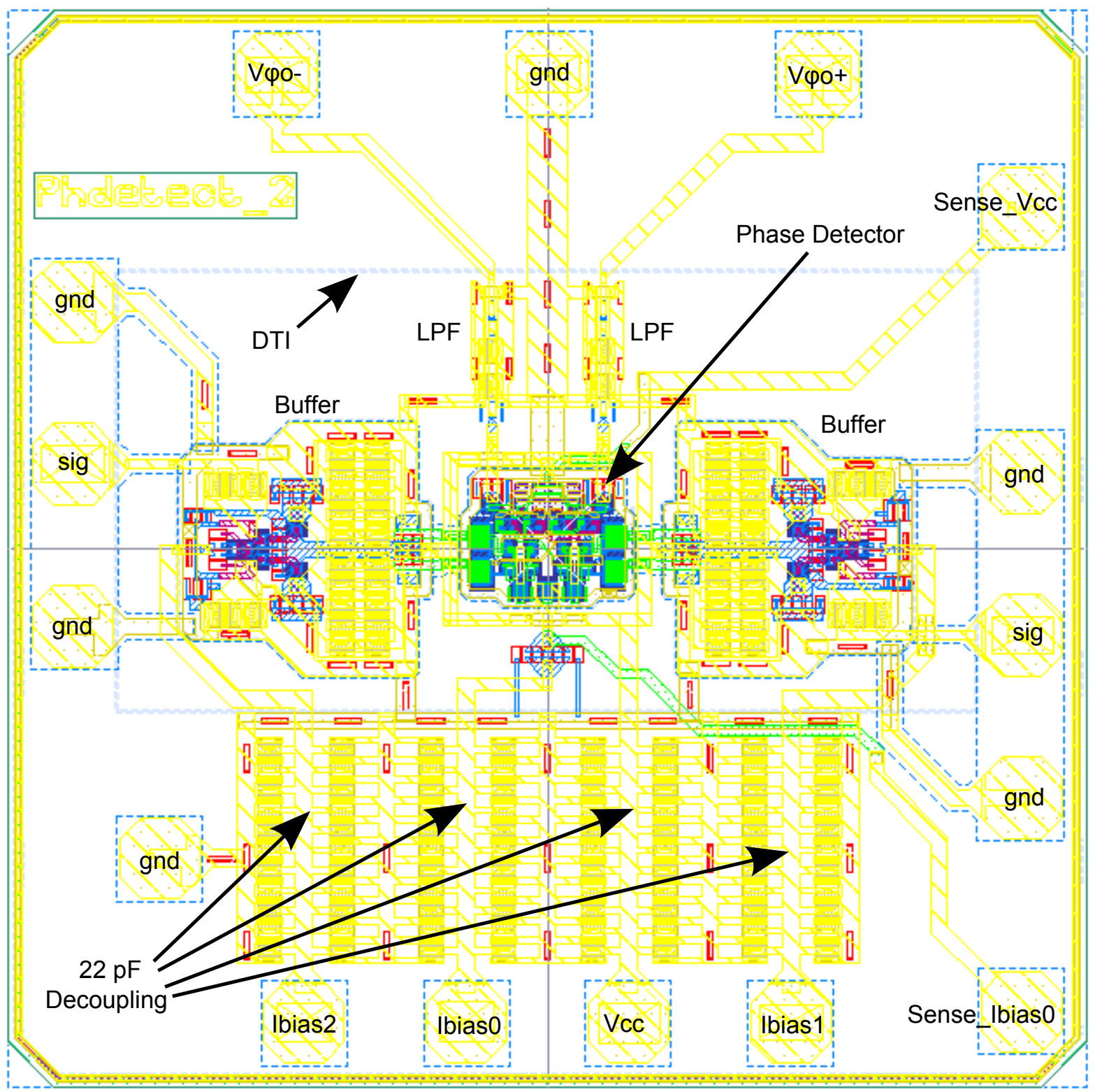

Figure 6.3: Differential Phase Detector (Phdetect_2) Test Device Layout

A phase detector test card was designed and fabricated to provide a test platform for the Phdetect_1 and Phdetect_2 Devices Under Test (DUT), see Appendix C.1 for PCB schematic, layout and BOM. A single DUT was mounted on a phase detector test card ground pad using conductive epoxy so the the high speed signals (i.e., gnd/sig/gnd) could be probed and the remaining low frequency signals could be wire 
bonded, see Figure 6.3. This PCB also included four adjustable Low Drop-Out (LDO) linear voltage regulators and filter circuits to supply Ibias $0 / 1 / 2$ and $V_{C C}$ so they could be controlled independently. These regulators together with LPF components provide attenuation of supply noise from less than $100 \mathrm{~Hz}$ to $1.0 \mathrm{GHz}$. Attenuation of supply noise above $1.0 \mathrm{GHz}$ is provided by the inductance of the bond wires (i.e., typically $1.0 \mathrm{nH} / \mathrm{mm} ;>3.0 \mathrm{~mm}$ or $\approx 3.0 \mathrm{nH})$ that connect these chip pads to pads on the PCB. The LDO regulators are supplied by a $+4.5 V d c$ battery; therefore, any relevant noise on this supply line will originate from the SXOR phase detector switching. The outputs of Phdetect_1 and Phdetect_2, $V \phi o+/-$, are filtered to generate outputs VC_OUT and VC+_OUT. The voltage of these outputs is measured using two Agilent ${ }^{\circledR}$ 34440A 6 1/2 digit Digital Volt Meters (DVM). Figure 6.4 is a functional diagram of the test set-up used to control the phase inputs and measure the corresponding voltage outputs over phase and frequency. 


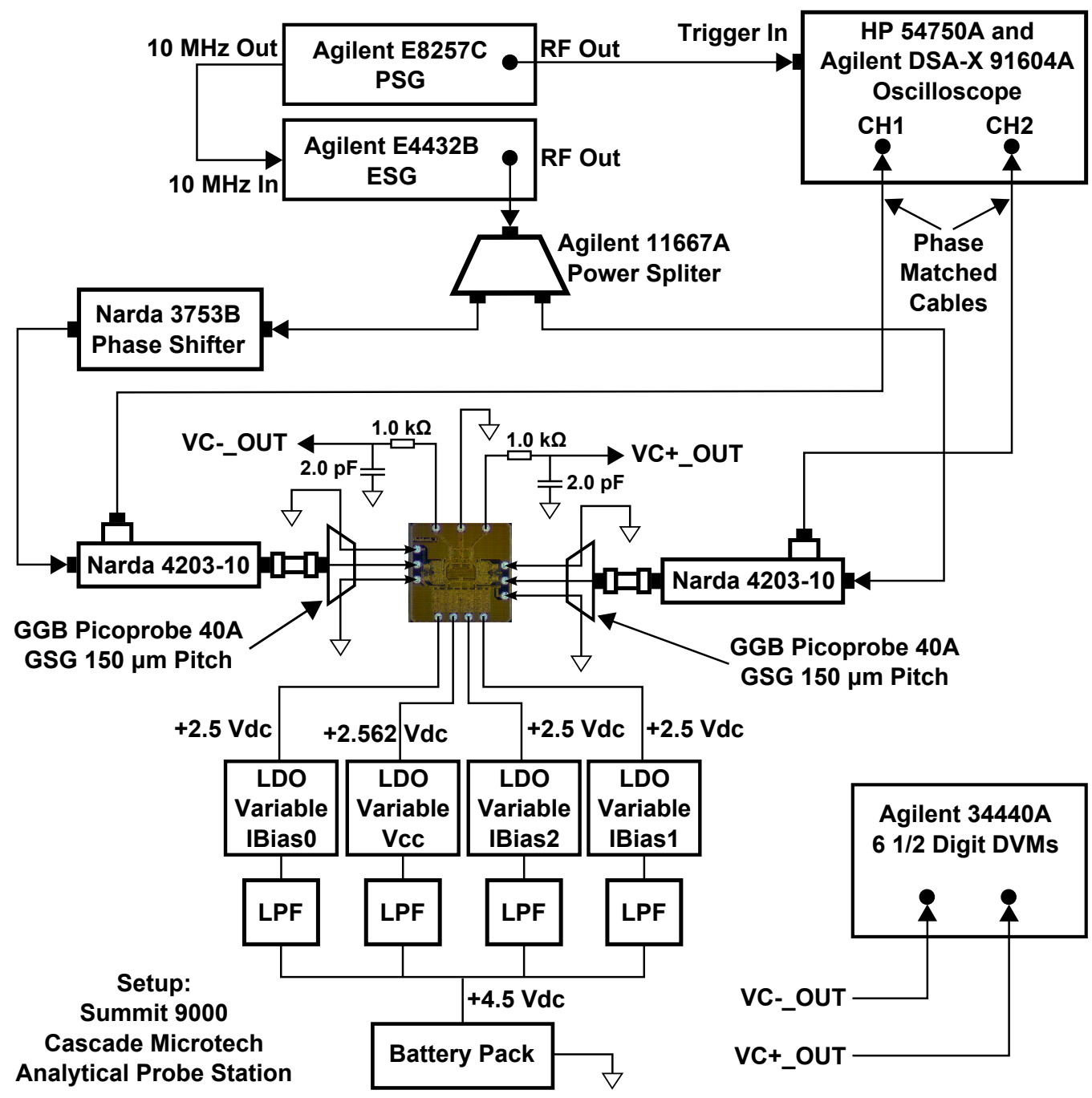

Figure 6.4: Differential Phase Detector Lab Test Set-up

In Figure 6.4 an Agilent E4432B ESG is used to generate a CW tone (RF Out), which is split by an Agilent 11667A Resistive Power Splitter $\left(I L=7 \mathrm{~dB} ; \pm 3^{\circ}\right)$. One branch of this split signal is connected to a Narda ${ }^{\circledR} 4203-1010 d B$ Directional Coupler through a Narda 3753B Phase Shifter $\left(3.5 \mathrm{GHz}\right.$ to $\left.12.4 \mathrm{GHz} \pm 0.5^{\circ}\right)$ and the other branch is connected directly to another Narda 4203-10 $10 d B$ Directional Coupler. The outputs of these Directional Couplers is connected to 40-GHz GSG probes through SMA male-to-male adaptors. The GSG probes supply CW tones to each of 
the inputs of the DUT. This provides controllable phase $\Delta$ input signals at frequencies up to $\approx 12.0 \mathrm{GHz}$. The $10-\mathrm{dB}$ down Directional Coupler outputs are connected to two channels of a 16-GHz Agilent Infiniium DSA-X 91604A Digital Signal Analyzer (i.e., high speed oscilloscope) to measure the phase difference between the resulting CW tones fed to the DUT. These connections are made using cables that are phase matched over temperature and torsion from $100 \mathrm{MHz}$ to $18.0 \mathrm{GHz}$. The stability of these matched cables greatly improves out ability to measure phase increments of $1^{\circ}$ at $12 \mathrm{GHz}$ (i.e., $231.5 \mathrm{fs}$ ) for a long enough duration for the measurement SD to fall below $\approx 80$ fs. An $\mathrm{HP}^{\circledR}$ 54750A 50-GHz Digitizing Oscilloscope was also used to verify these test results.

Eight phase detector test cards were assembled - boards one to four included the version one phase detector die (Phdetect_1, Figure 4.10), boards five to eight included the version two phase detector die (Phdetect_2, Figure 4.11). Table 6.1 lists the voltage setting and the resulting current consumption and power dissipation for each board. Unfortunately, the die probe pads on boards five and eight became contaminated at some point during assembly and could not be probed; therefore, results for these boards were not included in the table below. 
Table 6.1: SXOR Test Card Voltage, Current and Power Dissipation

\begin{tabular}{|r|c|c|c|c|c|c|}
\hline \multicolumn{1}{|c|}{ Parameter } & Card 1 & Card 2 & Card 3 & Card 4 & Card 6 & Card 7 \\
\hline \hline Vcc (V) & 2.5697 & 2.5686 & 2.5705 & 2.5680 & 2.5613 & 2.5683 \\
\hline Vbias0 (V) & 2.5014 & 2.5038 & 2.5015 & 2.5085 & 2.5084 & 2.5060 \\
\hline Vbias1 (V) & 2.5018 & 2.5027 & 2.5034 & 2.5094 & 2.5026 & 2.5051 \\
\hline Vbias2 (V) & 2.5020 & 2.5028 & 2.5035 & 2.5095 & 2.5026 & 2.5053 \\
\hline Sense_Vcc (V) & - & - & - & - & 2.5019 & 2.5020 \\
\hline Sense_Ibias0 (V) & - & - & - & - & 0.7864 & 0.7807 \\
\hline Icc (mA) & 120.6 & 120.4 & 124.1 & 120.6 & 125.6 & 126.8 \\
\hline Ibias0 (mA) & 17.38 & 17.23 & 17.79 & 17.41 & 17.89 & 18.13 \\
\hline Ibias1 (mA) & 7.128 & 7.010 & 7.257 & 7.089 & 7.241 & 7.336 \\
\hline Ibias2 (mA) & 7.083 & 6.990 & 7.260 & 7.048 & 7.267 & 7.323 \\
\hline Power (mW) & 388.9 & 387.4 & 399.8 & 388.9 & 402.9 & 407.8 \\
\hline
\end{tabular}

The Vcc voltage of boards six and seven was adjusted so that the Sense_Vcc voltage was approximately $2.50 \mathrm{~V}$. The Vcc voltage of boards one through four was then set to approximately the same value as the die on these boards did not have Sense_Vcc signals. Sense_Ibias0 voltage levels were verified to be close to the simulation values. The current consumption of Phdetect_2 die is marginally larger than the current consumption of Phdetect_1 die, which may be due to lower path loss in the more compact Phdetect_2 layout.

\subsection{SXOR Phase Detector Test Set-up Calibration}

In order to take accurate measurements using the test set-up of Figure 6.4 we must first determine the propagation delays associated with the test set-up equipment and cables so they can be de-embedded from the measurement stimulus (i.e., the input phase $\Delta$ ). This calibration was carried out using both direct and indirect methods 
described in the following two subsections.

\subsubsection{Direct Test Set-up Calibration}

The test set-up of Figure 6.4 can be used to directly determine the correction that needs to be applied to the input phase difference stimulus. When the SXOR phase detector input phase difference is $90^{\circ}$ the phase detector output voltages VC-_OUT and $\mathrm{VC}+$ _OUT will be equal if operating correctly. Therefore, the differential output voltage should be VC+_OUT - VC-_OUT $=0 \mathrm{~V}$. The test set-up calibration procedure is as follows:

1. Power-up the test equipment for at least 45 minutes before it will be used.

2. Set the E4433B ESG to generate a $12.0 \mathrm{GHz} \mathrm{CW}$ tone. This frequency is limited by the tuning resolution of the Narda 3753B phase adjuster.

3. Set the E4433B ESG CW tone output level to $11.4 \mathrm{dBm}$. This will produce signal levels of $\approx 600 \mathrm{mV}$ when measured at the output port of either Narda 4203-10 directional coupler using the DSA-X 91604A oscilloscope (50 $\Omega$ termination). These output ports are connected to the probes through SMA adapters in Figure 6.4. This level also ensures that a large enough CW tone can be measured at the $-10 \mathrm{~dB}$ coupled ports.

4. Re-connect the test set-up as in Figure 6.4 and power up the DUT.

5. Connect the 34440A DVMs to VC-_OUT and VC+_OUT and adjust the 3753B phase shifter until these outputs are equal.

6. Measure the input phase difference at the zero-crossing points using the DSA$\mathrm{X}$ 91604A oscilloscope. This phase correction factor is the difference between 
the measured input phase difference and the $90^{\circ}$ phase difference that is being applied to the DUT.

This correction factor was used to adjust the input phase difference for all the phase detector measurements included in this section.

This calibration method is simple and direct, and was carried out at the beginning of every test run to minimize drift error during the experiment period. However, this calibration method assumes that the DUT is functioning without any phase offset problems. The indirect test set-up calibration described in the next subsection will mitigate this issue.

\subsubsection{Indirect Test Set-up Calibration}

The set-up illustrated in Figure 6.5 shows how this test set-up calibration procedure was carried out. 
Oscilloscope Measurement 1 = Time Channel 1 - Time Channel 2

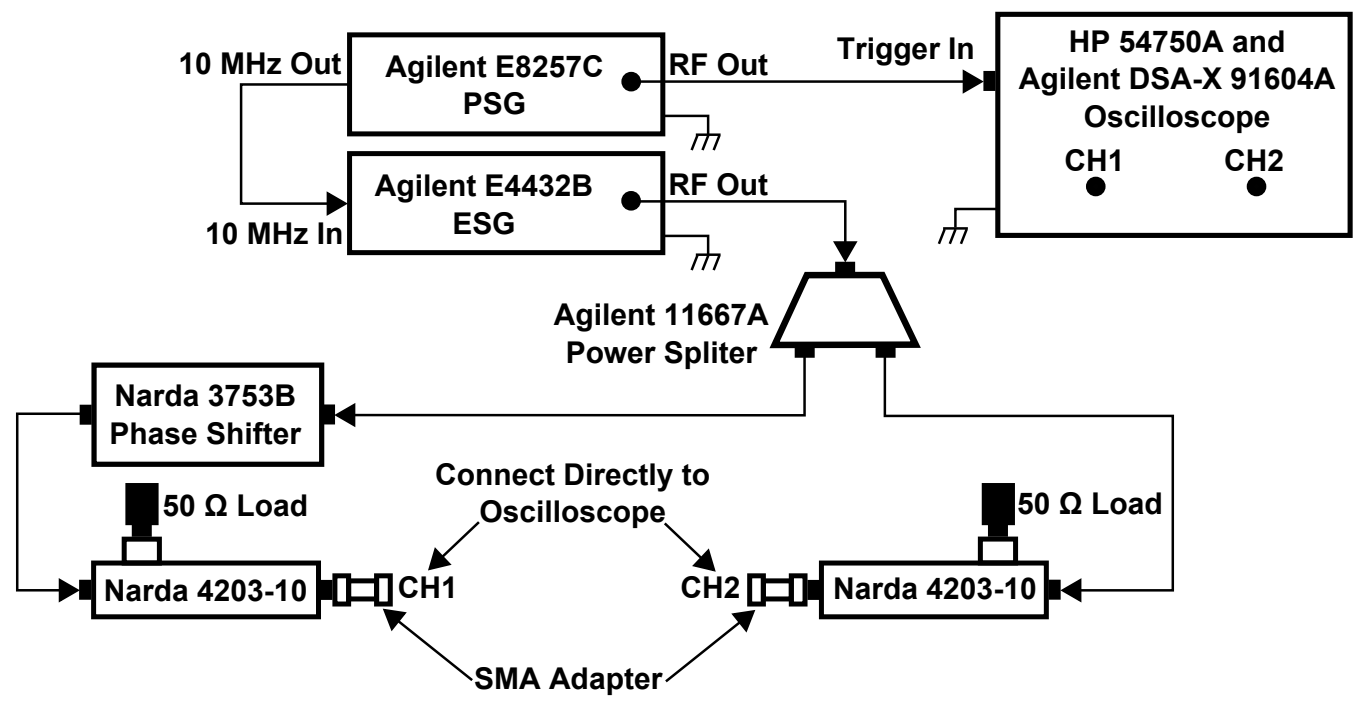

Oscilloscope Measurement 2 = Time Channel 1 - Time Channel 2

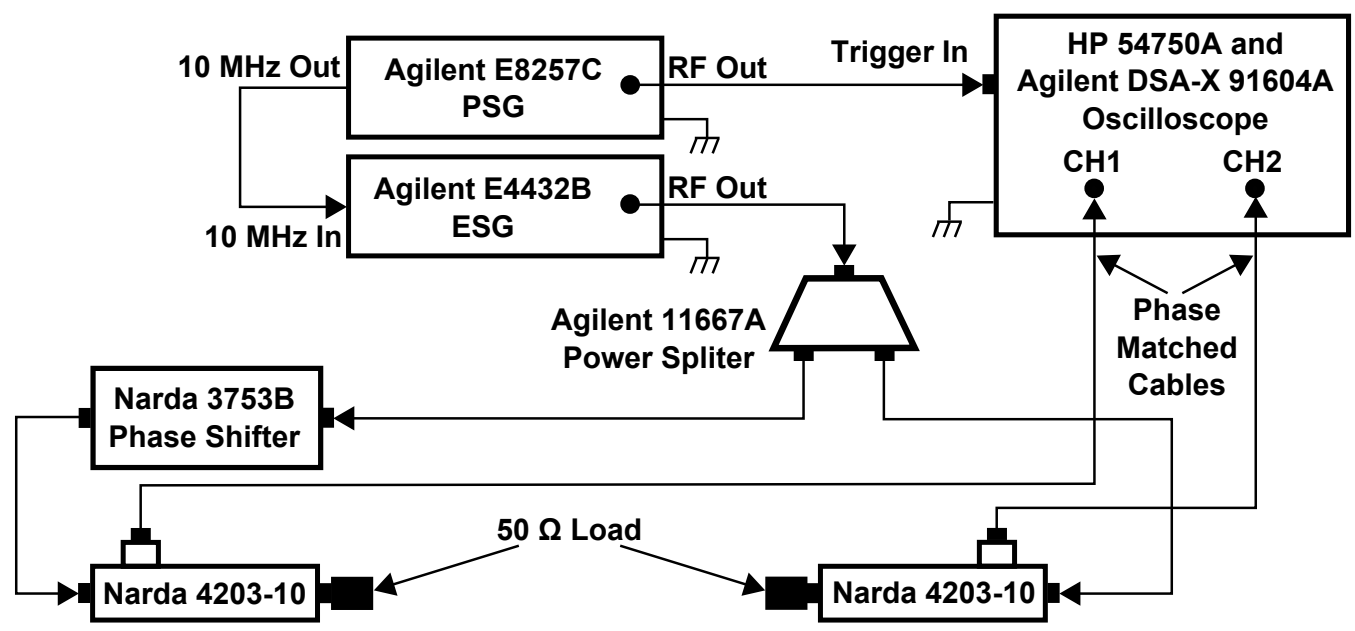

Figure 6.5: Test Cable Delay De-Embedding Set-up

The first three steps in the Direct Test Set-up Calibration procedure were carried out. At this point the phase difference between the signals applied to the DUT was measured and recorded with the DSA-X 91604A oscilloscope for a nominal phase at a frequency of $12 \mathrm{GHz}$. That is, at the directional coupler outputs with the 10- $d B$ coupled outputs terminated in $50 \Omega$ loads. Next, the directional coupler outputs were terminated in $50 \Omega$ loads and the phase difference between the 10- $d B$ coupled outputs 
through matched cables was measured with the DSA-X 91604A oscilloscope at $12 \mathrm{GHz}$ for the same phase setting. The first measurement value was then subtracted from the second measurement value to determine the phase delay in degrees that was used to adjust the input phase stimulus. This procedure moved the measurement plane to the input of the probes.

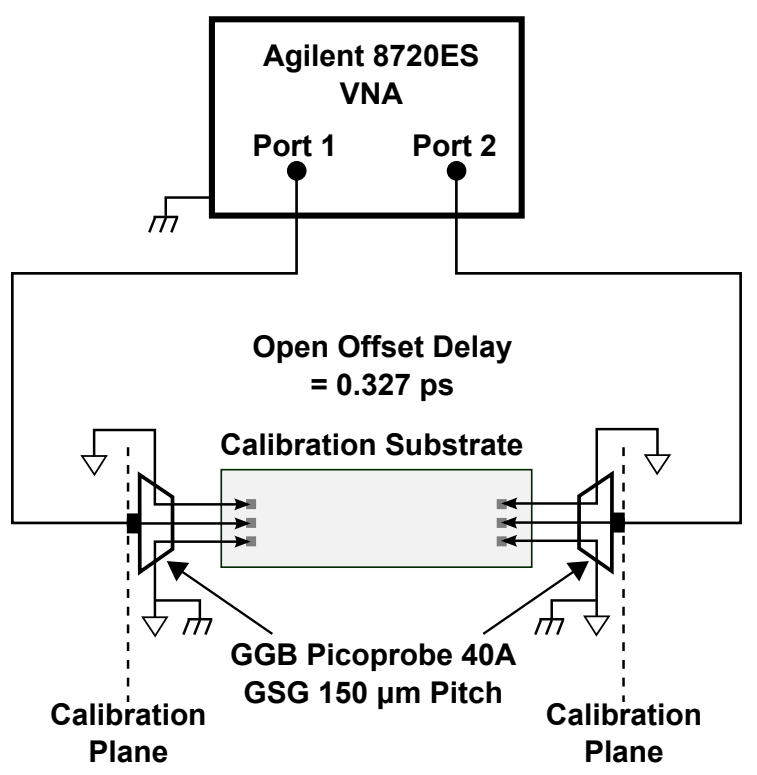

Figure 6.6: Probe Delay De-Embedding Set-up

The delay through the probes was determined using an Agilent 8720ES Vector Network Analyzer (VNA). First, single port VNA calibrations (i.e., s11 and s22) at $12 \mathrm{GHz} \pm 100 \mathrm{MHz}$ were carried out to the ends of the VNA cables that will connect to the probes (i.e., Calibration Plane of Figure 6.6). Next, the VNA cables were connected to the GSG probes and the probes were landed on the open pads of a Calibration Substrate. The s11 and s22 delay was measured and recorded.

This measurement is the round-trip delay from the calibration plane to the probe tips, across the open offset delay (i.e., $0.327 \mathrm{ps}$ ) and back from the probe tip to the calibration plane. The delay from calibration plane to probe tip for GSG probe V92, with measured delay $=182.1 \mathrm{ps}$, is give by equation (6.1). 


$$
(182.1 / 2)-0.327=90.723 p s
$$

The delay from calibration plane to probe tip for GSG probe W31, with measured delay $=178.8 \mathrm{ps}$, is give by equation (6.2).

$$
(178.8 / 2)-0.327=89.073 p s
$$

The difference between these two delays, $90.723-89.073=1.65 \mathrm{ps}$, was used to adjust the input stimulus phase delay value.

The input stimulus phase delay values determine using the Indirect Test Setup calibration procedure were found to be consistent with the input stimulus phase delay values found using the Direct Test Set-up Calibration procedure. This showed that there were no significant input phase offsets between the two differential phase detector inputs. Further, the Direct Test Set-up Calibration procedure was used to generate the test results presented in this document.

\subsection{SXOR Phase Detector Test Results}

Testing was focused on boards five to eight because significant improvements had been made to the Phdetect_2 die (i.e., improved layout, phase detector sense line on VCC and Ibias0 and additional ground pads). Board four, including the Phdetect_1, was also tested to verify the operation of this device.

The ECL outputs at Out-/+ of Figure 6.7 will have an offset error that is approximately equal to the error in $V_{C C}$ from its nominal voltage. This error can be removed by subtracting Out_Avg-from Out_Avg+ to get a differential voltage, V $\phi$ Measured. 


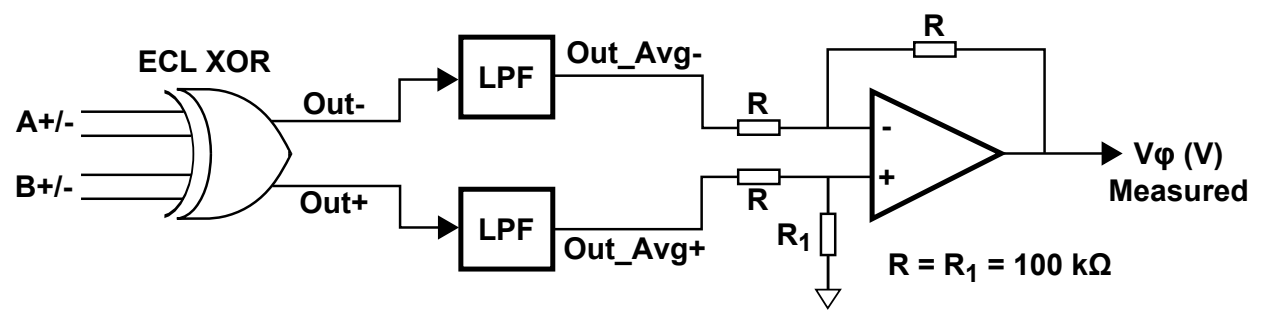

Figure 6.7: Op Amp Removal of $V_{C C}$ Error

The results plotted in Figures 6.8 and 6.9 and listed in Tables 6.2 and 6.3 were obtained by measuring the steady state voltage at Out_Avg- $/+$ w.r.t. ground and subtracting the results. Figure 6.7 shows how this function could be implemented with a CMOS Op Amp. R1 can be used to remove any significant de offset that exits in the Op Amp. 


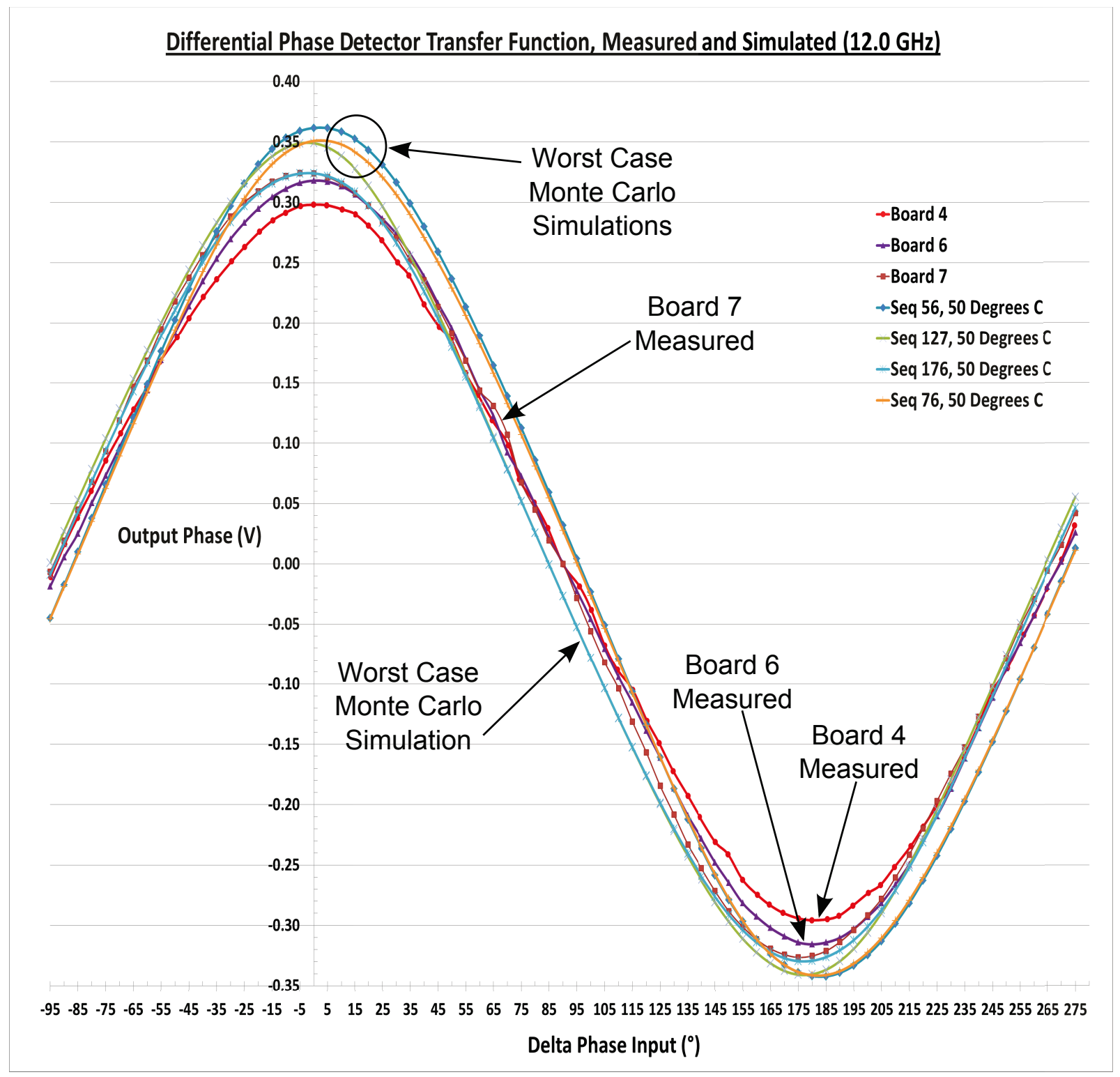

Figure 6.8: Phase Detector Transfer Function - 12.0 GHz, Measured

Figure 6.8 shows the transfer function measurements of Boards 4, 6 and 7 for input phase steps of $5^{\circ}$. These results compare well with the ideal transfer function shown in Figure 4.2 except for the Output Phase extremes. The curves are monotonic for input phase steps of $5^{\circ}$.

Also, plotted are Monte Carlo simulation results that were carried out on the extracted version of the tested circuit of Figure 6.3. These Monte Carlo results 
(i.e., over process and mismatch) were worst case of 200 runs at $50^{\circ} \mathrm{C}$. The $50^{\circ} \mathrm{C}$ temperature value was estimated by matching nominal simulation results at elevated temperatures with the measured results. Here we see that for input phase differences around $45^{\circ}$ and $90^{\circ}$ the test results fall between the Monte Carlo simulations.

\section{Differential Phase Detector Output, Measured and Simulated (12.0 GHz)}

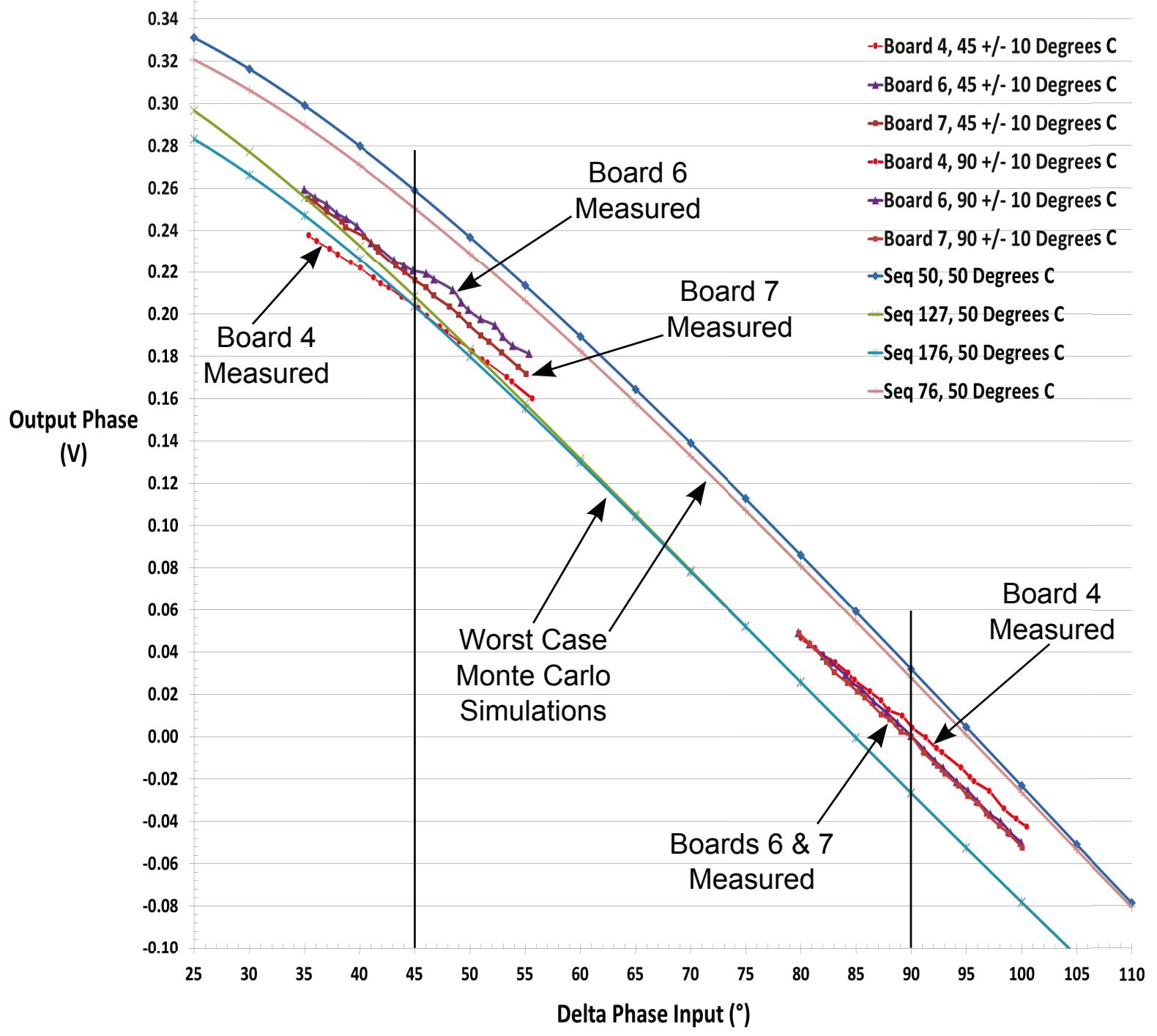

Figure 6.9: Phase Detector Transfer $45^{\circ} \pm 10^{\circ}$ and $90^{\circ} \pm 10^{\circ}-12.0 \mathrm{GHz}$, Measured

Figure 6.9 shows the transfer function measurements of Boards 4, 6 and 7 for input phase steps of $1^{\circ}$ over the ranges of $45^{\circ} \pm 10^{\circ}$ and $90^{\circ} \pm 10^{\circ}$. The curves are 
monotonic for input phase steps of $1^{\circ}$. The variation in monotonicity shown in this plot is mostly due to the phase tuner. The Narda 3753B is a mechanical tuner that is adjusted by hand. It has problems with repeatability and resolution at $12 \mathrm{GHz}$.

The test results for the version two phase detectors (i.e., Phdetect_2) on Boards 6 and 7 fall within the Monte Carlo simulations. The test results for the version one phase detector, Board 4 , around $45^{\circ}$ fall below the lower Monte Carlo simulation result. This excursion is not surprising as the Monte Carlo simulations were carried out on the extracted version two phase detector.

Table 6.2 lists the data plotted in Figure 6.9 for the range of $45^{\circ} \pm 10^{\circ}$. We can see from the Input Phase $\Delta$ values the difficulty in adjusting the mechanical phase tuner. The important results of this table are the slope values $4.2 \mathrm{mV}$ for Board 7, $3.8 \mathrm{mV}$ for Board 6 and $3.8 \mathrm{mV}$ for Board 4. These values indicate the average differential output voltage for an input phase step of $1^{\circ}$.

Table 6.3 lists the data plotted in Figure 6.9 for the range of $90^{\circ} \pm 10^{\circ}$. Here the slope values are $5.0 \mathrm{mV}$ for Board 7, $4.9 \mathrm{mV}$ for Board 6 and $4.4 \mathrm{mV}$ for Board 4. These values indicate the average differential output voltage for an input phase step of $1^{\circ}$. It should be noted that there is approximately $1 \mathrm{mV}$ difference in voltage between these two ranges. This is likely due to the improved linearity of the phase measurements taken around $90^{\circ}$ versus those taken around $45^{\circ}$. 
Table 6.2: Summary of Measured Results for $45^{\circ} \pm 10^{\circ}-12.0 \mathrm{GHz}$, Measured

\begin{tabular}{|c|c|c|c|c|c|}
\hline \multicolumn{2}{|c|}{ Board $7\left(45^{\circ} \pm 10^{\circ}\right)$} & \multicolumn{2}{|c|}{ Board $6\left(45^{\circ} \pm 10^{\circ}\right)$} & \multicolumn{2}{|c|}{ Board $4\left(45^{\circ} \pm 10^{\circ}\right)$} \\
\hline Input & $\mathbf{V} \phi(\mathbf{V})$ & Input & $\mathbf{V} \phi(\mathbf{V})$ & Input & $\mathbf{V} \phi(\mathbf{V})$ \\
\hline Phase $\Delta\left(^{\circ}\right)$ & Measured & Phase $\Delta\left(^{\circ}\right)$ & Measured & Phase $\Delta\left(^{\circ}\right)$ & Measured \\
\hline 35.28 & 0.2551 & 34.93 & 0.2594 & 35.33 & 0.2376 \\
\hline 35.80 & 0.2539 & 35.93 & 0.2555 & 36.05 & 0.2350 \\
\hline 36.98 & 0.2490 & 36.96 & 0.2524 & 37.23 & 0.2313 \\
\hline 38.33 & 0.2443 & 37.91 & 0.2481 & 37.98 & 0.2286 \\
\hline 38.68 & 0.2417 & 38.75 & 0.2456 & 39.15 & 0.2247 \\
\hline 40.35 & 0.2371 & 39.73 & 0.2419 & 39.99 & 0.2218 \\
\hline 41.46 & 0.2321 & 41.06 & 0.2341 & 41.25 & 0.2172 \\
\hline 41.71 & 0.2301 & 41.79 & 0.2316 & 41.90 & 0.2145 \\
\hline 43.28 & 0.2230 & 43.14 & 0.2258 & 42.65 & 0.2125 \\
\hline 44.08 & 0.2199 & 44.04 & 0.2231 & 43.80 & 0.2081 \\
\hline 45.02 & 0.2160 & 44.85 & 0.2207 & 45.29 & 0.2027 \\
\hline 46.00 & 0.2126 & 46.02 & 0.2192 & 46.05 & 0.1991 \\
\hline 46.72 & 0.2087 & 46.75 & 0.2165 & 47.25 & 0.1939 \\
\hline 48.14 & 0.2035 & 48.43 & 0.2113 & 47.86 & 0.1914 \\
\hline 48.97 & 0.1997 & 49.22 & 0.2055 & 49.02 & 0.1870 \\
\hline 49.92 & 0.1947 & 49.85 & 0.2019 & 50.20 & 0.1823 \\
\hline 50.97 & 0.1898 & 50.97 & 0.1976 & 51.10 & 0.1784 \\
\hline 51.72 & 0.1869 & 52.25 & 0.1945 & 51.55 & 0.1770 \\
\hline 52.85 & 0.1818 & 52.96 & 0.1893 & 53.32 & 0.1702 \\
\hline 54.36 & 0.1750 & 53.86 & 0.1850 & 53.78 & 0.1679 \\
\hline 55.07 & 0.1717 & 55.34 & 0.1812 & 55.62 & 0.1600 \\
\hline Range (V) & 0.0834 & - & 0.0782 & - & 0.0776 \\
\hline Slope $\left(\mathrm{V} /{ }^{\circ}\right)$ & 0.0042 & - & 0.0038 & - & 0.0038 \\
\hline
\end{tabular}


Table 6.3: Summary of Measured Results for $90^{\circ} \pm 10^{\circ}-12.0 \mathrm{GHz}$, Measured

\begin{tabular}{|c|c|c|c|c|c|}
\hline \multicolumn{2}{|c|}{ Board $7\left(90^{\circ} \pm 10^{\circ}\right)$} & \multicolumn{2}{|c|}{ Board $6\left(90^{\circ} \pm 10^{\circ}\right)$} & \multicolumn{2}{|c|}{ Board $4\left(90^{\circ} \pm 10^{\circ}\right)$} \\
\hline Input & $\mathbf{V} \phi(\mathbf{V})$ & Input & $\mathbf{V} \phi(\mathbf{V})$ & Input & $\mathbf{V} \phi(\mathbf{V})$ \\
\hline Phase $\Delta\left(^{\circ}\right)$ & Measured & Phase $\Delta\left(^{\circ}\right)$ & Measured & Phase $\Delta\left(^{\circ}\right)$ & Measured \\
\hline 79.82 & 0.0489 & 79.80 & 0.0491 & 80.01 & 0.0464 \\
\hline 80.81 & 0.0438 & 80.79 & 0.0438 & 81.27 & 0.0 .0417 \\
\hline 82.32 & 0.0353 & 82.06 & 0.0381 & 81.96 & 0.0386 \\
\hline 83.03 & 0.0304 & 82.71 & 0.0351 & 83.09 & 0.0350 \\
\hline 84.23 & 0.0254 & 84.04 & 0.0291 & 84.24 & 0.0301 \\
\hline 85.12 & 0.0213 & 84.47 & 0.0260 & 84.84 & 0.0267 \\
\hline 85.80 & 0.0185 & 85.52 & 0.0223 & 86.23 & 0.0213 \\
\hline 87.29 & 0.0105 & 86.58 & 0.0166 & 87.24 & 0.0 .0170 \\
\hline 88.06 & 0.0081 & 87.78 & 0.0113 & 87.94 & 0.0126 \\
\hline 89.05 & 0.0024 & 88.75 & 0.0065 & 89.15 & 0.0098 \\
\hline 90.00 & 0 & 90.00 & 0.0003 & 90.11 & 0.0042 \\
\hline 91.13 & -0.0076 & 91.21 & -0.0063 & 91.29 & -0.0005 \\
\hline 92.37 & -0.0136 & 92.15 & -0.0115 & 92.26 & -0.0055 \\
\hline 93.06 & -0.0175 & 92.88 & -0.0149 & 92.78 & -0.0075 \\
\hline 94.27 & -0.0230 & 94.12 & -0.0213 & 94.49 & -0.0147 \\
\hline 95.10 & -0.0279 & 95.14 & -0.0257 & 95.29 & -0.0192 \\
\hline 96.04 & -0.0314 & 95.97 & -0.0304 & 95.67 & -0.0212 \\
\hline 96.82 & -0.0364 & 97.17 & -0.0367 & 97.05 & -0.0258 \\
\hline 97.96 & -0.0420 & 98.11 & -0.0402 & 98.36 & -0.0340 \\
\hline 98.76 & -0.0455 & 99.01 & -0.0454 & 99.47 & -0.0389 \\
\hline 100.05 & -0.0523 & 99.96 & -0.0500 & 100.45 & -0.0426 \\
\hline Range (V) & 0.1012 & - & 0.0991 & - & 0.0890 \\
\hline Slope $\left(\mathrm{V} /{ }^{\circ}\right)$ & 0.0050 & - & 0.0049 & - & 0.0044 \\
\hline
\end{tabular}




\subsection{Discussion of Results - SXOR Phase Detector}

Simple Symmetrical XOR (SXOR) phase detectors were designed, fabricated in SiGe $\mathrm{BiCMOS}$ and tested to resolve $1^{\circ}$ of phase error over $45^{\circ} \pm 10^{\circ}$ and $90^{\circ} \pm 10^{\circ}$ up to $12 \mathrm{GHz}$. This $12 \mathrm{GHz}$ limit is the maximum operating frequency of the test set-up (i.e., limited by the Narda 3753B Phase Shifter), not the maximum operating frequency of the DUT. The following was also demonstrated:

1. The measured SXOR transfer functions of multiple devices show good agreement with the ideal transfer function.

2. The measured SXOR transfer functions of multiple devices were monotonic for input phase steps of $1^{\circ}$ over the ranges of $45^{\circ} \pm 10^{\circ}$ and $90^{\circ} \pm 10^{\circ}$.

3. The measured SXOR phase detector average output voltage change for $1^{\circ}$ input phase steps over the ranges of $45^{\circ} \pm 10^{\circ}$ and $90^{\circ} \pm 10^{\circ}$ was $\approx 4 \mathrm{mV}$ and $\approx 5 \mathrm{mV}$. These output voltages were consistent over these ranges.

4. The measured SXOR transfer functions of multiple devices show good agreement with simulation at $50^{\circ} \mathrm{C}$. That is, these results are between the worst case extracted simulation Monte Carlo curves.

5. The differential transit delay through an XOR circuit can be equalized by using a symmetrical XOR or SXOR architecture.

6. The simple ECL outputs of these phase detectors are very sensitive to Vcc variation in single-ended operation. This problem can be mitigated by using the SXOR outputs differentially.

7. The SXOR phase detector and buffer power dissipation values listed in Table 6.1 are very high. This is because the transistors of these components were biased 
for a current density close to $f_{\mathrm{T}}$, which is equivalent to an Ic $=14$ to $18 \mathrm{~mA}$. The objective here was to demonstrate RTWO phase tuning and the optimization of power dissipation was considered to be beyond the scope of this work.

The following errors were addressed during the testing of the fabricated SXOR phase detector.

\section{Systematic Error}

1. Two test measurement calibration methods (Direct and Indirect) were developed and used to remove phase error in the test set-up. These methods were carried out immediately before every test run and the results were used to calibrate the input phase stimulus.

2. The phase delay difference between the two GSG probes was determined and removed from the input phase stimulus.

3. Cables matched over torsion and temperature up to $18 \mathrm{GHz}$ were used to measure phase difference. This cables have a loss of $\approx 1.4 \mathrm{~dB}$ at $18 \mathrm{GHz}$ and significantly improved measurement stability and repeatability.

4. Care had to taken to ensure the GSG probes were landed solidly on the die pads. That is, so that normal vibration in the test area did not move the probes on the pads. Probe to pad movement caused the phase detector output voltage to jump significantly.

\section{Random Error}

1. The manual phase adjustment mechanism of the Narda phase adjuster was not smooth or continuous. Generally, the phase would change initially in the 
opposite direction of adjustment and then jump to a new phase position in the intended direction of adjustment. A significant amount of fine tuning was required for each input phase $\Delta$. Unfortunately, no other phase adjusters were available and the purchase a an electronic phase adjuster with adequate range and resolution was prohibitively expensive.

2. The high speed oscilloscope used to measure phase was configured to take a moving average of 256 measurements. After the input phase adjustment was made for each measurement point, the moving average was reset and a phase value was recorded after the SD was stable and less than $80 \mathrm{fs}$.

\section{Drift Error}

1. Test equipment was turned on and allowed to warm up to an operating temperature for at least 45 minutes before each test run.

2. All test runs were carried out without pauses. The objective here was to minimize the effects of room temperature variation and other test equipment drift mechanisms over time.

3. At the beginning of each test the system was calibrated to $90^{\circ}$. Measurements were then taken from this point in the direction of decreasing input phase delta (i.e., $89^{\circ}, 88^{\circ} \ldots$ ). After measurements were take in this direction, measurements were taken in the direction of increasing input phase delta (i.e., $89^{\circ}, 88^{\circ}$ ... ). In the time between these two measurement sequences drift error occurred that can be seen in the test result graphs, Figures 6.8 and 6.9. 


\subsection{RTWO Phase Alignment Test Set-up and Pro- cedure}

Figure 6.10 shows the test set-up used to evaluate the RTWO phase alignment test die. An RTWO tuning test card was designed and fabricated to provide a platform for this DUT, see Appendix C.2 for PCB schematic, layout and BOM. A single DUT was mounted on an RTWO phase alignment test card ground pad using conductive epoxy so the high speed RTWO output signals (i.e., GND/OSC-/OSC+/GND) could be probed, and their amplitude and frequency measured using an HP 8593E spectrum analyzer. This test card supports nine adjustable LDO power supplies, which receive power from an Agilent E3546A DC supply through nine separate power supply filters. These regulators together with LPF components provide attenuation of supply noise from frequencies of less than $100 \mathrm{~Hz}$ to $1.0 \mathrm{GHz}$. Attenuation of supply noise above 1.0 $\mathrm{GHz}$ is provided by the inductance of the bond wires (i.e., typically $1.0 \mathrm{nH} / \mathrm{mm}$; $>3.0 \mathrm{~mm}$ or $\approx 3.0 \mathrm{nH})$ that connect these chip pads to pads on the PCB. The RTWO gain stage amplifiers were supplied directly from an Agilent E3646A power supply through VccOSC as this voltage was adjusted beyond the range of the LDO regulators.

The voltage levels of the filtered single ended SXOR phase detector output signals, VOELLB/VOETL/VOERT/VOEBR, were measured using three Agilent 34401A and one Keithley ${ }^{\circledR} 20106$ 1/2 digit DVM. These signals were also sampled and digitized using the Maxim ${ }^{\circledR}$ MAX126 4-channel, 14-bit ADC of a MAX126 evaluation board. This board provided a $16.0 \mathrm{MHz}$ oscillator, LDO regulators and $2.5 \mathrm{~V}$ voltage reference required by the MAX126. This sets the analogue input voltage range to be $-2.5 \mathrm{~V}$ to $+2.5 \mathrm{~V}$. The input voltage range required in this application is 0 to $+2.5 \mathrm{~V}$; therefore, the most significant bit of the two's complement digital outputs will not be 
used, making the ADC effectively 13-bits.

$$
2.5 /\left(2^{13}-1\right)=305.2 \mu \mathrm{V}
$$

Equation (6.3) shows the resolution of the ADC to be $305.2 \mu \mathrm{V}$. The $\pm 12.0 \mathrm{Vdc}$ required for this card were supplied by an Agilent E3646A DC supply.

The digitized phase detector outputs were converted from $5.0 \mathrm{~V}$ logic to $3.3 \mathrm{~V}$ logic and fed to the hardware implementation of the phase calibration algorithm, which was realized in a Diligent Atlys (Xilinx ${ }^{\circledR}$ ) Spartan-6 FPGA board. The algorithm timer input zero (T0i, Figure 3.10) was connected to a Single-Pole Single-Throw (SPST) switch to allow the algorithm to be reset manually. The algorithm timer input one (T1i, Figure 3.10), which initiates an iteration of the phase alignment algorithm on every low-to-high state change, was connected to a SPST momentary switch to allow single-stepping through RTWO phase correction process. Both these switches were debounced within the algorithm, see Appendix B.2.

After the state of T0i is changed from reset, the phase calibration algorithm will initialize the varactor voltages to be mid range (i.e., $\approx 1.25 V d c$ ) and the Maxim MAX126 ADC is initialized to carry out a four channel conversion sequence at every iteration. After every T1i low-to-high state change the algorithm will carry out four consecutive reads of the MAX126 and uses these digital voltage levels to generate new varactor voltages.

The results of each algorithm iteration are digital representations of newly generated varactor control voltage levels, which are presented to an Analog Devices ${ }^{\circledR}$ AD7305 4-channel, 8-bit DAC mounted on the RTWO tuning test card. This communication occurs over a 12-signal parallel A/D/C (Address/Data/Control) bus that 
cycles through the four digital number to analogue voltage conversions that are generated by every algorithm iteration. The DAC output voltage range is 0 to $+2.5 \mathrm{~V}$, which is determined by the $+2.5 \mathrm{~V}$ reference voltage generated on the Maxim MAX126 ADC card. Equation (6.4) shows the resolution of the ADC to be $9.804 \mathrm{mV}$.

$$
2.5 /\left(2^{8}-1\right)=9.804 m V
$$

The varactor control voltages, VCL/VCT/VCR/VCB, were measured using four Wavetech ${ }^{\circledR} 31 / 2$ digit DVMs. 


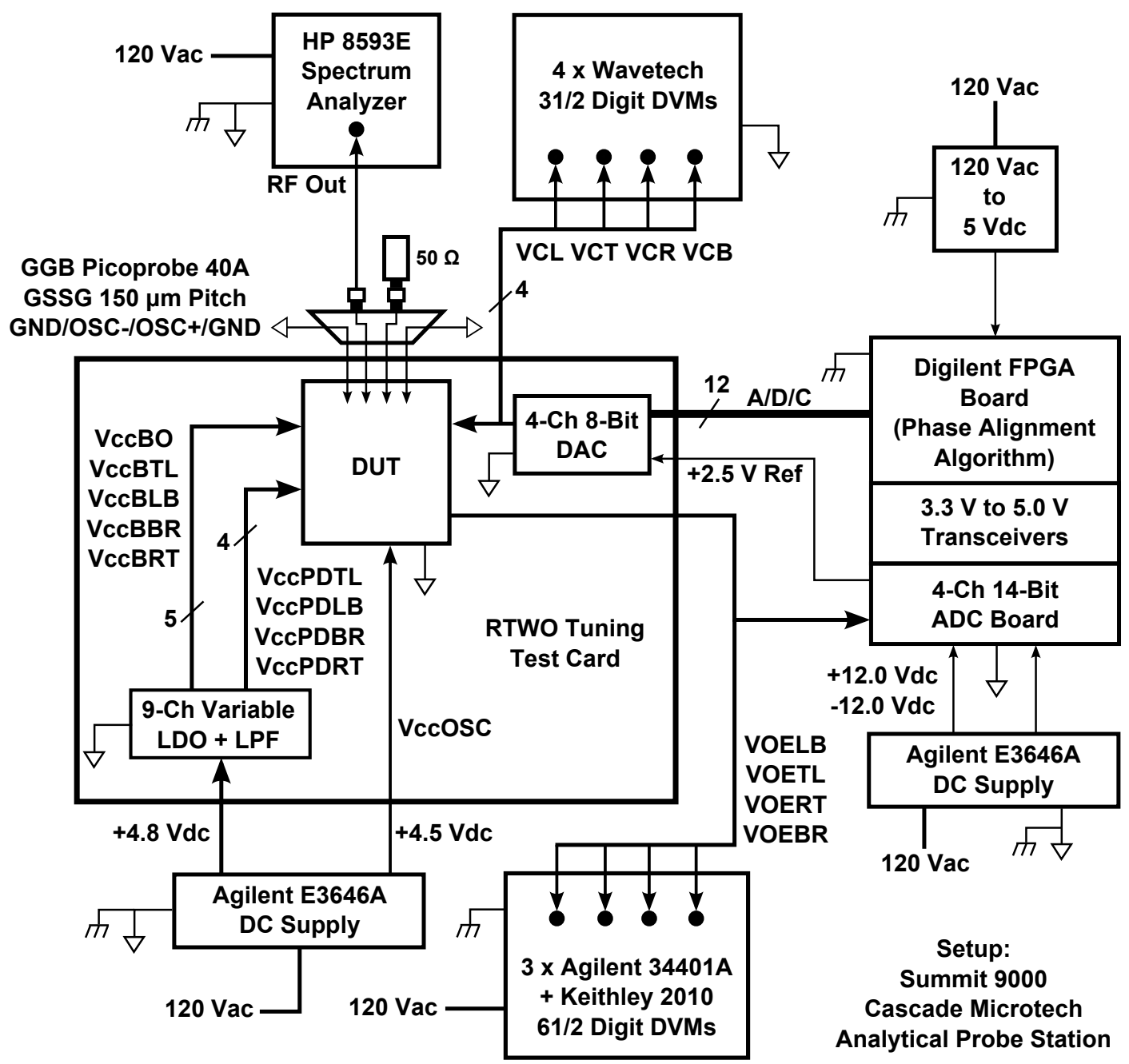

Figure 6.10: Tuneable RTWO Lab Test Set-up

Figure 6.11 is a die photograph of the RTWO (centre) with differential buffers and SXOR phase detectors (at the corners) that is the DUT of Figure 6.10. This RTWO was created by Kimia Ansari who also assembled this layout and submitted it for fabrication. All signals except for the GND/OSC-/OSC+/GND were wire bonded to the RTWO Tuning Test Card (Figures C.5 and C.6). Signals GND/OSC/OSC+/GND were probed as shown in Figure 6.10. 


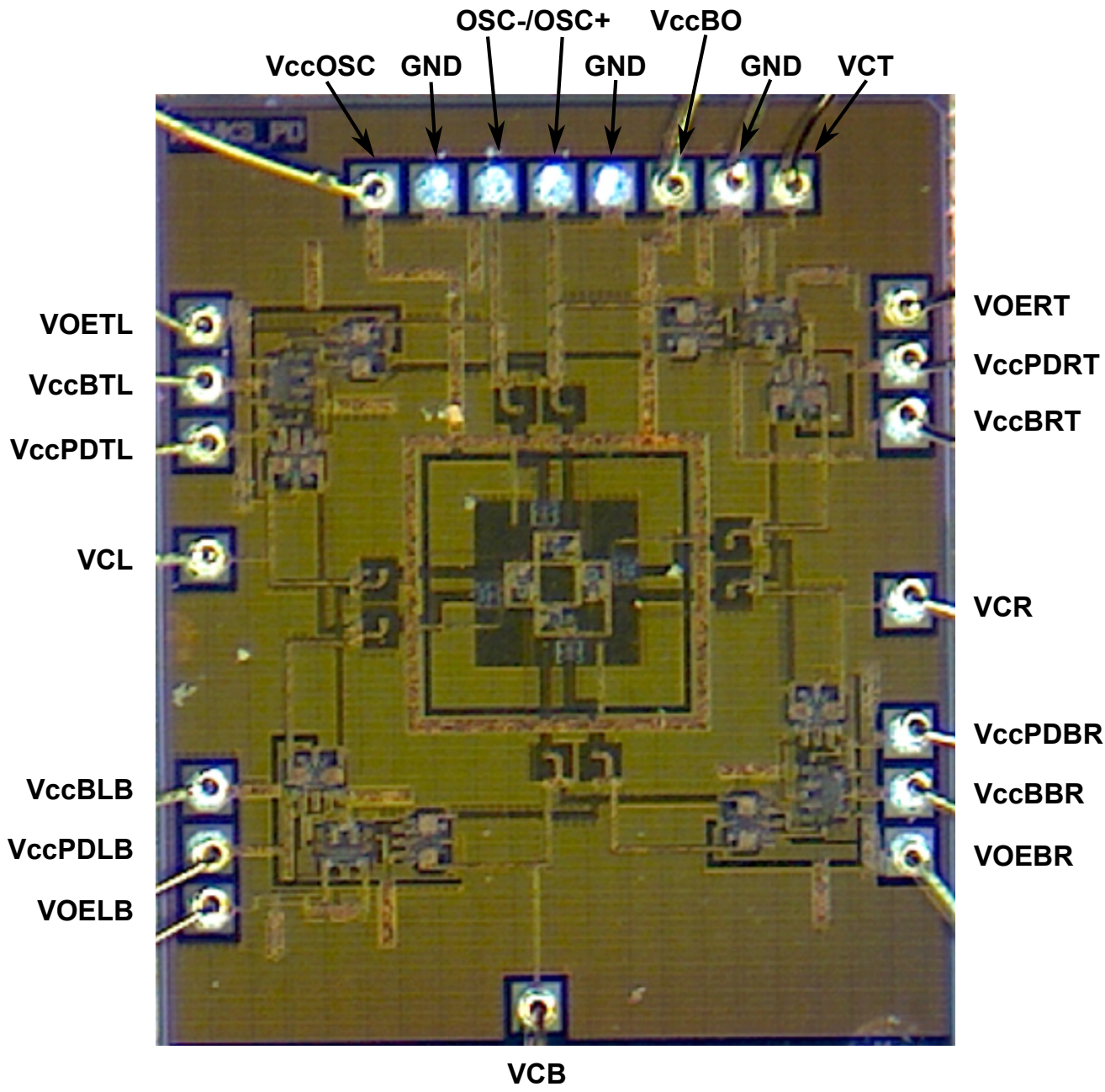

Figure 6.11: RTWO Phase Alignment Test Die (DUT Figure 6.10)

VCL/VCB/VCR/VCT of Figure 6.11 are the varactor control signals for the Left, Bottom, Right and Top sections of the RTWO. VccOSC supplies power to the RTWO amplifiers and $\mathrm{VccBO}$ supplies power to the buffers that tap the oscillator phases directly from the RTWO sections. VOETL/VOELB/VOEBR/VOERT are single ended filtered phase detector output signals from the Top-Left, Left-Bottom, BottomRight and Right-Top corners of the RTWO. VccBTL/VccBLB/VccBBR/VccBRT 
supply power to the differential buffers that drive the inputs of the differential SXOR phase detectors with oscillator phases from the tap buffers. VccPDTL/VccPDLB/VccPDBR/VccPDRT supply the differential SXOR devices at each corner.

Table 6.4 lists the supply voltages, currents and total power dissipation for the two RTWO Tuning Test Cards used to evaluate the RTWO phase alignment test die. Here $V c c B O \approx 2.5 V d c$ and $V c c O S C \approx 4.5 V d c$ were set based on testing done by Kimia Ansari. The differential buffer supply voltages were set to $\approx 2.56 \mathrm{~V}$ based on the phase detector testing results shown in the Vcc row of Table 6.1. The SXOR phase detector supply voltages were adjusted so the filtered outputs would have similar initial values.

Table 6.4: RTWO Tuning Test Card Voltage, Current and Power Dissipation

\begin{tabular}{|r|c|c|r|c|c|}
\hline \multicolumn{1}{|c|}{ Parameter } & Card 1 & Card 2 & Parameter & Card 1 & Card 2 \\
\hline \hline VccBO (V) & 2.489 & 2.505 & IccBO (mA) & 202.4 & 200.3 \\
\hline VccOSC (V) & 4.500 & 4.500 & IccOSC (mA) & 21.0 & 21.0 \\
\hline VccBTL (V) & 2.565 & 2.585 & IccBTL (mA) & 84.1 & 85.1 \\
\hline VccPDTL (V) & 2.437 & 2.423 & IccPDTL (mA) & 53.6 & 53.2 \\
\hline VccBLB (V) & 2.561 & 2.571 & IccBLB (mA) & 82.1 & 81.8 \\
\hline VccPDLB (V) & 2.682 & 2.661 & IccPDLB (mA) & 59.4 & 58.7 \\
\hline VccBBR (V) & 2.559 & 2.569 & IccBBR (mA) & 83.2 & 83.3 \\
\hline VccPDBR (V) & 2.650 & 2.565 & IccPDBR (mA) & 58.9 & 56.1 \\
\hline VccPDRT (V) & 2.888 & 2.640 & IccPDRT (mA) & 115.2 & 100.0 \\
\hline VccBRT (V) & 2.683 & 2.571 & IccBRT (mA) & 46.0 & 43.0 \\
\hline \multicolumn{7}{|r}{} & & Power (W) & 2.139 & 2.044 \\
\hline
\end{tabular}




\subsection{RTWO Phase Alignment Test Results}

The test set-up of Figure 6.10 with the DUT of Figure 6.11 were used to generate the results of Figures 6.12 to 6.16 .

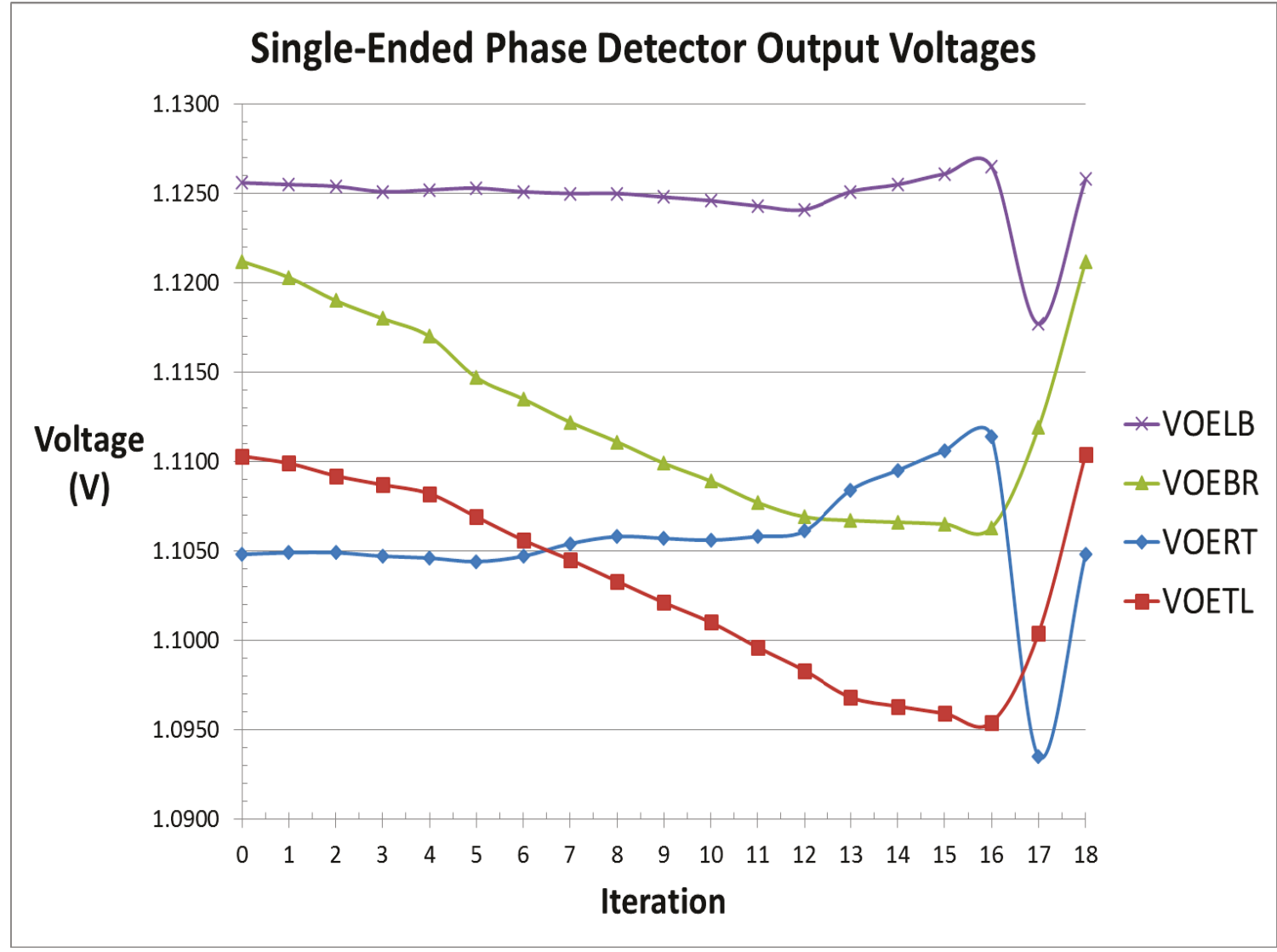

Figure 6.12: Test Board 1 Phase Detector Output Signals

Figure 6.12 shows the single-ended SXOR phase detector output voltages for test Board 1. The 16 iterations of the phase alignment algorithm adjust the varactor control voltage between $0 \mathrm{~V}$ and $2.5 \mathrm{~V}$. On iteration 17 varactor control voltage VCB overflows (Figure 6.13) and the algorithm is initialized on iteration 18. As was described in Section 5.4, we can see that the system did not converge. This is because the phase detector output, point B of Figure 5.11, is measuring a nominal $135^{\circ}$ instead of $45^{\circ}$, refer to the XOR transfer function of Figure 4.2. Additionally, 
the corresponding varactor control will need to correct in the opposite direction from the other varactor controls.

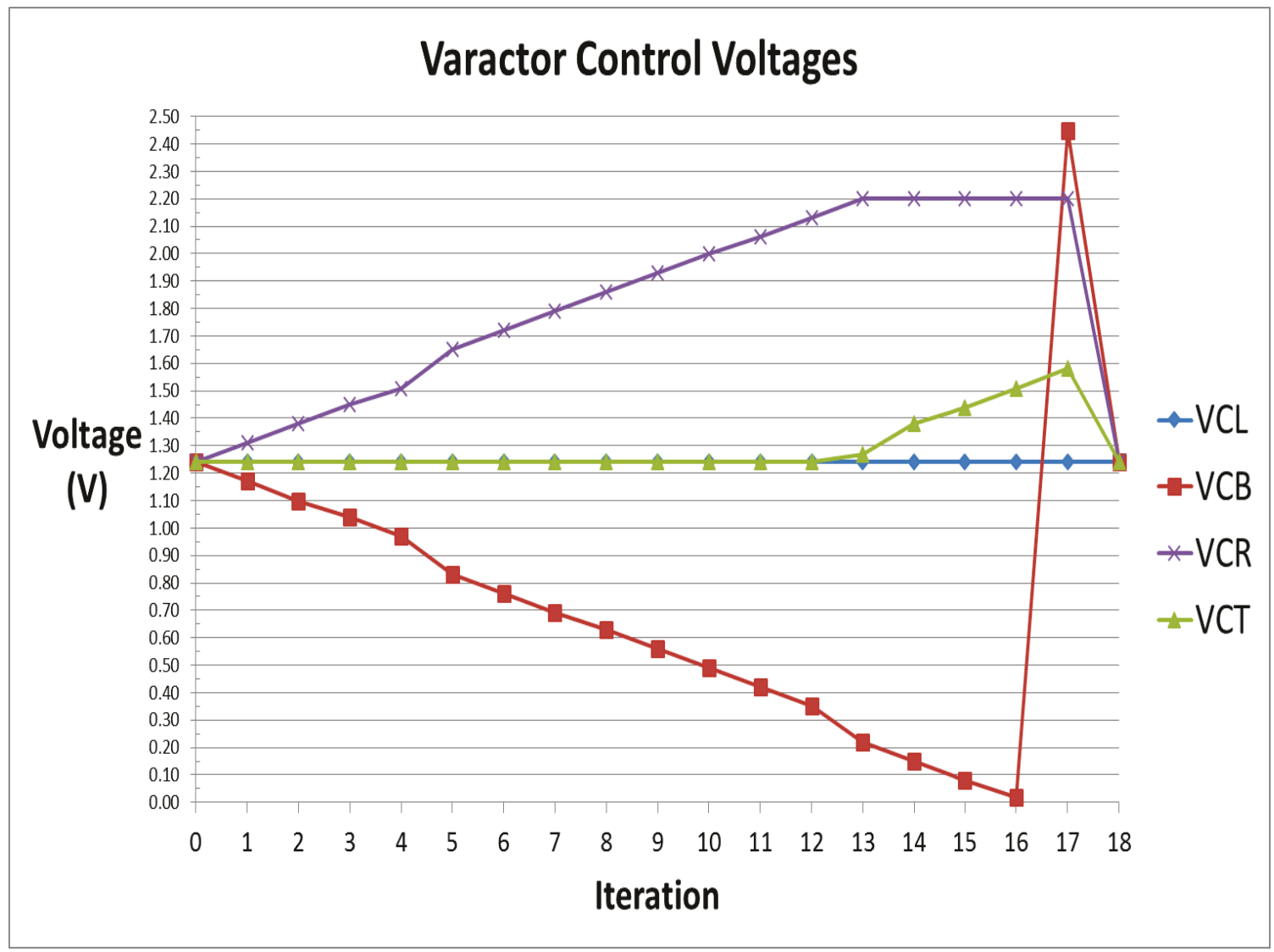

Figure 6.13: Test Board 1 Phase Detector Output Signals

Figure 6.14 shows the single-ended SXOR phase detector output voltages for test Board 2. These signals clearly diverge in a manner that is different than Board 1 . This may be due to manufacturing variation or direction of RTWO rotation. 


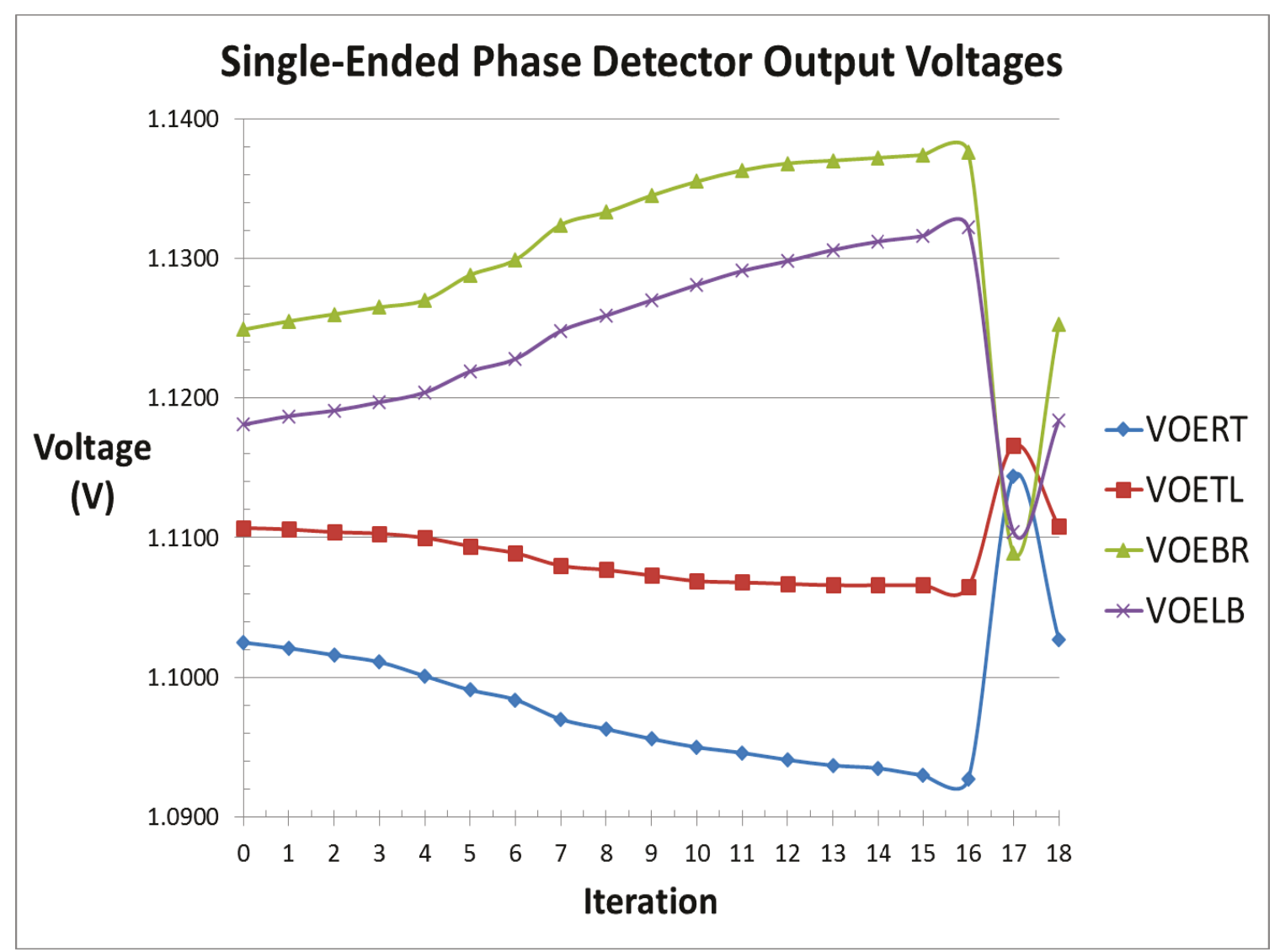

Figure 6.14: Test Board 2 Phase Detector Output Signals

Figure 6.15 shows the varactor control voltages that are attempting to correct the diverging phases of Figure 6.14. Here we see that the VCT and VCB control voltages run out of varactor range in 16 iterations and overflow on iteration 17 . The system is initialized at iteration 18. 


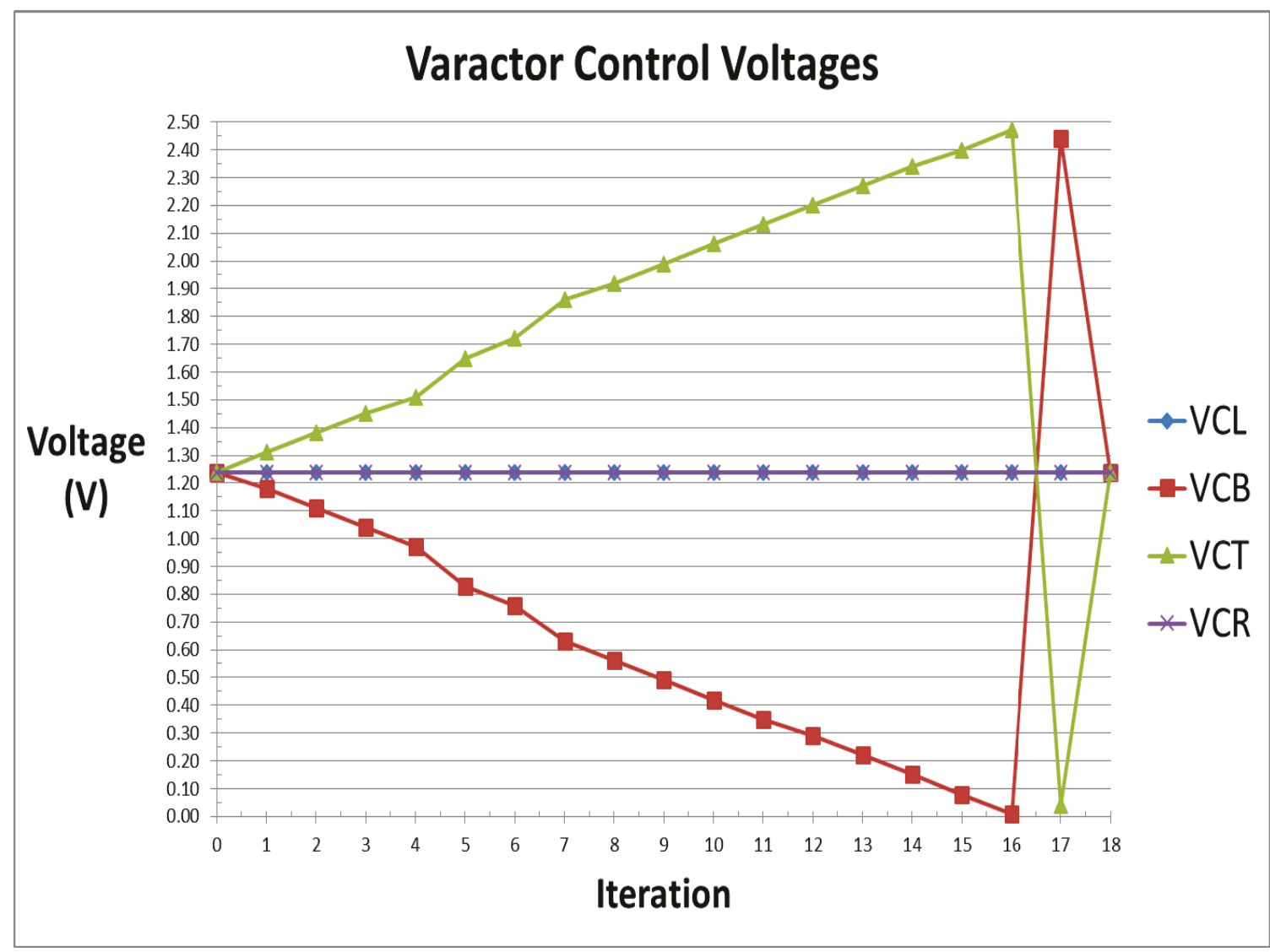

Figure 6.15: Test Board 2 Phase Detector Output Signals

During the testing of Boards 1 and 2 the RTWO frequency of oscillation was monitored. The frequency variation over the course of tuning is $\approx 150 \mathrm{MHz}$. This was expected and is similar to simulation results. This is clearly less than the frequency tuning range of $\approx 1.1 \mathrm{GHz}$.

The phase alignment algorithm will minimize the change in RTWO average frequency during phase tuning. That is, at each algorithm iteration a tuning voltage increase is made to one TL section and the tuning voltage is decreased by this same amount in another TL section. The objective here is to add and remove equal amounts of capacitance to maintain a constant average frequency of oscillation. However, the slope of the varactor capacitance curve changes with control voltage (see Figure 5.8); therefore, the total capacitance of the RTWO TL sections will change with phase 
tuning and the oscillating frequency will drift. Also, the frequency must be measured at a specific point on the RTWO and this frequency will change during phase tuning.

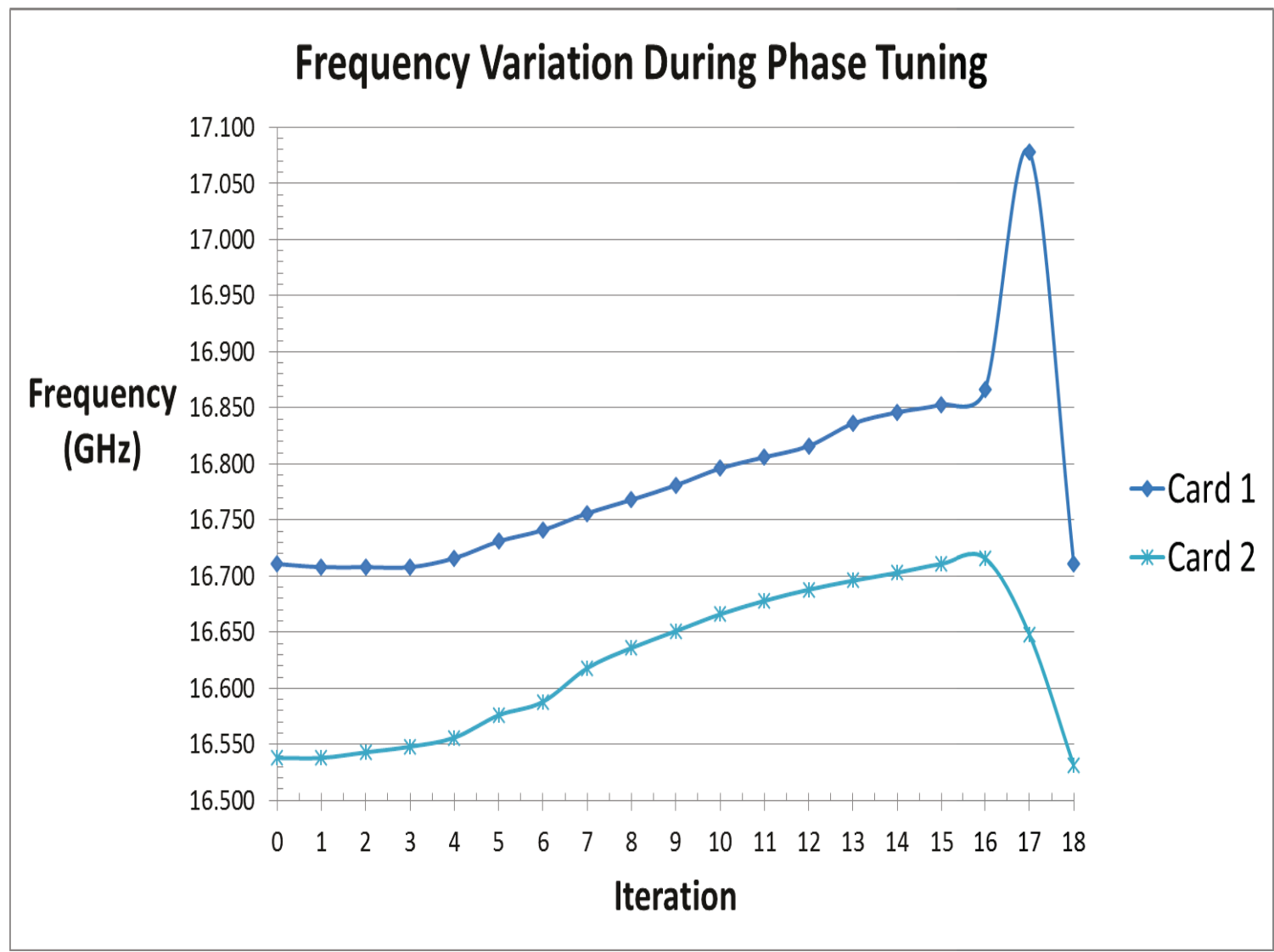

Figure 6.16: Test Board 2 Phase Detector Output Signals

\subsection{Discussion of Results - RTWO Phase Align- ment}

The SXOR phase detectors were used to measure and calibrate the phase of $45^{\circ}$ transmission line sections of an 18-GHz RTWO fabricated in SiGe BiCMOS. Although the convergence of the phase tuning was unsuccessful, the phase alignment algorithm was able to change the phase of the RTWO TL sections. The following was also demonstrated: 
1. The phase alignment algorithm implemented in verilog functioned correctly as can be seen in Figures 6.13 and 6.15 .

2. The phase alignment algorithm will minimize the change in RTWO average frequency during phase tuning. This change was $\approx 150 \mathrm{MHz}$ for the devices tested.

3. The reason the fabricated RTWO could not be tuned is understood. That is, the phase detector output signals are not the correct signals for the existing phase alignment algorithm. The phases being measured are $\Delta 1+180^{\circ}, \Delta 2$, $\Delta 3$, and $(\Delta 1+\Delta 2+\Delta 3)$. The voltage levels and the tuning direction for these signals need to be corrected by a revised version of the existing phase alignment algorithm. This work is currently under way and because these simulations take an extended period of time, are continuing on past the deadline for this document.

4. Additional varactor components should be added to each TL section to extend the phase tuning range. The length of the RTWO TL sections would then need to be reduced to compensate for the increase in total nominal capacitance in each TL section. That is, to maintain the same operating frequency.

5. In order to minimize power consumption, this RTWO phase calibration should be carried out during initial system power-on and thereafter, periodically or after frequency changes.

The following errors were addressed during the testing of the fabricated RTWO phase alignment test die.

\section{Systematic Error}


1. No calibration was required for this test set-up.

\section{Random Error}

1. The switch state changes used to control the FPGA code were debounced in the verilog code.

\section{Drift Error}

1. Test equipment was turned on and allowed to warm up to an operating temperature for at least 45 minutes before each test run.

2. All test runs were carried out without pauses. The objective here was to minimize the effects of room temperature and other drift mechanisms within the test equipment. 


\section{Chapter 7}

\section{Conclusions and Recommendations}

\subsection{Summary of Work}

Simple Symmetrical XOR (SXOR) phase detectors were designed, fabricated in SiGe BiCMOS and tested to resolve $1^{\circ}$ of phase error over $45^{\circ} \pm 10^{\circ}$ and $90^{\circ} \pm 10^{\circ}$ up to $12 \mathrm{GHz}$. This $12 \mathrm{GHz}$ limit is the maximum operating frequency of the test

set-up (i.e., limited by the Narda 3753B Phase Shifter), not the maximum operating frequency of the DUT. The following was also demonstrated:

1. In simulation this extracted phase detector can resolve $1^{\circ}$ of phase error over $45^{\circ} \pm 10^{\circ}$ and $90^{\circ} \pm 10^{\circ}$ up to a frequency of $20.0 \mathrm{GHz}$.

2. The differential transit delay through an XOR circuit can be equalized by using a symmetrical XOR or SXOR architecture.

3. The simple ECL outputs of these phase detectors are very sensitive to Vcc variation in single-ended operation. This problem can be mitigated by using the SXOR outputs differentially.

4. If multiple differential SXOR phase detectors are used to make phase measurements, these measurement values can be compared and used to make accurate 
RTWO phase adjustment. That is, relative phase measurements can be made using SXOR gates without the need of complex calibration functions. This has been demonstrated in simulation and is certainly possible in a fabricated circuit, although it has not been fully demonstrated here.

These phase detectors were used to measure and calibrate the phase of $45^{\circ}$ transmission line sections of an existing 18-GHz RTWO fabricated in SiGe BiCMOS. Although the convergence of the phase tuning was unsuccessful, the phase alignment algorithm was able to change the phase of the RTWO TL sections. The following was also demonstrated:

1. A simple phase alignment algorithm was implemented to phase tune an RTWO. This was successfully demonstrated using both Matlab/Simulink and Cadence AMS Designer.

2. The phase alignment algorithm will minimize the change in RTWO average frequency during phase tuning. That is, at each algorithm iteration a tuning voltage increase is made to one TL section and the tuning voltage is decreased by this same amount in another TL section. The objective here is to add and remove equal amounts of capacitance to maintain a constant average frequency of oscillation. However, the slope of the varactor capacitance curve changes with control voltage (see Figure 5.8); therefore, the total capacitance of the RTWO TL sections will change with phase tuning and the oscillating frequency will drift. Also, the frequency must be measured at a specific point on the RTWO and this frequency will change during phase tuning.

3. The phase alignment algorithm described in this work will converge the TL section phases of an RTWO to be within an acceptable tolerance value regardless of the RTWO direction of rotation. 
4. The reason the fabricated RTWO could not be tuned is understood. That is, the phase detector output signals are not the correct signals for the existing phase alignment algorithm. Also, the tuning direction for this incorrect signal will need to be reversed.

5. Equation (2.18) was derived and used to estimate the change in phase of an RTWO TL section for a change in varactor capacitance in that RTWO section.

\subsection{Contributions}

Simple Symmetrical XOR (SXOR) phase detectors were designed, fabricated in SiGe BiCMOS and tested to resolve $1^{\circ}$ of phase error over $45^{\circ} \pm 10^{\circ}$ and $90^{\circ} \pm 10^{\circ}$ up to $12 \mathrm{GHz}$. These phase detectors were used to measure and calibrate the phase of $45^{\circ}$ transmission line sections of an existing 18-GHz RTWO fabricated in SiGe BiCMOS. This phase tuning was carried out by a simple phase alignment algorithm that can be implemented easily in a hardware definition language, in this case verilog. This RTWO phase tuning system was shown to function correctly in simulation. Although the fabricated version did not function correctly, the circuit error is understood and can be corrected.

\subsection{Improvement and Future Work}

1. The current RTWO die was layed out in great haste to make a tape out date. The following improvements could be made to this circuit.

(a) The phase detector connection problems identified previously (Section 5.4, Figures 5.11, 5.12 and 5.13) could be corrected with a die revision. 
(b) Additional capacitive tuning elements components should be added to each TL section to extend the phase tuning range. The length of the RTWO TL sections would then need to be reduced to compensate for the increase in total nominal capacitance in each TL section. That is, to maintain the same operating frequency.

(c) The layout should be assessed and steps taken to reduce signal coupling and improve signal isolation (i.e., reduce feed-through at $f o$ and $2 f o$ ).

(d) Both positive and negative SXOR outputs should be brought out to pads.

(e) Sense lines should be installed to accurately measure Vcc and Ibias voltage levels for individual devices.

2. Op Amp circuits should be added to the differential SXOR outputs to produce phase measurement signals that are immune to Vcc variation.

3. In order to minimize power consumption, this RTWO phase calibration should be carried out during initial system power-on and thereafter, periodically or after frequency changes.

4. Cycle-to-cycle phase detection at $18 \mathrm{GHz}$ dissipates significant power. Additional methods of phase detection that consume less power should be explored. 


\section{List of References}

[1] K. Takinami and R. Walsworth, "Phase Error Calibration Technique for Rotary Traveling Wave Oscillators," IEEE Journal of Solid-State Circuits, vol. 45, pp. 2433-2444, Nov. 2010.

[2] D. S. Kim, H. Song, T. Kim, S. Kim, and D. K. Jeong, "A 0.3 - 1.4 GHz AllDigital Fractional-N PLL With Adaptive Loop Gain Controller," IEEE Journal of Solid-State Circuits, vol. 45, pp. 2300-2311, Nov. 2010.

[3] J. Zhuang, K. Waheed, and R. B. Staszewski, "A Technique to Reduce Phase/Frequency Modulation Bandwidth in a Polar RF Transmitter," IEEE Transactions on Circuits and Systems I: Regular Papers, vol. 57, pp. 2196-2207, Aug. 2010.

[4] T. Hentschel, "A Simple IQ-Regeneration Technique for Six-Port Communication Receivers," First International Symposium on Control, Communications and Signal Processing, pp. 311-314, 2004.

[5] K. Takinami, R. Walsworth, S. Osman, and S. Beccue, "Phase-Noise Analysis in Rotary Traveling-Wave Oscillators Using Simple Physical Model," IEEE Transactions on Microwave Theory and Techniques, vol. 58, pp. 1465-1474, June 2010.

[6] K. T. Ansari, T. N. Ross, and C. Plett, "Ku-Band High Output Power Multiphase Rotary Travelling-Wave VCO in SiGe BiCMOS," pp. 1-4.

[7] C. Zhuo, H. Zhang, R. Samanta, J. Hu, and K. C. K. Chen, "Modeling, optimization and control of Rotary Traveling-Wave Oscillator," 2007 IEEE/ACM International Conference on Computer-Aided Design, pp. 476-480, 2007.

[8] NXP Semiconductor, "QUBiC4X Design Manual Rev 4.0.1," 2011. 
[9] J. W. M. Rogers, C. Plett, and F. Dai, "Phase Detectors," in Integrated Circuit Design for High Speed Frequency Synthesis, ch. 2, pp. 46-52, Norwood, MA: Artech House, Inc., 2006.

[10] L. Schmidt and H. M. Rein, "New high-speed bipolar XOR gate with absolutely symmetrical circuit configuration," Electronics Letters, vol. 26, pp. 430-431, Mar. 1990.

[11] W. FANG, "Accurate Analytical Delay Expressions for ECL and CML Circuits and Their Applications to Optimizing High-speed Bipolar Circuits," IEEE Journal of Solid-State Circuits, vol. 25, pp. 572-583, Apr. 1990.

[12] G. Le Grand de Mercey, "A 18GHz rotary traveling wave VCO in CMOS with I/Q outputs," ESSCIRC 2004 - 29th European Solid-State Circuits Conference (IEEE Cat. No.03EX705), pp. 489-492.

[13] D. H. Oh, K. J. Choo, and D. K. Jeong, "Phase frequency detecting time-todigital converter," IEEE Electronic Letters, vol. 45, pp. 3-4, Feb. 2009.

[14] M. E. Heidari, M. Lee, and A. A. Abidi, "All-Digital Outphasing Modulator for a Software-Defined Transmitter," IEEE Journal of Solid-State Circuits, vol. 44, pp. 1260-1271, Apr. 2009.

[15] J. Wood, T. C. Edwards, S. Lipa, and S. Member, "Rotary Traveling-Wave Oscillator Arrays : A New Clock Technology," IEEE Journal of Solid-State Circuits, vol. 36, pp. 1654-1665, Nov. 2001.

[16] K. Takinami, R. Strandberg, P. C. P. Liagn, G. Le Grand De Mercey, T. Wong, and M. Hassibi, "A Rotary-Traveling-Wave-Oscillator-Based All-Digital PLL with a 32-Phase Embedded Phase-to-Digital Converter in 65nm CMOS," IEEE International Solid-State Circuits Conference, pp. 344-345, Feb. 2011.

[17] L. Rodoni, G. Von Büren, A. Huber, M. Schmatz, and H. Jäckel, "A 5.75 to 44 Gb/s Quarter Rate CDR With Data Rate Selection in 90 nm Bulk CMOS," IEEE Journal of Solid-State Circuits, vol. 44, pp. 1927-1941, July 2009.

[18] J. W. M. Rogers and C. Plett, "Image Rejection with Amplitude and Phase Mismatch," in Radio Frequency Integrated Circuit Design, ch. 8, pp. 274-276, Norwood, MA: Artech House, Inc., sceond ed., 2010. 
[19] G. Le Grand de Mercey, 18 GHz - 36 GHz Rotary Traveling Wave Voltage Controlled Oscillator in a CMOS Technology. PhD thesis, Universitat der Bundeswehr Munchen, Aug. 2004.

[20] B. S. Virdee, A. S. Virdee, and B. Y. Banyamin, Broadband Microwave Amplifiers. Norwood: Artech House, Inc., 2004.

[21] J. Beyer, S. Prasad, R. Becker, J. Nordman, and G. Hohenwarter, "MESFET Distributed Amplifier Design Guidelines," IEEE Transactions on Microwave Theory and Techniques, vol. 32, pp. 268-275, Mar. 1984.

[22] W. Andress and D. Ham, "Recent developments in standing-wave oscillator design: review." 2004.

[23] A. Mirzaei, M. Heidari, R. Bagheri, S. Chehrazi, and A. Abidi, "The Quadrature LC Oscillator: A Complete Portrait Based on Injection Locking," IEEE Journal of Solid-State Circuits, vol. 42, pp. 1916-1932, Sept. 2007.

[24] N. Nouri and J. F. Buckwalter, "A 45-GHz Rotary-Wave Voltage-Controlled Oscillator," IEEE Transactions on Microwave Theory and Techniques, vol. 59, pp. 383-392, Feb. 2011.

[25] Y. Chen and K. D. Pedrotti, "Rotary Traveling-Wave Oscillators, Analysis and Simulation," IEEE Transactions on Circuits and Systems, vol. 58, pp. 77-87, Jan. 2011.

[26] D. M. Pozar, "Equivalent Circuits for Two-Port Networks," in Microwave Engineering, ch. 4, p. 187, Amherst, MA: John Willey \& Sons, Inc., third ed., 2005.

[27] N. Haiyan and H. Jianping, "The Layout Implementations of High-Speed LowPower MCML Cells," International Conference on Electronics, Communciations and Control, pp. 2936-2939, 2011.

[28] G. Freeman, B. Jagannathan, S. J. Jeng, J. S. Rieh, A. D. Stricker, D. C. Ahlgren, and S. Subbanna, "Transistor Design and Application Considerations for," IEEE Transactions on Electron Devices, vol. 50, pp. 645-655, Mar. 2003.

[29] S. P. Voinigescu, T. O. Dickson, R. Beerkens, I. Khalid, and P. Westergaard, "A Comparison of Si CMOS, SiGe BiCMOS, and InP HBT Technologies for High-Speed and Millimeter-Wave ICs," IEEE 2004 Topical Meeting on Silicon 
Monolithic Integrated Circuits in RF Systems. Digest of Papers., pp. 111-114, 2004.

[30] NXP Semiconductor, "QUBiC4Xi Design manual Rev 1.0," 2008.

[31] T. O. Dickson, R. Beerkens, and S. P. Voinigescu, "A 2 . 5-V 45-Gb / s Decision Circuit Using SiGe BiCMOS Logic," IEEE Journal of Solid-State Circuits, vol. 40, pp. 994-1003, Apr. 2005.

[32] J. W. M. Rogers, C. Plett, and F. Dai, "Large-Signal Behavior of Bipolar and CMOS Differential Pairs," in Integrated Circuit Design for High Speed Frequency Synthesis, ch. 5, pp. 121-125, Norwood, MA: Artech House, Inc., first ed., 2006.

[33] J. W. M. Rogers and C. Plett, "Differential Pair (Emitter-Coupled Pair) and Other Differential Amplifiers," in Radio Frequency Integrated Circuit Design, ch. 6, pp. 183-184, Norwood, MA: Artech House, Inc., first ed., 2003.

[34] D. M. Pozar, "Formulas for Effective Dielectric Constant, Characteristic Impedance, and Attenuation," in Microwave Engineering, ch. 3, pp. 144-145, Amherst, MA: John Willey \& Sons, Inc., third ed., 2005.

[35] S. P. Voinigescu, "Static Dividers," in High-Frequency Integrated Cicrcuits, ch. 11, pp. 734-739, Cambridge, UK: Cambridge University Press, first ed., 2013.

[36] NXP Semiconductor, "QUBiC4 Platform Design Manual 4.06," 2011.

[37] NXP Semiconductor, "QUBiC4Xi Design Manual, Version 3.01," 2011.

[38] A. Hastings, "Matching of Resistors and Capacitors," in The Art of Analog Layout, ch. 7, pp. 214-259, Upper Saddle River, New Jersey: Prentice-Hall, Inc., first ed., 2001.

[39] T. Blalack, Y. Leclercq, and C. Yue, "On-chip RF isolation techniques," Proceedings of the Bipolar/BiCMOS Circuits and Technology Meeting, pp. 205-211, 2002.

[40] M. Pfost, P. Brenner, T. Huttner, and A. Romanyuk, "An experimental study on substrate coupling in bipolar/BiCMOS technologies," IEEE Journal of SolidState Circuits, vol. 39, pp. 1755-1763, Oct. 2004. 


\section{Appendix A}

\section{Additional Simulink RTWO Block Descriptions}

\section{A.1 Sub-Blocks of the RTWO $24 \mathrm{MHz}$ Block}

The gain stages of Figure 3.6 are illustrated in Figure A.1 at the next level of abstraction down. Here there are two summing nodes that add the input signals from the RTWO TL sections to their amplified complementary signals delayed by $180^{\circ}$, i.e., Integer Delay $Z^{-(F U S / 2)}$. The Amplifier blocks have enough gain to produce a $1.2 \mathrm{~V}$ square wave output from the attenuated input signal. The Saturation block prevents the amplified signal from exceeding $1.2 \mathrm{~V}$ as the power supply would in a CMOS inverter.

The sum of the incoming RTWO signal and its amplified complement will produce a signal that is larger than $1.2 \mathrm{~V}$ so it is limited by a Saturation block. The Transfer fcn blocks are LPFs that reduce the amplifier gain as frequency increases to mimic the gain bandwidth product of a real amplifier. Here alpha $=1 /(2 \pi F r e q \times N)$, where $\mathrm{N}$ is the number of harmonics to be included. It was found that $N \geq 20$ was required for the simulation to work well, and $N=60$ was used for the simulation results shown 
in this document. Rate Transition blocks are required by Simulink ${ }^{\circledR}$ where ever a signal path changes form continuous to discrete.

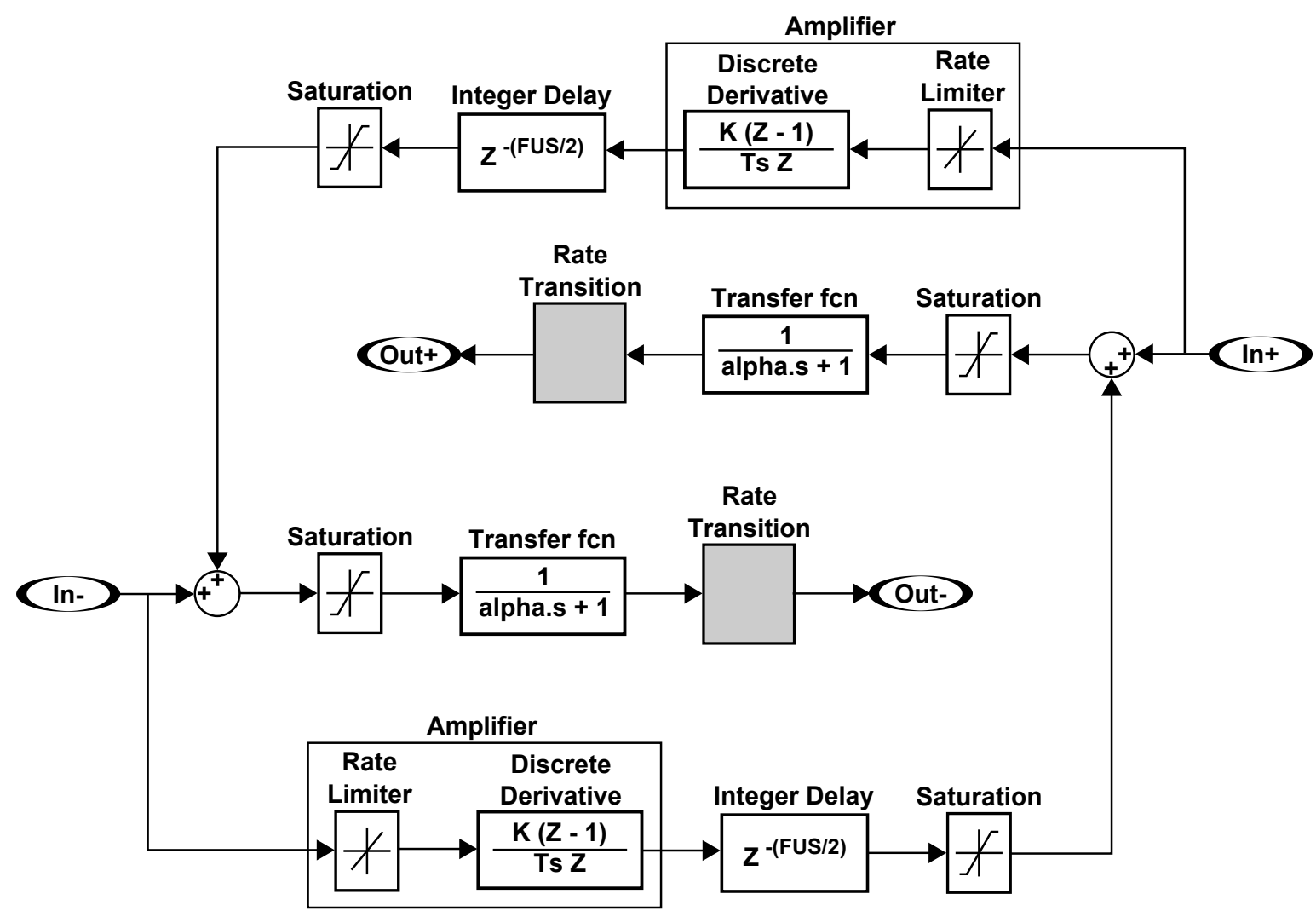

Figure A.1: RTWO Back-to-Back Gain Block

The Amplifier block is made of two sub-blocks, Rate Limiter and Discrete Derivative. The Rate Limiter block output is a positive slew rate signal when the input signal is high (i.e., $>0.6 \mathrm{~V}$ ) and a negative slew rate signal when the input signal is low or $<0.6 \mathrm{~V}$. The gain or slew rate for both positive and negative ramps is $24 \mathrm{MV} / \mathrm{s}$. The Discrete Derivative block differentiates these input positive and negative going ramps to produce a square wave output. The gain of this block is $5 \times 10^{-8}$, so the cascaded gains produce a square wave of amplitude $24 \times 10^{6} \times 5 \times 10^{-8}=1.2 \mathrm{~V}$. At this point, the question can be asked: why not implement this function with a simple amplifier followed by a Saturation block to limit the output signal level? There 
are two problems with this approach:

1. In a circuit simulator amplifier gain and output signal level are limited by the compression point of the amplifier (P1dB). The Simulink ${ }^{\circledR}$ amplifiers have no compression point; therefore, if there is more open-loop gain in the system than loss (a Barkhausen criterion), the RTWO travelling wave signal will accumulate gain and its level will head off to infinity as it rotates around the loop over and over again. The input signal can be mixed with itself, amplified and combined to create a third order component that will compression the gain. Unfortunately, this does not limit the output signal level and the travelling wave signal level heads off to infinity as before.

2. Simulink ${ }^{\circledR}$ amplifiers are simple multiply functions. That is, any signal level at the amplifier input is multiplied by the selected gain to produce an output signal. Therefore, if the low signal level is not exactly zero, but some very small positive value, it too will head to infinity as it continuously travels around the RTWO. In a circuit simulator the transistor based amplifiers drive the output signal to high and low levels that are limited by the power supply.

As long as the attenuation of any TL selection is less than $6.0 \mathrm{~dB}$ so the input high signal level of the Amplifier blocks of Figure A.1 is greater than $0.6 \mathrm{~V}$, the output signal will have a high level of $1.2 \mathrm{~V}$ and a low level of $0 \mathrm{~V}$.

The controllable delay blocks of Figure 3.6 (i.e., Delay 1 to Delay 4) are shown in Figure A.2. These Variable Integer Delay blocks are programmed to provide discrete delay from input to output. Here the output signal is the input signal delayed by i TSs. These blocks are initialized to 250 TS by the Phase Alignment Algorithm. 


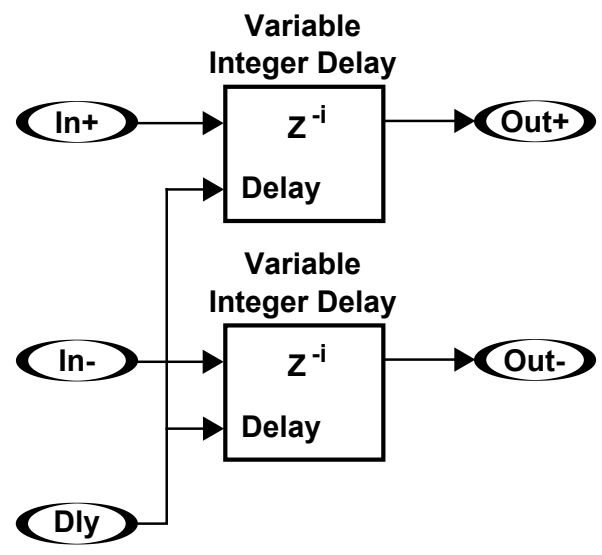

Figure A.2: RTWO Variable Integer Delay Block

Figure A.3 shows the details of the $45^{\circ}$ Section block of Figure 3.6. Here, from input to output, we have TL section Loss and LPF elements followed by a Rate Transition block to convert the continuous signal to a discrete signal so a nominal $45^{\circ}$ delay can be applied (i.e., FUS/8) with block Integer Delay. The offset_n portion of this variable is present in each TL section (i.e., offset_1 to offset_4) and is used to insert a initial system phase skew so the Phase Alignment Algorithm (Figure 3.9) will have something to correct. The setup.m file initializes Loss $=0.6$ and alpha $=1 /(2 \pi$ Freq $\times N)$, where $N=60$ is the number of harmonics to be included.

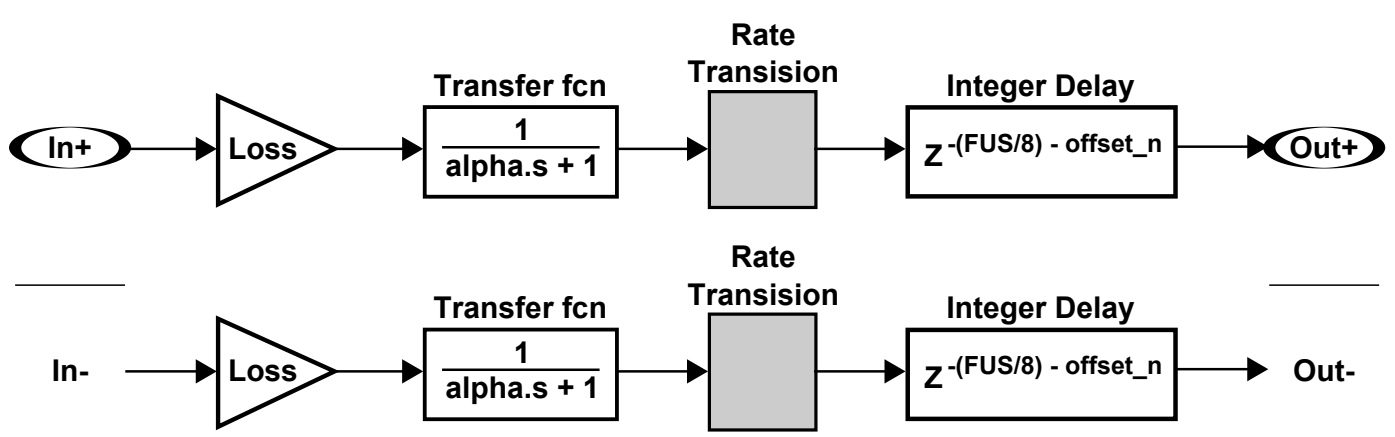

Figure A.3: RTWO 45 Degree Section 


\section{A.2 Gain Blocks}

The implementation of the CML buffers of Figure 3.1 is illustrated in Figure A.4. The differential input signals levels are halved and the negative input is subtracted from the positive input. The result is applied to two gain stages, \pm 0.5833 , to produce the CML amplitude swing of $350 \mathrm{mV}$. A $0.85 \mathrm{~V}$ bias is then applied to these signals to mimic a CML signal at the outputs. That is, $V_{O H}=1.2 \mathrm{~V}$ and $V_{O L}=0.5 \mathrm{~V}$.

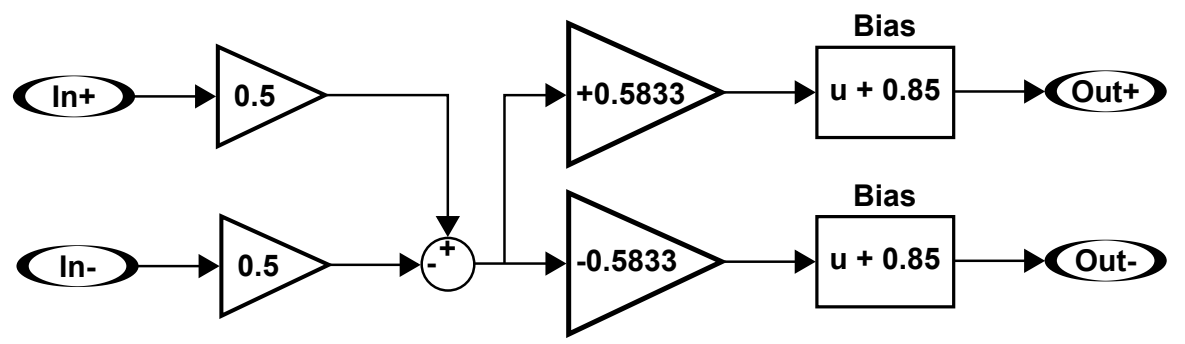

Figure A.4: CML Buffer for RTWO Phase Taps

The differential in to single-ended out buffer of Figure 3.1 is shown in Figure A.5. The negative differential CML input is subtracted from the positive differential CML input and the resulting amplitude is halved and biased at $0.85 \mathrm{~V}$ to produce a single-ended output, $V_{O H}=1.2 \mathrm{~V}$ and $V_{O L}=0.5 \mathrm{~V}$.

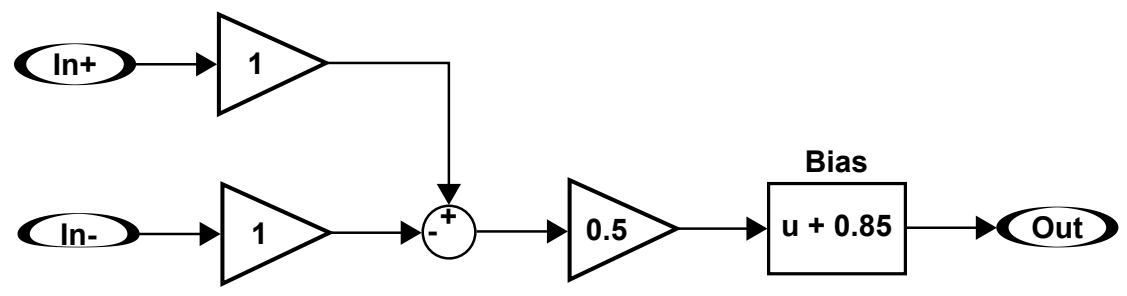

Figure A.5: Differential In to Single-Ended Out Buffer

In A.6 the single-ended buffer at the filtered output of the XOR phase detector of Figure 3.1 is described. The buffer biases the input voltage at $-0.6 \mathrm{~V}$ to remove its dc offset so the signal can be amplified by five without exceeding the $1.2 \mathrm{~V}$ input 
range of the ADC. This can be considered an effective coding gain of $14.0 \mathrm{~dB}$ for the ADC function, which will improve the tuning resolution of the system.

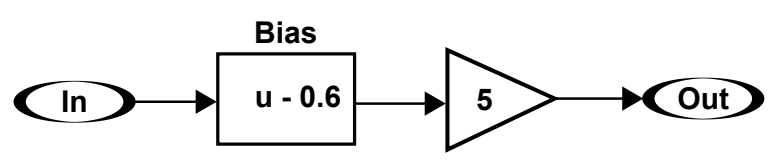

Figure A.6: Single-Ended Buffer 


\section{Appendix B}

\section{Phase Calibration Algorithm Code}

\section{B.1 Cadence Verilog Code}

The following Verilog code is resident in the Phase Alignment Algorithm block of Figure 3.10.

// Verilog HDL for 4 Section RTWO Phase Calibration.

module Phase_Cal_1(T1, T0, CCW, TCo,LCo, BCo, RCo, BPi , RPi , TPi , LPi , Status) ;

\begin{tabular}{|c|c|c|}
\hline input & \multicolumn{2}{|c|}{$\mathrm{T} 1, \mathrm{~T} 0, \mathrm{CCW}$} \\
\hline input & {$[11: 0]$} & $\mathrm{BPi}, \mathrm{RP} i, \mathrm{TP} i, \mathrm{LP} i$ \\
\hline output & {$[11: 0]$} & Status; \\
\hline output & {$[8: 0]$} & TCo , LCo , BCo , RCo; \\
\hline wire & \multicolumn{2}{|c|}{$\mathrm{T} 1, \mathrm{~T} 0, \mathrm{CCW}$} \\
\hline wire & {$[11: 0]$} & BPi , RPi , TPi , LPi ; \\
\hline wire & {$[11: 0]$} & Status; \\
\hline wire & {$[8: 0]$} & TCo , LCo , BCo , RCo ; \\
\hline reg & {$[11: 0]$} & MaxVal, MinVal, Limit, Outsig; \\
\hline reg & {$[8: 0]$} & sum1, sum2, sum3, sum 4 \\
\hline reg & {$[2: 0]$} & Maxi,Mini; \\
\hline reg & {$[3: 0]$} & Delta; \\
\hline
\end{tabular}




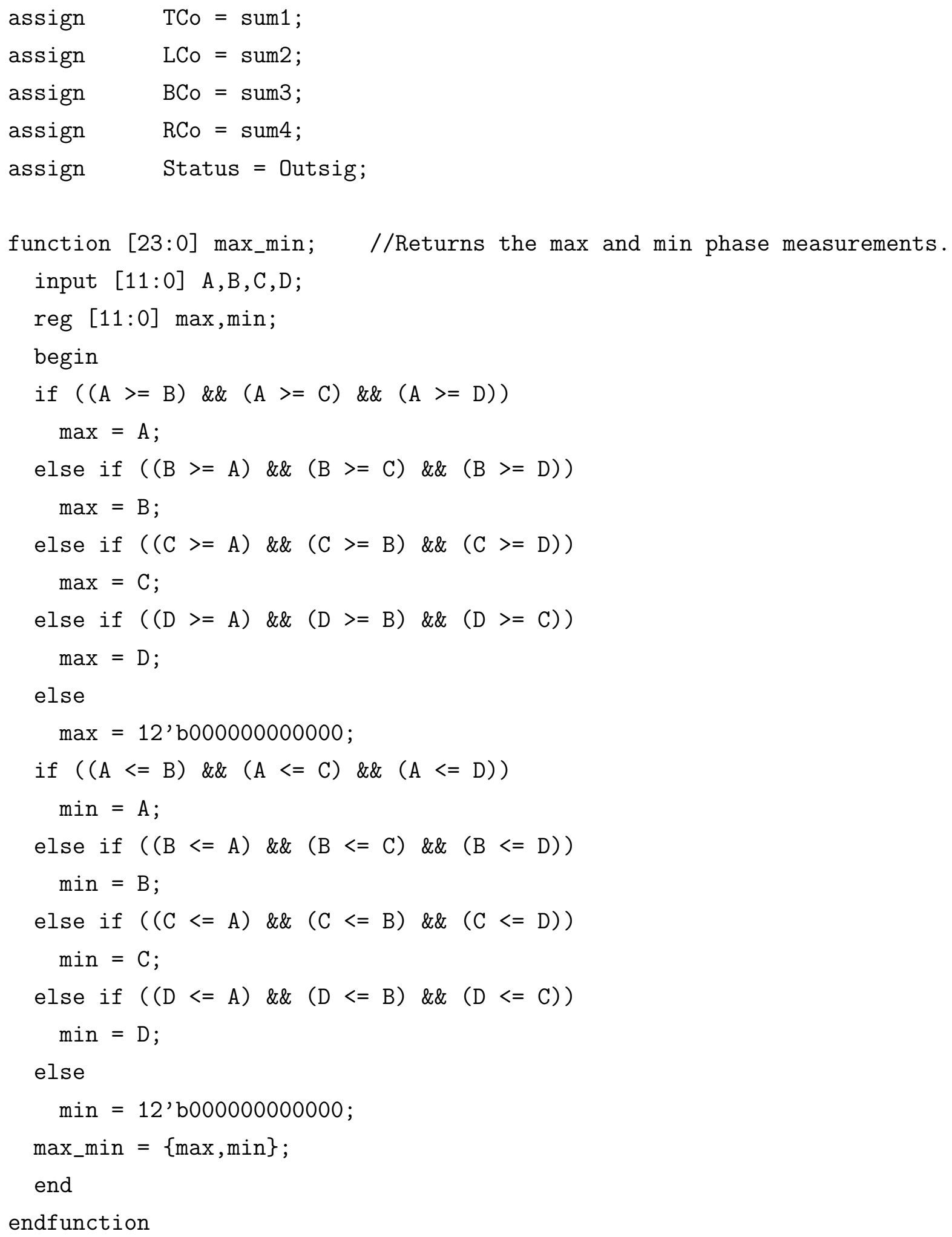




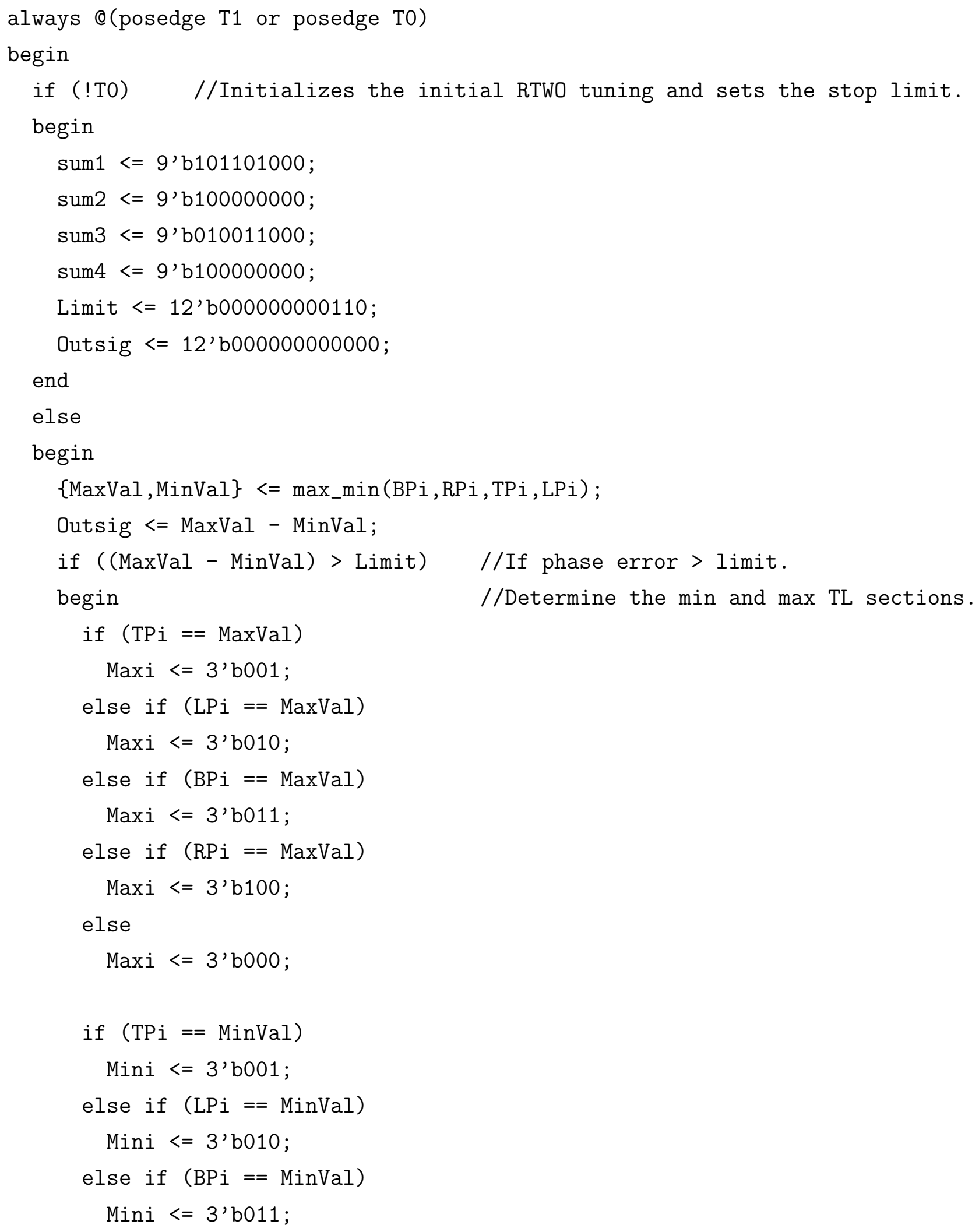




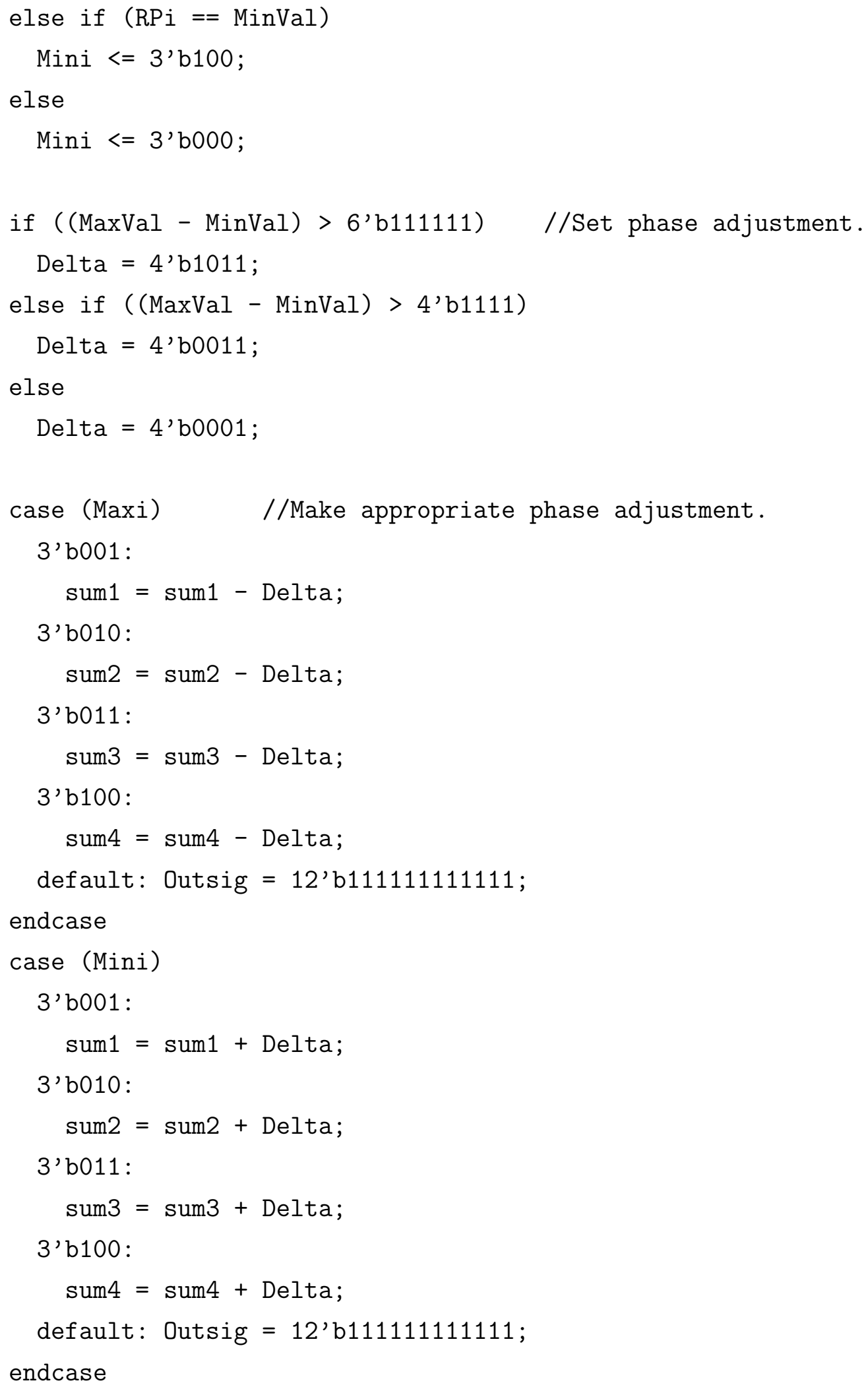




end
end
end
endmodule

\section{B.2 FPGA Verilog Code}

The following Verilog code is resident in the Phase Alignment Algorithm block of Figure 6.10. The main program is DAC_ADC_prog_2, which calls IBUFG to buffer the Spartan-6 FPGA oscillator input; debounce to qualify the initialization and iteration external switch inputs; and Phase_Cal_Mult_2 to calculate the new varactor voltages from the current phase detector output voltages.

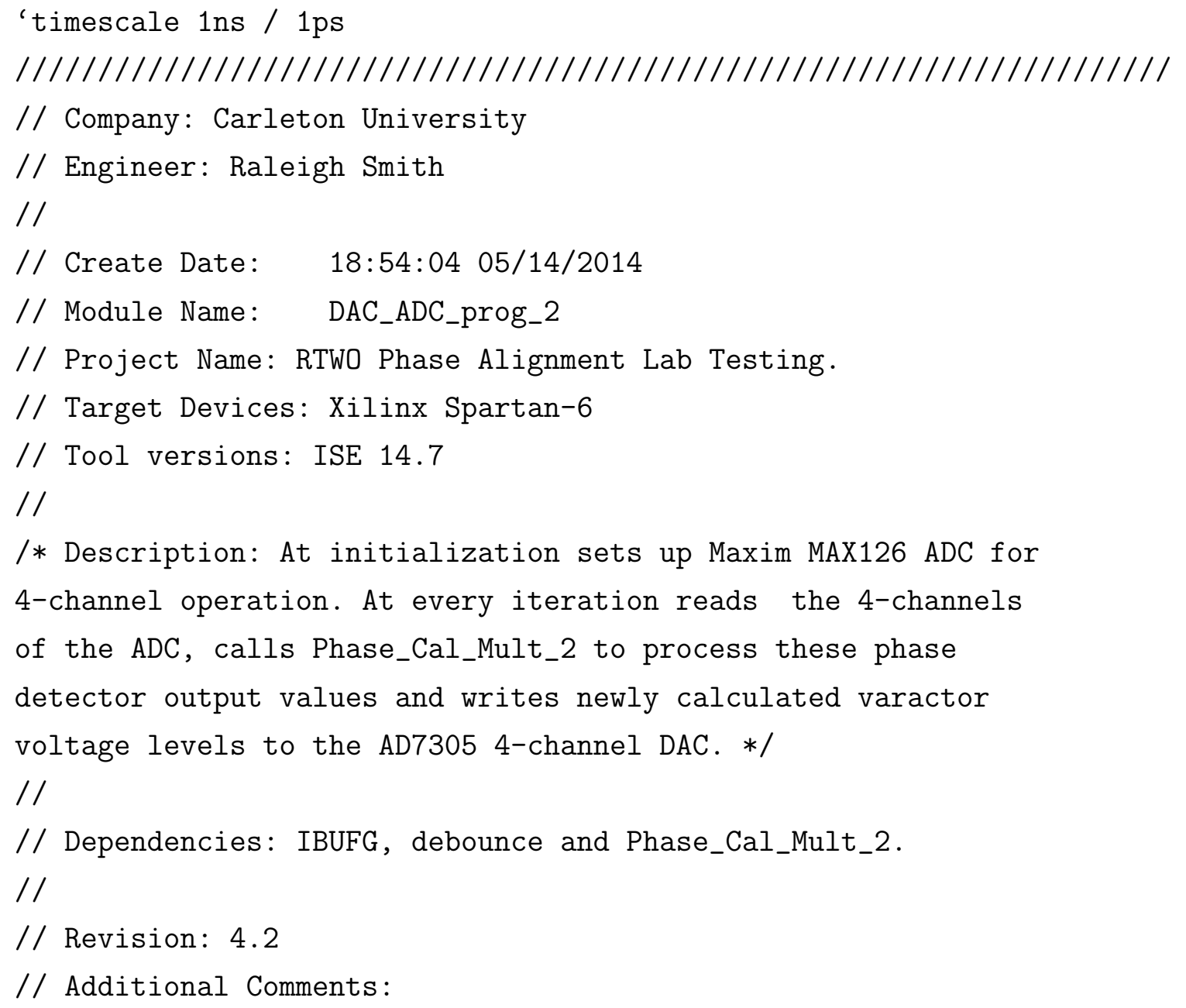


//

$1 / / / / / / / / / / / / / / / / / / / / / / / / / / / / / / / / / / / / / / / / / / / / / / / / / / / / / / / / / / / / / /$ module DAC_ADC_prog_2(CONVSTb, CSb, RDb, WRb, INTb, A, TO, T1, AD_WRb, Add, Data, LDACb, clock);

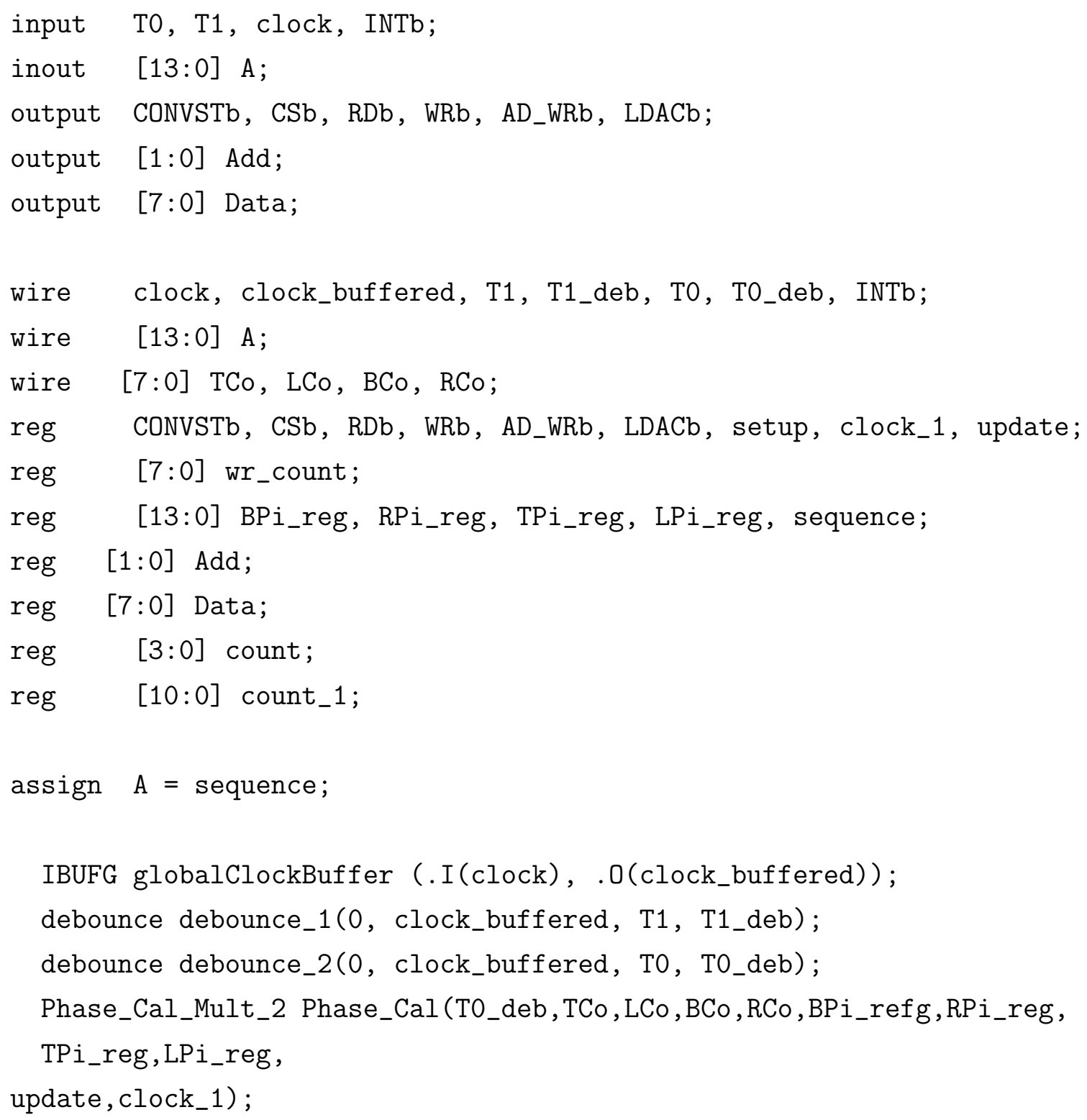




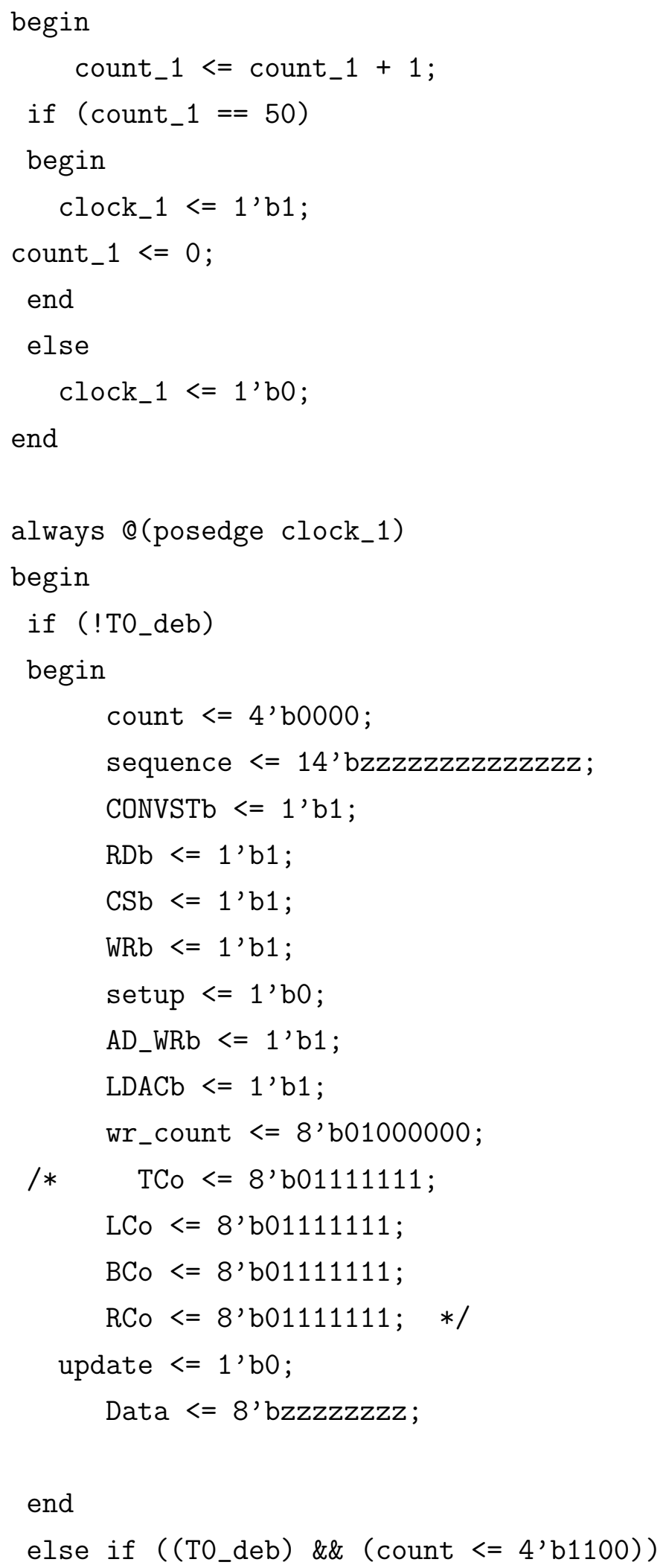




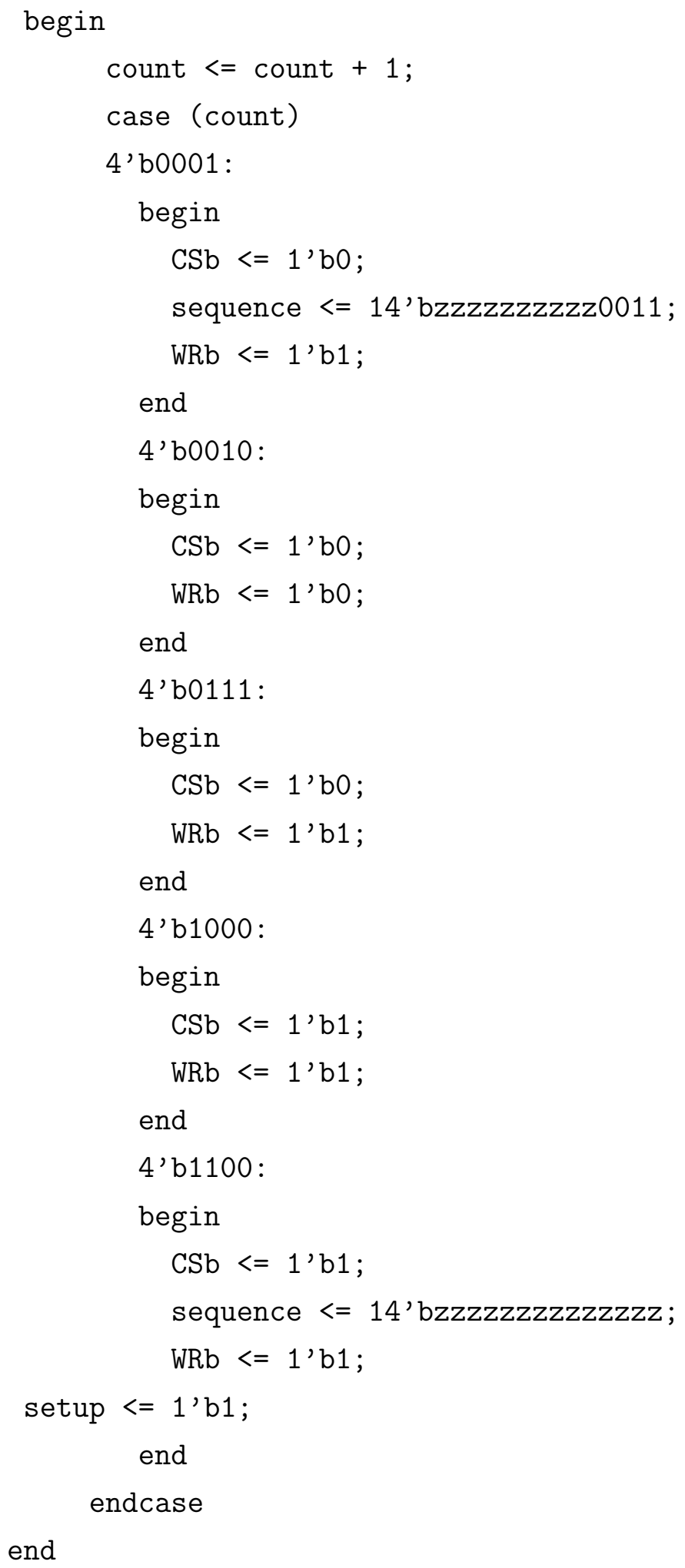




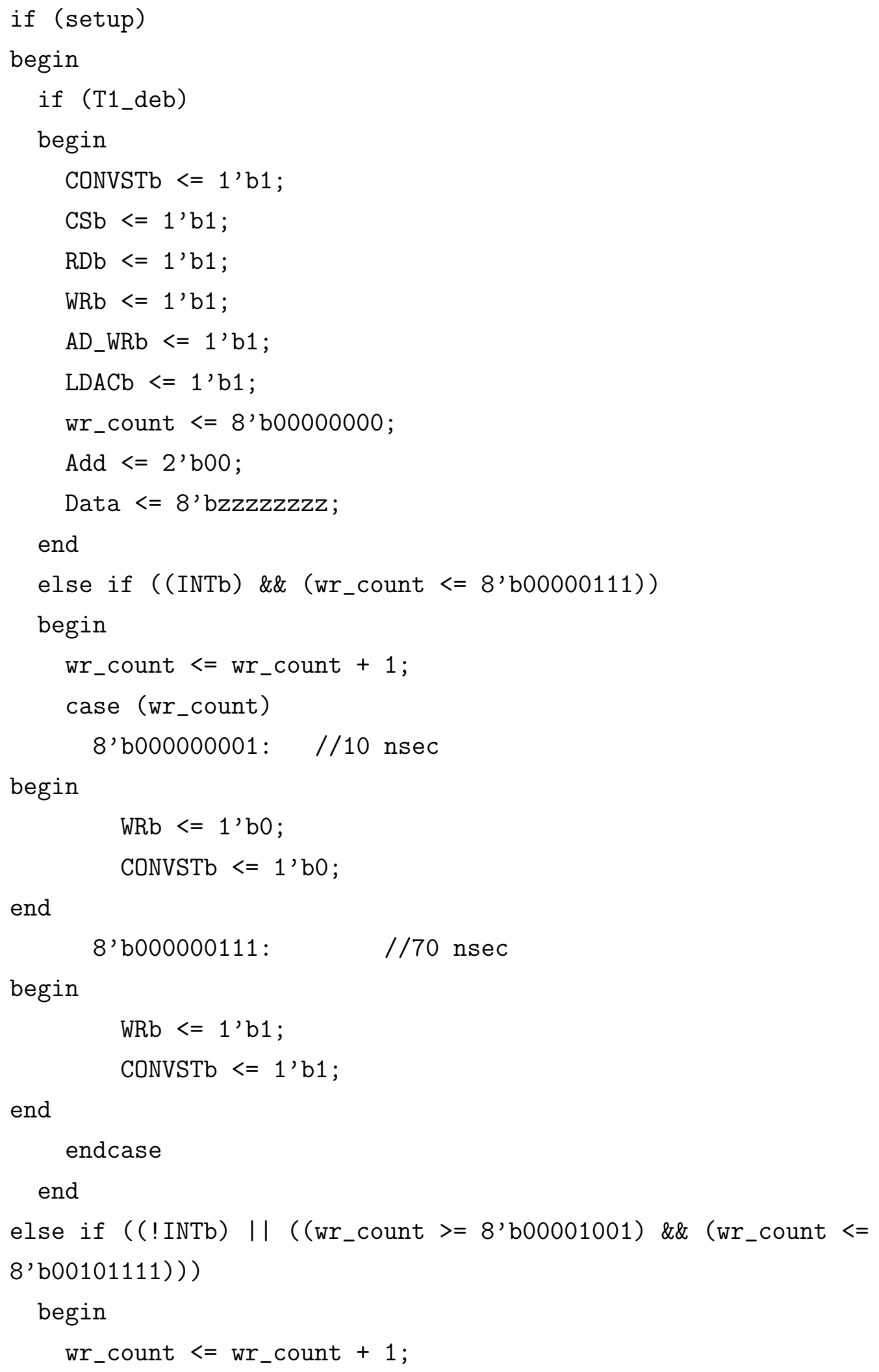




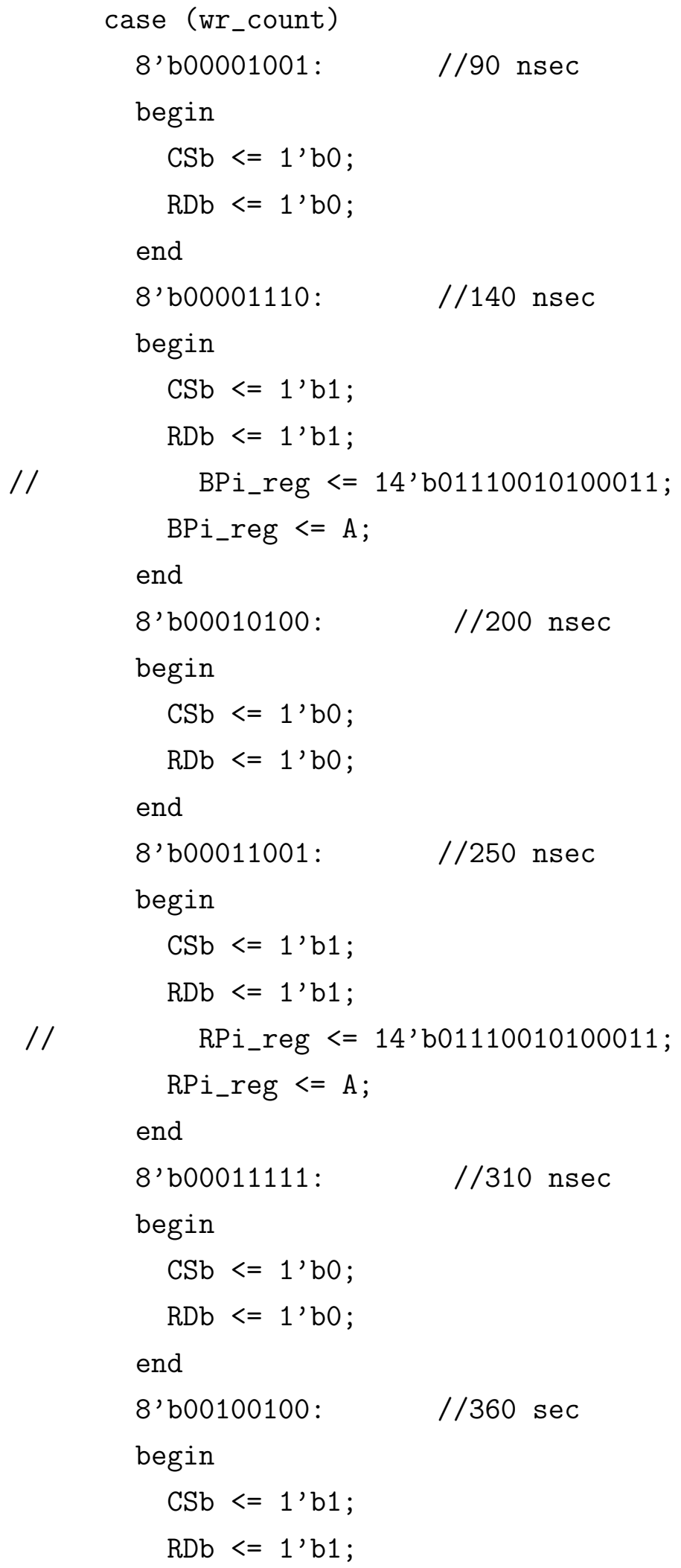




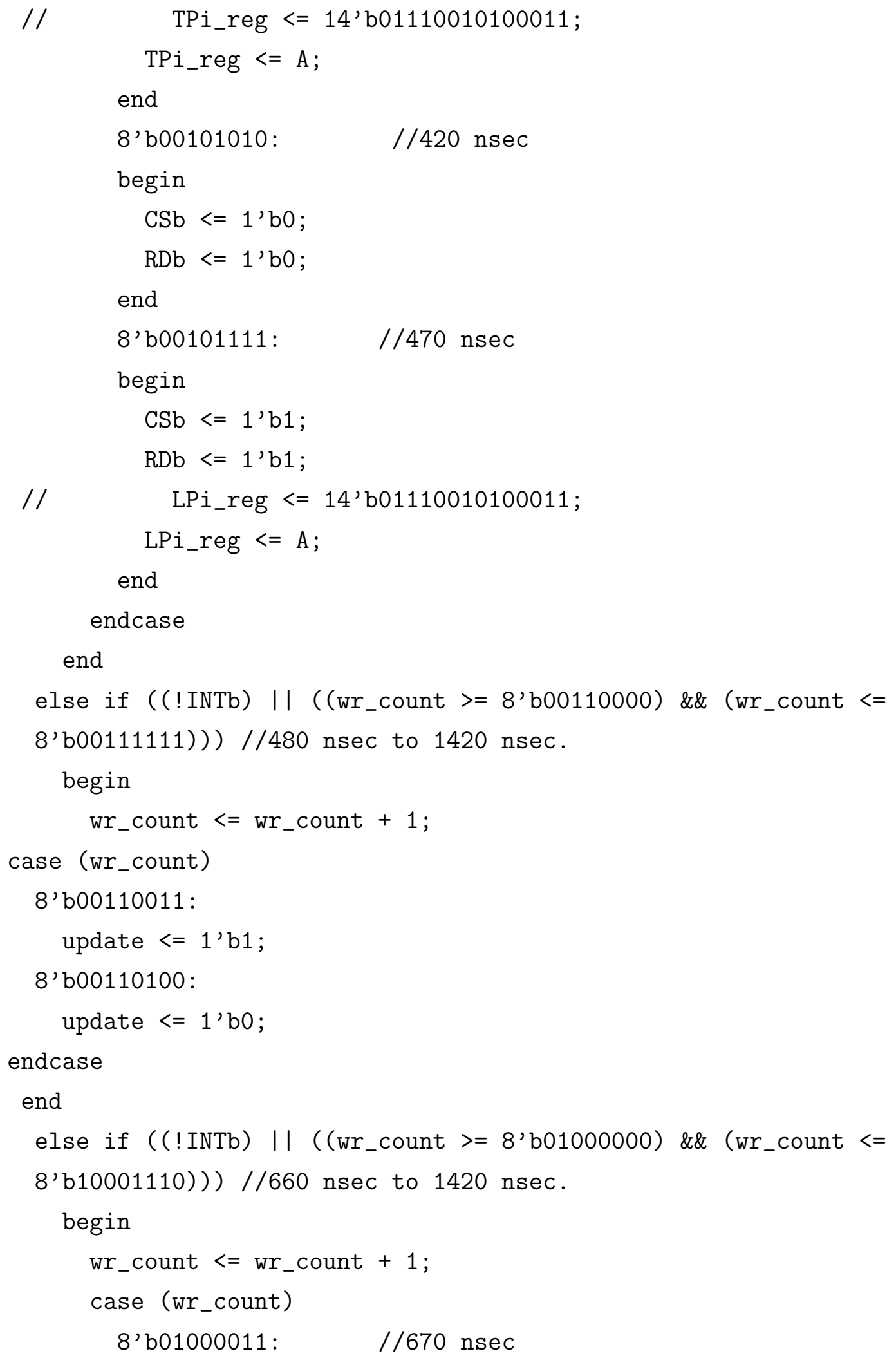




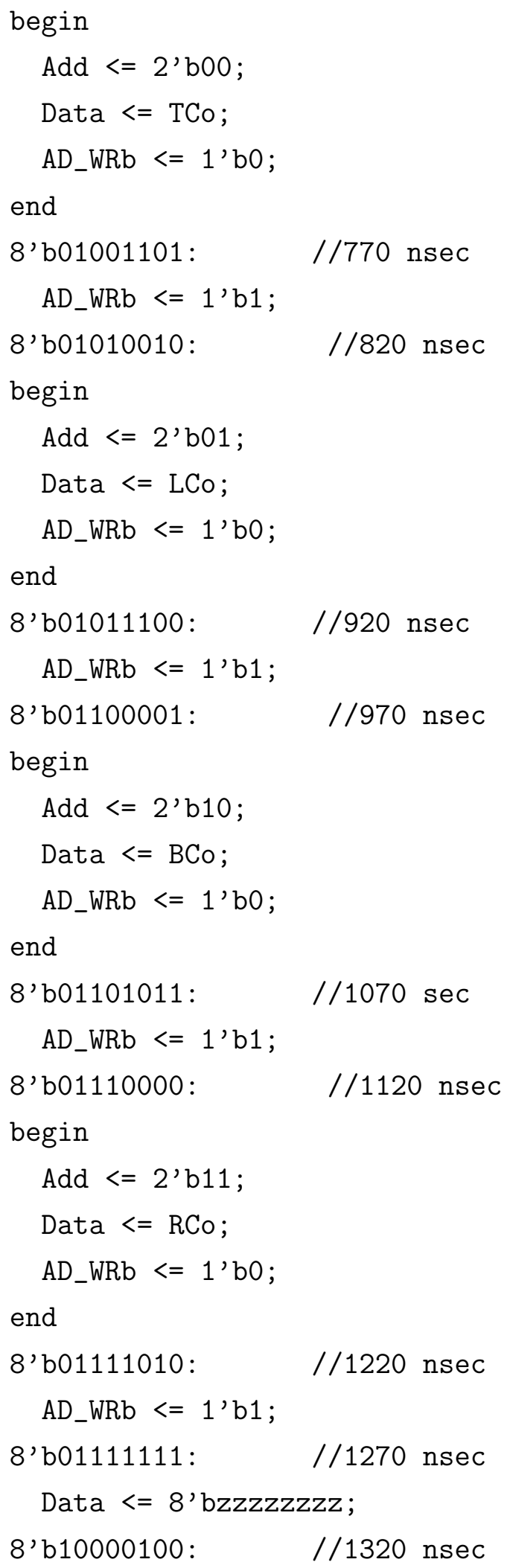




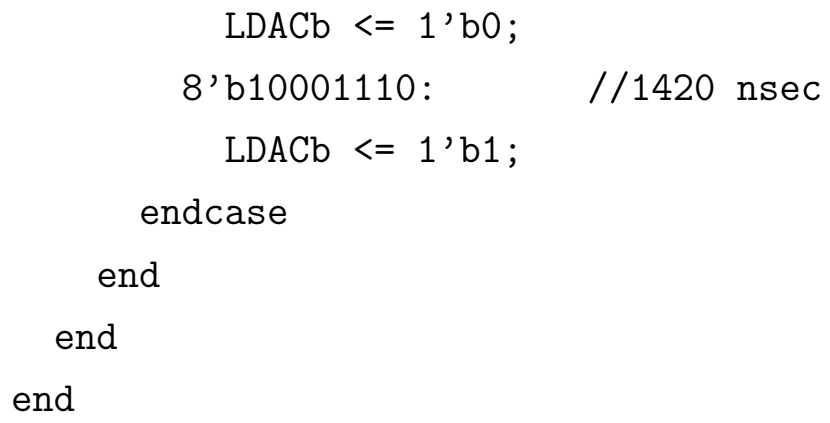




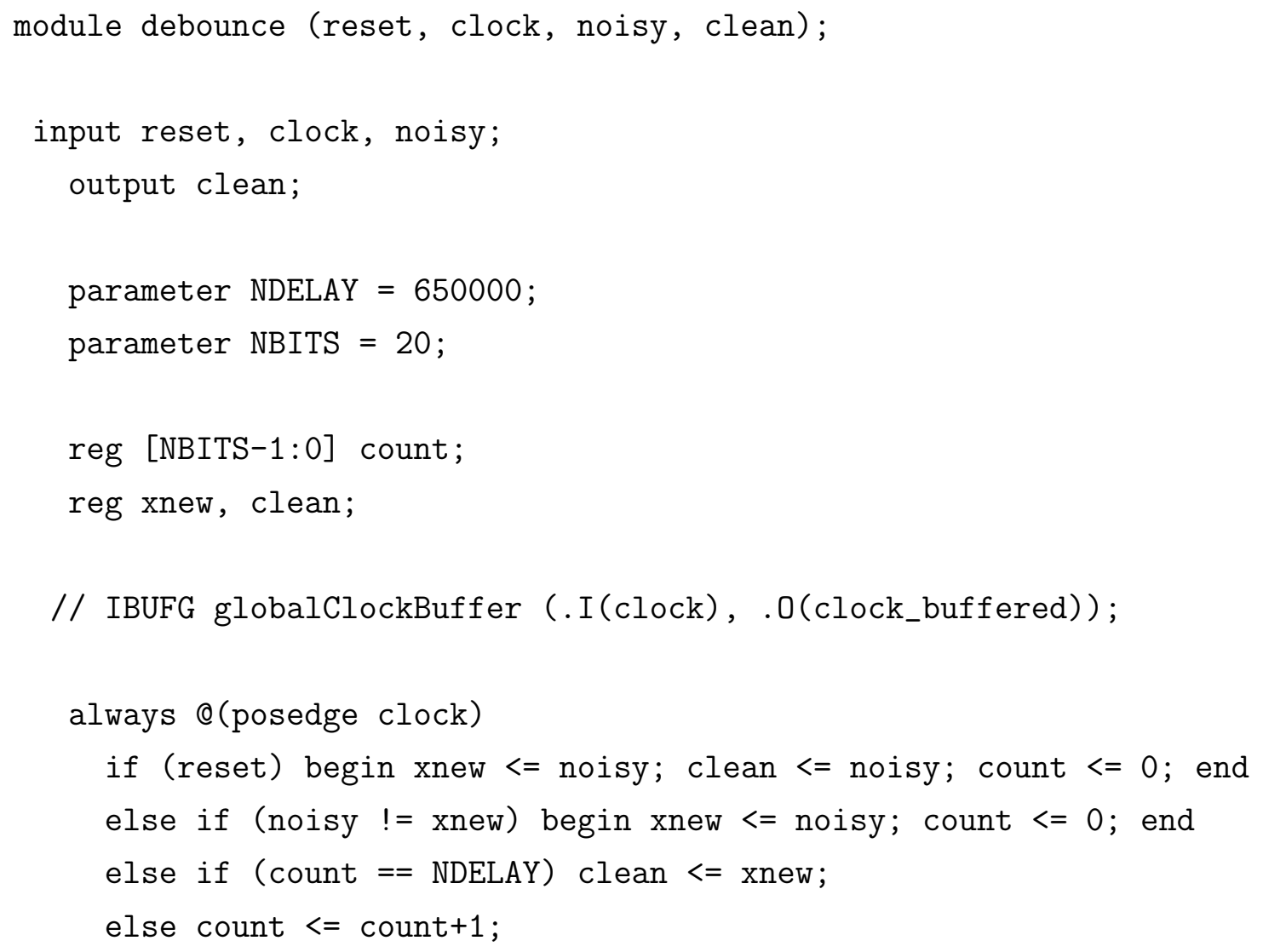

endmodule

'timescale 1ns / 1ps

///////////////////////////////////////////////////////////////

// Company: Carleton University

// Engineer: Raleigh Smith

$/ /$

// Create Date: 20:21:51 05/19/2014

// Module Name: Phase_Cal_Mult_2

// Project Name: RTWO Phase Alignment Lab Testing.

// Target Devices: Xilinx Spartan-6

// Tool versions: ISE 14.7

/* Description: This program creates new varactor voltage levels from the current phase detector voltage levels.*/ 
//

// Dependencies: A buffered clock and valid phase detector data.

//

// Revision: many.

// Additional Comments:

//

////////////////////////////////////////////////////////////

module Phase_Cal_Mult_2(T0,TCo,LCo,BCo,RCo,BPi, RPi , TPi , LPi , update, clock);

//inout update;

input To, update, clock;

input [13:0] BPi,RPi,TPi,LPi;

output $\quad[7: 0] \quad$ TCo, LCo, BCo, RCo;

wire T0, update, clock;

wire [13:0] BPi,RPi,TPi,LPi;

wire $\quad[7: 0] \quad$ TCo, LCo, BCo, RCo;

$\begin{array}{lll}\text { reg } & {[13: 0]} & \text { Limit; } \\ \text { reg } & {[7: 0] \quad \text { sum1, sum2, sum3, sum4; }} \\ \text { reg } & {[2: 0] \quad \text { Maxi,Mini, Delta; }} \\ \text { reg } & {[13: 0]} & \max , \min ;\end{array}$

assign $\quad$ TCo $=$ sum1;

assign $\quad$ LCo $=$ sum2;

assign $\quad \mathrm{BCo}=$ sum3;

assign $\quad$ RCo $=$ sum4;

always $@(B P i, R P i, T P i, L P i)$

begin

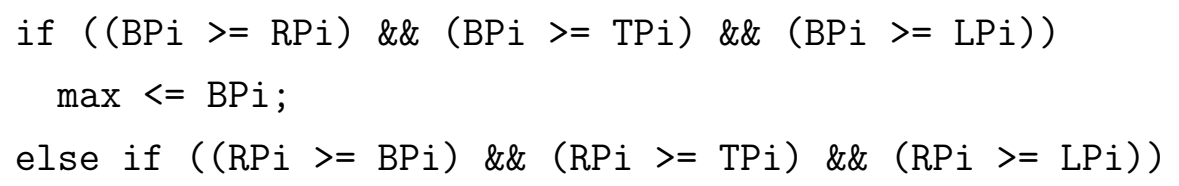




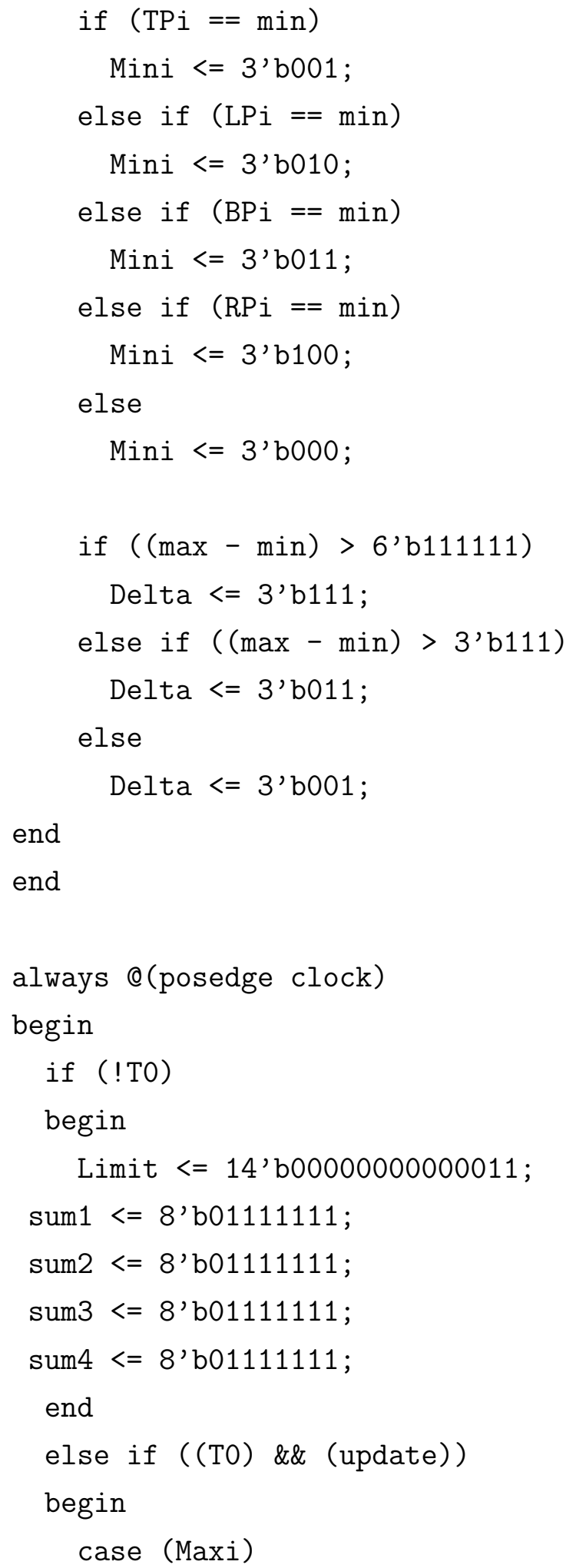




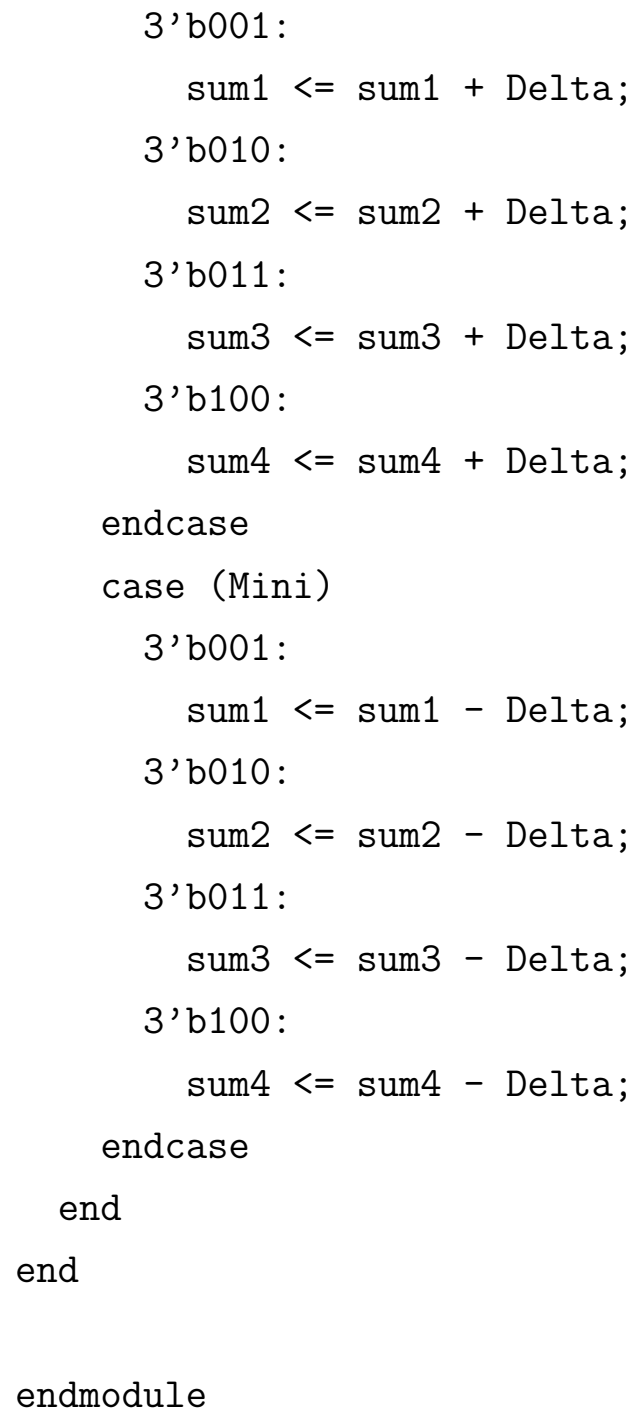

\section{B.3 Matlab Code}

The following is Matlab code is resident in the Phase Alignment Algorithm block of Figure 3.8.

function [RST, stp, No, TOTo1, TOTo2, TOTo3, TOTo4, Out1, Out2, Out3, Out4, Dly1,Dly2,Dly3,Dly4] = fcn (T0, T1, In1, In2, In3, In4, Ni, TOTi1, TOTi2, TOTi3, TOTi4, In1_, In2_, In3_, In4 , D1, D2, D3, D4) $\%$ This block supports an embeddable subset of the MATLAB language. 


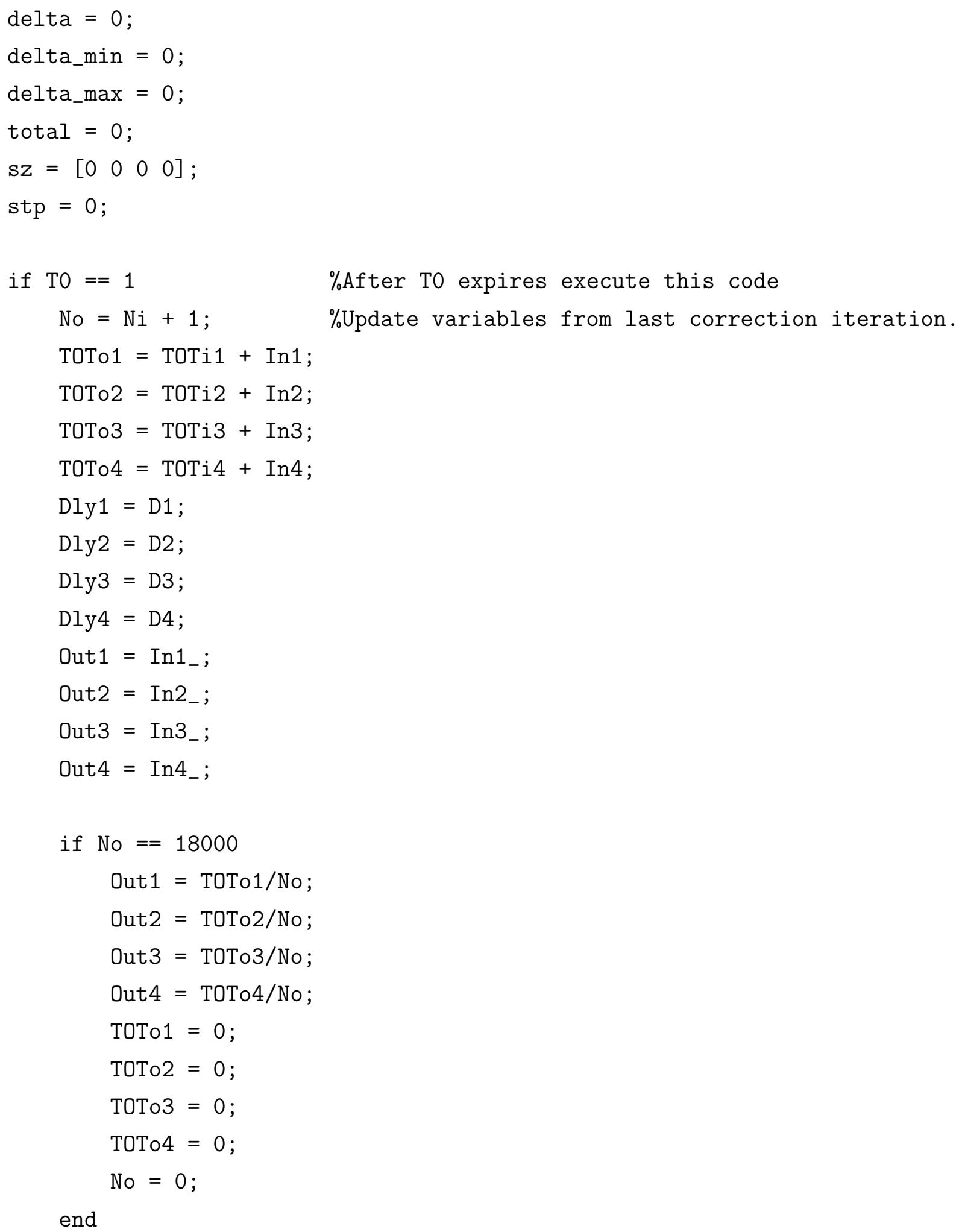




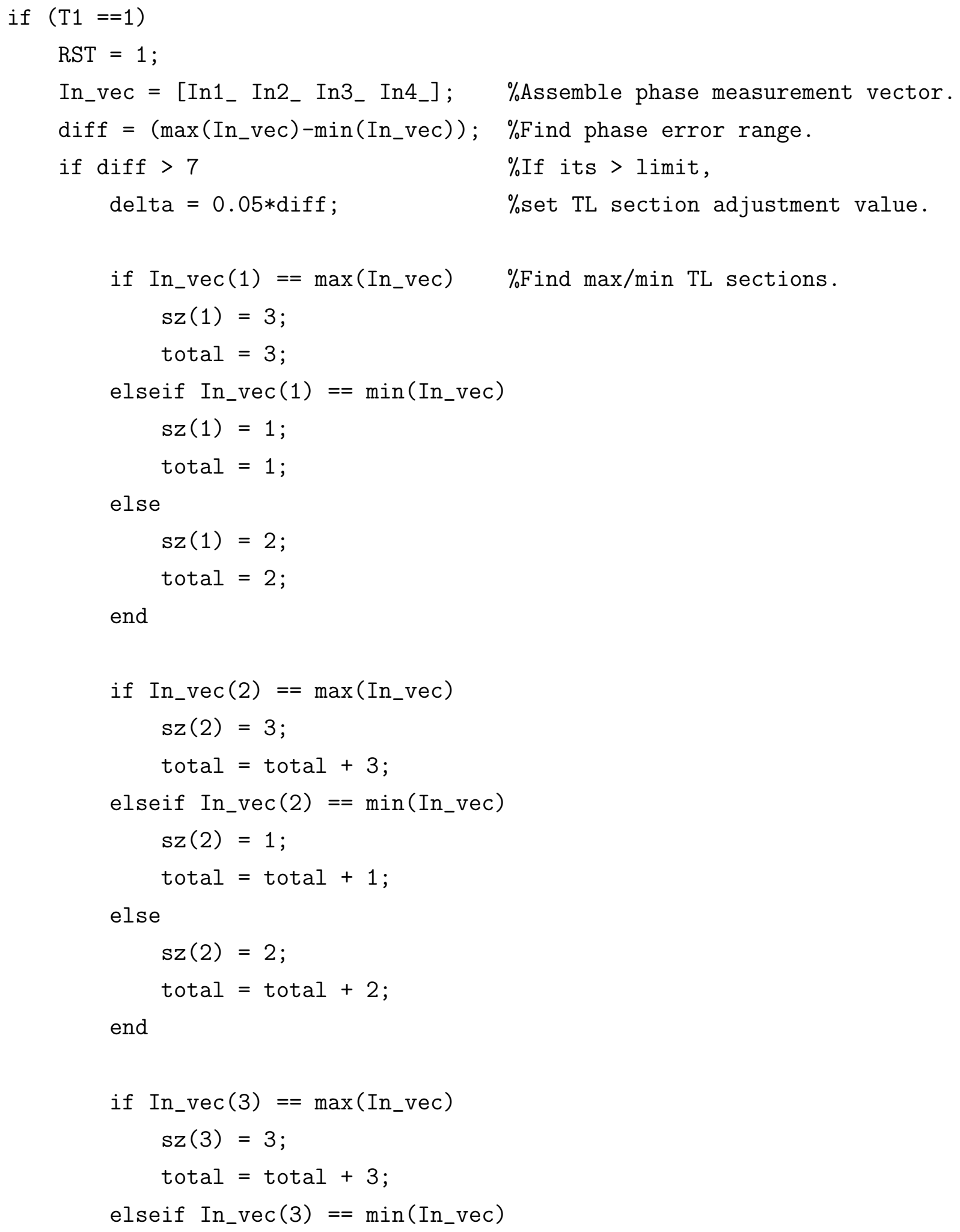




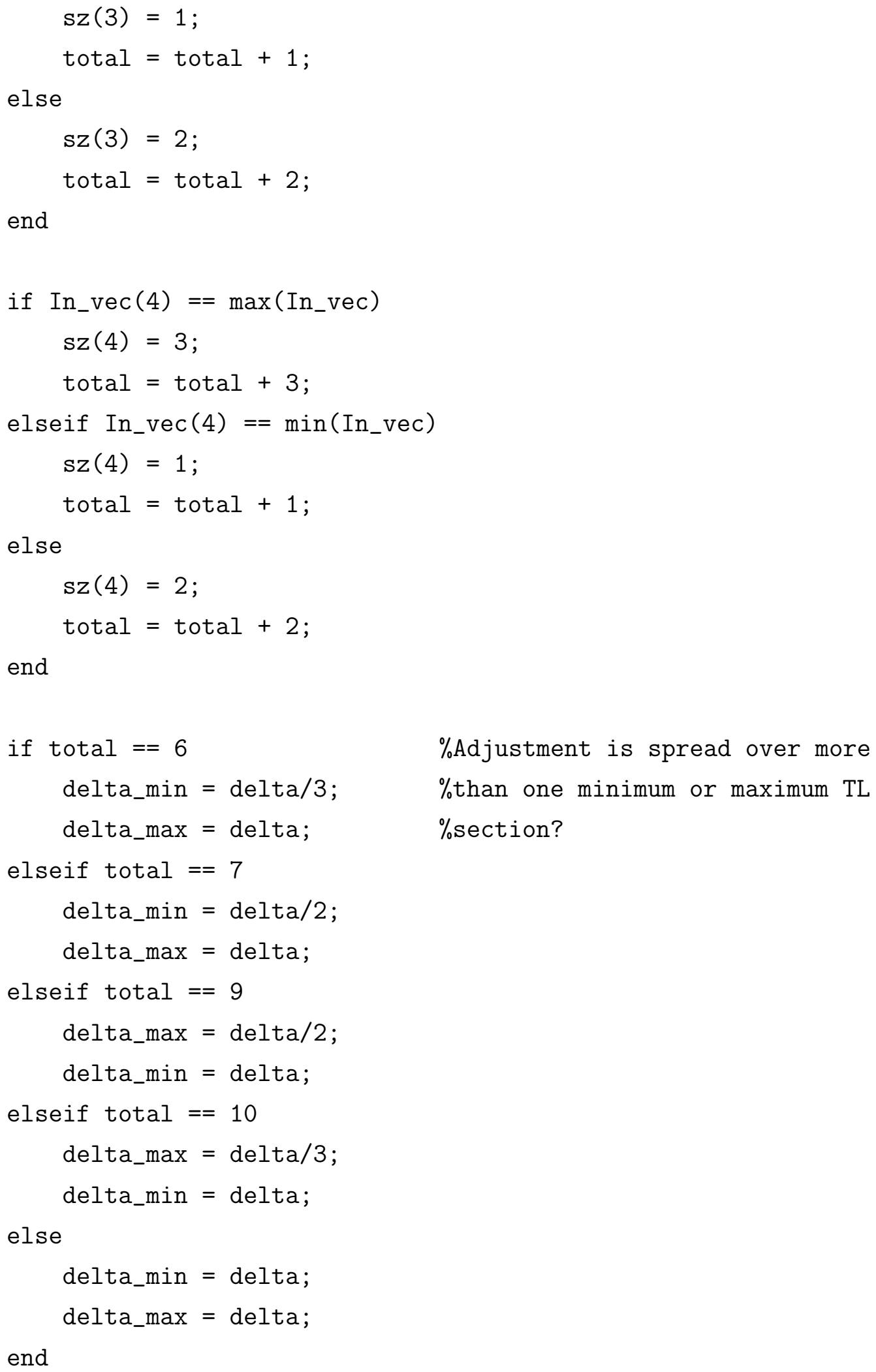




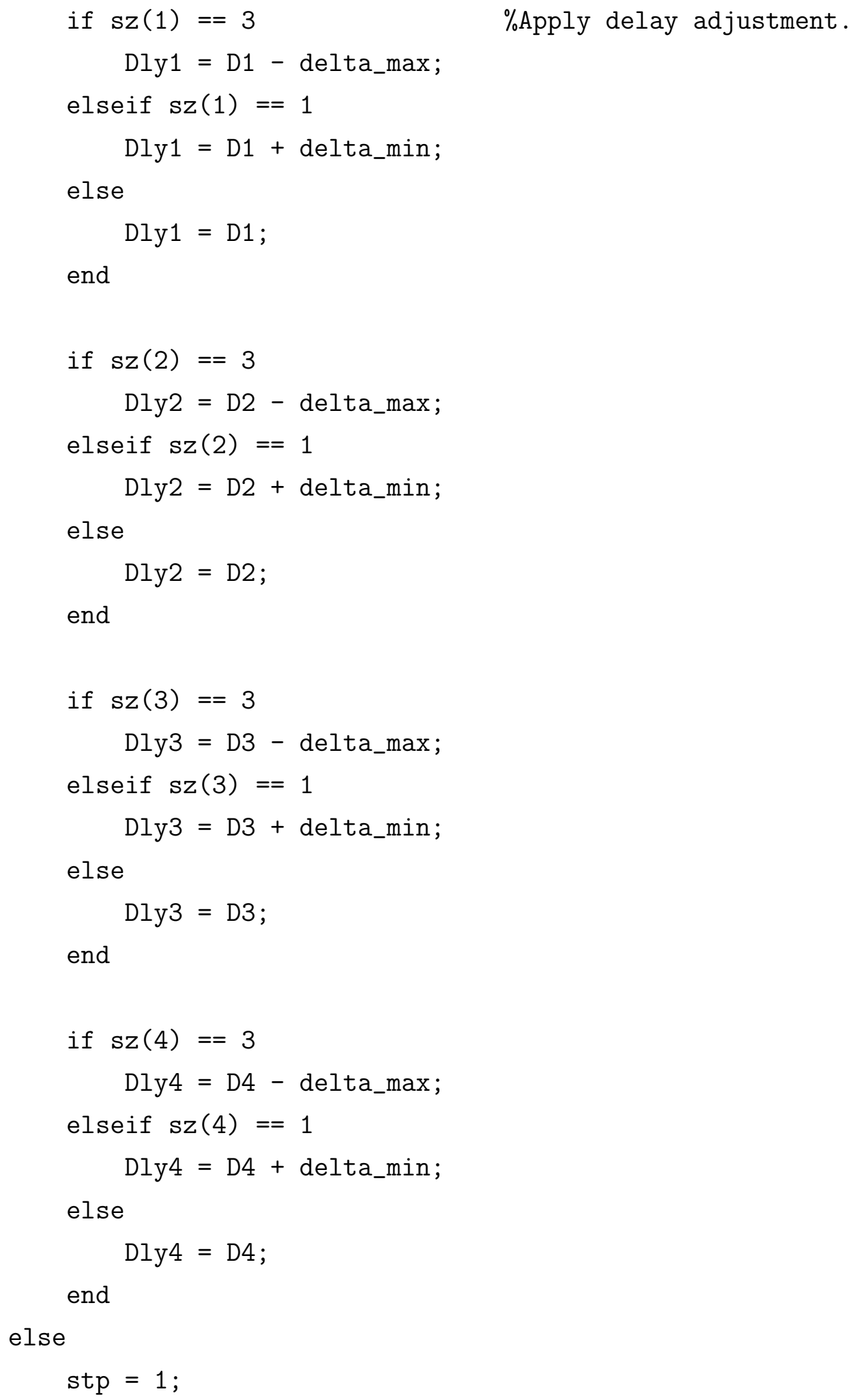




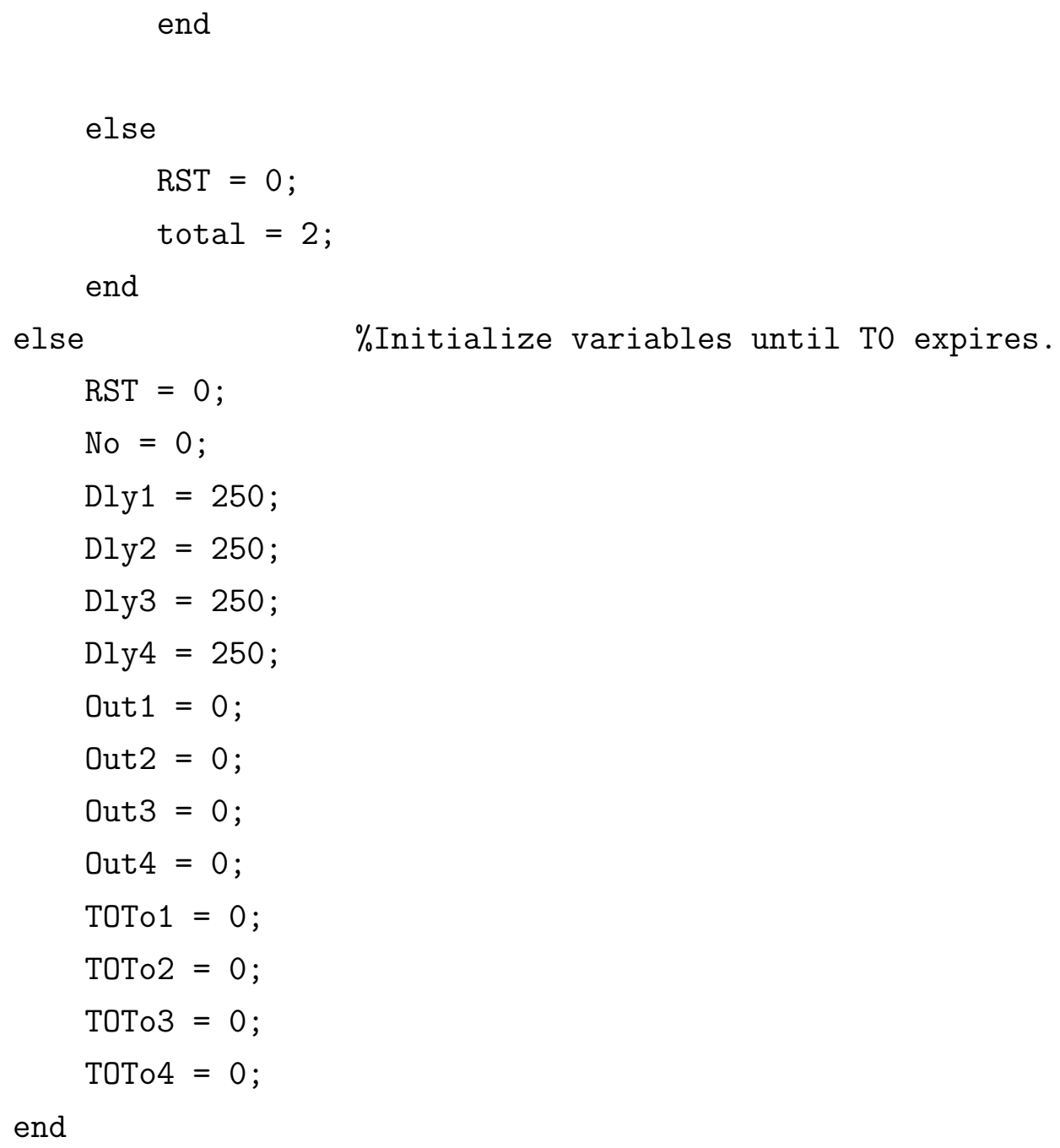




\section{Appendix C}

\section{Test and Evaluation Printed Circuit Boards}

\section{C.1 Phase Detector PCB}

This section describes the PCB that was used to evaluate both versions of the SXOR phase detector. Figure C.1 is the schematic diagram of the phase detector test card. 


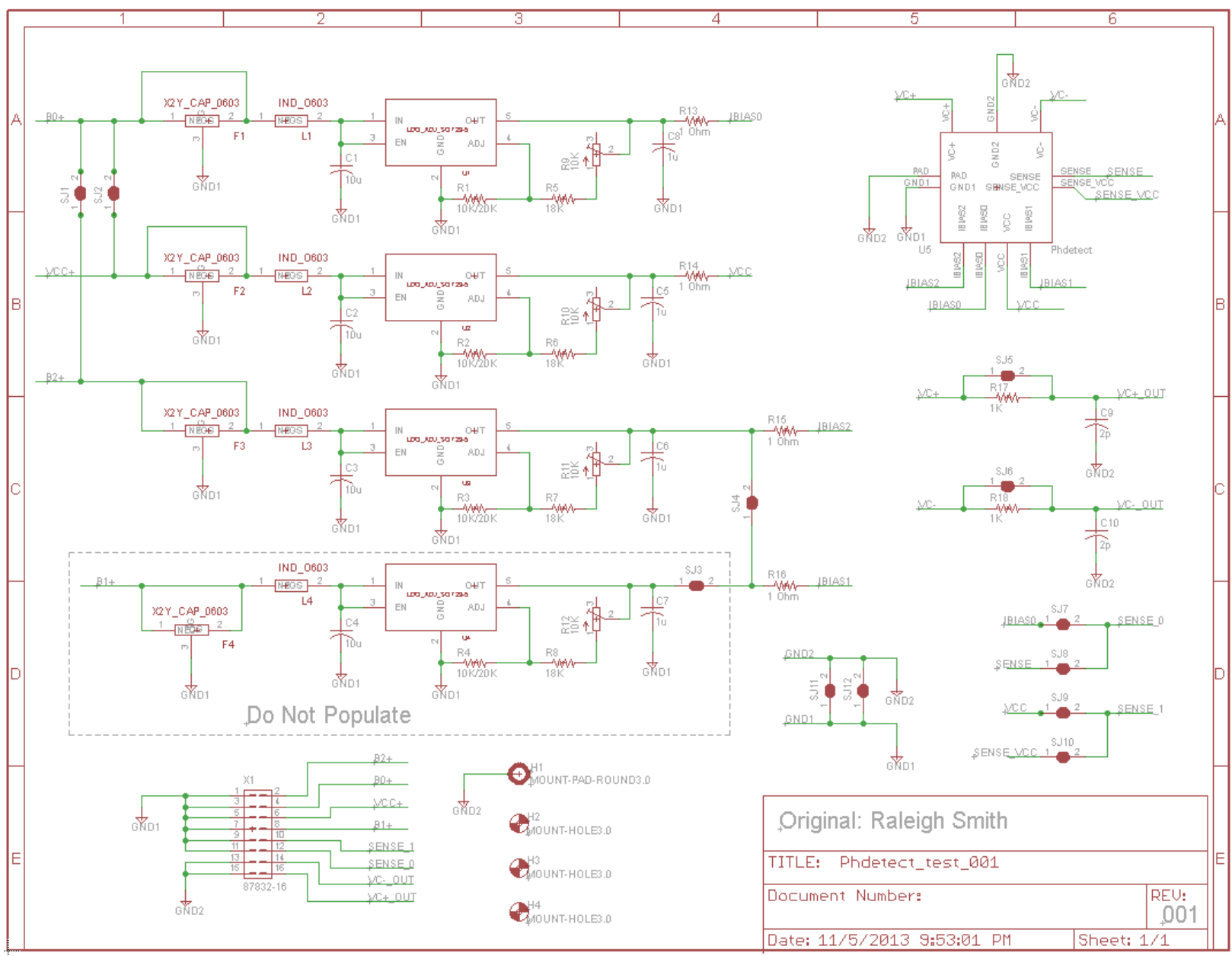

Figure C.1: Phase Detector Test Card Schematic

Figure C.2 is the bill of materials of the phase detector test card. 


\begin{tabular}{|c|c|c|c|c|c|}
\hline Item & Reference & Quantity & Manufacturer & Description & Part Number \\
\hline 1 & PCR1 & 15 & SneedỵPCR.com & Phase Detector Test PCB & Phdetert_nO1 \\
\hline 2 & $\mathrm{C} 5, \mathrm{C} 6, \mathrm{C} 7, \mathrm{C} 8$ & 4 & AVX & $0603, X 7 R, 1 U F, 16 V, 10 \%$ & 0603YC105KAT2A \\
\hline 3 & $R 1, R 2, R 3, R 4$ & 4 & Panasonir & $0603,10 \mathrm{~K} ; 1 \%$ & FRI-3FKF1002V \\
\hline 4 & $\mathrm{R} 1, \mathrm{R} 2, \mathrm{R} 3, \mathrm{R} 4$ & 4 & Panasonic & $0603,20 K, 1 \%$ & ERI-3FKF $2002 \mathrm{~V}$ \\
\hline 5 & $\mathrm{R} 5, \mathrm{R} 6, \mathrm{R} 7, \mathrm{R} 8$ & 4 & Panasonic & $0603,18 \mathrm{~K}, 1 \%$ & FRI-3FKF1802V \\
\hline 6 & $\mathrm{x} 1$ & 1 & Molex & 8783)-16 Circuit Header, Shrouded & 0878371620 \\
\hline 7 & $\mathrm{R} 9, \mathrm{R} 10, \mathrm{R} 11, \mathrm{R} 12$ & 4 & Bourns & 10K, 10 Turn Pot, SMT, Vertical & $3224 X-1-103 E$ \\
\hline 8 & 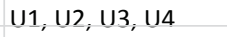 & 4 & ST-Micrnelectronics & SOT23-5I, I ow Noise, Adj, I DO, $1.8-5.5 \mathrm{~V}$ & I DCIO15MR \\
\hline 9 & $\mathrm{C} 1, \mathrm{C}), \mathrm{C} 3, \mathrm{C} 4$ & 4 & $A V X$ & $1206, X_{i}, 10 u F, 10 V$ & 12067(106КАТ)А \\
\hline 10 & $\mathrm{C} 9, \mathrm{C} 10$ & $\underline{2}$ & AVX & $0603, \mathrm{NPO}, 2 \mathrm{pF}, 50 \mathrm{~V}, 0.25 \mathrm{pF}$ & 06035A2ROCAT2A \\
\hline 11 & $\mathrm{R} 17, \mathrm{R} 18$ & ? & Panasonic & $0603,1 \mathrm{~K}, 1 \%$ & FRI-3FKF1001V \\
\hline 12 & R13, R14, R15, R16 & 4 & Panasonic & 0603, 1 Ohm, $1 \%$ & ERJ-3RQF1ROV \\
\hline 13 & $11,12,13,14$ & 4 & Murata Flectronics NA & $0603,43 \mathrm{nH}, 2 \%, 380 \mathrm{~mA}, 2.7 \mathrm{GH} 7$ & I OW18AN43NGOOD \\
\hline 14 & $F 1, F 2, F 3, F 4$ & 4 & Inhanson Dielectrics & $0603, \mathrm{X} \supset \mathrm{Y}, 100 \mathrm{nF}$ & $100 \times 14 \mathrm{~W} 104 \mathrm{MV} 4 \mathrm{~T}$ \\
\hline 15 & & 50 & Molex & Terminals, 24 - 30 AWG & 503948054 \\
\hline 16 & & 4 & Molex & Housing, $2 \mathrm{~mm}, 16$ Pos, Polarized & 511101651 \\
\hline
\end{tabular}

Figure C.2: Phase Detector Test Card BOM

Figure C.3 is the primary side of the fabricated phase detector test card, which shows the component placement including the DUT.

\section{U5 DUT}

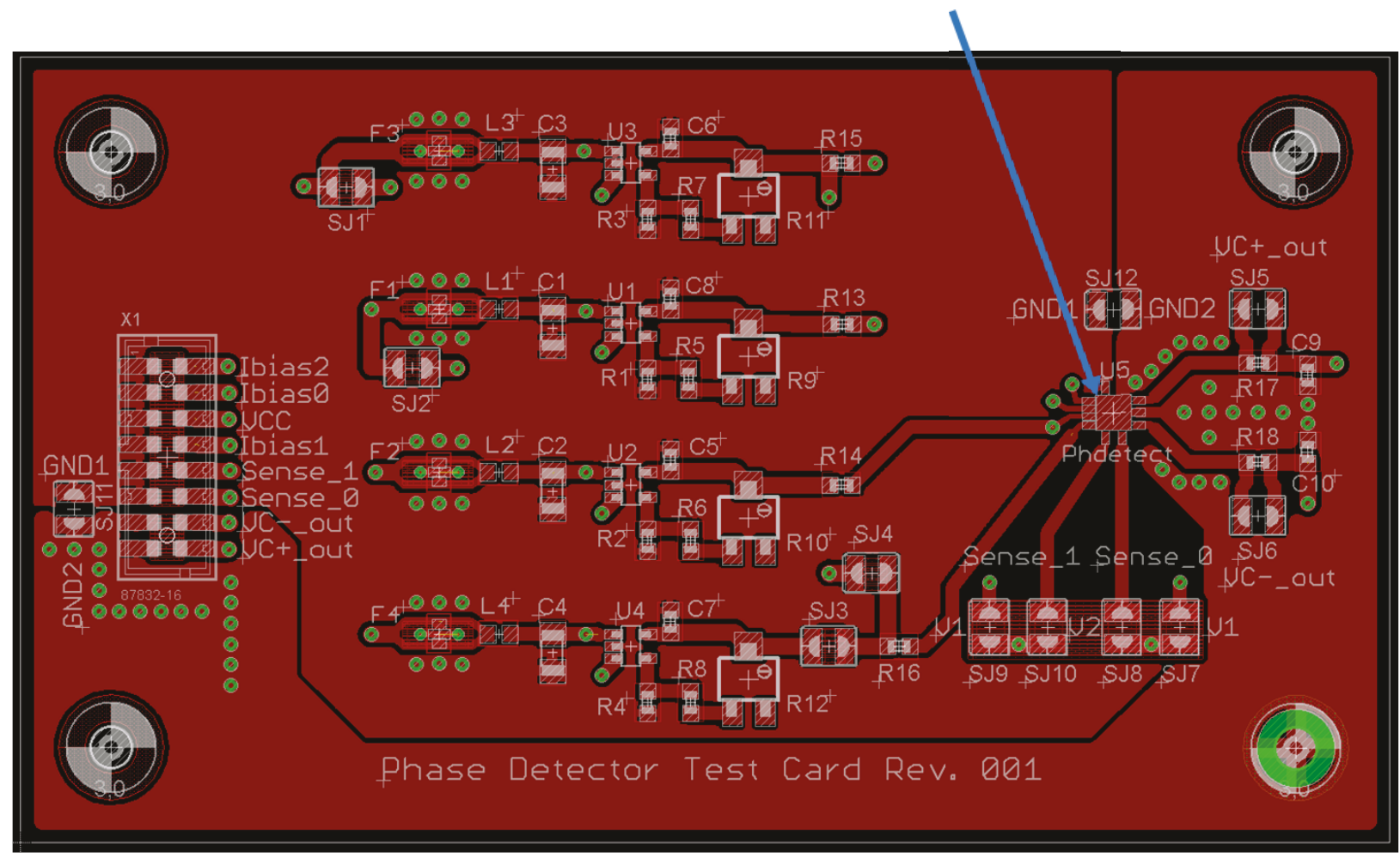

Figure C.3: Phase Detector Test Card Layout - Primary Side

Figure C.4 is the secondary side of the fabricated of the phase detector test card. 


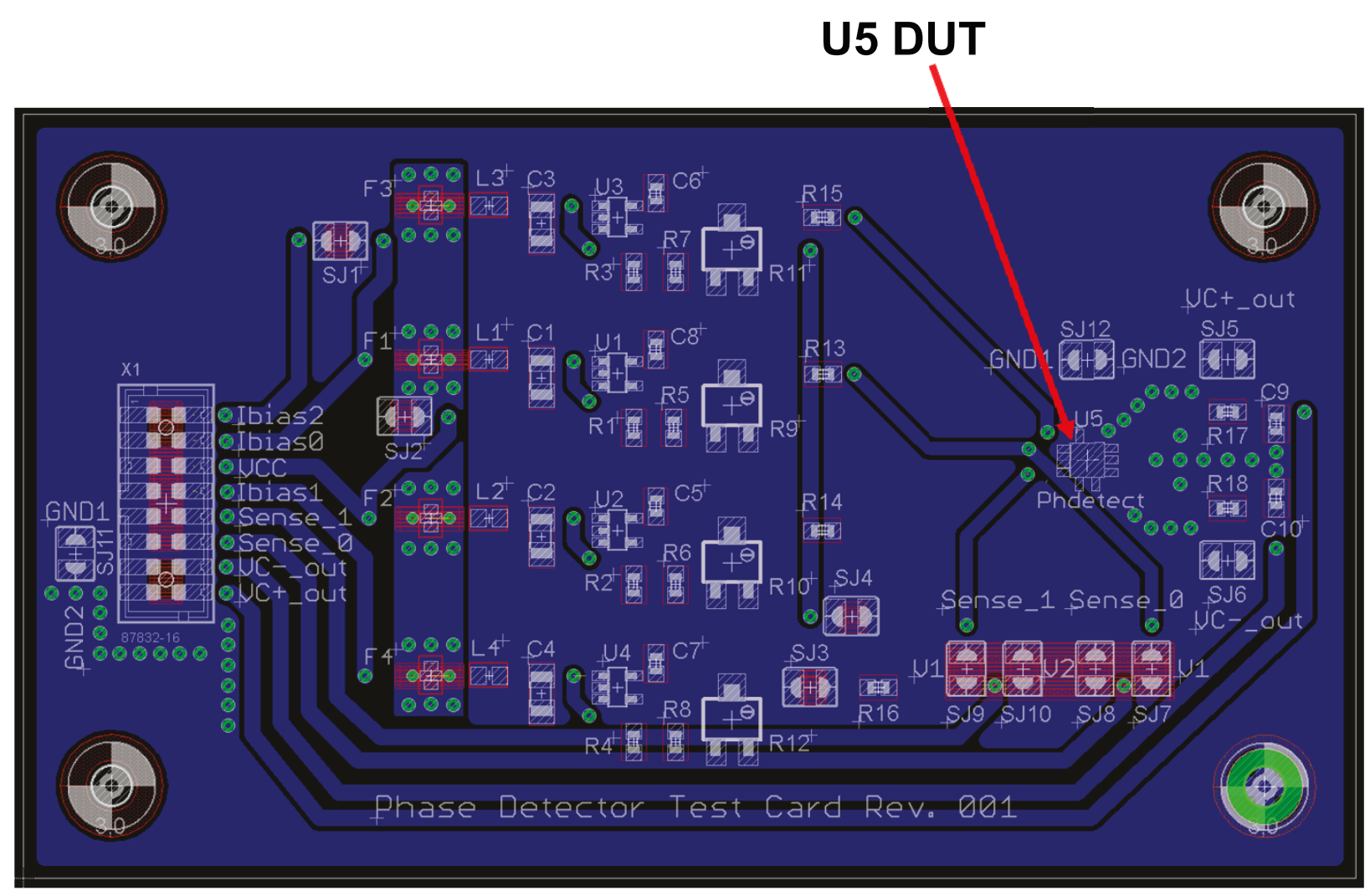

Figure C.4: Phase Detector Test Card Layout - Secondary Side

\section{C.2 RTWO Phase Alignment PCB}

This section describes the PCB that was used to evaluate the tuning of the RTWO provided by Kimia Ansari. Figure C.5 is page one of the schematic diagram of the RTWO tuning test card. 


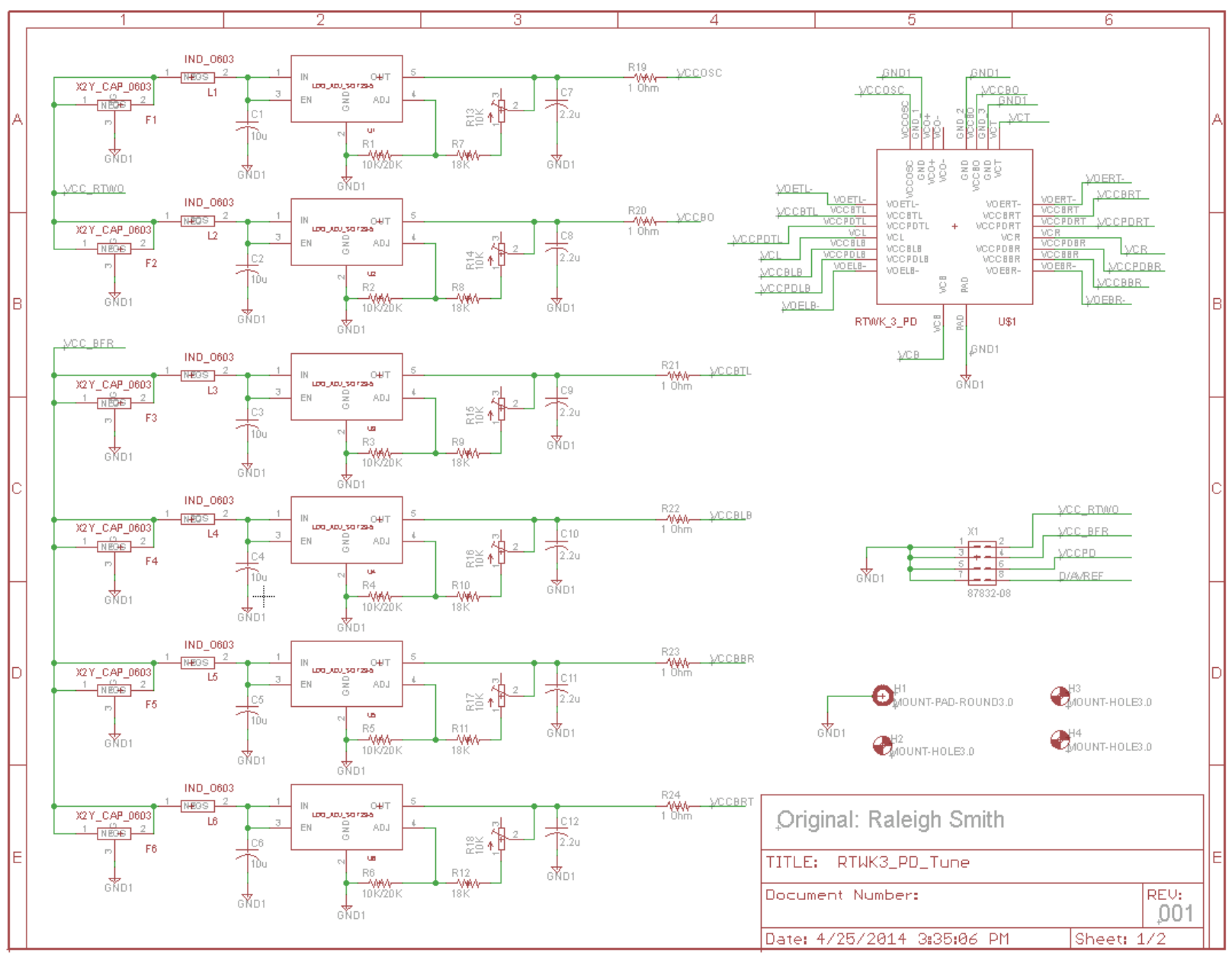

Figure C.5: RTWO Tuning Test Card Schematic - Page 1

Figure C.6 is page two of the schematic diagram of the RTWO tuning test card. 


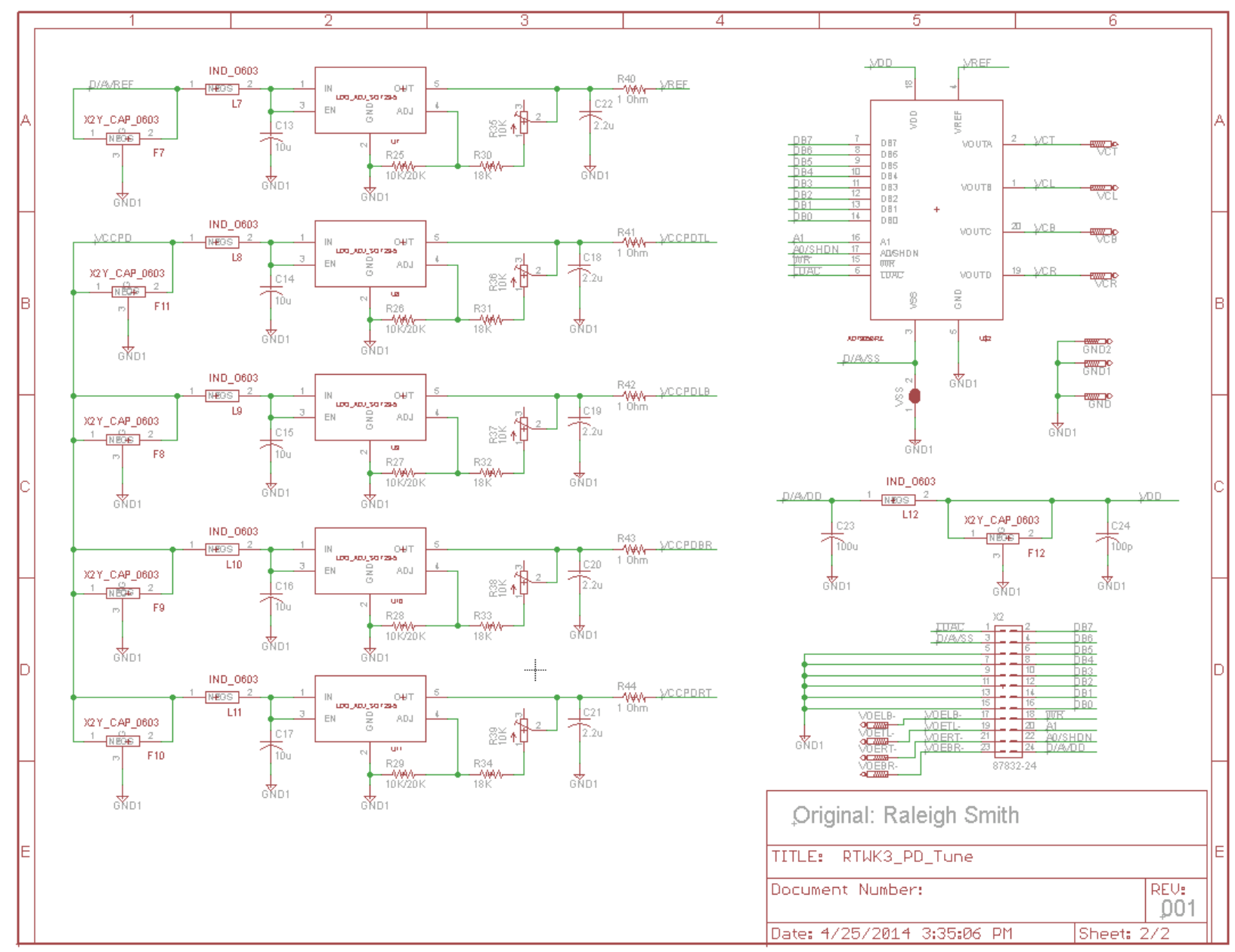

Figure C.6: RTWO Tuning Test Card Schematic - Page 2

Figure C.7 is the bill of materials of the RTWO tuning test card. 


\begin{tabular}{|c|c|c|c|c|c|}
\hline Item & Reference & Quantity & Manufacturer & Description & Part Number \\
\hline 1 & PCB1 & 5 & SpeedyPCB.com & RTWO/Phase Detector Test Card & RTWK3_PD_Tune \\
\hline 2 & $\begin{array}{l}\mathrm{C} 7, \mathrm{C} 8, \mathrm{C} 9, \mathrm{C} 10, \mathrm{C} 11, \mathrm{C} 12, \\
\mathrm{C} 18, \mathrm{C} 19, \mathrm{C} 20, \mathrm{C} 21, \mathrm{C} 22\end{array}$ & 11 & AVX & $0603, \mathrm{X} 5 \mathrm{R}, 2.2 \mathrm{uF}, 10 \mathrm{~V}, 10 \%$ & 0603ZD225KAT2A \\
\hline 3 & $\mathrm{C} 23$ & 1 & $A V X$ & $1210, \times 5 R, 100 u F, 6.3 V, 20 \%$ & 12106D107MAT2A \\
\hline 4 & 024 & 1 & $A V X$ & $0402, x 7 R, 100 n F, 16 \mathrm{~V}, 10 \%$ & O4OगY(101KAT)A \\
\hline 5 & $\begin{array}{l}R 1, R 2, R 3, R 4, R 5, R 6, \\
R>5, R>6, R>7, R>8, R>9\end{array}$ & 11 & Panasonic & $0603,10 K, 1 \%$ & ERJ-3EKF1002V \\
\hline 6 & $\begin{array}{l}R 7, R 8, R 9, R 10, R 11, R 12, \\
R 30, R 31, R 32, R 33, R 34\end{array}$ & 11 & Panasonic & $0603,18 \mathrm{~K}, 1 \%$ & ERJ-3EKF1802V \\
\hline 7 & $\mathrm{x} 1$ & 1 & Molex & 87832-08 Circuit Header, Shrouded & 0878320820 \\
\hline 8 & $x>$ & 1 & Molex & 8783)-24 Circuit Header, Shrouded & 0878322420 \\
\hline 9 & $\begin{array}{l}R 13, R 14, R 15, R 16, R 17, \\
R 18, R 35, R 36, R 37, R 38, \\
R 39\end{array}$ & 11 & Bourns & 10K, 10 Turn Pot, SMT, Vertical & $3224 X-1-103 E$ \\
\hline 10 & $\begin{array}{l}\text { U1, U2, U3, U4, U5, U6, } \\
\text { บ7, บ8, บ9, บ10, U11 }\end{array}$ & 11 & ST-Microelectronics & $\begin{array}{l}\text { SOT23-5L, 300mA, Low Noise, Adj, LDO, } \\
1.8-5.5 \mathrm{~V}\end{array}$ & LDK130M-R \\
\hline 11 & $\begin{array}{l}\mathrm{C} 1, \mathrm{C} 2, \mathrm{C} 3, \mathrm{C} 4, \mathrm{C} 5, \mathrm{C} 6 \\
\mathrm{C} 13, \mathrm{C} 14, \mathrm{C} 15, \mathrm{C} 16, \mathrm{C} 17\end{array}$ & 11 & $A V X$ & $1206, \mathrm{X7R}$, , 10uF, 10V & 1206ZC106KAT2A \\
\hline 12 & $\begin{array}{l}R 19, R 20, R 21, R 22, R 23, \\
R 24, R 40, R 41, R 42 \text { R43, } \\
R 44\end{array}$ & 11 & Panasonic & 0603, 1 Ohm, $1 \%$ & ERJ-3RQF1ROV \\
\hline 13 & $\begin{array}{l}\text { L1, L2, L3, L4, L5, L6, L7, } \\
\text { L8, L9, L10, L11, L12 }\end{array}$ & 12 & Murata Electronics NA & $0603,43 \mathrm{nH}, 2 \%, 380 \mathrm{~mA}, 2.7 \mathrm{GHz}$ & LQW18AN43NGOOD \\
\hline 14 & $\begin{array}{l}F 1, F 2, F 3, F 4, F 5, F 6, F 7, \\
F 8, F 9, F 10, F 11, F 12\end{array}$ & 12 & Johanson Dielectrics & $0603, X 2 Y, 100 n F$ & $100 \times 14 \mathrm{~W} 104 \mathrm{MV} 4 \mathrm{~T}$ \\
\hline 15 & & 150 & Molex & Terminals, 24 - 30 AWG & 503948054 \\
\hline 16 & & 4 & Molex & Housing, $2 \mathrm{~mm}$, 24 Pns, Polarized & $51110-2451$ \\
\hline 16 & & 4 & Molex & Housing, $2 \mathrm{~mm}$, 8 Pos, Polarized & $51110-0851$ \\
\hline
\end{tabular}

Figure C.7: RTWO Tuning Test Card BOM

Figure C.8 is the primary side of the fabricated RTWO tuning test card, which shows the component placement including the DUT. 


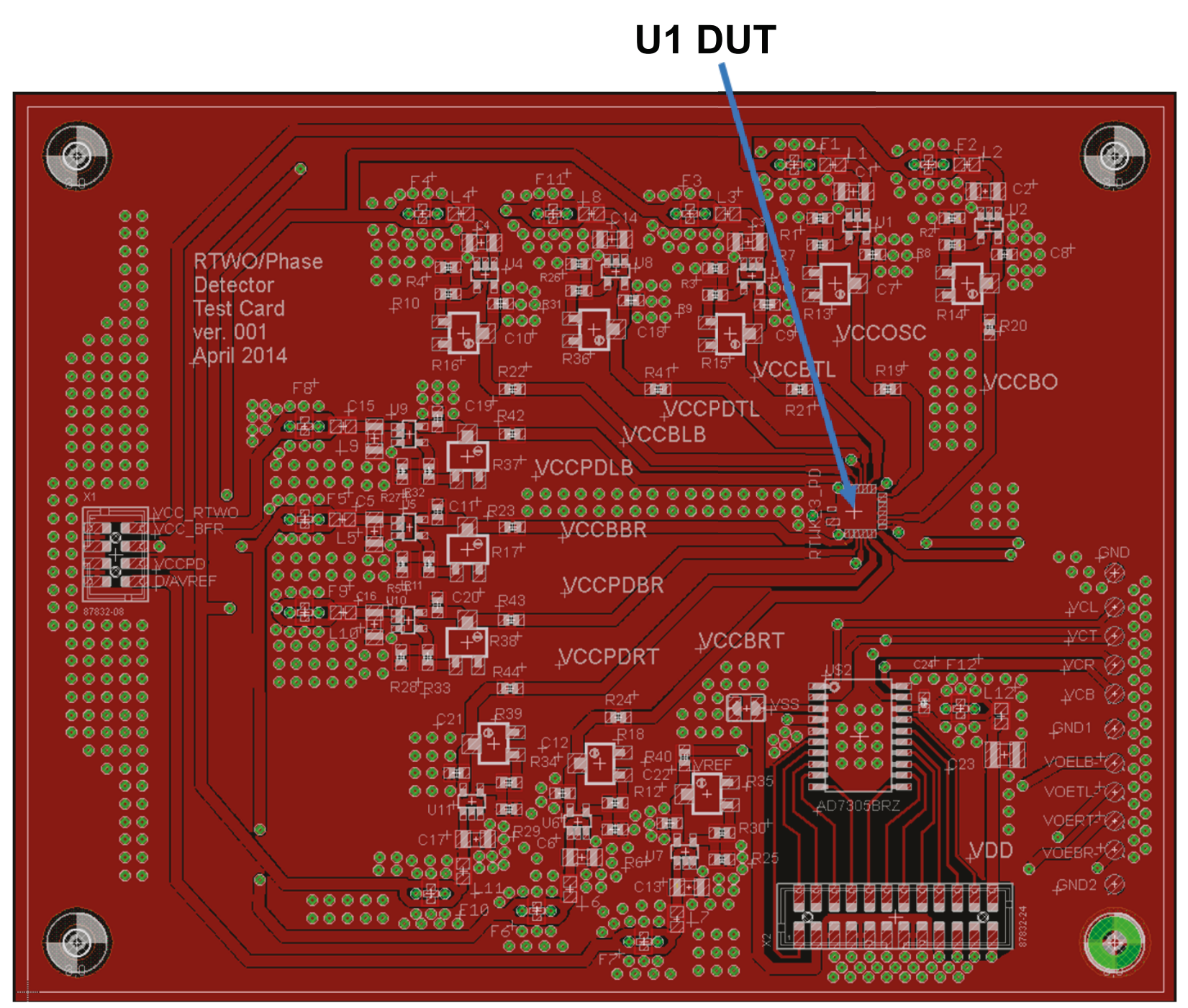

Figure C.8: RTWO Tuning Test Card Layout - Primary Side

Figure C.9 is the secondary side of the fabricated RTWO tuning test card. 


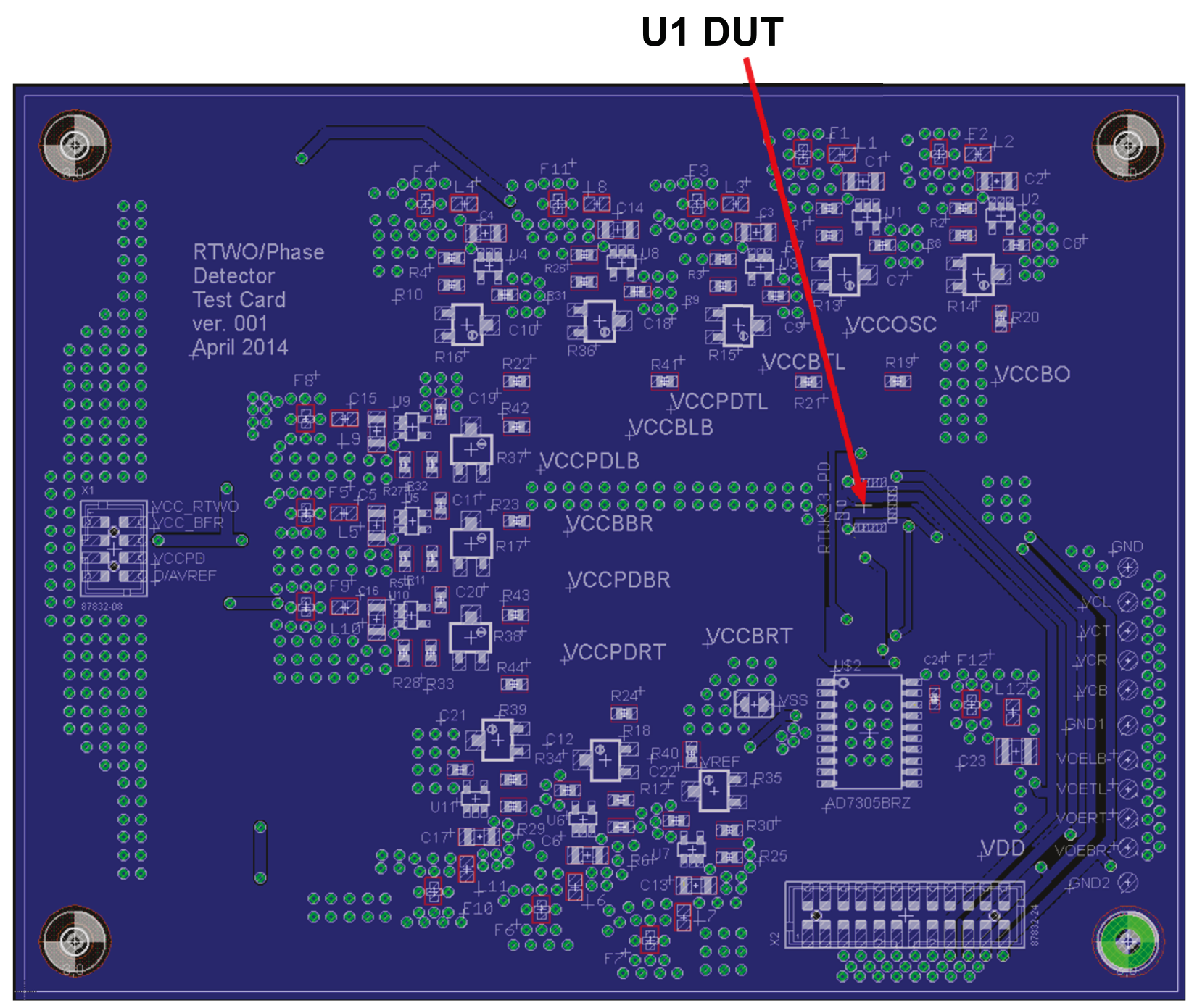

Figure C.9: RTWO Tuning Test Card Layout - Secondary Side 